\title{
Roles of bHLH Transcription Factors Neurod1, Neurod2 and Neurod6 in Cerebral Cortex Development and Commissure Formation
}

\section{INAUGURAL - DISSERTATION \\ zur Erlangung des Doktorgrades der Medizinischen Fakultät der Georg-August-Universität zu Göttingen}

vorgelegt von

Ingo Bormuth

aus

Offenbach am Main 
D e k a n:

I. Berichterstatter:

II. Berichterstatter:

III. Berichterstatter:
Prof. Dr. rer. nat. Heyo Klaus Kroemer

Prof. Dr. med. Mikael Simons

Prof. Dr. rer. nat. Jörg Großhans

Prof. Dr. mult. Thomas Meyer

Tag der mündlichen Prüfung: ７. April 2016 


\section{Contents}

$\begin{array}{ll}\text { Summary } & 1\end{array}$

1 Introduction 3

1.1 Short History of Neurosciences . . . . . . . . . . . . . . . 4

1.2 Cerebral Cortex Development . . . . . . . . . . . . 6

1.2.1 Axis Specification . . . . . . . . . . . . 6

1.2 .2 Arealization ............... 7

1.2.3 Radial Migration . . . . . . . . . . . . . . 9

1.2 .4 Neuronal Identity . . . . . . . . . . . . . . . 11

1.2 .5 Axon Growth . . . . . . . . . . . . 11

1.2 .6 Connectivity . . . . . . . . . . . . 13

1.3 bHLH Transcription Factors . . . . . . . . . . . . . . . . 14

1.3.1 bHLH Domain . . . . . . . . . . . . . . . . 14

1.3 .2 Classification . . . . . . . . . . . 15

1.3 .3 Functions . . . . . . . . . . . . 16

1.3.4 Neuronal bHLH Proteins . . . . . . . . . . . . 17

1.4 The NeuroD-Family . . . . . . . . . . . . . . . . . . . . 18

1.4 .1 Neurod $1 \ldots \ldots \ldots \ldots$

1.4.2 Neurod2 and Neurod6 . . . . . . . . . . . . 19

1.4 .3 Neurod $4 \ldots \ldots \ldots 20$

1.5 Genetic Inactivation Studies . . . . . . . . . . . . . 20

1.5.1 Inactivation of Neurod $1 \ldots \ldots \ldots 21$

1.5.2 Inactivation of Neurod $2 \ldots \ldots \ldots 22$

1.5.3 Inactivation of Neurod6 . . . . . . . . . . . 23

1.6 Functional Redundancy . . . . . . . . . . . . . . . . 24

1.7 The Cre-LoxP System . . . . . . . . . . . . . . . . . 25

2 Results $\quad \mathbf{2 7}$

2.1 The Neurod6-Lineage of Cells . . . . . . . . . . . . . . . 28

2.2 Inactivation of Neurod6 . . . . . . . . . . . . 30

2.3 Inactivation of Neurod2 . . . . . . . . . . . . 31

2.3 .1 Lethality . . . . . . . . . . . . . . 31

2.3.2 Cortical Connectivity . . . . . . . . . . . 32

2.4 Simultaneous Inactivation of Neurod $2 / 6 \ldots \ldots 33$

2.4 .1 Brain Anatomy . . . . . . . . . . . . 34

2.4.2 Cortex Development . . . . . . . . . . 35

2.4.2.1 Overview ............ 36

2.4.2.2 Radial Migration . . . . . . . . . . 37

2.4.2.3 Subplate. . . . . . . . . . . 39

2.4.2.4 Upper Layers . . . . . . . . . . . . . . . . . . 40

2.4.2.5 Deeper Layers . . . . . . . . . . 41 
2.4 .3 Adult Brain . . . . . . . . . . . . . . . 43

2.4.4 Cortical Connectivity . . . . . . . . . . . . . 44

2.4.4.1 Callosal Projections .......... 44

2.4.4.2 Axon Growth . . . . . . . . . . . 45

2.4.4.3 Fasciculation . . . . . . . . . 46

2.4.4.4 Midline Glia . . . . . . . . . . . . 47

2.4.4.5 Midline Crossing . . . . . . . . . . . 49

2.4.4.6 Subcortical Projections ........ 51

2.4 .5 Synaptic Function . . . . . . . . . . . . 52

2.4.6 Arealization of the Neocortex . . . . . . . . . 54

2.4 .7 Upregulation of Neurod 1 . . . . . . . . . . . . . 55

2.5 Simultaneous Inactivation of Neurod $1 / 2 / 6 \ldots \ldots 56$

2.5.1 Breeding . . . . . . . . . . . . . . 57

2.5 .2 Brain Anatomy . . . . . . . . . . . . . 58

2.5 .3 Hippocampus . . . . . . . . . . . . . 59

2.5.3.1 Granule Neuron Differentiation . . . . . 59

2.5.3.2 Pyramidal Neuron Differentiation . . . . . 60

2.5 .4 Neocortex . . . . . . . . . . . . . . . 61

2.5.4.1 Radial Migration and Laminarization . . . 63

2.5.4.2 Determination and Early Differentiation . 64

2.5.4.3 Terminal Differentiation and Identity . . . 65

2.5.5 Developmental Cell Death . . . . . . . . . . . 66

2.5.6 Cortical Connectivity . . . . . . . . . . . 67

2.5.6.1 Intracortical Projections . . . . . . . 67

2.5.6.2 Subcortical Projections . . . . . . . . 68

2.5.6.3 Thalamocortical Handshake . . . . . . 70

2.6 Other Observations . . . . . . . . . . . . . . . . 72

3 Discussion $\quad \mathbf{7 3}$

3.1 Determination . . . . . . . . . . . . . . 74

3.2 Differentiation . . . . . . . . . . . . . 76

3.2.1 Ventricular Zone . . . . . . . . . . . . 76

3.2 .2 Subventricular Zone . . . . . . . . . . . 77

3.2 .3 Intermediate Zone . . . . . . . . . . . . 78

3.2 .4 Cortical Plate . . . . . . . . . . . . 78

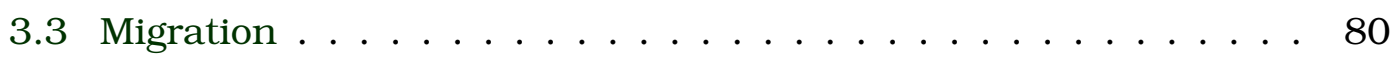

3.4 Arealization ..................... 81

3.5 Axon Growth . . . . . . . . . . . . . . . . . . 83

3.6 Apoptosis . . . . . . . . . . . . . . . . . 85

3.7 Genetic Background . . . . . . . . . . . . . 87

3.8 Adult Functions . . . . . . . . . . . . . . . . . . . . . 87

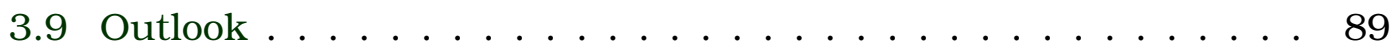

3.9.1 Inducible Neurod6-Cre Mice . . . . . . . . . . . . 89

3.9 .2 Neurod6 Overexpression . . . . . . . . . . . . 90

3.10 Closing Words . . . . . . . . . . . . . . . . 91

4 Material and Methods $\quad 93$

4.1 Transgenic Mice . . . . . . . . . . . . . . . . . . . . . . 93

4.2 Genotyping . . . . . . . . . . . . . . . . 93

4.2 .1 Tissue Lysis . . . . . . . . . . . . . . . . . 94

4.2 .2 Polymerase Chain Reaction . . . . . . . . . . 94 
4.2 .3 Gel Electrophoresis . . . . . . . . . . . . . . 95

4.2.4 Neurod 1-Flox PCR . . . . . . . . . . . . . 95

4.2 .5 Neurod2-Null PCR . . . . . . . . . . . . . 96

4.2 .6 Neurod6-Cre PCR . . . . . . . . . . . . . 97

4.3 Cell Culture . . . . . . . . . . . . . . . . . . 97

4.4 In Utero Electroporation . . . . . . . . . . . . . . . . . . . . . 99

4.5 Electrophysiology . . . . . . . . . . . . . . . . . . . 99

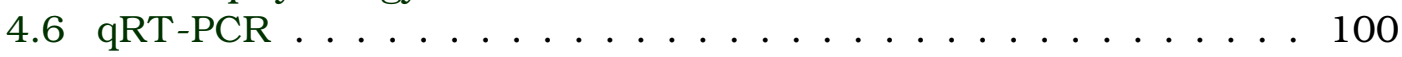

4.7 Histology . . . . . . . . . . . . . . . . . 101

4.7.1 Tissue Preparation and Fixation . . . . . . . . . 101

4.7 .2 Tissue Sectioning . . . . . . . . . . . . . 101

4.7.2.1 Vibratome ............. 101

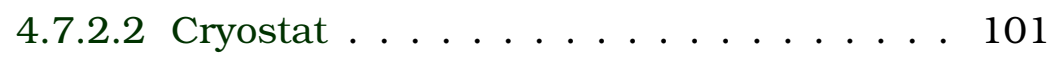

4.7.2.3 Paraffin . . . . . . . . . . . . 102

4.7 .3 X-gal Histochemistry . . . . . . . . . . . . . . . . 102

4.7 .4 Myelin Staining . . . . . . . . . . . . . . . 102

4.7.5 Immunohistochemistry . . . . . . . . . . 103

4.7 .6 In Situ Hybridization . . . . . . . . . . . . . . . 104

4.8 Software . . . . . . . . . . . . . . . . 105

4.8.1 Statistical Analysis . . . . . . . . . . . . . 106

4.8 .2 Sequence Alignment . . . . . . . . . . . . . . . . 107

4.8 .3 Genetic Modeling . . . . . . . . . . . . . . 107

4.8 .4 Image Processing . . . . . . . . . . . . . . 107

4.8 .5 Manuscript Preparation . . . . . . . . . . 108

$\begin{array}{ll}\text { Bibliography } & 109\end{array}$

$\begin{array}{ll}\text { Appendix } & 128\end{array}$

Acknowledgments . . . . . . . . . . . . . . . . . . . . . 129

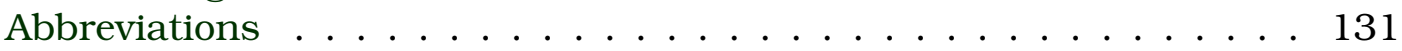

List of Figures . . . . . . . . . . . . . . . . . . . . . . 134

List of Tables . . . . . . . . . . . . . . . . . . . . 135

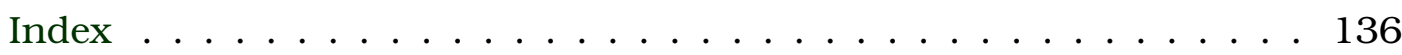

Zusammenfassung (German Summary) . . . . . . . . . . . . . 140 


\section{Summary}

The brain is deeper than the sea,

For, hold them, blue to blue,

The one the other will absorb,

As sponges, buckets do.

E. Dickison ${ }^{1}$

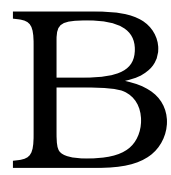

ASIC HELIX-LOOP-HELIX (bHLH) proteins constitute a diverse group of evolutionary well-conserved transcription factors. Many transactivating bHLH proteins follow cell type- or tissue-specific expression patterns and act as key regulators of cellular determination and differentiation processes.

The closely related neuronal bHLH genes Neurod1, Neurod2 and Neurod6 are expressed by differentiating pyramidal neurons in the developing cerebral cortex and have long been suspected to regulate the maturation of these cells. Each of the three genes was genetically inactivated in mice, but studies of single-deficient animals failed to identify important functions in embryonic pyramidal neurons. Considering high sequence similarity and overlapping expression patterns, most authors suggested functional redundancy amongst the NeuroD-family. To test this, I bred transgenic mice lacking the two most similarly expressed NeuroD genes, Neurod2/6; and analyzed cerebral cortex development with an emphasis on pyramidal neuron identity and neocortical connectivity.

Neurod2 and Neurod6 indeed share several hitherto unknown functions and compensate for each other's loss. At least one of the two genes is necessary for: (1) the control of radial migration in a subset of pyramidal neurons; (2) area determination in the neocortex; and (3) the formation of fiber tracts connecting the neocortex to the striatum, to the thalamus, and to the contralateral hemisphere. In Neurod2/6 double-deficient mice, callosal axons form fasciculated fiber bundles that grow tangentially into the medial neocortex, but stall and defasciculate before reaching the ipsilateral cingulum or any midline associated structure. This new variant of callosal agenesis implies the presence of a not yet identified axon guidance mechanism in the medial neocortex.

Neocortical Neurod 1 expression, which is normally restricted to the subventricular zone, persists in the intermediate zone and cortical plate of Neurod2/6 double-deficient embryos. Ectopically upregulated Neurod1 can provide redundant functionality to compensate for the loss of Neurod2/6. I went further and bred conditional Neurod $1 / 2 / 6$ triple-deficient mice, in which the Neurod 1 gene is specifically inactivated in cells with Neurod6-promoter activity.

\footnotetext{
${ }^{1}$ Dickinson 2013, CXXVI, verse 2
} 
As hypothesized, Neurod 1 shares additional functions with Neurod2 and Neurod6. At least one of the three genes is necessary for hippocampal pyramidal neuron differentiation and the prevention of developmental cell death in the medial cortex. While the simultaneous inactivation of Neurod $1 / 2 / 6$ results in the complete loss of archicortical pyramidal neurons, many neocortical pyramidal cells survive, migrate radially and settle in the cortical plate. However, terminal pyramidal neuron differentiation is incomplete and neocortical connectivity is dramatically reduced in the triple-deficient mice.

Taken together, this work shows that NeuroD-family transcription factors cooperatively regulate pyramidal neuron differentiation, survival, migration, specification and axon growth in the developing cerebral cortex.

The partly overlapping functions of Neurod $1 / 2 / 6$ during embryonic cortex development might be summarized as follows: Hippocampal granule cell differentiation and survival depend essentially on Neurod $1.1^{1}$ Early aspects of hippocampal pyramidal neuron differentiation and survival depend on functionality shared by Neurod1, Neurod2 and Neurod6. Later aspects of neocortical pyramidal neuron differentiation, cortical arealization and the guidance of major neocortical axon tracts depend on redundant functionality of Neurod2 and Neurod6, but not Neurod1. Postnatally, the remodeling of the somatosensory cortex in response to functional integration of thalamocortical afferents and the maturation of the hippocampal mossy fiber pathway depend specifically on Neurod2. ${ }^{2}$ Adult functions are largely unknown, although Neurod6 has recently been associated with the development of obsessive-compulsive disorder, schizophrenia and Alzheimer's disease. ${ }^{3}$

\footnotetext{
${ }^{1}$ Miyata et al. 1999; Liu et al. 2000; Schwab et al. 2000

${ }^{2}$ Ince-Dunn et al. 2006; Molnár and Molnár 2006; Wu et al. 2011; Wilke et al. 2012

${ }^{3}$ Mattheisen et al. 2014; Pérez-Santiago et al. 2012; Satoh et al. 2014
} 


\section{Introduction}

\begin{abstract}
The development of the vertebrate nervous system is a problem of such complexity that it is sometimes difficult to know where to start.
\end{abstract}

W. Maxwell Cowan ${ }^{1}$ evolution has created so far. It comprises nearly 100 billion nerve cells ${ }^{2}$ neuronal network contains a large number of specialized subsystems ${ }^{4}$ that operate largely independently and communicate via massive fiber tracts. ${ }^{5}$

The guideline for building, wiring and maintaining the brain is written into every cell's DNA as a highly compressed genetic program ${ }^{6}$. Transcription factors, main subject of this work, change the interpretation of the genetic code in response to cell-intrinsic or cell-extrinsic signals, ${ }^{7}$ thereby providing executional modularity and interactivity.

The last frontier of neuronal sciences is to understand and reproduce the biological processes leading to perception, learning \& memory, reasoning and ultimately consciousness. Many mutations in genes critically involved in embryonic development have been identified to be responsible for cerebral malformations, psychiatric conditions and degenerative disorders. It is becoming increasingly

\footnotetext{
${ }^{1}$ Cowan 1998, p 391, chapter "Lineage analysis in the vertebrate central nervous system"

${ }^{2} \mathrm{~A}$ recent study counted $86 \cdot 10^{9}$ NeuN-positive and $84 \cdot 10^{9}$ NeuN-negative cells in nuclear preparations of male human brains (Azevedo et al. 2009). NeuN is not expressed by CajalRetzius cells, olfactory bulb mitral cells, photoreceptor cells, Purkinje cells, the inferior olivary and the dentate nuclei (Mullen et al. 1992; Sarnat et al. 1998). The total numbers of neurons in the human brain should thus be significantly higher than 86 billion.

${ }^{3}$ The total number of synapses in the brain is very hard to quantify. Synapse densities vary substantially between brain regions, over time, among individuals, and with life style. Assuming an average of 2500 synapses per cell leads to an estimate of 250 trillion synapses per human brain (Bedi et al. 1980 quantified 22270 synapses per neuron in the forebrain and 495 synapses per granule neuron in the cerebellum of young rats; the ratio forebrain/cerebellar tissue weight was $1 / 9$ resulting in a weighted average of 2673 synapses per neuron). The human cerebral cortex alone was estimated to harbor about 150 trillion synapses (Pakkenberg et al. 2003).

${ }^{4}$ He et al. 2009; Meunier et al. 2010; Lo et al. 2011: Network analyses based on human structural and functional MRI data. Estimates for the number of independent functional modules vary between 50 and 4000 .

${ }^{5}$ Thivierge and Marcus 2007: Review on the function of topographic connections in sensory and cognitive networks

${ }^{6}$ Baum 2004, p 52ff: "The goal here is to appreciate the fact that we are nothing but a huge computation [...]"; p 329ff: sect "Gene Expression and the Program of Mind"

${ }^{7}$ Palani and Sarkar 2009: Theoretical model of cellular determination by cross-regulation of transcription factors and cell surface receptors
} 
clear that the key to understanding normal brain function and common disorders such as reduced mental ability, depression, schizophrenia or autism lies in the processes that occur during brain development. ${ }^{1}$

\subsection{Short History of Neurosciences}

$\mathrm{L}$ ATE IN THE 19TH CENTURY, Camillo Golgi discovered the "black reaction", which later became widely known as "Golgi staining". He was the first to depicted individual neurons and their numerous processes in brain sections. ${ }^{2}$ Santiago Felipe Ramón y Cajal consequently applied this silver impregnation technique and could show that the nervous system is not, as previously thought, a continuous network of neuronal fibrils, but instead comprises a very large number of individual nerve cells that are connected by chemical synapses. ${ }^{3}$ During his career as a neuroscientist, Cajal published a vast amount of histological data characterizing many cell- and tissue types in the nervous systems of numerous species. In this vein, he described the cellular structure of the human cerebral cortex $^{4}$ (fig la) and characterized the typical cortical pyramidal neuron. ${ }^{5}$

Subsequently, people have been fascinated with the enormous cellular complexity of the nervous system. Researchers have tried (and still try) to understand how the brain works and by which biological processes it emerges during embryogenesis. During recent decades, a plethora of new scientific methods have been developed and applied to an ever-growing number of questions concerning the perceived 'miracles' of brain function:

The invention of direct immunofluorescence in 1942 made it possible to stain tissue for specific proteins ${ }^{6}$ and to identify cellular and subcellular structures in complex tissue samples (examples in fig 1b-e). In 1951, Eccles and colleagues used fine glass pipettes to record electric potentials from single neurons, and to prove that synaptic transmission is a chemical process. ${ }^{7}$ In 1976 , Neher and Sakman refined this technique to a degree that allowed recording from and characterize single ion channels of living cells. ${ }^{8}$ The deciphering of the genetic code in $1961^{9}$ kick-started the age of molecular genetics. In 1980, the first transgenic mouse was generated ${ }^{10}$ and in 1989 it became possible to alter the

\footnotetext{
${ }^{1}$ Rubenstein 2011: Review on cortex development with a focus on processes involved in the pathogenesis of psychiatric diseases

${ }^{2}$ Golgi 1873: Original paper in Italian language; translated title: On the structure of the gray matter in the brain

${ }^{3}$ Cajal 1904: Original paper in Spanish language; translated title: Structure of the nervous system of men and vertebrates

${ }^{4}$ Elston 2003: Review article quoting and translating passages from Cajal's original work

${ }^{5}$ García-López et al. 2006: Three dimensional reconstruction of a mouse pyramidal neuron based on 32 serial sections originally processed by Santiago Ramón y Cajal

${ }^{6}$ Coons et al. 1942: Use of anthracene coupled fluorescent antibodies to detect a pathogenic antigen (pneumococcus) in fixed mouse tissue

${ }^{7}$ Brock et al. 1952: Intracellular recordings of cat spinal cord motor neurons

${ }^{8}$ Neher and Sakmann 1976: Patch clamp recordings from frog muscle cells

${ }^{9}$ Crick et al. 1961: Introduction of the concepts of codons and reading frames

${ }^{10}$ Gordon et al. 1980: Oocyte injection based generation of transgenic mice
} 


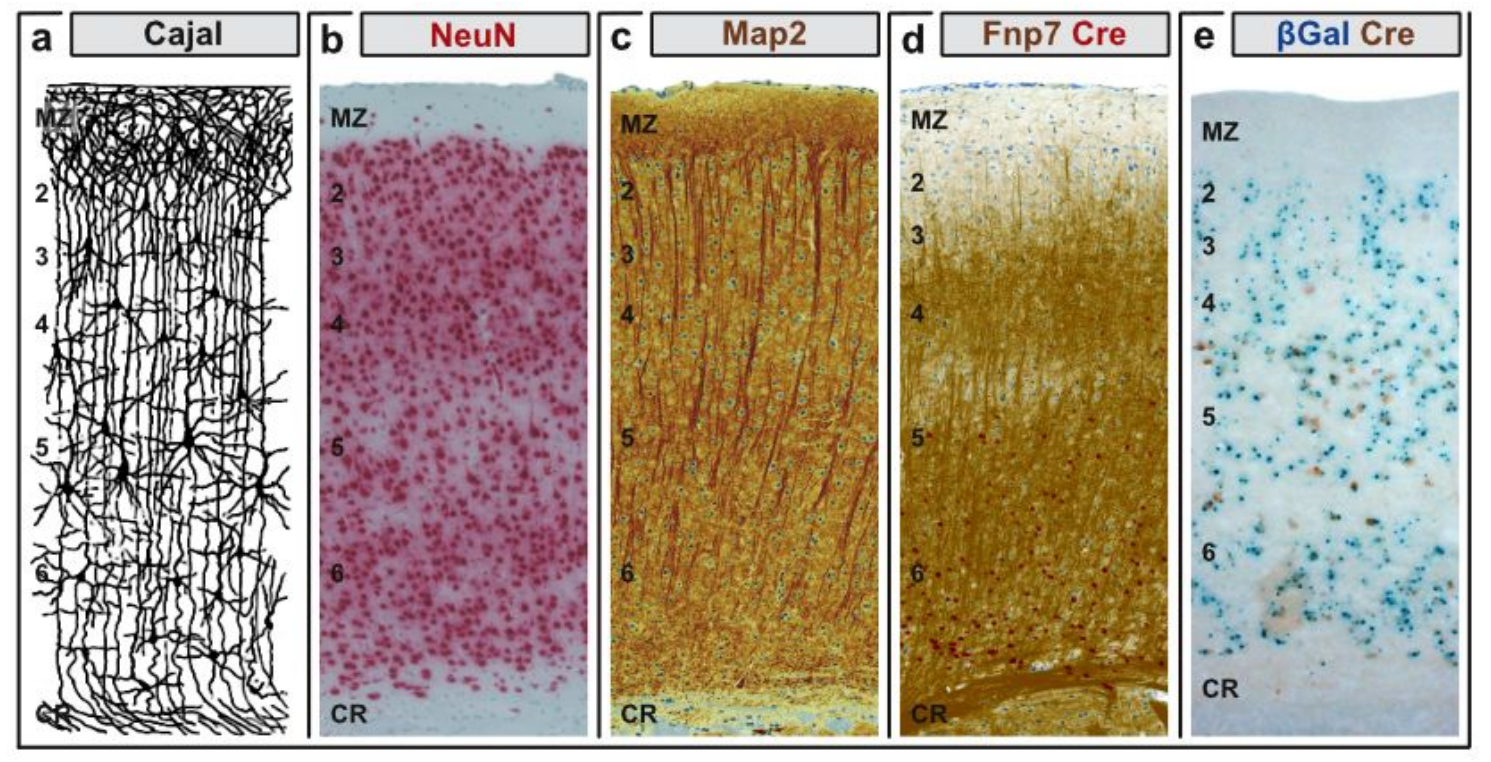

Figure 1: Basic histology of the mouse cerebral cortex

(a) Schematic representation of the six-layered neocortex by Ramón y Cajal. (b-d) Histochemical staining of tissue sections from adult mouse cerebral cortex: (b) NeuN (red) labels the cell bodies of most neurons. (c) Map2 (brown) marks the dendritic compartment. (d) Fnp7 (brown) stains the axonal compartment. Cre recombinase (red) had been introduced into the genome to replace the neuronal transcription factor Neurod6. The expression pattern of Cre resembles that of Neurod6. (e) Histochemical staining reaction (blue) catalyzed by $\beta$-galactosidase. A conditionally expressed variant of the bacterial $\beta$-galactosidase gene (lacZ) had been introduced into the mouse genome. During embryonic development, this construct was permanently activated in all Neurod6 expressing cells by means of Cre mediated genomic recombination at LoxP sites. X-gal precipitates thus label all cells of the Neurod6-lineage (all cells that ever had expressed Cre during their lifetime, and all progeny of those cells). The original drawing reproduced in (a) was first published in Cajal 1904. The copyright expired 2005, 70 years after the author's death in 1934 (EU Directive 2006/116/EC, $1.1,5)$.

sequence of specific genes in mice. ${ }^{1}$ Since 2002 , the genomes of men and mice are fully sequenced ${ }^{2}$ and readily available to the public. ${ }^{3}$ Recently, well-funded initiatives have been launched that aim to map the complete connectivity of the human brain and hope to build computer models that can simulate brain function based upon the exponentially growing amount of experimental data. ${ }^{4}$

In today's neurosciences, it is standard procedure to specifically replace mouse genes by mutated or artificially generated DNA sequences, to analyze the transgenic animal's behavior, visualize the distribution of related proteins and record electrical activity from single neurons. Such studies allow for very detailed views into the overwhelmingly complex biology of the brain.

\footnotetext{
${ }^{1}$ Koller et al. 1989: Gene targeting in mice based on homologous recombination in ES cells

${ }^{2}$ Lander et al. 2001; Venter et al. 2001; Waterston et al. 2002

${ }^{3}$ Genome browser of the Americans National Center for Biotechnology Information (NCBI): http://ncbi.nlm.nih.gov/Genomes/

${ }^{4}$ Kandel et al. 2013: Five popular neuroscientists contemplate on the recently founded American and European brain projects
} 


\subsection{Cerebral Cortex Development}

$\mathrm{T}$ HE ENTIRE CENTRAL NERVOUS SYSTEM (CNS) develops from a specialized ectodermal area stretching along the dorsal aspect of the embryo. ${ }^{1}$ This neuroectodermal tissue is called neuronal plate. The lateral aspects of the neuronal plate thicken and form the interjacent neuronal groove that gradually grows deeper until the upper edges converge and fuse together. The neuroectodermal tissue invaginates to the dorsal aspect of the embryo and forms the neuronal tube. $^{2}$ The caudal part of the neuronal tube is the embryonic precursor of in the spinal cord. The rostral part forms several vesicular protrusions, which give rise to different subdivisions of the brain. Two telencephalic vesicles at the rostral tip of the neural tube eventually form the cerebral hemispheres. ${ }^{3}$ The continuous lumen inside of neural tube and cerebral vesicles persists and develops into the brain's ventricular system.

Neuronal cells are generally born at the inner, ventricular surface and migrate radially into the growing brain. The dorsal part of the telencephalic anlage exclusively produces excitatory neurons. The ventral part generates inhibitory interneurons. Subsets of the ventrally generated interneurons migrate tangentially and integrate into the dorsally situated cerebral cortex. ${ }^{4}$ Dorsally generated pyramidal neurons do not leave the cortex, but instead form very long axonal projections that grow tangentially and connect to distant targets. Targeted neuronal migration and axonal growth depend on molecular signals provided by local environments surrounding cell body and axonal growth cone, respectively. Even at the earliest stages of brain development, spatial information is available to every cell by means of protein gradients along the axes of the CNS anlage. ${ }^{5}$

\subsubsection{Axis Specification}

The neural tube contains two strung-out signaling centers that secrete diffusible molecules forming opposing gradients across its dorsoventral axis. ${ }^{6}$ The ventrally situated floor plate secretes sonic hedgehog (Shh), which instruct the ventral aspect along the neural tube. ${ }^{7}$ The dorsally situated roof plate secretes bone morphogenetic proteins (BMPs) and Wingless and Int homologs (WNTs), which instruct the dorsal aspect ${ }^{8}$ (fig 2a).

\footnotetext{
${ }^{1}$ Kent 1954, p 443ff: Comparison of brain development in different species

${ }^{2}$ Kandel et al. 2000, p 1019, chapter "The Induction and Patterning of the Nervous System"

${ }^{3}$ Bear et al. 1996, pp 12, sect "Understanding CNS structure through development"

${ }^{4}$ Marin 2013, fig 1 "Major groups of cortical interneurons and their developmental origins"

${ }^{5}$ Kandel et al. 2000, pp 1027, sect "The ventral neural tube is patterned by sonic hedgehog secreted from the notochord and floor plate"

${ }^{6}$ Kandel et al. 2000, p 1025, fig 52-5 "Sonic hedgehog and BMP signaling pattern the neural tube along its dorsoventral axis"

${ }^{7}$ Dodd et al. 1998, fig 1: Shh expression in the chick neuronal tube

${ }^{8}$ Chizhikov and Millen 2005, p 291, sect "Roof plate-dependent patterning of the vertebrate dorsal CNS"
} 
a

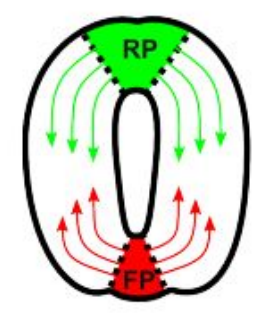

b

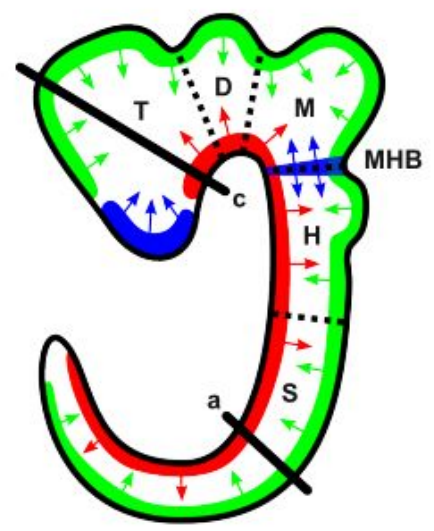

c

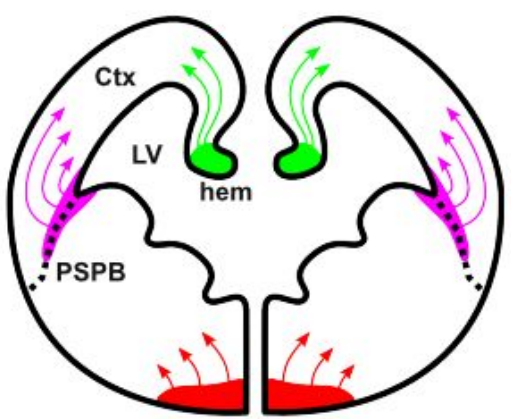

Figure 2: Axis specification in neuronal tube and cerebral cortex

(a) Ventral/dorsal signaling in cross section of the early neuronal tube: The ventral floor plate (FP) secretes Shh (red); the dorsal roof plate (RP) secretes BMP and WNT (green). (b) Overview of the entire CNS anlage: Both ventral/dorsal signaling centers stretch longitudinally from the spinal cord to the telencephalon. Fgf8 secretion (blue) from the rostral tip of the telencephalic vesicle allows for rostral/caudal orientation. An additional Fgf8 secreting center is situated at the midbrain-hindbrain boundary (MHB). (c) Cross section of the developing telencephalon: The dorsal WNT and BMP secreting center is called cortical hem, it patterns the hippocampus and medial neocortex. An additional signaling center at the lateral boundary between dorsal and ventral telencephalon (the pallium/subpallium boundary, PSPB) is called antihem, it secretes Fgf7 and EGFs (eg TGF- $\alpha$ ) and patterns the lateral neocortex. Abbreviations: Ctx, cortex; D, diencephalon; $\mathrm{H}$, hindbrain; LV, lateral ventricle; M, mesencephalon; S, spinal cord; T, telencephalon.

A third signaling center is located at the rostral tip of the neuronal tube. This area secretes predominantly fibroblast growth factor 8 (Fgf8). It defines the rostrocaudal axis of cortex and striatum ${ }^{1}$ (fig $2 b$ ).

Medial-lateral patterning of the telencephalic anlage is defined by the counterplay of hem and antihem, two centers involved in WNT/BMP- and EGF-signaling, respectively. The hem is a longitudinal structure situated at the midline, ${ }^{2}$ it is a descendant of the roof plate that had been folded in ventrally. The antihem is located in the ventrolateral neocortex and is also called the pallium/subpallium boundary (PSPB) ${ }^{3}$ (fig 2c).

\subsubsection{Arealization}

The telencephalon consists of the dorsally located cerebral cortex and the ventrally located ganglionic eminence, the future striatum (fig 2c). As already mentioned, these two structures evolved as functional counterplayers that produce glutamatergic projection neurons and GABAergic interneurons, respectively (sect 1.2). The dissociation of ventral and dorsal identities happens early in development as a direct consequence of dorsoventral axis specification by Shh, BMPs and WNTs. ${ }^{4}$

\footnotetext{
${ }^{1}$ Hébert and Fishell 2008, fig 3: Schematic overview of telencephalic development in Fgf8 of Fgfr deficient mice

${ }^{2}$ Grove et al. 1998, fig 1: WNT genes are expressed in the cortical hem.

${ }^{3}$ Assimacopoulos et al. 2003, fig 2: Genes of the EGF-family are expressed in the cortical antihem.

${ }^{4}$ Ohkubo et al. 2002; Takahashi and Liu 2006; Aboitiz and Montiel 2007
} 
The cerebral cortex comprises the rostrally situated olfactory bulb (paleocortex), the caudally situated hippocampus (archicortex), and the interjacent six-layered neocortex. Olfactory bulb and rostral neocortex are mainly instructed by Fgf8; hippocampus and caudal neocortex are instructed by BMPs and WNTs. These molecules form extracellular protein gradients that translate to smoothly graded expression patterns of intracellular transcription factors along the cortical surface: Pax6 and Sp8 follow rostral to caudal gradients; Emx2 and Nr2f1 (formerly COUP$\mathrm{TF} 1$ ) follow caudal to rostral gradients ${ }^{1}$ (fig 3a, b).

The neocortex is further divided into structurally and functionally distinct areas. Examples for large and well defined areas are the primary somatosensory, visual, auditory, and motor cortex (fig 3c). Borders between cortical areas are usually sharp and often defined by the expression of characteristic genes (eg, Cadherin 8, EphrinA5 and Ror $-\beta$ in the somatosensory cortex). Recently, a large number of very diverse transcriptional enhancer sequences were identified to drive gene expression in sharply defined areas of the developing cortex. ${ }^{2}$ Transcriptional contrast enhancement (the translation of graded to abrupt expression pattern) often relies on direct or indirect self-regulatory feedback loops. ${ }^{3}$ A simplified, hypothetical model of transcriptional self-regulation is depicted in fig $3 d-f$. Such a mechanism can robustly produce sharp expression boundaries from smooth multidimensional protein gradients. However, all efforts to identify strictly areaspecifically expressed transcription factors in the developing cerebral cortex have been futile so far. ${ }^{4}$ The molecular mechanisms defining the positions and boundaries of cortical sub-systems must thus be more complex. Area specific gene expression is probably defined by combinations of many transcription factors that are expressed in characteristic but overlapping pattern along the cortical surface. In such a scenario, numerous transcription factors would act in direct cooperation or competition to drive or inhibit the expression of the same target gene. The resulting expression pattern would depend on all involved transcription factors, the impact of a single one could be relatively small. Transcription factors would qualify as modulators of dynamic expression pattern, rather than simple on/off switches. The inactivation of single factors would not abolish, but rather shift or blur the expression domain of the target gene. This is exactly what happens in the cerebral cortex: Loss of Nr2f1 or Emx2, for example, results in caudally shifted arealization. ${ }^{5}$ The inactivation of Pax6 leads to an oppositely orientated, rostral shift.

${ }^{1}$ O'Leary et al. 2007a: Regulation of Pax6, Sp8, Emx2 and Nr2f1 by Fgf8 and WNTs (fig 6); Borello and Pierani 2010.

${ }^{2}$ Visel et al. 2013 used p300 based ChIP-Seq to identify 4600 genomic DNA sequences predicted to be transcriptionally active during forebrain development. They generated transgenic reporter mice for 329 of these candidates and monitored reporter activity during embryonic cortex development (fig 1,2). Pattabiraman et al. 2014 is a continuation of that work describing the activity of selected enhancers in more detail and showing that these sequences are bound by the Nr2f1, Pax6 and Pbx1, transcription factors known to regulate cortical patterning.

${ }^{3}$ Transcriptional feedback loops define eg the MHB (Picker et al. 2002, fig 7,8) and code segmentation of the spinal cord (Rubenstein and Rakic 2013, sect 7.5.1, fig 7.7).

${ }^{4}$ Rubenstein and Rakic 2013, p 75, sect 4.6.1: “Therefore, area-specific genes per se either do not exist or are exceedingly rare [...] Instead, a neocortical area is defined by the expression of a unique subset of genes, each of which is also expressed in other areas."

${ }^{5}$ Armentano et al. 2007: In conditional Nr2f1 mice, somatosensory and visual cortex were reduced in size and shifted to the caudal pallium; the motor cortex was enlarged and occupied nearly the entire pallium. 
a

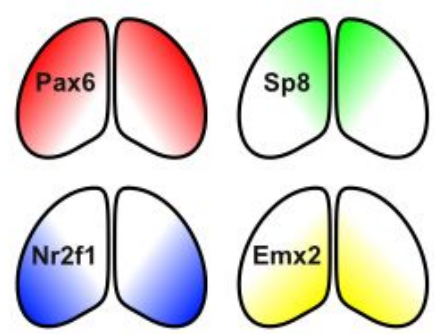

d

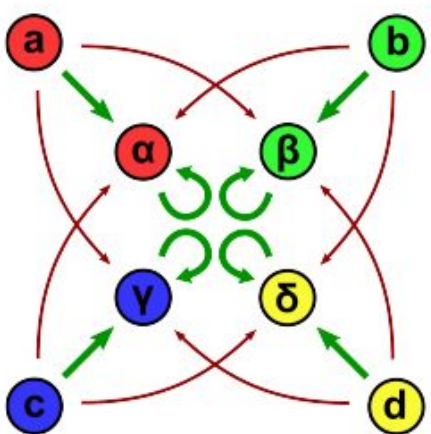

b

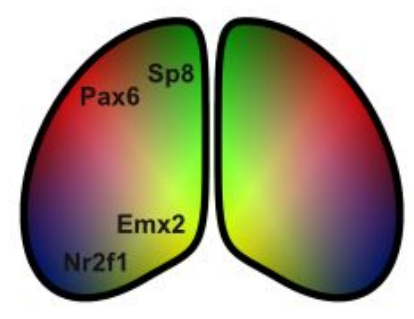

e

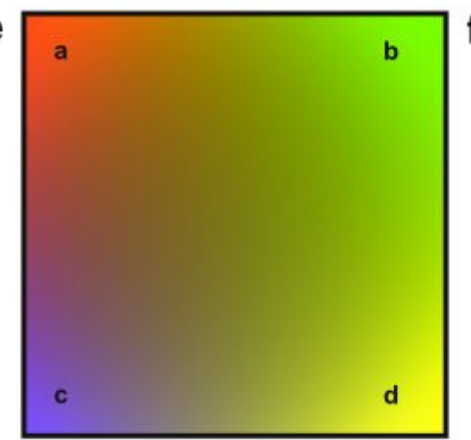

c
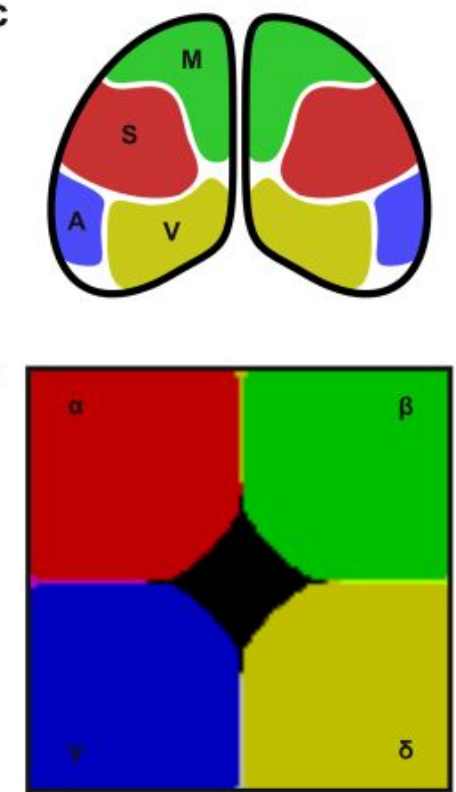

Figure 3: Transcriptional control of neocortical arealization

(a) Extracellular gradients of diffusible signaling molecules (eg, Fgf8, WNT/BMP, Shh) translate to graded expression pattern of intracellular transcription factors along the cortical surface: Among many others, Sp8 (green) and Nr2f1 (blue) define the rostromedial to caudolateral axes; Pax6 (red) and Emx2 (yellow) define the rostrolateral to caudomedial axis. (b) Additive overlay of the Sp8, Nr2f1, Pax6 and Emx2 expression pattern. (c) The main areas of the neocortex (M, motor cortex; S, somatosensory cortex; V, visual cortex; A, auditory cortex). The boundaries between these areas are usually sharp. Areas are defined by histological features and sets of specifically expressed genes. Reproduction based on Borello and Pierani 2010. (d) Simplified model (sect 4.8.3) describing the translation of graded to abrupt expression patterns based on positive feedback loops. The four hypothetical genes $a-d$ follow graded expression pattern and induce sharp expression domains of the transcription factors $\alpha-\delta$. Green and red arrows depict activating and inhibitory transcriptional activity, respectively. (e) Additive overlay visualizing the graded expression pattern of the genes $a-d$. (f) Additive overlay visualizing the sharply defined and mutual exclusive expression pattern of the genes $\alpha-\delta$. Interestingly, such area specifically expressed transcription factors are not known in the developing cerebral cortex, and the control of cortical arealization is probably much more complex.

In summary, cells are able to sense local concentrations of extracellular signaling molecules and translate those to the synthesis or modification of proteins. Transcription factors directly changes the cell's interpretation of the genetic code, thereby providing executional interactivity of the genetic program. Permanent restrictions of the programmatically reachable state space correspond to steps in cellular differentiation and ultimately lead to the establishment of cellular identity. The synchronization of cellular identities results in the formation of homogeneous tissues or tissue areas.

\subsubsection{Radial Migration}

The neuroepithelium is the innermost cell layer of the telencephalic anlage. It ensheaths the lateral ventricle and is therefore called the ventricular zone (VZ). The VZ is mitotically very active and harbors neuronal stem cells, which are also termed radial glia cells, because they feature a long cytoplasmic process that extends radially towards the brain surface. Stem cells undergo symmetric (vertical) or asymmetric (horizontal) mitosis. Symmetric cell divisions produce 
two stem cells and thereby increase the ventricular surface area. Asymmetric divisions result in one stem cell to maintain the stem cell pool, and one neuronal progenitor cell that quickly migrates radially to leave the VZ. During early cortex development, before E14, most neuronal progenitors differentiate directly into neurons. At later stages, most undergo an intermediate stage in the subventricular zone (SVZ), where they undergo several rounds of mitosis before continuing radial migration and neuronal differentiation. ${ }^{1}$ Those two neurogenic cell types are called apical and basal (intermediate) progenitors, respectively.

Committed neurons settle temporarily in the intermediate zone (IZ) where they undergo basic neuronal differentiation and initiate axon growth. Having acquired the typical bipolar morphology of young pyramidal neurons, they continue to migrate radially towards the brain surface. Within the developing cortical plate (CP), later born cells travel in-between already settled pyramidal neurons. The cortex is thus built inside-out: deeper (lower) layers are generated first; outer (upper) layers are born last. ${ }^{2}$

Cell bodies of radially migrating pyramidal neurons follow the courses of their apical dendrites, which have been anchored in the marginal zone (MZ) at the outer surface the brain. The MZ comprises Cajal-Retzius cells, the earliest born neurons of the cerebral cortex. ${ }^{3}$ Cajal-Retzius cells typically express the cadherin associated protein Ctnnb1, the semaphorin Sema6a and the secreted extracellular matrix protein Reelin. They promote radial migration into the $\mathrm{CP}$, and they are thought to ultimately present a stop signal to prevent pyramidal neurons from invading the $\mathrm{MZ}$.

The standard nomenclature of neocortical layering is unfortunately purely descriptive: Layer 1 corresponds to the most superficial MZ, it is devoid of pyramidal neurons and thus not part of the CP. Layer 2 is the most superficial subset of pyramidal cells, which are born late during cortex development $(\sim \mathrm{E} 16)$. Layer 6 is the deepest layer of pyramidal cells, which are born early during cortex development ( $\sim$ E12). Layer 3-5 reside between layer 2 and layer 6. Layer 5 contains the evolutionary oldest and visually very prominent motoneurons (Betz cells in humans). Layer 7 is a temporal structure that defines the inner border of the CP, it comprises the very heterogeneous population of subplate (SP) cells, most of which are born before the emergence of layer 2 .

\footnotetext{
${ }^{1}$ Noctor et al. 2004: Symmetric and asymmetric divisions of radial glia cells in the VZ and neuron production by intermediate progenitors in the SVZ were directly visualized by confocal time laps imaging of oganotypic slice cultures from in utero electroporated rat brains.

${ }^{2}$ Angevine and Sidman 1961: Serial injections of radioactively labeled thymidine into pregnant mice at E11, E13, E15 and E17 showed the sequential but overlapping generation of deeper and at later stages upper cortical layers.

${ }^{3}$ Bielle et al. 2005 used genetically modified mice expressing $\beta$-galactosidase, Cre recombinase or diphtheria toxin A from the endogenous Dbxl-promoter to trace origins and migration patterns of Cajal-Retzius cells in the cortical MZ. The first Dbx1- and Reelin-positive cells were detected at E10.5-E1 1, 1-2 days before the formation of the CP. Together with Takiguchi-Hayashi et al. 2004, this data shows that Cajal-Retzius cells constitute a non-uniform population of neurons that migrate tangentially into the early cortical MZ and originate from independent sources, such as the cortical hem, the PSPB or the septum.
} 


\subsubsection{Neuronal Identity}

Cortical pyramidal neurons share an eponymous triangular morphology that features a large apical dendrite, multiple smaller basal dendrites, and an axon that projects to distant targets and forms excitatory (glutamatergic) synapses. Nevertheless, different subtypes exist that are characterized by substantially different molecular identities, sub-cellular morphologies, axonal targets and electrophysiological properties. Pairs of pyramidal neurons that were born at the same time but in distant cortical areas tend to share more similarities than others that were born in close proximity but at different phases of cortex development. A cortical layer thus represent a relatively homogeneous subpopulation of similar neurons, while a cortical area represents a structurally heterogeneous functionally module.

Some stage-specifically or layer-specifically expressed genes serve as molecular markers for different pyramidal neuron identities. ${ }^{1}$ Examples of marker genes used during this study include Pax6 for neuronal stem cells in the VZ; Eomes (Tbr2) for basal progenitors in the SVZ; NeuN for determined neurons in the SVZ, IZ and CP; Ctgf for the subplate (layer 7); Sox5 and Foxp2 for layer 6; Ctip2 for the deeper layers 5/6; Cux1 for the upper layers 2/3; Satb2 for callosally projecting pyramidal neurons in layer $2 / 3 / 5$, and Reelin for Cajal-Retzius cells and the MZ (layer 1).

\subsubsection{Axon Growth}

Pyramidal neuron precursors in the SVZ/IZ are multipolar. They feature immature neurites that extend and retract randomly, but that do not yet qualify as axons or dendrites. Eventually, one neurite undergoes axonal specialization, while all others become dendrites. The selection of the axonal process is called neuronal polarization. The most critical underlying mechanisms is mutual inhibition: Every neurite inhibits axonal specification of its neighbors. Larger (more axonal) neurites cause stronger inhibition. After an initial very dynamic phase, one single process acquires the capability to inhibit axonal specification of all others; this process ultimately qualifies as the axon. Neuronal polarization and the selection of the axonal process can additionally be influenced by extracellular signals. ${ }^{2}$

The tip of a growing axon is called the growth cone. This highly specialized structure navigates axonal elongation, based on environmental cues. Dynamic micro-processes (filopodia) continuously probe the surrounding tissue for molecular guidance cues. The growth cone repeatedly moves itself into the direction of those filopodia that integrated the highest ratio of attractive vs. repulsive signals. Continuous directed relocation of the axonal tip ultimately leads to targeted axon growth ${ }^{3}$ over very long distances. ${ }^{4}$ To reach their specified target, outgrowing

\footnotetext{
${ }^{1}$ Molyneaux et al. 2007, fig 3 provides an overview 66 different layer specifically expressed marker genes.

${ }^{2}$ Arimura and Kaibuchi 2007 sect "Extracellular signals and polarity"; Sakakibara and Hatanaka 2015, sect "Extracellular mechanisms"

${ }^{3}$ Dent and Gertler 2003, fig 2 "Stages of Axon and Branch Growth"

${ }^{4}$ Smith 2009: Spinal axons of blue whales can reach a maximum length of about 30 meters. The elongation of spinal projection axons is achieved by passive stretching that is taken to the
} 
axons must follow complex paths through different tissue environments that present a variety of relevant and irrelevant guiding cues. It is important to realize that the axonal path is coded into the dynamic receptor configuration of the growth cone. The following is an incomplete list of some important axon guidance cues and receptors:

- Many molecules involved in the determination of early tissue axes (BMPs, WNTs, Shh, FGFs) can later act as axon guidance signals. ${ }^{1}$

- Netrins are diffusible signaling molecules that can attract (via Dcc receptors) or repel (via Unc5 receptors) axonal growth. Netrin 1 is secreted from the cortical midline and the ganglionic eminence. Dcc and Unc5 are expressed by cortical pyramidal neurons. The timing and relative ratio of cellular Dcc and Unc5C expression guides axons of neocortical pyramidal neurons to follow medially or laterally oriented trajectories, respectively. ${ }^{2}$

- Ephrins comprise a large group of guidance molecules that can bind to Eph receptors. Interestingly, ephrin signaling can be bidirectional: Many ephrin ligands also function as receptors and transduce intracellular signals after binding to Eph receptors, which then act as functional ligands. ${ }^{3}$

- Semaphorins comprise a large family of repellent molecules. Compatible receptors are Plexins, Integrins and Neuropilins. Neuropilin 1 signaling is involved in the guidance of early pyramidal neuron axons away from the brain surface towards the SP. ${ }^{4}$

- Slits are diffusible guidance molecules that bind to Robo proteins. During cortex development, Robo1/2 and Slit1 are expressed by cortical pyramidal neurons while Slit 1-3 and Robo3 are expressed by cell populations located at the midline. Robo-Slit signaling it important for midline crossing of commissural axons. Genetic inactivation of Slit2, Slit3 or Robo1, but not of Slit1 or Robo2 leads to defects in interhemispheric cortical connectivity. ${ }^{5}$

- Neurotransmitter receptors located in the growth cone can guide axonal growth in response to neuronal activity. ${ }^{6}$

Many guidance cures and receptors are very dynamically expressed during embryogenesis. Targeted axon guidance depends on very precise timing of very different developmental events.

extreme in big whales: “...blue whale spinal axons growing at $3 \mathrm{~cm} /$ day represent an increase in volume that is likely more than double the volume of the entire neuron cell body-each day."

${ }^{1}$ Charron and Tessier-Lavigne 2005, tab 1: Overview of commissural phenotypes following the inactivation of Shh, BMP and WNT genes in several species.

${ }^{2}$ Srivatsa et al. 2014: Ctip2 and Satb2 determine subcerebral and callosal projections, respectively. Satb2 represses Ctip2 expression leading to mutual exclusive expression pattern in most cortical pyramidal neurons. Ctip2 and Satb2 repress Unc5c and Dcc, respectively. The unbalanced ratio of Ctip2/Satb2 expression thereby controls the ratio of Unc5c/Dcc expression, which influences the lateral versus medial guidance choice of efferent cortical axons.

${ }^{3}$ North et al. 2013: Ephrin signaling is essential in all stages of cortical development and function. This review discusses the control of mitosis, neuronal migration, axon guidance, cortical arealization, synapse formation, synaptic plasticity and apoptosis.

${ }^{4}$ Strittmatter 2000; Polleux et al. 2000

${ }^{5}$ Andrews et al. 2006; López-Bendito et al. 2007; Unni et al. 2012

${ }^{6}$ Erskine and McCaig 1995; Kreibich et al. 2004; Ruediger and Bolz 2007 


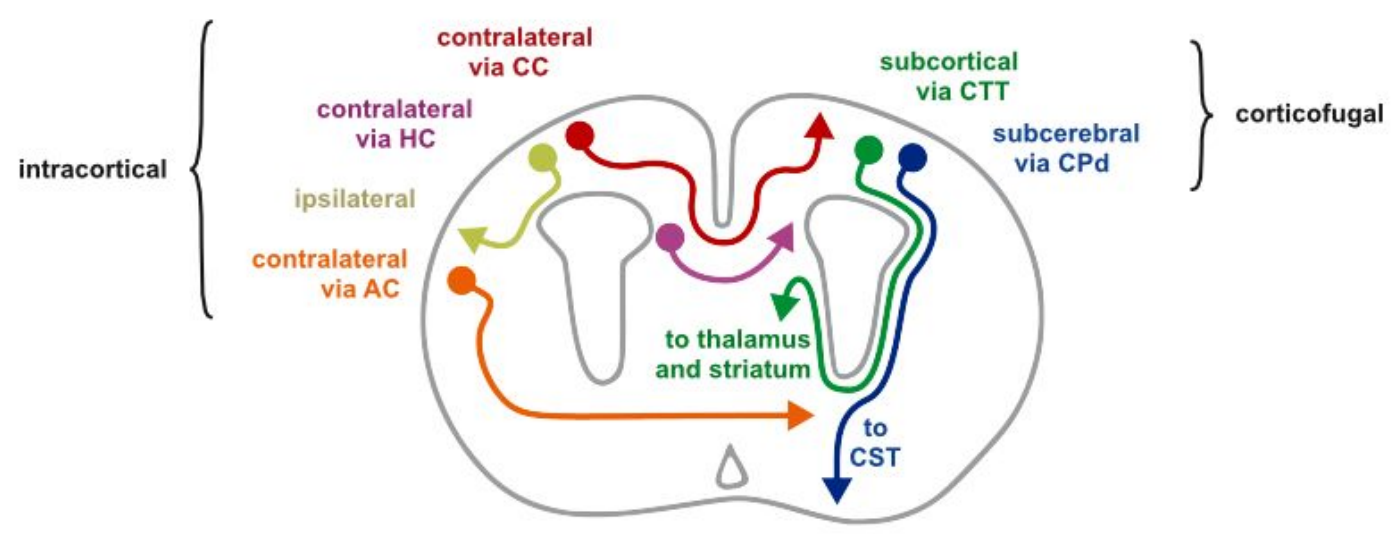

Figure 4: Major neuronal projection tracts of the cerebral cortex

\subsubsection{Connectivity}

Neocortical pyramidal neurons can be classified by means of their primary axonal targets (fig 4): Intracortical projections connect to other cortical areas; corticofugal projections send their axons away from the cortex and connect to subcortical structures such as the thalamus, the striatum, the brain stem or the spinal cord. ${ }^{1}$

Intracortical projection neurons target other pyramidal neurons in the ipsilateral or contralateral hemisphere. Ipsilateral projections originate in layer 2/3 and connect to local or remote cortical areas of the same hemisphere, often forming hierarchical association networks. ${ }^{2}$ Commissural projection neurons interconnect the two cortical hemispheres. They originate in layer 2-6 and send their axons through the corpus callosum (CC) and (to a lesser extent) through the anterior commissure (AC). ${ }^{3}$ Commissural axons mostly target pyramidal neurons located in identical cortical areas of the contralateral hemisphere. They synchronize the two hemispheres and allow for the lateralization of certain functions (eg, the processing of speech) to only one side of the brain. ${ }^{4}$

Corticofugal projections travel through the internal capsule to leave the cerebral cortex and target other parts of the CNS. They can further be classified into subcortical projections that originate from layer 6 and target the thalamus, and subcerebral projections that originate from layer 5 and connect to neurons in the pons, the tectum and the spinal cord. ${ }^{5}$

\footnotetext{
${ }^{1}$ Koester and O'Leary 1993: Tracing of callosal and subcortical fiber projections by injection of different dyes into rat brains

${ }^{2}$ Herron and Johnson 1987: Tracing of intracortical fiber projections by HRP injections into the raccoon somatosensory cortex

${ }^{3}$ Weller et al. 1987: Tracing of callosal and ipsilateral fiber projections by HRP injection into the tree shrew cortex

${ }^{4}$ Kevanishvili et al. 1969: EEG recordings during and after callosotomy in cat and dog

${ }^{5}$ Kaneko et al. 2000, fig 3: Retrograde tracing of corticospinal and corticothalamic projections in the rat cerebral cortex
} 

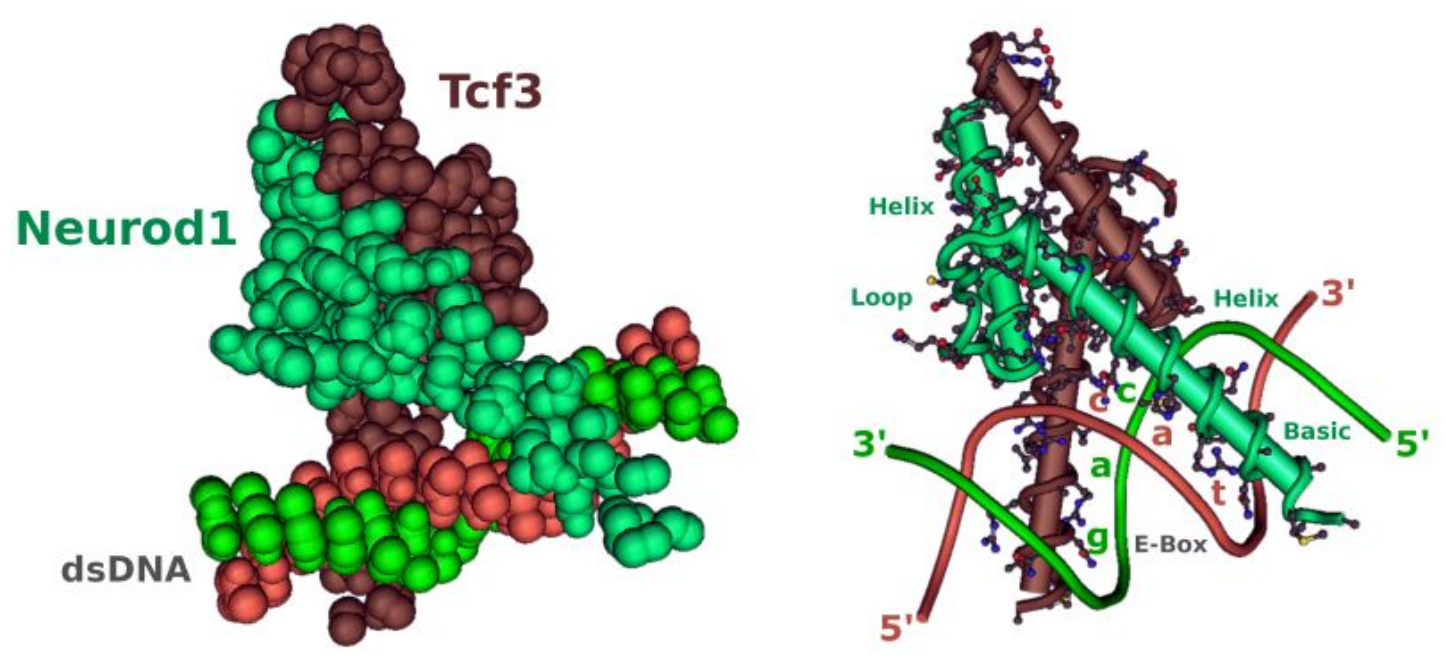

Figure 5: Structure and heterodimerization of bHLH proteins

Depicted is a heterodimer of Neurod1 (cyan) and Tcf3 (brown) binding the palindromic E-box sequence CATCTG of a short DNA fragment (orange/green). Neurod1 binds the sense strand and Tcf3 the antisense stand. Only bHLH domains are shown. The figure was produced based upon published X-ray crystal structure data from Longo et al. 2008.

Subsets of cortical pyramidal neurons form axon collaterals and simultaneously project to different targets. For example, some callosal projection neurons connect to the contralateral cortex, the contralateral striatum, and the ipsilateral striatum at the same time. ${ }^{1}$

\section{3 bHLH Transcription Factors}

$\mathrm{T}$ HE DIVERSE FAMILY of basic helix-loop-helix (bHLH) transcription factors comprises more than 130 related and evolutionary well conserved proteins in humans. ${ }^{2}$ The number of bHLH genes usually increases with the complexity of an organism. This can be explained by genetic duplications and diversification during phylogenesis. Examples for this tendency are the backer's yeast (Saccharomyces cerevisiae), the worm (Caenorhabditis elegans), the fly (Drosophila melanogaster), the house mouse (Mus musculus) and humans (Homo sapiens), with around 8, 39, 58, 104 and 125 known bHLH genes, respectively. ${ }^{3}$

\subsection{1 bHLH Domain}

The eponymous bHLH domain spans 60-100 amino acids and comprises two highly conserved $\alpha$-helices, that are linked by a short variable sequence (loop).

\footnotetext{
${ }^{1}$ Sohur et al. 2014, fig 5: Simultaneous retrograde tracing of callosal and corticostriatal projections in the mouse brain

${ }^{2}$ Skinner et al. 2010: Phylogenetic analysis of known bHLH genes from humans, mice, rats, worms, flies, yeast and the plant Arabidopsis

${ }^{3}$ Ledent et al. 2002; Skinner et al. 2010
} 


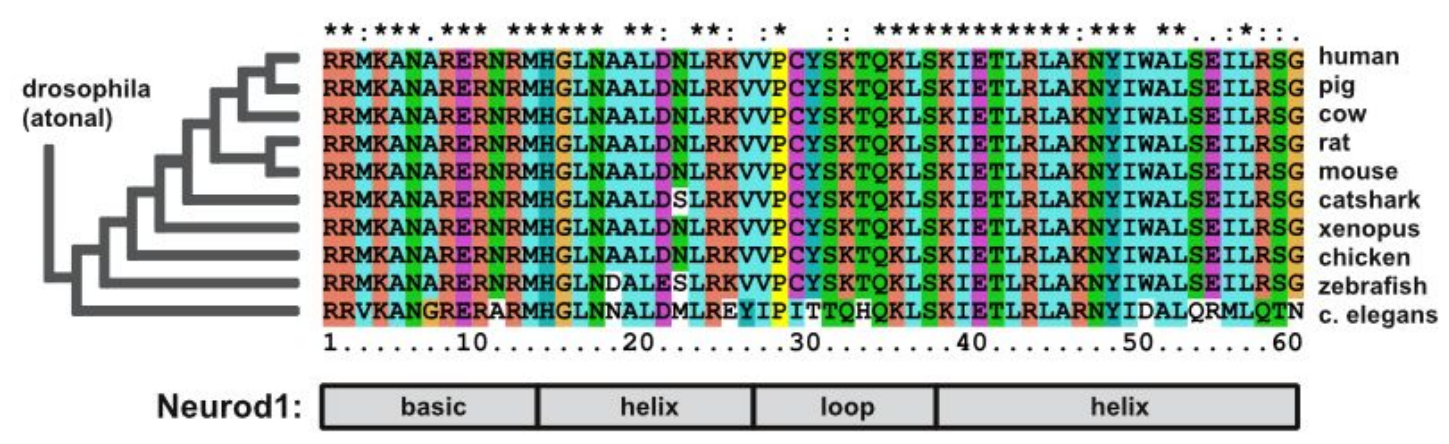

Figure 6: Evolutionary conservation of the Neurod1 bHLH domain

Multiple amino acid sequence alignment comparing the Neurod1 bHLH domains of several species. The phylogenetic tree at the left is based the on complete protein sequences. The bHLH domain is $100 \%$ identical in mouse, rat, cow, pig and men; the basic domain is even identical between zebrafish and men.

Typically, dissimilar bHLH proteins heterodimerize (fig 5), thereby forming a functional (DNA binding) unit. In comparison to many other gene families, bHLH transcription factors have been very well conserved during evolution. The amino acid sequence of the bHLH-domain often remained identical over millions of years (fig 6).

Directly 5' of the helix-loop-helix domain follows a stretch of about 15 basic (positively charged) amino acids. ${ }^{1}$ If two bHLH proteins dimerize, the basic regions face each other and can cooperatively bind short deoxyribonucleic acid (DNA) motives with the consensus recognition sequence CANNTG, the enhancer box (E-box) motive. ${ }^{2}$ To function as active transcription factors, bHLH proteins must carry transactivator domains. However, these are not eminently conserved (even not between related proteins with almost identical bHLH domain).

\subsubsection{Classification}

The classification of bHLH proteins can be based upon different properties, such as originating species, phylogenetic homology, ${ }^{3}$ typical spatial or temporal expression pattern, biochemical properties or regulated target genes. The popular classification system by Murre $^{4}$ is a pragmatic compromise:

- Class I bHLH transcription factors (E-proteins such as Tcf3 of Tcf4) are ubiquitously expressed. They are able to form homo- or heterodimers and generally act as transcriptional activators.

- Class II forms a very large group of tissue-specifically expressed bHLH proteins. Many of those have been reported to regulate cell-intrinsic differentiation processes. They form heterodimers with ubiquitously expressed

\footnotetext{
${ }^{1}$ Voronova and Baltimore 1990, fig 2: Site-directed mutation of the E47S bHLH domain

${ }^{2}$ Longo et al. 2008, fig 4, 7: Crystal structure of a complex of recombinant Tcf3 protein, Neurod1 protein, and a short double stranded DNA fragment coding for the E-box sequence of the rat insulin promoter.

${ }^{3}$ Atchley and Fitch 1997; Ledent et al. 2002

${ }^{4}$ Murre et al. 1994, fig 1 "Helix-loop-helix proteins categorized into different classes"
} 
class I bHLH proteins and control the tissue-specific expression pattern of other genes. Well known examples include MyoD, Myf5 and myogenin, which regulate muscle differentiation. Class II bHLH proteins expressed in the brain include the Neurogenins, the Oligs and the NeuroD-family.

- Class III comprises Myc related proteins. They are involved in growth control and play an important role in cancer.

- Class IV is a small group of proteins interacting with Myc.

- Class V HLH proteins lack the basic (DNA binding) domain. They act as competitive antagonists of other bHLH proteins by forming non-functional heterodimers. Examples are the Id proteins.

- Members of Class VI are characterized by high homology to the Drosophila protein 'Hairy and Enhancer of split'. A famous example is the negative regulator Hes5, the main effector of Delta-Notch signaling.

Neurod $1 / 2 / 6$, the proteins studied during this work, are mainly expressed in the CNS and belong to the large group of class II. They heterodimerize with ubiquitously expressed Class I bHLH factors (eg Tcf4) or with the dominant negative members of Class $V$ (eg Id2).

\subsubsection{Functions}

Several bHLH proteins were identified as master regulators of tissue-specific determination and differentiation processes. ${ }^{1}$ An ideal master regulator genes has the following two characteristics ${ }^{2}$ :

1. Its expression is sufficient to transform undifferentiated cells to the natural differentiated state, without the need of exposing them to other inductive signals or environments. Experimentally, this can be tested by forced overexpression of a DNA construct in immature cells that do not express the gene endogenously.

2. Its expression is necessary for proper tissue differentiation, even within the natural environment. This can be tested by genetically inactivating the factor in cells or in a whole organism.

A classical master regulator is the eyeless gene, a Pax6-related homeodomain transcription factor of Drosophila melanogaster. Normal eyeless expression is a prerequisite for natural eye development, and ectopic expression can induce the formation of additional eyes at the wings or legs of the flies. ${ }^{3}$

A famous example of an imperfect master regulator is MyoD, a class II bHLH protein that can drive muscle cell differentiation in-vitro but is dispensable for normal muscle development in mice. Combined inactivation of MyoD and the closely related gene Myf5 in mice results in the agenesis of skeletal muscles. ${ }^{4}$

\footnotetext{
${ }^{1}$ Saba et al. 2005; Lee and Pfaff 2003

${ }^{2}$ Chan and Kyba 2013

${ }^{3}$ Baker 2001: Eye development in Drosophila melanogaster is a bit more complicated and also controlled by other genes. The inductive potential of eyeless is context dependent and embedded in a reinforced network of several interacting genes.

${ }^{4}$ Rudnicki et al. 1993: Double homozygous animals completely lack skeletal myocytes and myoblasts.
} 


\begin{tabular}{|c|c|c|c|c|}
\hline \multirow{15}{*}{$\begin{array}{c}\text { atonal } \\
\text { (drosophila) }\end{array}$} & Neurod1 & NeuroD & Beta2, Bhf1 & bHLHa3 \\
\hline & Neurod2 & NDRF & & bHLHa1 \\
\hline & Neurod6 & NEX & Math2 & bHLHa2 \\
\hline & Neurod4 & NeuroM & Math3 & bHLHa4 \\
\hline & Neurog1 & Neurod3 & Math4C & bHLHa6 \\
\hline & Neurog2 & & Math4A, Atoh4 & bHLHa8 \\
\hline & Neurog3 & & Math4B, Atoh5 & bHLHa7 \\
\hline & Bhlhe22 & Beta3 & & bHLHb5 \\
\hline & Olig1 & & & bHLHb6 \\
\hline & Olig2 & & & bHLHb1 \\
\hline & Olig3 & & & bHLHb7 \\
\hline & Bhlha15 & Mist1 & & bHLHb8 \\
\hline & Atoh7 & & Math5 & bHLHa13 \\
\hline & Atoh1 & & Math1 & bHLHa14 \\
\hline & Atoh8 & Okadin & Math6 & bHLHa21 \\
\hline
\end{tabular}

Figure 7: Overview of atonal related bHLH proteins in mice

Phylogenetic analysis of mouse bHLH genes related to the Drosophila gene atonal (ato). The alignment was calculated using the complete coding sequences. The tree has been rooted using atonal as outgroup. Neurod5 (Atoh6) was excluded because only a partial sequence has been published thus far. The official gene symbols (MGI) are listed in the first column, followed by alternative and historic names. The NeuroD-family members studied here are highlighted in red/yellow.

This implies that not a single gene but a small set of genes must be considered as collective master regulator of muscle cell differentiation and muscle development.

For many developmental processes it is currently not clear whether they are regulated by individual master regulator genes, or by the collective state of large non-hierarchical genetic networks, or rather by a combination of both. ${ }^{1}$

\subsubsection{Neuronal bHLH Proteins}

Many bHLH genes are involved in neuronal development. Depending on their primary function, they can be divided into two overlapping groups:

Determination factors are expressed by multipotent precursor cells. They determine the principle cellular fate and initiate for example glial or neuronal differentiation. Neuronal determinators are called proneural factors. Examples include the Neurogenins (Neurog1/2), Ascl1 (Mash1), Neurod4 and Neurod1.2

Differentiation factors are expressed by already determined cells and regulate terminal differentiation. Those expressed in committed neurons are called neuronal bHLH proteins. Examples include Neurod1/2/6,Bhlhe22 and Cux $1 .{ }^{3}$

\footnotetext{
1 Bar-Yam et al. 2009

${ }^{2}$ Bertrand et al. 2002 (review); Ma et al. 1996 (Neurog1); Tomita et al. 2000 (Ascl1, Neurod4); Roztocil et al. 1997 (Neurod4); Lee et al. 1995 (Neurod1)

${ }^{3}$ Bertrand et al. 2002 (review); Pleasure et al. 2000 (Neurod2); Schwab et al. 2000 (Neurod1, Neurod6); Joshi et al. 2008 (Bhlhe22); Cubelos et al. 2010 (Cux1)
} 


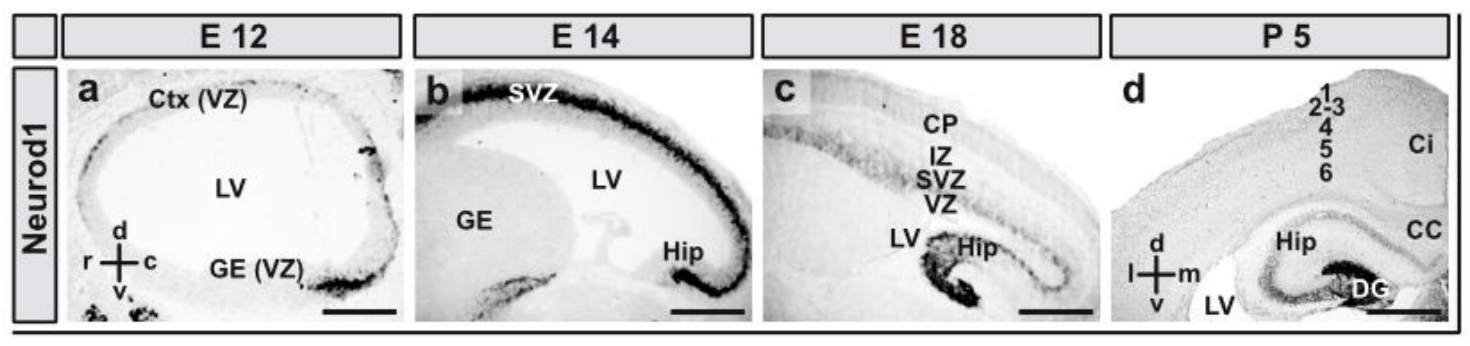

Figure 8: Neurod1 expression in the developing cerebral cortex

In situ hybridization for Neurod1 mRNA at (a) E12, (b) E14 and (c) E18 in sagittal plane and (d) P5 in coronal plane. In-situ hybridization was performed by Maike Gummert, Department of Neurogenetics. Details in Gummert 2003 and Bormuth et al. 2013.

\subsection{The NeuroD-Family}

$\mathrm{T}$ HE NEUROD-FAMILY comprises four closely related neuronal bHLH transcription factors: Neurod1/2/4/6 (fig 7). These have long been suspected to play important roles in pyramidal neuron differentiation and embryonic cortex development. $^{1}$

\subsubsection{Neurod1}

The onset of cortical Neurod $1^{2}$ expression in mice is around embryonic day (E) 11 , when the first pyramidal neuron precursors leave the $\mathrm{VZ}$ and undergo neuronal differentiation (fig 8a). In the developing cerebral cortex, Neurodl is predominantly expressed in mitotic and early-postmitotic neuronal progenitor cells that are located in the SVZ. The embryonic CP, which comprises post-mitotic and at least basally differentiated neurons, is negative for Neurod1 (fig 8b,c). After birth, Neurod 1 is strongly expressed by most pyramidal neurons located in the upper neocortical layers, but mostly absent from deeper layers. ${ }^{3}$ The

${ }^{1}$ Lee et al. 1995: Cloning of the mouse Neurod1 gene and neuronal conversion of Xenopus ectoderm by overexpression of Neurod 1 ("the data suggest that NeuroD may participate in the terminal differentiation step during vertebrate neuronal development"); Kawakami et al. 1996: Cloning and expression analysis of the rat Neurod 1 (BHF-1) gene ("bHLH factors are believed to play essential roles in neural development and neural functions"); Yasunami et al. 1996: Cloning and characterization of the mouse Neurod2 (NDRF) gene (the authors suggest "distinct roles in neural development and differentiation"); Kume et al. 1996: Cloning and expression analysis of the rat Neurod2 (KW8) gene ("several bHLH proteins have been identified that may play essential roles in neurogenesis or neural development”); Franklin et al. 2001: Expression analysis of Neurod 1 and Neurod2 during human brain development ("Neurod1 and Neurod2 appear more likely to play a role in neuronal differentiation")

${ }^{2}$ Lee et al. 1995: Neurod1 was initially cloned as NeuroD from a mouse embryonic stem cell tumor cDNA library that was screened for interaction with the Drosophila bHLH transcription factor daughterless (da); Naya et al. 1995 Neurod 1 was independently cloned as Beta2 from a hamster insulin tumor cDNA library that was screened for interaction with Tcf3 (E47) and for binding to the insulin promoter; Kawakami et al. 1996: Neurod 1 was independently cloned as Bhf1 from a rat cerebellum cDNA library that was screened for similarities with a bovine genomic clone, which had been found by hybridizing a genomic library to the bovine myosin I heavy chain gene.

${ }^{3}$ Goebbels 2002, fig 18i: $\beta$-galactosidase histochemistry in adult heterozygous Neurod 1-LacZ mice. Only a very small subset of deeper layer neurons expresses Neurod1. Most of these cells are also Neurod6-positive. 
Neurod 1 expression domain is not CNS-specific ${ }^{1}$ but includes endocrine cells of the pancreas and in the intestine. ${ }^{2}$ Overexpression of Neurod 1 is sufficient to convert Xenopus ectoderm into neurons ${ }^{3}$ and to induce neurite outgrowth in neuroblastoma cell lines. ${ }^{4}$

\subsubsection{Neurod2 and Neurod6}

Neurod $2^{5}$ and Neurod6 ${ }^{6}$ are exclusively expressed in the $\mathrm{CNS}^{7}$ (fig 9; fig 13). Neurod2/6 expression domains include the cerebral cortex, hippocampus, olfactory bulb, amygdala, hypothalamus, pontine nucleus and the cerebellum. ${ }^{8}$

Onset of cortical Neurod2/6 expression is around E12 in mice, when the first pyramidal neurons migrate into the developing CP (fig 9a, e). During embryogenesis, both factors are expressed by postmitotic pyramidal neurons in the SVZ and CP (fig 9b, c, f, g). Neurod2/6 expression levels peek shortly after birth and subsequently decrease to a weaker level, which is maintained throughout adulthood. ${ }^{9}$ In the adult brain, neocortical Neurod6 expression is confined to a subset of pyramidal neurons that are predominantly located in deeper layers ${ }^{10}$ (fig 1a, e). Expression of Neurod2 is maintained at detectable levels in all layers of the CP. ${ }^{11}$

${ }^{1}$ Schwab et al. 1998, fig 6-7: In contrast to Neurd2/6, Neurod1 is strongly expressed in developing olfactory neuroepithelium, cranial ganglia and dorsal root ganglia. Neurod $1 / 2$, but not Neurod6 are expressed in most cerebellar granule neurons.

${ }^{2}$ Naya et al. 1997: X-gal staining in heterozygous Neurod1-LacZ mice demonstrates Neurod1 expression in insulin producing $\beta$-cells of the pancreas and in secretin expressing S-cells of the small intestine.

${ }^{3}$ Lee et al. 1995, fig 4, 5: Injection of Neurod 1 mRNA into Xenopus frog embryos induced expression of neuronal markers (Ncam $1, \beta$-tubulin and neurofilament).

${ }^{4}$ Noma et al. 1999, fig 4: Neurod1 overexpression induces NGF independent neuronal outgrowth in PC12 (rat pheochromocytoma) cells; Cho et al. 2001, fig 4: Neurod1 overexpression induces neuronal outgrowth in F11 cells. This is abolished by co-expression of a C-terminally truncated (dominant negative) form of Neurod 1.

${ }^{5}$ Kume et al. 1996: Neurod2 was initially cloned as KW8 from a cDNA subtraction library of native vs. TEA treated rat brain tissue; Yasunami et al. 1996: Neurod2 was independently cloned as NDRF from an embryonic mouse cDNA library using a rat Neurodl cDNA probe; McCormick et al. 1996: Neurod2 was independently cloned from an embryonic mouse cDNA library that was screened for similarities with a human genomic clone, which had been found by hybridizing a human fibroblast genomic library with a Neurod 1 probe.

${ }^{6}$ Bartholomä and Nave 1994: Neurod6 was initially cloned as NEX-1 from an adult rat brain library by PCR using degenerate primers targeting conserved regions in the bHLH domains of other known bHLH genes (c-myc, MyoD, myogenin); Shimizu et al. 1995: Neurod6 was independently cloned as Math2 from an embryonic rat brain cDNA library by PCR using degenerate primers targeting the Atonal gene of Drosophila melanogaster (ato).

${ }^{7}$ Schwab et al. 1998: ISH for Neurod1/2/6; Goebbels 2002 and Goebbels et al. 2006: Linage analysis using genomic recombination in Neurod6-Cre mice; Lin et al. 2004: Very sensitive expression analysis using Neurod2-LacZ mice.

${ }^{8}$ Lin et al. 2004, tab 1: Neurod2 expression domains; Goebbels 2002; Goebbels et al. 2006, tab 1: Neurod6 expression domains

${ }^{9}$ Schwab et al. 1998, fig 4: Northern blot analysis on total RNA of E12, E16, E20, P0, P5, P10, P15, P20 rat brains and adult cerebellum

${ }^{10}$ In the neocortex of adult mice, Neurod6-Cre is predominantly expressed in deeper layers. Most Neurod6-positive cells co-express Neurod2. Only a small subset co-expresses Neurod1. The majority of neocortical pyramidal neurons are Neurod6-negative. (Goebbels 2002, fig 18L; Gummert 2003, fig 14, 18, 19).

${ }^{11}$ Lin et al. 2004, fig 3: X-gal histochemistry in brain sections from adult heterozygous Neurod2LacZ mice 


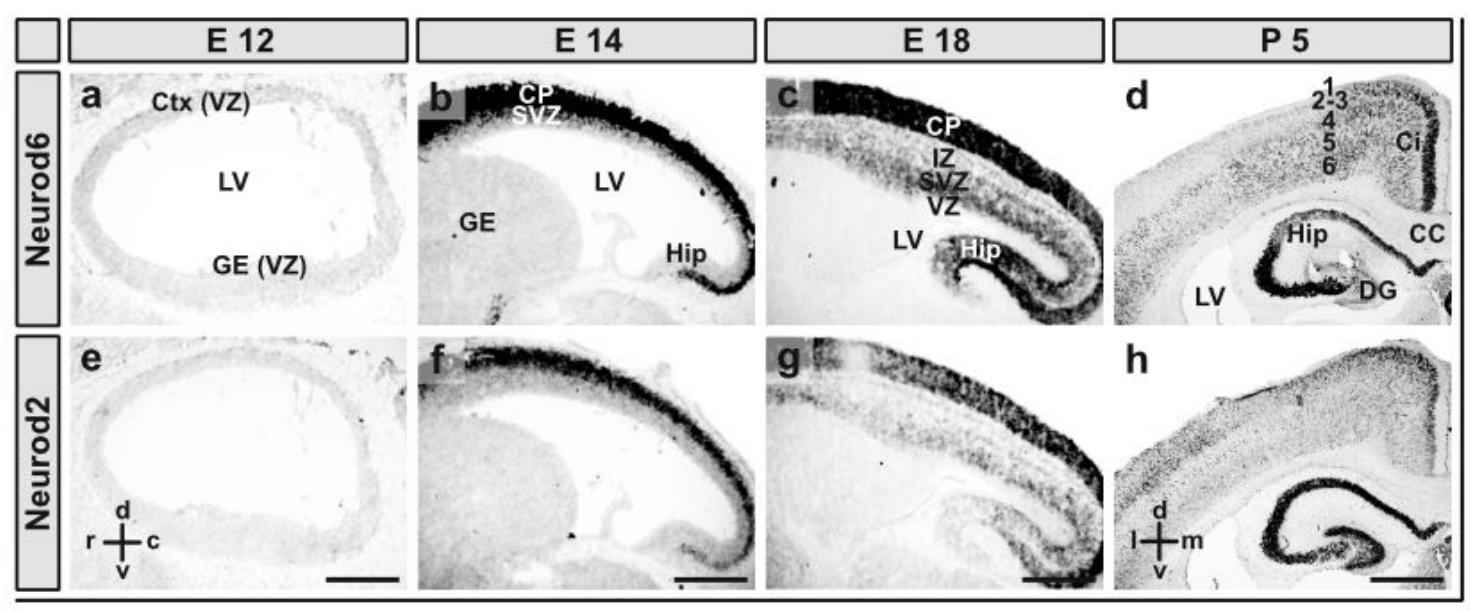

Figure 9: Neurod2 and Neurod6 expression in the developing cortex

In-situ hybridization for (a-d) Neurod2 mRNA and (e-h) Neurod6 mRNA at (a, e) E12, (b, f) E14 and (c, g) E18 in sagittal plane and (d, h) P 5 in coronal plane. In-situ hybridization was performed by Maike Gummert, Department of Neurogenetics. Details in Gummert 2003 and Bormuth et al. 2013.

\subsubsection{Neurod4}

Neurod $4^{1}$ expression was initially described in the chicken spinal cord and optic tectum, where it is robustly expressed in a small time window between neurogenic cell division and the onset of Neurod 1 expression. ${ }^{2}$ Neurod 4 expression levels in the mouse neocortex are very low and not easily detectable by in-situ hybridization (ISH). ${ }^{3}$ Forced expression of Neurog2 or Neurod 1 results in transcriptional upregulation of Neurod4 in vivo; Neurog2 and Neurod4 can synergistically induce Neurod 1 expression. ${ }^{4}$

\subsection{Genetic Inactivation Studies}

$\mathrm{I}$ N THE PAST, Neurod1, Neurod2 and Neurod6 have been genetically inactivated in mice. However, none of the mutations resulted in striking abnormalities of embryonic pyramidal neuron differentiation:

\footnotetext{
${ }^{1}$ Peyton et al. 1996: Neurod4 was initially cloned as Beta3 from a hamster insulin tumor cDNA library by PCR using partially degenerate primers that binding the bHLH domains of other class II bHLH genes.

${ }^{2}$ Roztocil et al. 1997: Robust Neurod4 expression was detected in chicken retina, diencephalon, metencephalon, spinal cord and dorsal root ganglia, but not in the telencephalon.

${ }^{3}$ Chromogenic ISH showed moderate Neurod4 expression in the hippocampus and weak Neurod4 expression in the neocortex of E14 mice (Gummert 2003, fig 3I). Double ISH for Neurog2 and Neurod4 showed detectable but comparability low Neurod4 expression at E13.5 (Mattar et al. 2008, fig 1B, Q). We performed quantitative expression analysis of E13 mouse neocortex that identified Neurod4 transcripts at a level that was approximately 20 times lower than that of Neurod2 (data not shown).

${ }^{4}$ Mattar et al. 2008, fig 5-7. The authors "implicate Math3 as a key cofactor in the Neurog2regulated cortical differentiation cascade“"
} 


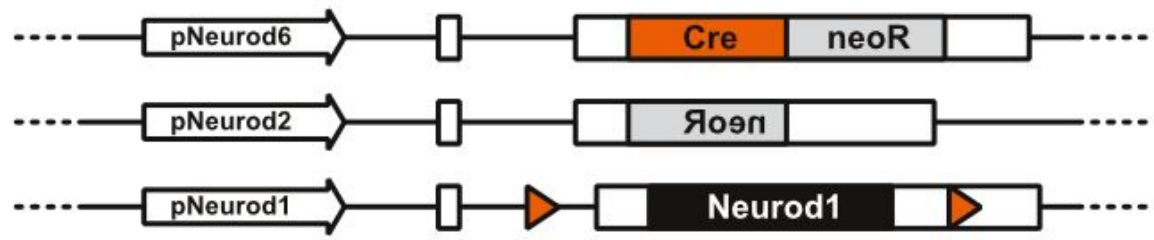

Figure 10: Used transgenic alleles for Neurod1, Neurod2 and Neurod6

Schematic illustration of the mutated loci after homologous recombination in embryonic stem cells. (a) Neurod1-Flox by Goebbels et al. 2005. (b) Neurod2-Null by Yonemasu et al., published in Bormuth et al. 2013. (c) Neurod6-Cre by Goebbels et al. 2006.

\subsubsection{Inactivation of Neurod1}

Neurod 1 was originally inactivated by Tsai and colleagues ${ }^{1}$ with a primary focus on the pathogenesis of diabetes mellitus. Their mutant animals suffered from severe hyperglycemia combined with ketonuria (typical signs of diabetes) and died within days after birth. At this age, the number of insulin producing $\beta$-cells was reduced by $74 \%$ and apoptosis was increased 14 -fold. The authors did not describe obvious defects in the nervous system.

Subsequently, Lee and colleagues analyzed Neurod 1 mutant mice with emphasis on the CNS. In order to rescue diabetes induced lethal, they additionally introduced a transgene expressing Neurod 1 under control of the rat insulin promoter. ${ }^{2}$ Neurod 1 deficient mice carrying the transgene survived about one month after birth. They showed retarded body growth and developed a severely ataxic gait. The cerebellum was smaller and the number of cerebellar granule neurons was reduced by $60-70 \%$. While pyramidal neurons of the cerebral cortex and hippocampal pyramidal neurons were not affected, hippocampal granule cells died from apoptosis, which resulted in agenesis of the dentate gyrus (DG). A later study showed that the loss of granule cells was due to increased developmental cell death that could be prevented by genetic inactivation of the pro-apoptotic $\mathrm{Bcl} 2$ associated protein $\mathrm{X}(\mathrm{Bax}) .^{3}$

The group of Tsai found that outbreeding their mutant mice into the $129 \mathrm{X} 1 / \mathrm{SvJ}$ (129X1) genetic background ${ }^{4}$ could generate weakly diabetic Neurod 1 mutants that survived in most cases. ${ }^{5}$ Adult mutants showed severe ataxia, hyperactivity, circling, swaying head movements and epileptic seizures. They confirmed the

\footnotetext{
${ }^{1}$ Naya et al. 1997, fig 1: The entire ORF was replaced by the lac $Z$ coding sequence.

${ }^{2}$ Miyata et al. 1999, fig 1: The mouse Neurod 1 coding region is driven by a $\sim 700$ bp fragment of the rat insulin promoter

${ }^{3}$ Kim et al. 2012: Bax inactivation in Neurod 1 deficient mice prevented apoptosis in the hippocampal DG, the cerebellar external granular layer, and the vestibular and cochlear cranial nerve nuclei. Previously described defects in neuronal migration, however, could not be rescued. Independently of the presence of Bax, Neurod 1 deficient mice died within days after birth.

${ }^{4}$ Simpson et al. 1997, fig 2: overview of 129 sub-strains; Naming of mouse strains in this work follows the "Guidelines for Nomenclature of Mouse and Rat Strains" by the international committee on standardized genetic nomenclature: http://jaxmice.jax.org/support/nomenclature/bulletin01.html

${ }^{5}$ Liu et al. 2000: Neurod 1-LacZ mice were generated in a 129/SvEv (129S) genetic background and showed neonatal lethality. Outbreeding to C57BL/6J did not change survival rates. However, outbreeding the C57BL6/J X 129/SvEv hybrid mice to 129X1/SvJ (129X1, formerly $129 / \mathrm{SvJ})$, resulted in 60-70 \% of homozygous mutants to survive to adulthood.
} 
lack of an organized DG but identified a small cap of surviving granule cells in close proximity of the hippocampal CA4.

Recently, conditional Neurod 1 deficient mice have been generated in our department by Sandra Göbbels (Neurod1-Flox). ${ }^{1}$ The expression of Neurod 1 is not changed under normal conditions, but the presence of Cre recombinase results in the permanently deletion of Neurod 1 from all Cre-positive cells (sect 1.7). In a prove-of-principal experiment, Neurod1-Flox mice were bred to mice expressing Cre under control of the $\mathrm{GABA}_{\mathrm{A}}$ receptor $\alpha 6$ subunit promoter. This promoter is selectively active in postmitotic, postmigratory granule neurons of the cerebellum, starting 1 week after birth. ${ }^{2}$ Offspring of the genotype Neurod $1^{\text {Flox } / \text { Flox }} \times a 6^{\text {Wt } / \text { Cre }}$ entirely lacked Neurod 1 messenger RNA (mRNA) and Protein from cerebellum while Neurod 1 levels in other tissues were unchanged.

During this work, conditional Neurod1-Flox mice were used in combination with Neurod6-Cre mice to selectively inactivate Neurod 1 in committed pyramidal neurons (sect 1.5.3; fig 10).

\subsubsection{Inactivation of Neurod2}

The genetic inactivation of Neurod 2 in mice was initially published by Olson and colleagues who replaced the complete open reading frame (ORF) with the coding sequence of $\mathrm{E}$. coli b-galactosidase (lacZ). ${ }^{3}$ These animals developed normally until two weeks after birth, when they began to suffer from ataxia and failure to thrive. The thickness of the cerebellar granule cell layer was reduced by about $25 \%$, and apoptosis rates in that region were increased fivefold at postnatal day (P) 19. Expression of brain derived neurotrophic factor (BDNF) and medium neurofilament (Nefm) was decreased. Homozygous mice died between two to four weeks after birth (around the time of weaning).

Neurod2 is also involved in amygdala development. Heterozygous Neurod2-LacZ mice lack the lateral and basolateral nucleus of the amygdala and show reduced fear levels and impaired emotional learning in unconditioned and conditioned fear tests. Amongst the strongest downregulated genes were Ulip1, AMPA and GABA receptors. ${ }^{4}$

Another study showed that Neurod2 deficient mice suffer from severe hypothyroidism. Neurod2 is normally expressed in the periventricular nucleus of the hypothalamus (PVN), the anterior pituitary and the thyroid gland. The levels of thyreotropin releasing hormone (TRH), thyroid-stimulating hormone (TSH) and thyroxine (T4) were strongly reduced in Neurod2 deficient mice. ${ }^{5}$

\footnotetext{
${ }^{1}$ Goebbels et al. 2005, fig 1: The entire Neurod 1 ORF was flanked by LoxP sites.

${ }^{2}$ Fünfschilling and Reichardt 2002; Goebbels et al. 2005, fig 2d-f

${ }^{3}$ Olson et al. 2001, fig 1 (the targeting vector introduced a floxed neomycin resistance cassette that was removed by genomic recombination in ES cells)

${ }^{4}$ Lin et al. 2005: Heterozygous Neurod2-LacZ mice in both 129/Sv and C57BL/6 genetic background were tested in open arm of elevated plus maze, light dark box, and freezing response tests.

${ }^{5}$ Lin et al. 2006: X-gal histochemistry demonstrates Neurod2 expression in all components of the hypothalamic-pituitary-thyroid axis. Serum thyroxine levels were reduced by more than $50 \%$. Parenteral thyroxine substitution starting from E7 increased the mean lifespan from 14 to 55 days.
} 
Neurod2 function is essential for postnatal refinement of the somatosensory cortex. In Neurod2 deficient mice, thalamic axons grow normally into the neocortex and form synaptic contacts with pyramidal neurons in layer 4 . However, maturation of thalamocortical synapses and activity-dependent formation of the typical whisker-barrels are severely disturbed in the absence of Neurod2. ${ }^{1}$ InceDunn et al. 2006 mention that "the CC failed to form in Neurod2 heterozygous and null mice". The latter was not supported by histological evidence ${ }^{2}$ and the $\mathrm{CC}$ is clearly visible in published histological data of heterozygous Neurod2-LacZ mice $^{3}$ (fig 17).

Neurod2 deficient mice were independently generated in the Department of Neurogenetics by Tomoko Yonemasu. She replaced the entire ORF with a reversely-oriented neomycin resistance cassette (Neurod2-Null, fig 15). This excludes potential toxic effects of $\beta$-galactosidase expression, but does not allow to monitor Neurod2 promoter activity in homozygous mutant mice. ${ }^{4}$ Neurod2Null mice were used during this study. A brief phenotypical overview is provided in sect 2.3 .

In conclusion, Neurod2 functions have been identified in different neuronal tissues and during synapse formation of postnatal cortical pyramidal neurons, however, embryonic pyramidal neuron differentiation and cortex development seem largely unaffected by the loss of Neurod2.

\subsubsection{Inactivation of Neurod6}

Neurod6 was originally inactivated by Markus Schwab who replaced the complete ORF located in exon 2 by a neomycin resistance cassette (Neurod6-Null). ${ }^{5}$ To the surprise of many, the absence of Neurod6 mice did not result in any obvious abnormalities.

Sandra Göbbels later created Neurod6-Cre mice by replacing the Neurod6 ORF with the coding sequence of Cre recombinase ${ }^{6}$ (fig 10). The phenotype of these animals resembles that of the conventional Neurod6-Null mice. Neurod6-Cre mice allow for easy visualization of Neurod6-promoter activity by immunostaining for Cre. It is possible to permanently deactivate or activate other genes selectively in the Neurod6-lineage, in all cells that had ever expressed Neurod6 (sect 1.7). In this study, Neurod6-Cre mice were used to inactivate Neurod6 and to drive the conditional inactivation of Neurod 1 .

\footnotetext{
${ }^{1}$ Ince-Dunn et al. 2006, fig 8: The ratio of NMDA to AMPA mediated postsynaptic responses was approximately ten-fold increased in Neurod2 deficient mice at P12.

${ }^{2}$ Ince-Dunn et al. 2006, suppl. fig 2 shows coronal brain sections of P7 wild type, heterozygous and homozygous Neurod2-LacZ mice. However, the section plane of the latter two brains is more caudal, does not include midline glia structures, and might just have missed the CC.

${ }^{3}$ Olson et al. 2001, fig 3E; Lin et al. 2005, fig 4a (horizontal sections of heterozygous Neurod2$\mathrm{LacZ}$ mice). CC formation might be disturbed at incomplete penetrance or in dependence of the genetic background.

${ }^{4}$ Bormuth et al. 2013, fig 1i-k: The reversely orientated neomycin resistance cassette includes a promoter that is active in ES cells. Transcription initiated by the Neurod2-promoter does not result in any meaningful gene product.

${ }^{5}$ Schwab et al. 1998, fig 1a: The neomycin resistance cassette is expressed under control of the Neurod 2 promoter and can be used to monitor the transcriptional activity of the mutated allele at the RNA level (fig 3).

${ }^{6}$ Schwab et al. 2000; Goebbels et al. 2006
} 


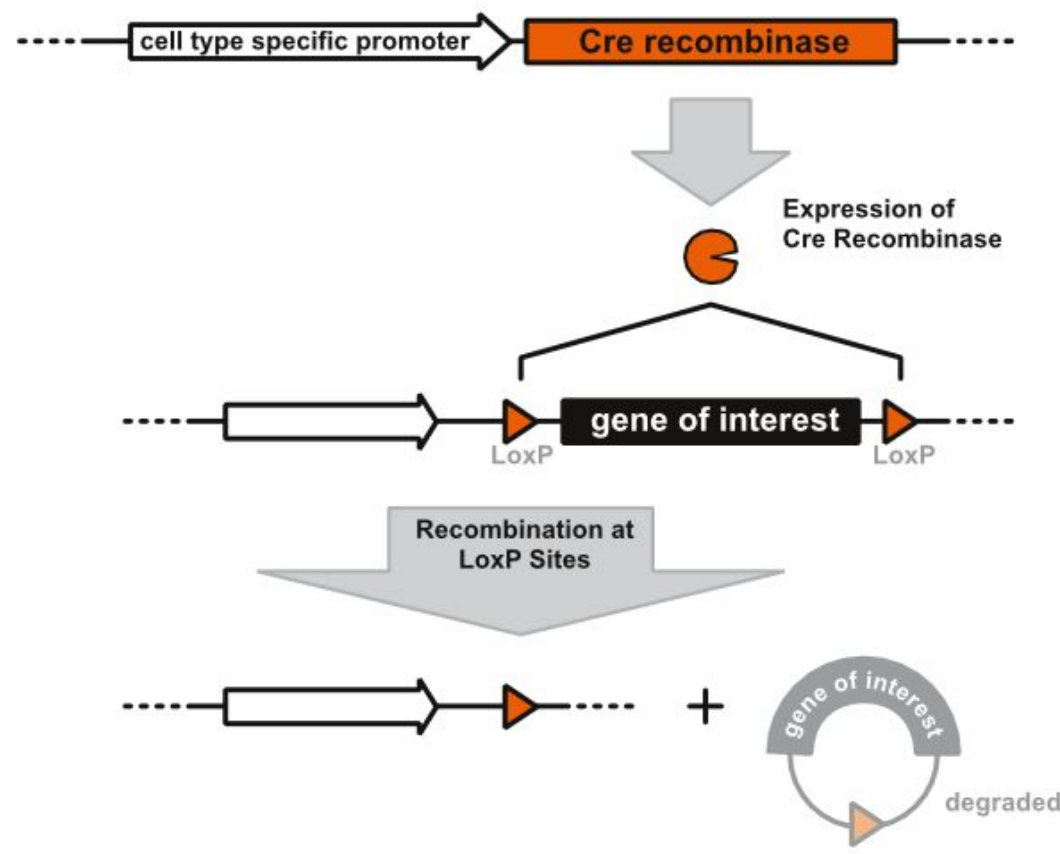

Figure 11: The principle of Cre-LoxP based gene inactivation

Classically, two different genetically modified mouse lines are generated. A driver line expresses Cre recombinase under control of a promoter that is selectively active in certain types of cells. A conditional mutant line, where important exons of some gene are flanked by LoxP sites. Once the mouse lines are crossbred, Cre recombinase catalyzes the non-reversible inactivation of the gene of interest only in the cells of interest.

\subsection{Functional Redundancy}

I N CONSIDERATION of the described single gene inactivation studies it must either be concluded that none of the NeuroD factors play important roles in differentiating pyramidal neurons, or that they have overlapping functions that can alternatively be provided by any of the genes. Such functional redundancy or compensation is a feature frequently observed in biology.

The high sequence similarity (at least in the bHLH domain, which mediates dimerization and DNA binding) and overlapping expression patterns suggest redundant functions between Neurod6, Neurod2 and (to a lesser extent) Neurod1. The canonical way to test whether two genes share common functionality is to simultaneously inactivate both, and to compare the outcome to that of each single gene inactivation.

First attempts in this direction had already been taken: When Markus Schwab generated Neurod6 deficient mice in 1998, he quickly realized that these animals did not show apparent abnormalities. ${ }^{1}$ Hypothesizing functional redundancy with Neurod 1 , he generated Neurod $1 / 6$ double-deficient mice. These died shortly after birth due to pancreatic failure caused by the loss of Neurod1. This study exposed a redundant role in terminal granule neuron differentiation, but neocortical

\footnotetext{
${ }^{1}$ Schwab et al. 1998
} 


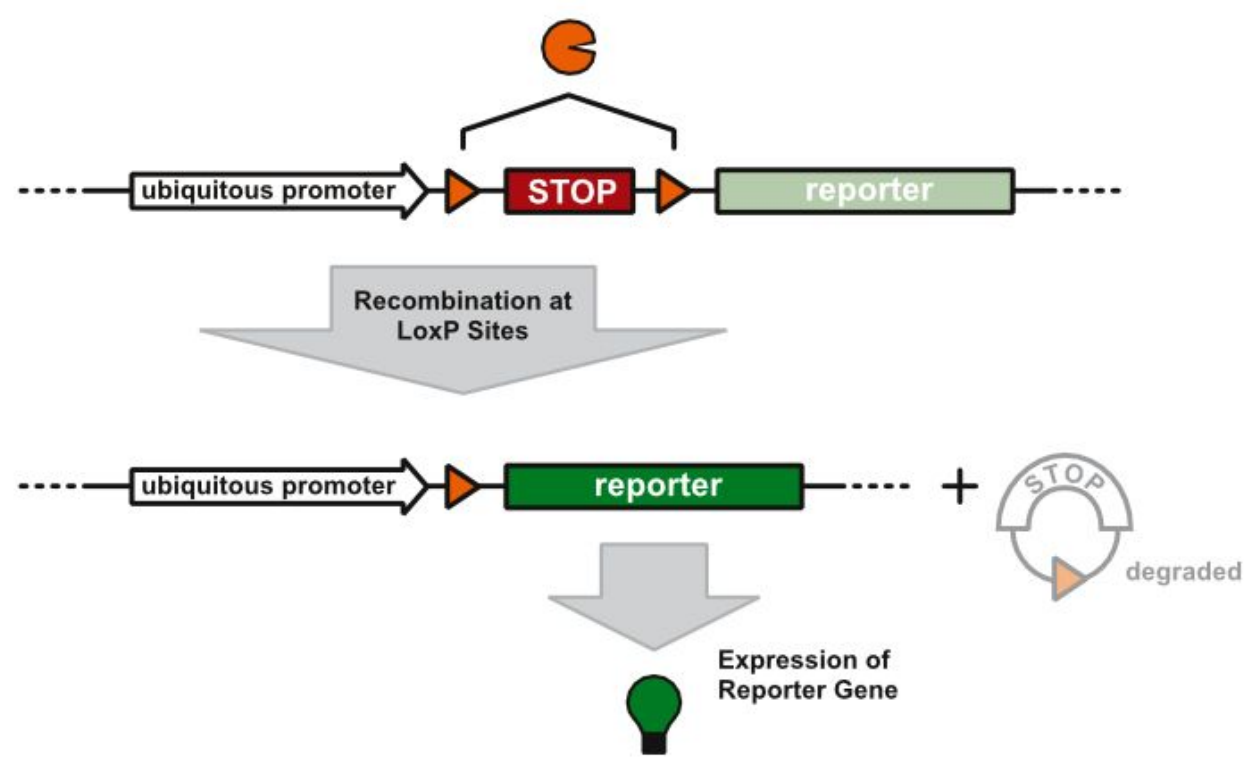

Figure 12: The principle of Cre-LoxP based gene activation

A variant of the scheme described in fig 11 is used to conditionally activate genes: An open reading frame including a STOP codon and ideally a poly $(\mathrm{A})$ tail is flanked by LoxP sites and put in-between a gene and its promoter. Cre mediated recombination removes the stop sequence and permanently activates the gene under the control of its endogenous promoter.

development was not noticeable affected by the loss Neurod $1 / 6{ }^{1}$ Neurod2 mutant mice were not available at that time. The generation of Neurod $1 / 2$ double-deficient mice was briefly mentioned in $2012,{ }^{2}$ but the phenotype of those animals has not been published yet.

\subsection{The Cre-LoxP System}

$\mathrm{T}$ HE CRE-LOXP SYSTEM is an advanced genetic tool isolated from bacteriophage P $1 .^{3}$ It was patented by Du Pont de Nemours and Company ${ }^{4}$ but was licensed for non-commercial purpose. ${ }^{5}$ The term Cre war originally an abbreviation for "causes recombination". The Cre protein is a tyrosine recombinase that can specifically cut and re-ligate DNA at LoxP sites $^{6}$ (locus of X-over P1).

\footnotetext{
${ }^{1}$ Schwab et al. 2000, fig 2: In comparison with Neurod 1 single-deficient animals, the overall hippocampal size and the number of hippocampal granule cells was further reduced in Neurod $1 / 6$ double-deficient mice.

${ }^{2}$ Kim et al. 2012: Nscl1-expression, which was decreased in cerebellar granule cells of Neurod 1 single-deficient mice, was not further reduced upon additional inactivation of Neurod2. The author used conventional Neurod 1-LacZ and Neurod2-LacZ mice and did not provide any data: "The detailed phenotype of the double KO mice will be reported elsewhere."

${ }^{3}$ Rossant and McMahon 1999: Report of the workshop for "Conditional Genome Alterations" held 1998 in Cold Spring Harbor

${ }^{4}$ US patent number 4959317 "Site-specific recombination of DNA in eukaryotic cells": http://google.com/patents?id=6DgiAAAAEBAJ

${ }^{5}$ The DuPont Cre-Lox License: http://patron.ucop.edu/ottmemos/docs/ott98-05.html

${ }^{6}$ Nagy 2000: Review on different application of the Cre-LoxP system in mouse genetics
} 
The Cre-LoxP system is used by geneticists to inactivate or activate genes in certain cells of living organisms (fig 11 and fig 12, respectively). In these regimes, Cre recombinase permanently removes LoxP-site flanked DNA sequences from the genome. Recombined alleles are inherited to all progeny of transiently Crepositive cells. Cre mediated recombination is widely used for cell lineage tracing and for conditional inactivation of genes in certain cell lineages.

A large number of Cre driver lines and conditional mutant mice exist today. ${ }^{1}$ The International Knockout Mouse Consortium (IKMC) ${ }^{2}$ has the goal to conditionally inactivate all known protein-coding genes in mice. In principle, it is possible to inactivate any gene in any cell type that is defined by the activity of a single promoter.

\footnotetext{
${ }^{1}$ Database of mouse Cre driver lines: http://nagy.mshri.on.ca/cre/

${ }^{2}$ The IKMC is part of the International Mouse Phenotyping Consortium (IMPC): http://mousephenotype.org
} 


\section{Results}

It is from the progeny of this parent cell that we all take our looks; we still share genes around, and the resemblance of the enzymes of grasses to those of whales is in fact a family resemblance.

Lewis Thomas ${ }^{1}$

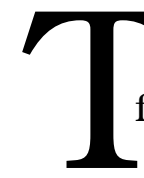

HIS WORK AIMS to identify and understand biological functions of NeuroDfamily transcription factors in pyramidal neuron differentiation and cortex development. Neurod1, Neurod2 and Neurod6 have previously been inactivated in mice. However, single gene inactivation did not significantly impact embryonic pyramidal neuron differentiation ${ }^{2}$ (sect 1.5). During this study, all possible compound mutants were generated and analyzed with a focus on cerebral cortex development and connectivity. The most relevant results can be summarized as follows:

1. Functions of Neurod $1 / 2 / 6$ are highly redundant in pyramidal neurons and can often be provided by any of the three proteins.

2. Guided axon growth towards distant targets, the outstanding feature of neocortical pyramidal neurons, depends on a genetic program that is initiated mainly by Neurod2/6. Accordingly, all callosal and most subcortical fiber tracts failed to reach their targets in Neurod2/6 double-deficient mice. This is a specific defect, as the majority of double mutant pyramidal neurons showed relatively inconspicuous migration patterns, molecular identity, and initial axon outgrowth.

3. Neocortical arealization and determination of the somatosensory cortex are functions of Neurod2/6. In Neurod2/6 double-deficient mice, pyramidal neurons of the putative somatosensory cortex area failed to acquire the typical molecular identity. The activity dependent formation of whiskerbarrels, which had been already reduced in Neurod2 single-deficient mice (sect 1.5.2), was entirely absent upon additional inactivation of Neurod6.

4. Terminal differentiation and subtype-specification of pyramidal neurons, while not strictly dependent on Neurod2/6, require functions of NeuroDfamily transcription factors. In the neocortex of Neurod $2 / 6$ double-deficient

\footnotetext{
${ }^{1}$ Thomas 1974, p 3, chapter The Lives of a Cell

${ }^{2}$ The genetic inactivation of neither Neurod1 (Miyata et al. 1999; Liu et al. 2000), Neurod2 (Olson et al. 2001; Ince-Dunn et al. 2006), Neurod6 (Schwab et al. 1998; Goebbels et al. 2006), nor Neurod1 and Neurod6 (Schwab et al. 2000) did result in obvious defects of neocortical pyramidal neuron differentiation during embryonic development (sect 1.5). The inactivation of Neurod2, however, affected the postnatal specialization of the somatosensory whisker-barrel cortex (Ince-Dunn et al. 2006).
} 


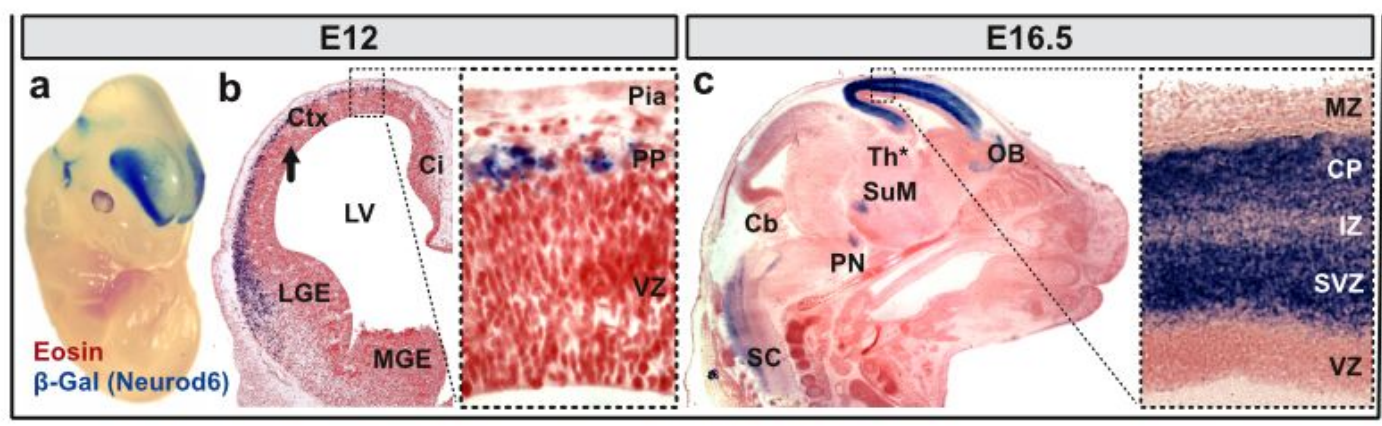

Figure 13: Neocortical Neurod6 expression is confined to the SVZ and CP

X-gal histochemistry (blue) marks all cells of the Neurod6-lineage (all cells that ever had expressed Cre from the Neurod6 promoter and thus underwent genetic recombination of the reporter allele). (a) E12 whole mount embryo in beveled ventral view. (b) E12 coronal brain section and (c) E16.5 parasagittal section of the head region. The arrow denotes the Neurod6-negative VZ. Boxed areas are show at higher magnification. Sections are counter stained with eosin (red).

mice, Neurod 1 expression, which is normally restricted to the subventricular zone, was maintained in the cortical plate and was sufficient to initiate most aspects of pyramidal neuron differentiation. The genetic removal of that ectopic Neurod 1 expression domain resulted in incomplete differentiation of pyramidal neurons, aberrant radial migration, and the loss of most cortical connectivity.

5. Differentiation and survival of hippocampal pyramidal neurons are functions of Neurod 1/2/6. In the hippocampus of triple-deficient mice, postmitotic neurons did not reach the pyramidal neuron state, but instead became apoptotic shortly after leaving the VZ.

\subsection{The Neurod6-Lineage of Cells}

$\mathrm{T}$ HE CLAIM that cortical Neurod6 expression is confined to postmitotic neurons (sect 1.4.2) was originally based on the absence of Neurod6 transcripts from the VZ, as shown by in situ hybridization. ${ }^{1}$ The lack of good Neurod6 antibodies hindered more sensitive analyses by immunohistochemistry for a long time. With the generation of Neurod6-Cre (NEX-Cre) mice ${ }^{2}$ (fig 10c), it became possible to indirectly visualize Neurod6 expression by using transgenic Cre reporter mice (fig 13) or Cre immunohistochemistry (fig 14) in heterozygous animals.

Blue staining in fig 13 visualizes the Neurod6-lineage of cells (sect 1.5.3) at the beginning and at the end of neocortical neurogenesis at E12 and E16.5, respectively. Neurod6-Cre mice had been crossbred with the Cre-reporter

\footnotetext{
${ }^{1}$ Bartholomä and Nave 1994, fig 4; Shimizu et al. 1995, fig 5; Schwab et al. 1998, fig 5; Taelman et al. 2001, fig 4 (Xenopus)

${ }^{2}$ Schwab et al. 2000, fig la; Goebbels 2002, sect II.1; Goebbels et al. 2006: Neurod6-Cre (NEXCre) mice express Cre recombinase under transcriptional control of the endogenous Neurod6 promoter.
} 


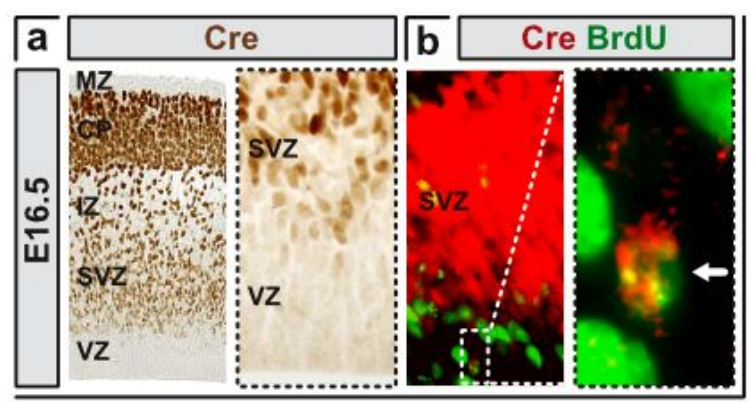

Figure 14: Neurod6 is mostly expressed by postmitotic pyramidal neurons

Sagittal section from the neocortex of a heterozygous Neurod6-Cre mouse at E16.5. (a) Immunohistochemical staining for Cre (brown) resembles Neurod6 expression (sect 1.5.3). (b) Double immunofluorescence for Cre (red) and BrdU (green) visualizes a small population of mitotic cells that still express Cre from the Neurod6 promoter. The arrow denotes one of the few Cre/BrdU double-positive cell. BrdU was administered to the pregnant female $4 \mathrm{~h}$ before the embryonic tissue was fixed.

line RosaStopLacZ (R26B). ${ }^{1}$ In double heterozygous animals, the expression of Cre recombinase is controlled by the endogenous Neurod6 promoter and results in genetic recombination of the reporter locus (sect 1.7) and permanent $\beta$-galactosidase expression in all cells that had ever expressed Cre. Blue staining originates from X-gal histochemistry, a sensitive method for the detection of $\beta$-galactosidase enzyme activity (sect 4.7.3). At E12, the beginning of cerebral cortex development, Neurod6-Cre expression was clearly confined to the first radially migrating pyramidal neurons of the neocortex (fig 13b). At E 16.5, the end of the neurogenic period, most cells in the CP and SVZ were lacZ-positive and thus part of the Neurod6-lineage (fig 13c). Cre expression (fig 14a) and Cre mediated recombination (fig 13) were never observed in the VZ, indicating that Neurod6 expression commences only after pyramidal neuron precursors leave the germinal cell layer. Germ line recombination was never observed in Neurod6-Cre mice.

For fig 14b, dividing cells were labeled by injecting E16.5 pregnant mice with bromodeoxyuridine (BrdU). The embryonic tissue was fixed four hours later and double immunofluorescence for Cre and BrdU demonstrated that the vast majority of mitotic cells did not express Neurod6. However, a small subset of clearly BrdU-positive cells located in the lower SVZ expressed Cre from the Neurod6 promoter at very low levels (arrow in fig 14b). This confirms the existence of a small subpopulation of mitotic Neurod6-positive cells that are located in the SVZ and presumably correspond to intermediate progenitors of neocortical projection neurons. $^{2}$

While Neurod6 was expressed by most postmitotic cells in the dorsal telencephalon, it was entirely absent from the ventral telencephalon and thalamus

\footnotetext{
${ }^{1}$ Soriano 1999, fig 1: An artificial sequence of a LoxP site, a neomycin resistance cassette, several polyadenylation sequences, another LoxP site, and a lac $Z$ ( $\beta$-galactosidase) gene was introduced into the ubiquitously expressed Rosa26 locus.

${ }^{2}$ Wu et al. 2005: A subset of Neurod6 expressing cells can still undergo symmetric and asymmetric divisions. At E15, $14.3 \%$ of the Neurod6-Cre positive cells located in the cortical SVZ or IC expressed the pan-proliferative antigen Ki-67 (fig 2d); $1.2 \%$ were positive for mitotic marker phosphohistone-H3 (fig 2d).
} 


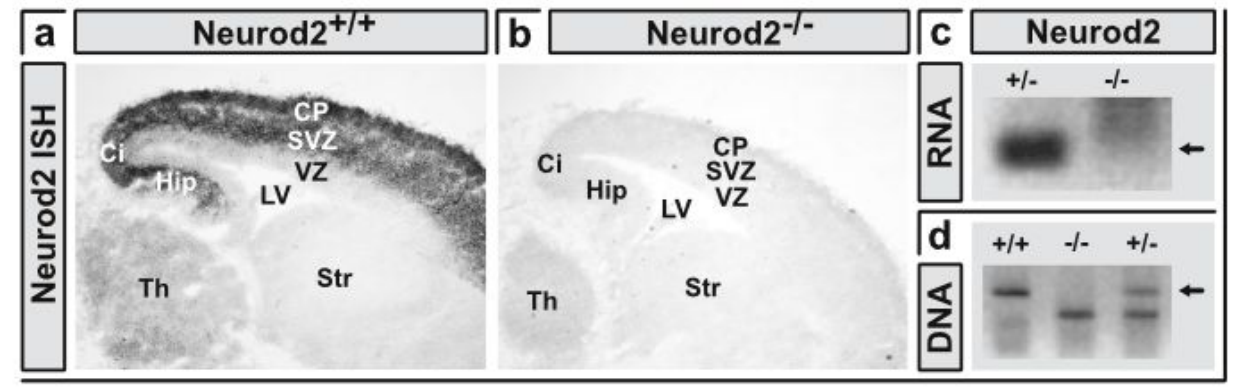

Figure 15: Absence of Neurod2 transcripts in Neurod2-Null mice

(a) In situ hybridization using a full length Neurod2 probe on sagittal cryostat sections of E14 wild type and homozygous Neurod2-Null mice. (b) Neurod2 transcripts were not detectable by RT-PCR in cortex tissue from E19 homozygous Neurod2-Null mice. (c) The Neurod2-Null genotyping PCR (sect 4.2.5) was routinely used to test for the genomic absence of the Neurod2 ORF. Arrows mark amplicons from the Neurod2 wild type allele (971 bp in c, 48 bp in d). Primer sequences (b) and (c) are listed in fig 47 and tab 4, respectively. In-situ hybridization was performed together with Maike Gummert, Department of Neurogenetics. Details in Gummert 2003; Bormuth et al. 2013.

(fig 13c). Even weak or transient Cre expression would have resulted in permanent genetic recombination and thus strong X-gal staining.

\subsection{Inactivation of Neurod6}

$\mathrm{N}$ NEUROD6 WAS INITIALLY INACTIVATED IN MICE by Markus Schwab (Neurod6Null $^{1}$ ), later by Sandra Göbbels (Neurod6-Cre ${ }^{2}$ ), and recently by Amit Agarwal (Neurod6-CreERT2 ${ }^{3}$ ). The authors did not report structural or behavioral abnormalities in any of those mouse models and considered Neurod6 as not strictly necessary for normal brain development in mice. ${ }^{4}$

For this study, I used Neurod6-Cre mice as Neurod6 deficient animal model. Homozygous Neurod6-Cre mice were born at the expected Mendelian ratio and were phenotypically indistinguishable from wild type littermates. Adult males and females were fertile, showed grossly normal social behavior and had a normal life span. Extensive histological analysis of cerebral cortex tissue from embryonic, newborn and adult homozygous Neurod6-Cre mice did not identify any effect of the single gene inactivation. Especially the layering and the connectivity of the cerebral cortex were not found to be changed. Neurod6-Cre homozygous

\footnotetext{
${ }^{1}$ Schwab et al. 2000, fig la: Generation of Neurod6-Null ('knock-out') mice and characterization of conventional Neurod $1 / 6$ double-deficient animals

${ }^{2}$ Goebbels 2002: Doctoral thesis in German language, translated title: "Cell type-specific expression of recombinase Cre in the nervous system of mice”; Goebbels et al. 2006: Generation and characterization of Neurod6-Cre ('knock-in') mice

${ }^{3}$ Agarwal et al. 2012: Generation and characterization of Tamoxifen dependent Neurod6-CreERT2 (inducible 'knock-in') mice; CreERT2 is a fusion protein of Cre recombinase and a mutated form of the human estrogen receptor ligand binding domain (Feil et al. 1997)

${ }^{4}$ Schwab et al. 1998: "These studies suggested that neuronal differentiation and function of CNS neurons are primarily unimpaired [...] in the absence of NEX [Neurod6].”; Goebbels et al. 2006: "NEX-Cre [Neurod6-Cre] mutants did not display any obvious histological or behavioral abnormalities"; Agarwal et al. 2012: Neurod6 is "dispensable for brain development in homozygous Nex1 [Neurod6] mouse mutants"
} 


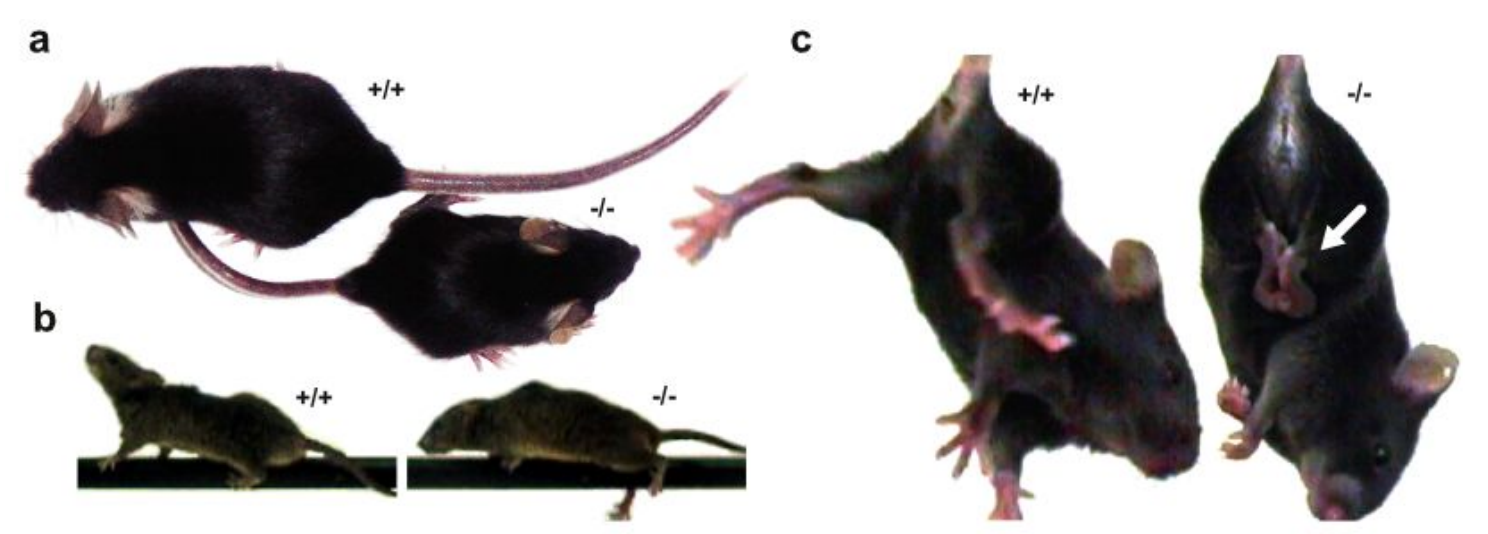

Figure 16: Phenotype of Neurod2-Null mice

Comparison of wild type (+/+) and homozygous Neurod2-Null (-/-) mouse littermates. (a) Obvious growth delay of adolescent Neurod2-Null mice. (b) Motor deficits in Neurod2-Null mice balancing on a bar. (c) Spontaneous limp-clasping (arrow) in adult Neurod2-Null mice. Pictures were extracted from video footage.

animals were often used as littermate controls for Neurod2/6 double-deficient or Neurod 1/2/6 triple-deficient mice (sect 2.4 and sect 2.5 ).

\subsection{Inactivation of Neurod2}

$\mathrm{N}$ EUROD2-NULL MICE were generated by Tomoko Yonemasu ${ }^{1}$ who replaced the entire Neurod2 ORF by a reversely-oriented neomycin resistance cassette (fig 10b). We confirmed the total absence of Neurod2 transcripts by ISH at E14 (fig 15a) and by quantitative reverse transcriptase PCR (qRT-PCR) at E19 (fig 15b), when Neurod2 expression normally reaches its maximum (fig 9).

As previously reported for Neurod2-LacZ mice, ${ }^{2}$ homozygous Neurod2-Null animals developed growth retardation (fig 16a), motor coordination deficits (fig 16b), and limb-clasping ${ }^{3}$ (fig 16c). All symptoms were comparatively mild and ameliorated over time. Most heterozygous and older homozygous animals were hardly distinguishable from wild type littermates.

\subsubsection{Lethality}

A subset of homozygous Neurod2-Null mice died around the fourth week after birth, at the time of weaning. Surviving animals slowly gained weight and were comparable to heterozygous or wild type littermates after the second postnatal

\footnotetext{
${ }^{1}$ Bormuth et al. 2013: First publication and short description of the Neurod2-Null allele

${ }^{2}$ Olson et al. 2001, fig 2: Homozygous (and to a lesser extend heterozygous) Neurod2-LacZ mice suffered from postnatal growth retardation, decreased motor performance and spontaneous seizures. All homozygous animals died between P14 and P35.

${ }^{3}$ Limb-clasping (also paw-clasping) is a pathological reflex that occurs in some genetic backgrounds and many mutant mice with lesions in the cerebral cortex, striatum, cerebellum or spinal cord (Lalonde and Strazielle 2011).
} 


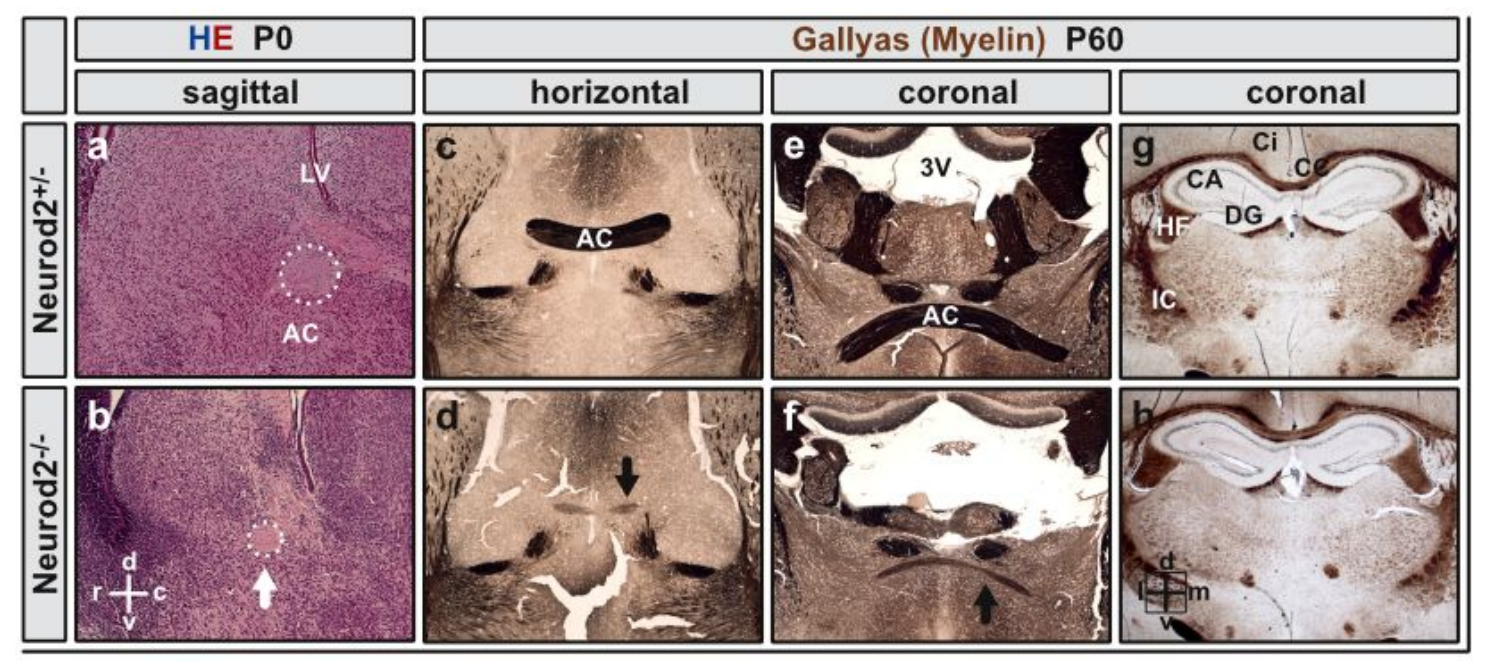

Figure 17: Hypoplastic anterior commissure in Neurod2-Null mice

Myelin staining by Gallyas silver impregnation in newborn (a,b) and 2 month old (c-h) heterozygous (a, c, e, g) and homozygous (b, $d, f, h)$ Neurod2-Null mice. $(\mathbf{a}, \mathbf{b})$ The diameter of the AC was clearly reduced at birth. (c-f) The AC was hypoplastic and hypomyelinated in adult Neurod2 deficient animals. (g, h) Formation and maintenance of the CC was not severely affected in homozygous Neurod2-Null mice. Arrows denote the AC in Neurod2 deficient brains. Dotted lines mark the cross sectioned AC in sagittal brain sections of newborn mice.

month. Homozygous Neurod2-Null mice that had survived this critical period had a normal life span, were fertile in both sexes, and females cared for their pups.

Postnatal death was initially observed in $~ 30 \%$ of homozygous Neurod2-Null mice. These animals were genetic hybrids resulting from blastocyst injection of R1 (129 hybrid) embryonic stem (ES) cells ${ }^{1}$ into C57BL/6 embryos. The lethality of homozygous Neurod2-Null mutants increased with continuous outbreeding into the C57BL/6 genetic background. This effect could be reversed by instead outbreeding into a 129X1/SvJ (129X1, formerly 129/SvJ) background. ${ }^{2}$ After four generations, almost all homozygous animals survived.

\subsubsection{Cortical Connectivity}

Gallyas silver impregnation (sect 4.7.4) of myelinated axon tracts revealed normal $\mathrm{CC}$ formation in adult Neurod2-Null mice (fig 17a-b; sect 1.5.2). The diameter of the AC, however, was strongly reduced in homozygous, but not in heterozygous animals at P60 (fig 17c-h). The calculated area of AC cross sections ${ }^{3}$ was reduced by more than $80 \%$ and the intensity of the myelin staining was decreased (arrows in fig $17 \mathrm{~d}, \mathrm{f}$ ). The AC cross section area was also smaller in sagittal sections of newborn Neurod2 deficient mice (dotted line in fig 17b).

\footnotetext{
${ }^{1}$ Threadgill et al. 1997: R1 embryonic stem cells are heterozygous for the 129/Sv (129S1, formerly $129 / \mathrm{Sv}$ P+Tyr+) and the 129cX/Sv (129X1, formerly 129/SvJ) alleles.

${ }^{2}$ A similar effect was reported for Neurod1-LacZ mice (sect 1.5.1 and Liu et al. 2000).

${ }^{3}$ The AC diameter was measured in coronal and horizontal brain sections and averaged. The area of the sagittal cross section was calculated assuming a circular shape by $A=\pi \cdot r^{2}$. Neurod2Null mice showed a reduction of approximately $85 \%$ when compared to wild type littermates.
} 
It is not clear whether the strongly reduced number of axons in the medial AC is due to neuronal loss or caused by the misguidance of commissural fibers.

\subsection{Simultaneous Inactivation of Neurod2/6}

$\mathrm{N}$ EUROD2/6 DOUBLE-DEFICIENT MICE were generated by crossbreeding the described Neurod2-Null and Neurod6-Cre mouse lines. ${ }^{1}$ Double homozygous offspring was born at the expected Mendelian ratio. The pups were initially not distinguishable from wild type littermates by eye. However, they did not feed and usually died within hours after birth.

The life span of Neurod2/6 double-deficient mice could be prolonged by outbreeding into the 129X1/SvJ genetic background (sect 2.3.1; sect 1.5.1). Surviving double-deficient animals showed severely retarded body growth, strong motor coordination deficits and seizures. Two double homozygous animals were hand fed and survived for almost two months (sect 2.4.3).

Most newborn Neurod2/6 double-deficient mice developed obvious breathing irregularities. Apnea periods frequently lasted several seconds and were usually followed by deep compensatory inspiration. Double homozygous animals became increasing cyanotic and eventually died.

Several neuron populations in the brainstem and in particular in the nucleus of the solitary tract (NST) express both, Neurod2 and Neurod6. ${ }^{2}$ The NST harbors the dorsal respiratory group, which controls respiratory timing and triggers inspiratory motor movements in a $\mathrm{pH}$ dependent manner. ${ }^{3}$ In mice deficient for the neuronal bHLH transcription factor Ascl1 (Mash1), the NST is atrophic and the differentiation of NST neurons is delayed, which can be rescued by overexpression of Neurod2. 4 The early lethality of most Neurod2/6 doubledeficient mice is presumably caused by primary central respiratory insufficiency due to mis-differentiation of brainstem neurons.

\footnotetext{
${ }^{1}$ Neurod6 deficient, Neurod2 heterozygous mice were fertile in both sexes, had a normal life span, and were routinely used to breed triple-deficient offspring. The average latency of successful mating was increased. In most cases, it took more than three days before freshly paired females received vaginal plugs. Females were not particularly aggressive towards approaching males. However, males appeared passive and not interested in sexual activity. Pregnant females were only moderately engaged in nest-building.

Data obtained from 19 virgin males that were each successfully mated to two virgin females resulted in first offspring after $25.7 \pm 1.3$ days (median 25). Assuming 19 days of pregnancy, fertilization must have happened within approximately 7 days (median 6). The birth frequency of permanent mating was $21.4 \pm 0.7$ days (median 21.8). Litters counted $7.0 \pm 0.2$ newborn pubs. The survival rate at weaning was $94.9 \%$. After weaning, offspring was $43.4 \%$ female. The genotype of all parent animals was Neurod $2^{\mathrm{Wt} / \mathrm{Null}} \times \mathrm{Neurod}^{\mathrm{Cre} / \mathrm{Cre}}$.

${ }^{2}$ Olson et al. 2001, fig 3b; Lin et al. 2004, fig 3L, tab 1; Goebbels et al. 2006, fig 3k, tab 1

${ }^{3}$ Feldman et al. 2003: The NST and various other brainstem nuclei are involved in the continuous sensing of $\mathrm{CO}_{2}$ and $\mathrm{pH}$, which underlies the control of breathing frequency. $\mathrm{CO}_{2}$ sensing was disrupted by experimental ablation of combinations, but not any single of these anatomical sites.

${ }^{4}$ Pattyn et al. 2006: Ascll is required for the generation of early (E10), but not of late born (E12) NST precursors. Expression of Neurog2 from the Mash1 promoter can rescue the generation of late born, but not of early born NST precursors. Considering this data, it is possible that NeuroD transcription factors (which are closely related to Neurog2, fig 7) control the differentiation of at least a subset of NST neurons.
} 


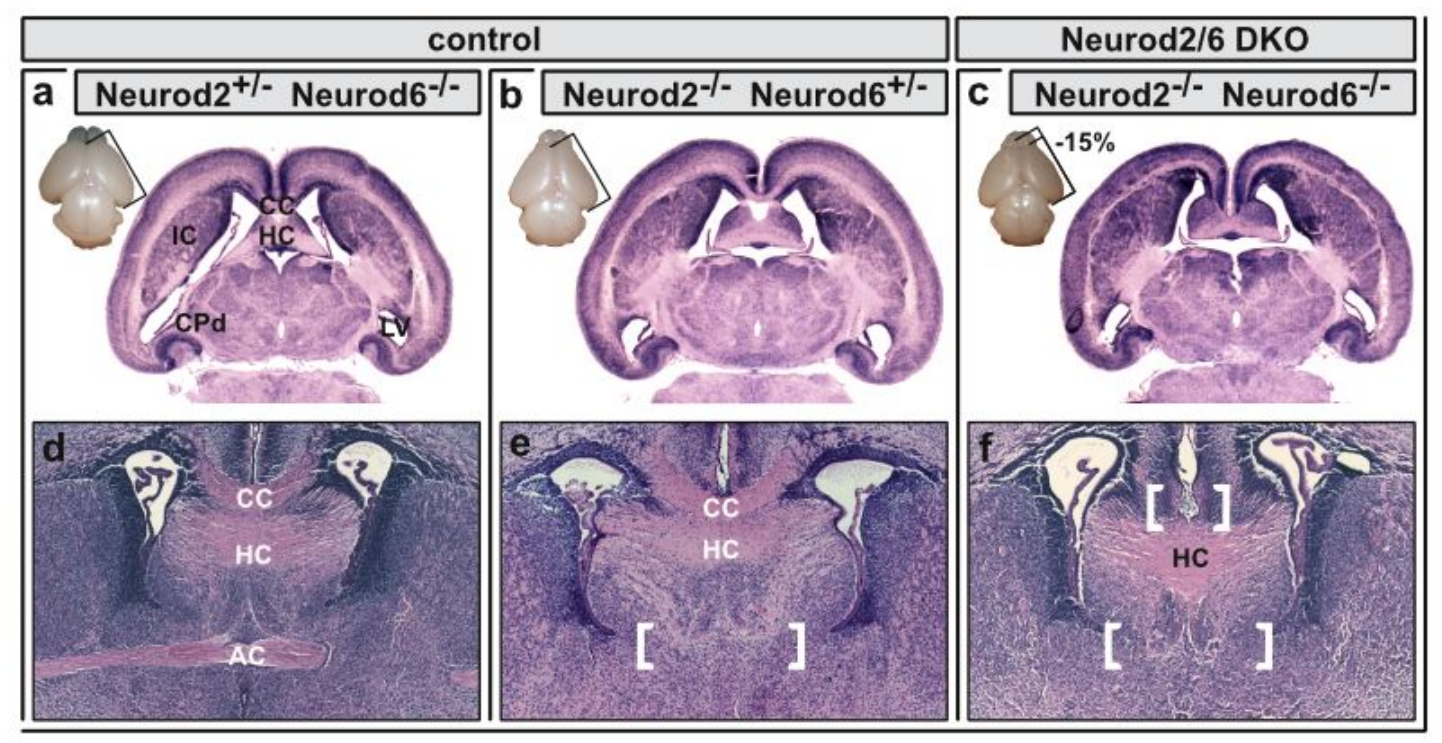

Figure 18: Brain anatomy of newborn Neurod2/6 double-deficient mice

Hematoxylin and eosin stained paraffin sections of newborn mice. (a, d) Neurod6 deficient mice with only one wild type copy of Neurod2 did not exhibit any obvious histological abnormality. (b,e) Vice versa, Neurod2 deficient mice with only on wild type copy of Neurod6 lacked the anterior commissure. (c, f) Neurod2/6 double mutants were additionally devoid of the corpus callosum. Apart from an irregularly stained cortical plate the general brain anatomy was not altered in double mutants. Brackets mark the absence of AC or CC. The upper panel $(\mathbf{a}-\mathbf{c})$ shows horizontal brain sections and whole brains in the upper left corners. The lower panel (d-f) shows coronal close-ups of the commissural plate.

The role of Neurod2/6 in breathing control is beyond the scope of this work and was not further studied. To prevent distress, offspring was used directly after birth or at late embryonic stages whenever possible.

\subsubsection{Brain Anatomy}

Macroscopically, brains of newborn Neurod2/6 double-deficient mice looked normal (inset in fig 18c), and the cerebral cortex was only mildly reduced in size. Histological examination confirmed that pyramidal neurons had been generated and had migrated into the $\mathrm{CP}$ and the hippocampus. $\mathrm{CC}$ and $\mathrm{AC}$, the two commissural fiber tracts originating from the neocortex, were entirely absent in double-deficient animals (fig 18f). Hematoxylin eosin (HE) stained serial sections of entire brains, did not provide evidence of aberrant axonal projections or Probst bundle (PB) formation (fig 19). Other prominent fiber tracts such as the internal capsule (IC), the hippocampal commissure (HC) and the hippocampal fimbria (HF) were not affected by the loss of Neurod2/6 (fig 18c, f; fig 19).

The CC was intact in mice harboring at least one wild type allele of Neurod2 or Neurod6 (fig 18d, e). The AC, however, was completely lost in animals with one wild type allele of Neurod6 in the absence of Neurod2 (fig 18e), but not vice versa (fig 18d). The AC did form in Neurod2 single-deficient mice with two wild type alleles of Neurod6, albeit at reduced size (fig 17). The latter suggests a gene dosage dependency on Neurod2 and to a lesser extent on Neurod6. 


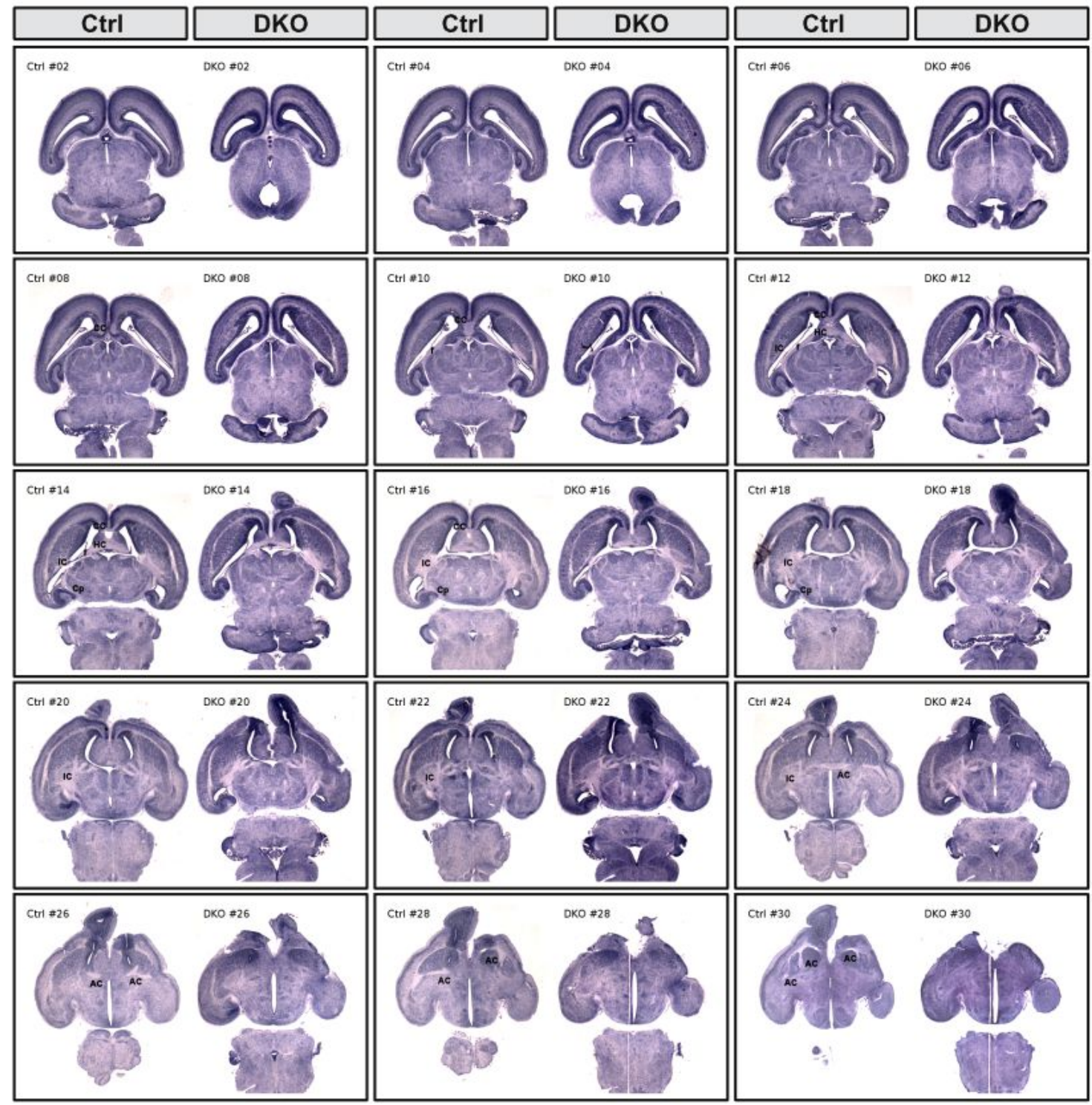

Figure 19: Absence of $A C$ and $C C$ in Neurod2/6 double-deficient mice

Series of HE stained horizontal brain sections of newborn control (Ctrl) and Neurod2/6 double-deficient (DKO) mice. There were no indications of $A C, C C$ or $P B$ formation in any section of five equally processed Neurod2/6 double-deficient brains. For space constrains, only 15 sections are shown here. The whole data set is available as annotated movie and as registered three dimensional image stacks upon request.

\subsubsection{Cortex Development}

So far, complete agenesis of $\mathrm{CC}$ and $\mathrm{AC}$ were the most prominent features observed in Neurod2/6 double-deficient animals. The obvious question was whether the subset of pyramidal neurons that normally project callosally did exist, or whether those cells had died from apoptosis, as previously described for hippocampal granule cells in Neurod 1 deficient mice. ${ }^{1}$ Brain sections from

\footnotetext{
${ }^{1}$ Miyata et al. 1999: Neurod1 deficient mice that had been rescued from early postnatal death using an insulin dependent Neurod 1 expression cassette showed increased apoptosis and loss of most granule cells in the hippocampal dentate gyrus at P6 and P30 (fig 5e, f); Liu et al.
} 


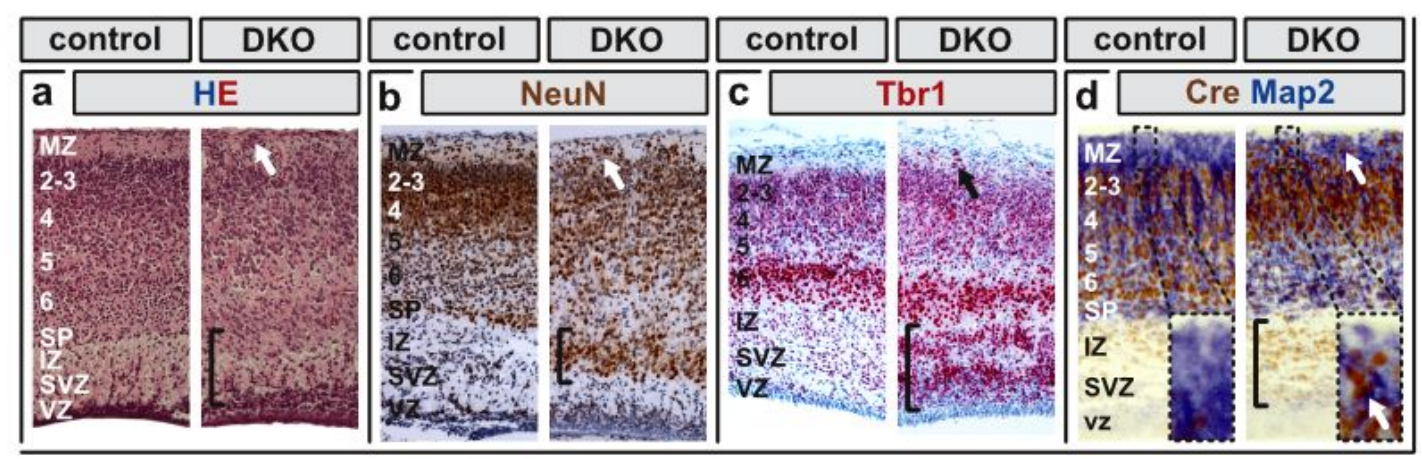

Figure 20: $\mathrm{CP}$ formation in newborn Neurod2/6 double-deficient mice

Paraffin sections of newborn control and Neurod2/6 double-deficient (DKO) mice. (a) HE staining shows ectopic accumulations of cells in the SVZ, IZ and MZ of double-deficient animals. (b) IHC for the panneuronally expressed protein NeuN (brown) identified the accumulated cells in the IZ and $M Z$ as committed neurons. (c) IHC for the neuronal determination and differentiation factor Tbr1 (red) suggested that the cellular accumulations in the SVZ may comprise uncommitted neuronal precursors. (d) Double IHC for Cre (brown) and Map2 (blue) confirmed that the cells ectopically localized in the IZ were immature and did not identify as Map2-positive bipolar pyramidal neurons. Despite the complete absence of Neurod2 and Neurod6 gene functions, the Neurod6 promoter was active at $P$ 0. Arrows point to ectopically localized pyramidal neurons in the MZ. Brackets denote cell accumulations in the SVZ and IZ.

newborn Neurod2/6 double-deficient animals were probed for various proteins that are normally specifically expressed in certain cell types or cortex layers ${ }^{1}$ (sect 1.2.4).

\subsubsection{Overview}

HE staining confirmed normal thickness of the neocortex and showed approximately normal overall cell numbers in the CP of newborn Neurod2/6 doubledeficient mice (fig 20a). SVZ, IZ and MZ normally harbor relatively few cells. In double-deficient animals, however, the cellular density was increased in these layers (arrows and brackets in fig 20). Immunohistochemistry (IHC) for neuronal nuclear antigen (NeuN), a protein expressed by most committed neurons, ${ }^{2}$ confirmed that pyramidal cells were able to differentiate and migrate radially into the CP despite the absence of Neurod2/6 (fig 20b). NeuN was strongly expressed by cells that had accumulated in the Neurod2/6 double-deficient SVZ/IZ (bracket in fig 20b).

$\mathrm{T}$ (Brachyury) brain 1 (Tbr 1 ) is a transcription factor involved in the determination and differentiation of cortical pyramidal neurons. ${ }^{3}$ During normal embryonic

2000: Neurod1 deficient mice that had been bread to the Sv129X1/SvJ genetic background to overcome early postnatal death (sect 1.5.1) showed increased levels of apoptosis and loss of most dentate gyrus granule neurons at P11 (fig 4g, h); Schwab et al. 2000: Neurod 1/6 doubledeficient mice showed normal levels of mitosis but strongly $(\sim 7$ fold $)$ increased apoptosis in the dentate gyrus region at P2 (fig 4).

${ }^{1}$ Molyneaux et al. 2007: Comparison of a large number of cell type-specifically expressed marker genes in the developing cortex

${ }^{2}$ Mullen et al. 1992; Sarnat et al. 1998

${ }^{3}$ Bulfone et al. 1995; Hevner et al. 2001; Englund et al. 2005; Arnold et al. 2008 
development, Tbr 1 is expressed in the SVZ, IZ and layer 1-5 of the CP at moderate levels. Corticothalamically projecting pyramidal neurons in layer 6 normally expresses Tbr 1 at much higher levels. ${ }^{1}$ In principle, these patterns were maintained in newborn Neurod2/6 double-deficient mice. SVZ, IZ and MZ, however, were submerged with strongly Tbrl expressing cells (fig 20c). Hypothetically, these might be undifferentiated SVZ-progenitors, or basically differentiated multipolar neurons that had failed terminal differentiation, or differentiated neurons of partial deeper-layer identity.

IHC for Cre recombinase, which in these mice is expressed under control of the endogenous Neurod6 promoter, was used to visualize Neurod6 promoter activity in the absence of Neurod6 transcription. Cre signals resemble putative Neurod6 expression patterns. In Neurod2/6 double-deficient mice, Neurod6 promoter activity was increased in upper cortical layers while it was decreased in deeper layers (fig 20d). Cre was expressed at relatively high levels by neurons ectopically located in the $\mathrm{MZ}$ to (arrows in fig 20d), and at relatively low levels by cells that had accumulated in the SVZ and IZ (brackets in fig 20d).

Microtubule associated protein 2 (Map2) is another frequently used pan-neuronal marker protein. The Map2a+2b antibody recognizes two isoforms that are targeted to the dendritic compartment and that are specifically expressed by terminally differentiated neurons. ${ }^{2}$ Immunohistochemical Map2a2b signals were confined to the CP in newborn controls and Neurod2/6 double-deficient mice (fig 20d). NeuN-positive cells that had accumulated the SVZ and IZ failed to express Map2 or other markers that are normally CP specifically expressed (fig 22; fig 23). This suggests that in newborn Neurod $2 / 6$ double-deficient mice, a subset of determined pyramidal neurons had failed to differentiate terminally and subsequently did not migrate radially into the CP. Neurod6-Cre positive / NeuN-positive / Tbr1-positive / Map2-negative cells ectopically localized in the IZ are thus most probably multipolar pyramidal neurons that had failed to undergo multipolar-to-bipolar transition ${ }^{3}$ (sect 1.2.3).

\subsubsection{Radial Migration}

Neocortical pyramidal neurons are born in the VZ between E12 and E17. They normally migrate radially into the $\mathrm{CP}$, but never reach the outer surface of the brain. ${ }^{4}$ Regardless of the neuron's birth date, radial migration stops abruptly as soon as the cell body approaches the MZ, a thin sheet of Cajal-Retzius cells covering the surface of the entire CP. ${ }^{5}$ Cajal-Retzius cells secrete the glycoprotein

\footnotetext{
${ }^{1}$ Han et al. 2011; McKenna et al. 2011: High Tbr1 expression in layer 6 is sufficient for the repression of Fezf2 and necessary for the formation of corticothalamic connectivity.

${ }^{2}$ Izant and McIntosh 1980; Cassimeris and Spittle 2001; Dehmelt and Halpain 2005; OhtakaMaruyama et al. 2013

${ }^{3}$ Ohtaka-Maruyama et al. 2013, fig 3D, E: A similar phenotype was observed in mice that were deficient for the transcription factor RP58, an effector of Neurog2.

${ }^{4}$ Lipnick and Jacobson 2006, chapter 8 by Frank Polleux and E. S. Anton, sect "Termination of Migration" (p 229f): "Once neurons reach the top of the CP, the movement of neurons stops abruptly at the interface between the CP and the [...] marginal zone [...]. This final stage of neuronal migration is the least explored aspect of neuronal migration, in spite of its significance for genetic and acquired cortical malformations."

${ }^{5}$ Villar-Cerviño et al. 2013: "CR cells cover the entire cortical surface before the emergence of the cortical plate"
} 


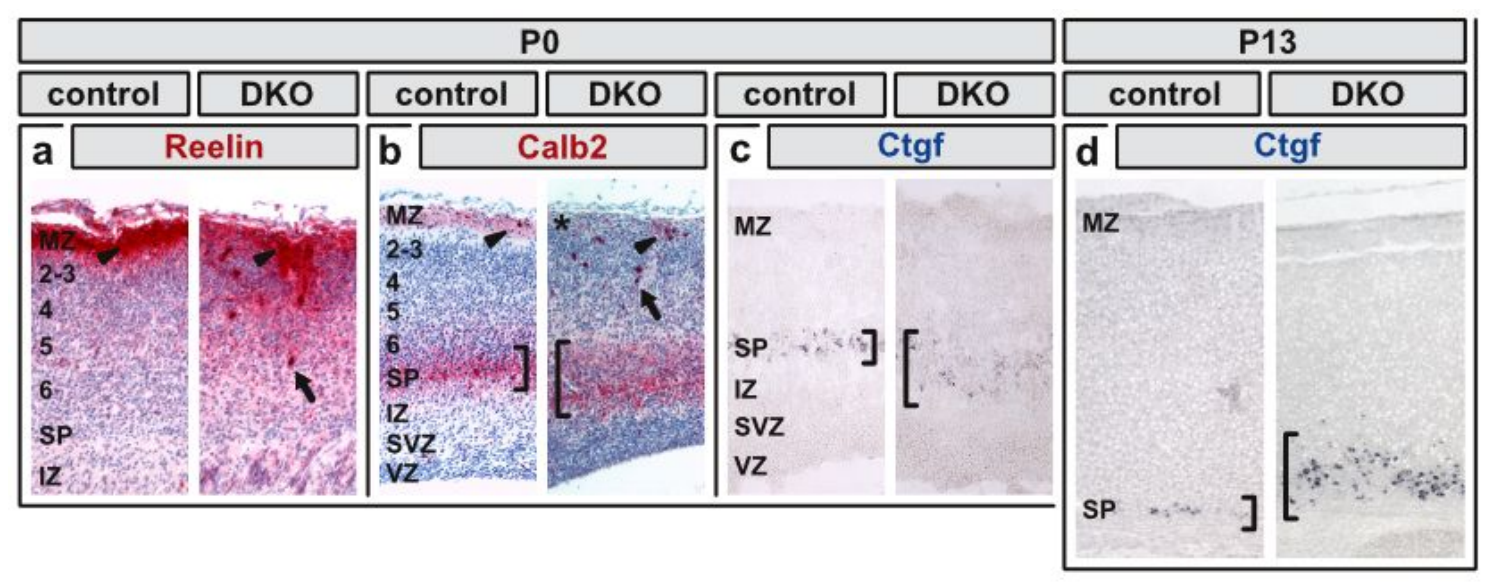

Figure 21: Marginal zone and subplate in Neurod2/6 double-deficient mice

(a, b) Highly sensitive IHC in horizontal paraffin sections from newborn mice. Primary antibodies were detected using AP and FastRed (red with blue counter stain). Reelin (a) marks the MZ and identifies individual Cajal-Retzius cells. Calb2 (b) marks the SP and a subset of Cajal-Retzius cells. (c-d) In situ hybridization for Ctgf (blue) on coronal frozen sections from newborn (c) and two week old mice (d). The SP is dispersed and contains more cells in Neurod2/6 double-deficient brains. Arrows point to ectopically located Cajal-Retzius cells in the CP. Arrow heads denote Cajal-Retzius cells in the MZ. Brackets mark the SP.

Reelin and are discussed to mediate cellular attraction towards the brain surface. They are also thought to induce the detachment of migrating pyramidal neurons from radial glia cells, thereby terminating radial migration ${ }^{1}$ (sect 1.2 .3 ).

In Neurod2/6 double-deficient mice, increased cell densities below and above the $\mathrm{CP}$ suggested defects in the initiation and termination of radial migration. Disjoint subsets of pyramidal neurons accumulated in the SVZ/IZ, or over-migrated into the MZ (fig 20a). IHC for Cre recombinase confirmed that many cells in the MZ of Neurod2/6 double-deficient brains were Neurod6-positive pyramidal neurons (fig 20d). Strong Brn2, Lmo4 and Satb2 expression (arrows in fig 22) and the absence of Foxp2 and Sox5 (fig 23) suggested an upper-layer identity.

Signaling from the MZ was not expected to be affected because Cajal-Retzius cells never express Neurod $2^{2}$ or Neurod $6^{3}$ (fig 13c). IHC for Reelin and calbindin 2 (Calb2) confirmed the presence of functional Cajal-Retzius cells in the MZ of newborn Neurod2/6 double-deficient mice (arrow heads in fig 21a,b). Reelin staining was not significantly reduced in the $M Z$, but diffusely increased in layer 2-6. The CP of double-deficient animals was scattered with strongly Reelinand Calb2-positive cells (arrows in fig $21 \mathrm{a}, \mathrm{b}$ ). These were probably regular CajalRetzius cells that had been bypassed by a large number of radially migrating pyramidal neurons and that had been thereby incorporated into the expanding

\footnotetext{
${ }^{1}$ Tissir and Goffinet 2003

${ }^{2}$ Olson et al. 2001, fig 3F; Lin et al. 2005, fig 4J; Ince-Dunn et al. 2006, fig 2B, D, F Note: Lin et al. 2005, tab 1 states that lacZ expression was detectable in all "cerebral cortex layers" which should probably be interpreted as "all layers of the" because the MZ is clearly negative in all provided images.

${ }^{3}$ Goebbels 2002, fig 7G, 8A, D; Goebbels et al. 2006, fig 2i,j, 3d, tab 1
} 
CP. ${ }^{1}$ The predominant localization of Reelin-positive cells in the upper $\mathrm{CP}$ is consistent with the assumption that over-migrating pyramidal neurons originate from the pool of upper layer neurons (arrows fig $21 \mathrm{a}, \mathrm{b}$ ).

The fact that a minority of Neurod2/6 double-deficient pyramidal neurons overmigrated into the $\mathrm{MZ}$ while the majority correctly terminated radial migration suggests substantial heterogeneity in the control of radial migration and neuronal detachment. Signaling from Cajal-Retzius cells is not only involved in the termination of radial migration, but also in the multipolar-to-bipolar transition, which is a prerequisite for the (re-)initiation of radial migration in the $I Z .^{2}$ The failure of another minority of Neurod2/6 double-deficient pyramidal cells to migrate from the SVZ/IZ into the CP might thus be explained by a similar mechanism at an earlier differentiation stage.

\subsubsection{Subplate}

The neocortical SP originates from the first radially migrating neurons, which are born at E10.5 in the VZ. It can be regarded as deepest cortex layer, and it is sometimes referred to as layer $6 b$ or 7 . During corticogenesis, SP cells temporarily interact with afferent thalamocortical axons. ${ }^{3}$ The SP is discussed to propagate the early protomap, a blueprint for neocortical arealization and thalamocortical connectivity. ${ }^{4}$ After birth, most SP cells transform into white matter interstitial cells, layer 6 pyramidal neurons, or undergo apoptosis. ${ }^{5}$

As shown by IHC for Calb2 (fig $21 \mathrm{~b}$ ) and by ISH for connective tissue growth factor (Ctgf) (fig 21c), the SP was broadened and SP cells were dispersed in newborn Neurod2/6 double-deficient mice. Normally, most SP cells disappear during the first two postnatal weeks, which results in a thin band of Ctgf expressing cells at P13. ${ }^{6}$ In the absence of Neurod2/6, however, the number of Ctgf-positive cells was increased and the SP layer was more prominent at P13 than at birth (fig 21d).

\footnotetext{
${ }^{1}$ Hypothetical mechanism: Initially, some Cajal-Retzius cells would be bypassed by a few pyramidal neurons that became insensitive to the repulsive detachment signal. Once physically separated from the MZ, these isolated Cajal-Retzius cells would not be capable to stop radial migration of other pyramidal neurons. Generation after generation would pass the lagged Cajal-Retzius cells and lead to their assimilation into the thickening CP.

${ }^{2}$ Jossin et al. 2003: Pyramidal neuron precursors are born in the VZ and migrate radially into the IZ where they settle temporarily, polarize, transition from multipolarity to bipolarity, and then continue to migrate further into the CP. Reelin signaling regulates the expression of Rap 1 and Cdh2 (fig 5a,b,e), which are required for the vertical orientation and radial migration pyramidal cells in the SVZ (fig 1-5). Overexpression of a dominant negative variant of the Reelin-receptor VLDLR abolishes vertical orientation and radial migration into the CP (fig 5c). That latter can be rescued by co-electroporation of the Reelin-effectors Rat 1 or AKT.

${ }^{3}$ Ghosh and Shatz 1993: Experimental SP ablation by injection of kainic acid in cats prevented afferent thalamic axons to enter the $\mathrm{CP}$

${ }^{4}$ O'Leary et al. 2007b

${ }^{5}$ Okhotin and Kalinichenko 2003

${ }^{6}$ Heuer et al. 2003, fig 3f-h
} 


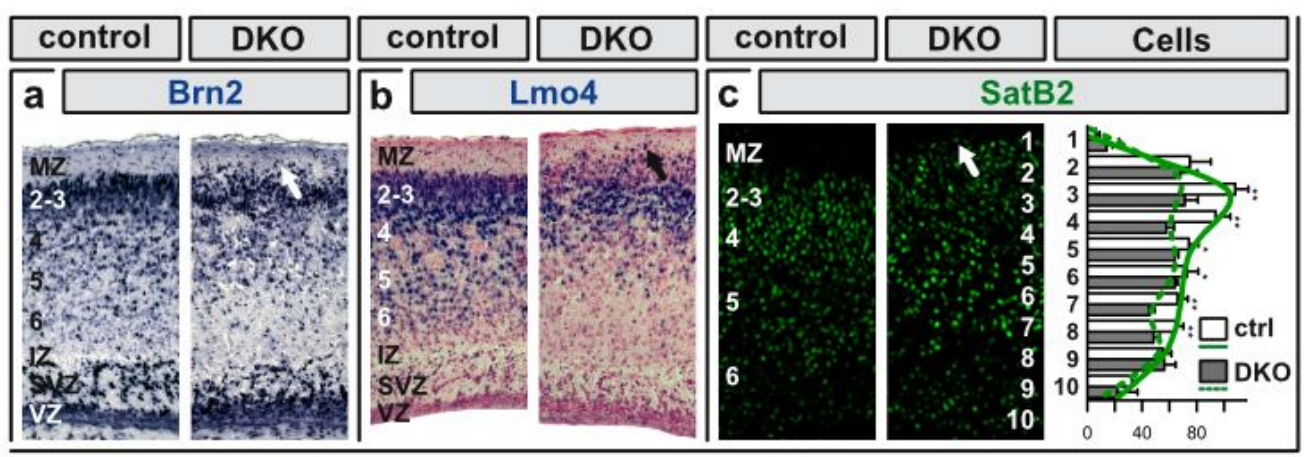

Figure 22: Upper cortex layers in Neurod2/6 double-deficient mice

Paraffin sections of newborn mice. Immunohistochemistry for (a) Brn2 (blue), (b) Lmo4 (blue with red counter stain) (c) Satb2 (green). The graph visualizes the relative radial distribution of Satb2-positive cells in the cortical plates of newborn control (white bars, solid line) and Neurod2/6 double-deficient (gray bars, dashed line) mice. Fluorescent co-immunostaining for Satb2, Ctip2, Sox5 and DAPI was performed on horizontal brain sections from nine littermates that were always processed together and embedded in one paraffin block. In color merged digital images, the cortical plate was split into ten equally sized bins (1-10). Positive cells were manually counted by a double-blinded colleague. The bar plot visualizes the average cell number per bin; whiskers show the SEM; $p$-values are displayed as ${ }^{*} p<0.05,{ }^{* *} p<0.0 .1,{ }^{* * *} p<0.001$; regression curves were fitted over 50 equally sized bins. The total number of Satb2-positive cells was reduced from 673 ( $\pm 60, n=5$ Neurod $2^{+/-} \times$Neurod $\left.6^{-/-}\right)$to $520\left( \pm 40, n=4\right.$ Neurod $2^{-/-} \times$Neurod6 $\left.6^{-/-}\right)$cells per equally sized radial unit $(p-v a l u e=0.074)$. Arrows point to ectopically located cells in the MZ. The Lmo4 expression pattern varies greatly between cortical areas (sect 2.4.6).

\subsubsection{Upper Layers}

Pyramidal neurons located in upper neocortical layers, project to the ipsilateral or contralateral hemisphere and contribute most axons of the corpus callosum. ${ }^{1}$ Neurod2/6 double-deficient mice entirely lack a CC, raising the questions (i) whether the neurons that normally project callosally survived, (ii) if they had migrated into the upper CP, and (iii) whether upper layer neurons were molecularly defined as such. Immunohistochemistry was performed for several proteins that are known to be selectively expressed in upper layers or by callosal projection neurons.

brain 1 (Brn1) and brain 2 (Brn2) are closely related class III POU domain transcription factors predominantly expressed in the VZ, the SVZ, and upper layers of the cortical plate. ${ }^{2}$ Brn $1 / 2$ are regarded as early determinants of the upper layer neuron fate, and are essential for Reelin signaling, radial migration and the specification of upper layer neurons. ${ }^{3}$ In Neurod2/6 double-deficient animals, the level of Brn2 expression was not severely altered (fig 22a). This suggests that the determination and the early differentiation of upper layer neurons do not critically depend on Neurod $2 / 6$.

\footnotetext{
${ }^{1}$ Fishell and Hanashima 2008, fig 1: Summary of the transcriptional control of neocortical projection tracts formation

${ }^{2}$ McEvilly et al. 2002, fig 1: Brn1 and Brn2 are co-expressed in superficial and ventricular layers of the mouse cerebral cortex at PO.

${ }^{3}$ McEvilly et al. 2002; Sugitani et al. 2002
} 
Lim domain only 4 (Lmo4), is a transcription factor involved in cortical arealization (sect 2.4.6). Outside of the somatosensory cortex, it is predominantly expressed in upper layers and by callosally projecting neurons located in layer $5 .{ }^{1} \mathrm{Lmo} 4$ expression is downregulated in Satb2 deficient mice. ${ }^{2}$ In Neurod2/6 doubledeficient mice Lmo4 was expressed in upper cortical layers (fig 22c) of most cortical areas including the somatosensory cortex (fig 31). This suggests that Neurod2/6 double-deficient pyramidal neurons in the upper neocortical layers did differentiate to a degree where they had acquired a basic callosal identity. The abnormal persistence of Lmo4 expression in the area of the somatosensory cortex, however, points towards disturbed cortical arealization (sect 3.4).

Special AT-rich sequence binding protein 2 (Satb2) is a transcriptional repressor expressed by most callosally projecting pyramidal neurons. ${ }^{3}$ Satb2 is normally enriched in upper neocortical layers. ${ }^{4}$ It directly suppresses Ctip2, a subcortical determinant enriched in deeper layers (fig 23a), and thereby regulates callosal versus subcortical axon growth ${ }^{5}$ (sect 2.4 .2 .5 ). In Satb2 deficient mice, upper layer neurons fail to project via the $\mathrm{CC}$ and instead grow their axon into the AC and the corticospinal tract (CST). ${ }^{6}$ In newborn Neurod $2 / 6$ double-deficient mice, Satb2 was expressed (fig 22b) but the total number of Satb2 expressing cells was reduced by a fourth $(23 \%)$. The remaining Satb2-positive pyramidal neurons were more evenly distributed along the radial axis of the CP (graph in fig 22b).

Taken together, number and molecular fate of upper layer pyramidal neurons were moderately changed upon simultaneous inactivation of Neurod2 and Neurod6. At birth, most pyramidal neurons had migrated radially into the cortical plate. Neurons with molecular upper layer identity had correctly bypassed the deeper layers, although some of them had migrated too far and were located in the MZ. The observed abnormalities are too mild to solely explain the total absence of the CC in Neurod2/6 double-deficient mice.

\subsubsection{Deeper Layers}

COUP-TF interacting 2 (Ctip2) is enriched in deep layers (5 and 6) of the neocortex. Ctip2 is essential for fasciculation and pathfinding of CST axons. ${ }^{7}$ In upper cortical layers, Ctip2 expression is actively repressed by Satb2. ${ }^{8}$ Overexpression of Ctip2 in upper layer neurons is sufficient to change axon trajectories from

\footnotetext{
${ }^{1}$ Huang et al. 2009; Azim et al. 2009

${ }^{2}$ Alcamo et al. 2008 Lmo4 expression is reduced in the dorsomedial cortex of Satb2 deficient mice (tab 1; fig 6).

${ }^{3}$ Britanova et al. 2008; Alcamo et al. 2008; Leone et al. 2008

${ }^{4}$ Britanova et al. 2005: "By E18.5 the majority of Satb2-positive cells occupy the upper part of the CP”

${ }^{5}$ Britanova et al. 2008, fig 4: Satb2 deficient mice lack a corpus callosum and the proportion of upper layer neurons back-labeled by DiI injections into the AC and the IC is increased.

${ }^{6}$ Alcamo et al. 2008, fig 3: Satb2-LacZ mice demonstrate that Satb2 promoter-positive neurons project to IC, EC and CPd in the absence (but never in the presence) of Satb2.

${ }^{7}$ Arlotta et al. 2005, fig 7: Cortical pyramidal neurons fail to extend their axons to spinal cord in Ctip2 deficient mice.

${ }^{8}$ Britanova et al. 2008, fig 8e; Baranek et al. 2012, fig $5 i$
} 


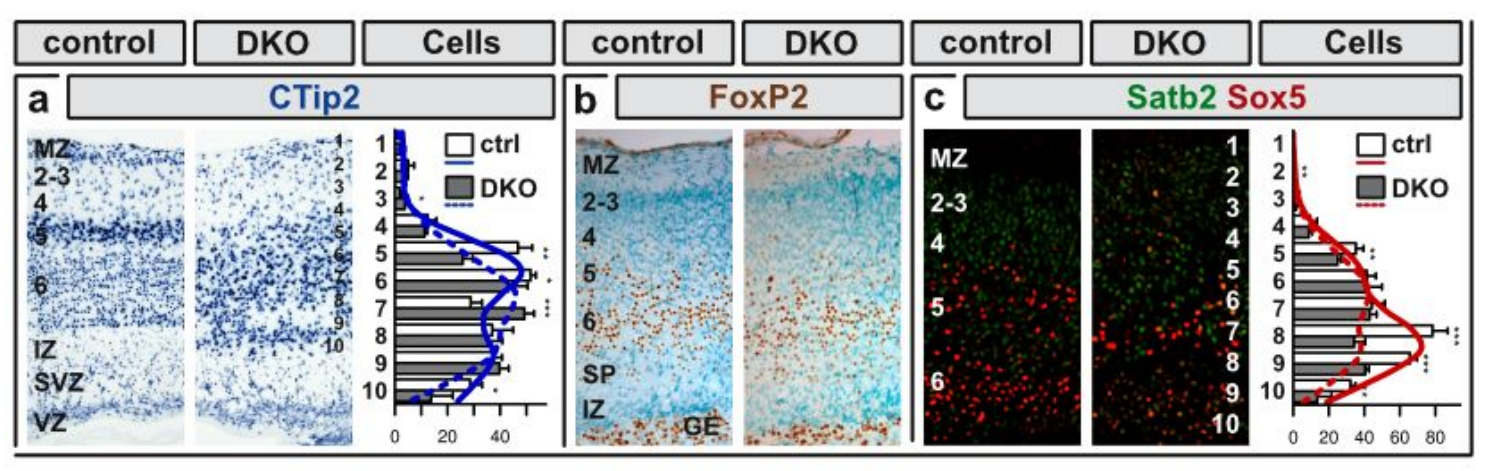

Figure 23: Deeper cortex layers in Neurod2/6 double-deficient mice

Paraffin sections of newborn mice. Immunohistochemistry for (a) Ctip2 (blue), (b) Foxp2 (brown with blue pseudo-colored counter stain), and (c) Sox5 (red with Satb2 in dark green). The quantification of Ctip2- and Sox5-positive cells $(\mathrm{a}, \mathrm{c})$ were done in the same way and on the same co-stained tissue sections as those of Satb2-positive cells in fig 22c.

callosal to subcortical targets. ${ }^{1}$ In accordance with relatively normal Satb2 expression in upper layers (fig 22b), Ctip2 expression was not significantly altered in Neurod2/6 double-deficient mice (fig 23a). The total number of Ctip2positive neurons was reduced by less than $10 \%$ (graph in fig 23a). However, the segregation of larger and smaller Ctip2-positive pyramidal neurons to layer 5 and layer 6, respectively, was lost (fig 23a).

Forkhead box P2 (Foxp2) is a transcription factor specifically expressed in cortex layer 6. ${ }^{2}$ Foxp2 expression and the positioning of Foxp2-positive neurons in layer 6 were not significantly altered in Neurod2/6 double-deficient mice (fig 23b).

Ctip2 is a major downstream effector of Fez family zinc finger 2 (Fezf2). ${ }^{3}$ Expression levels of Ctip2 and Foxp2 are strongly reduced in Fezf2 deficient mice. ${ }^{4}$ In regard of normal Ctip2 and Foxp2 expression patterns in Neurod2/6 doubledeficient mice, it can be assumed that Fezf2 expression is not lost in absence of Neurod2/6.

SRY-box 5 (Sox5) is a transcriptional repressor preferentially expressed in deeper cortical layers. It is thought to control the timing of subcortical projection neuron differentiation by inhibiting genes that promote the subcortical fate. ${ }^{5}$ Sox5 expression is essential for targeted growth of corticothalamic and corticospinal axons. ${ }^{6}$ In Neurod2/6 deficient mice, Sox5 was expressed at lower levels in deeper layer neurons (fig 23c) and the total number of Sox5-positive cells was

\footnotetext{
${ }^{1}$ Chen et al. 2008, fig 5: In utero electroporation of Fezf2 or Eomes cDNA into the developing cerebral cortex results in upper layer neurons projecting to the cerebral peduncle, thalamus and pontine nucleus.

${ }^{2}$ Ferland et al. 2003, fig 2, tab. 1; Takahashi et al. 2008, fig 3b

${ }^{3}$ Chen et al. 2008: Ctip2 is downregulated in Fezf2 deficient mice (fig 3), which fail to form subcerebral projections to the spinal cord. Ctip2 overexpression in neurons of the Fezf2 deficient cortex can rescue the formation of subcerebral projections (fig 4,5) but Fezf2 overexpression is not sufficient to induce Ctip2 the same animals (fig S3-S5).

${ }^{4}$ Molyneaux et al. 2005, fig 5h; Chen et al. 2005, tab 1, fig 1

${ }^{5}$ Lai et al. 2008

${ }^{6}$ Kwan et al. 2008
} 


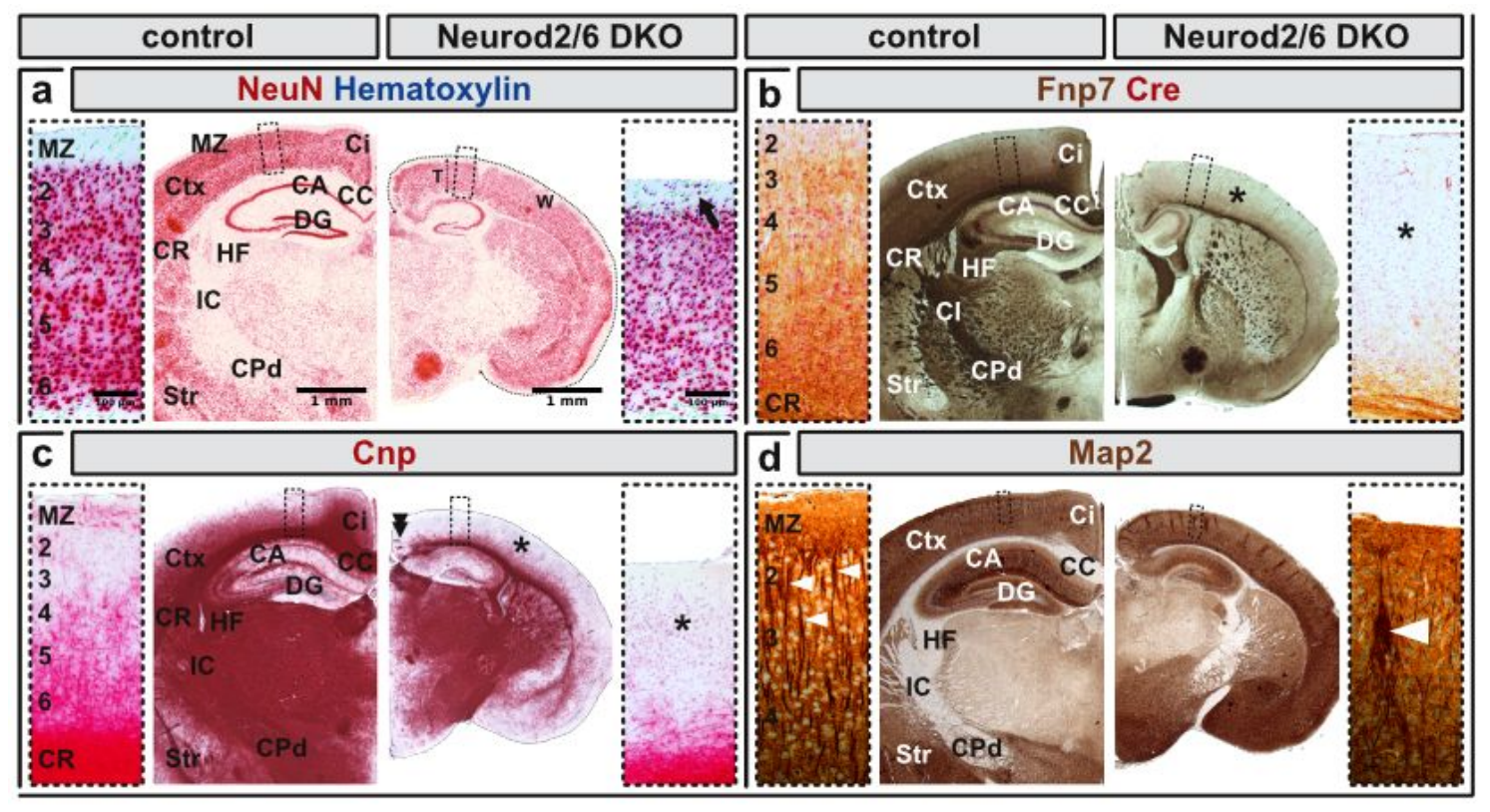

Figure 24: Brain anatomy of adult Neurod2/6 double-deficient mice

Paraffin sections of two months old control and Neurod2/6 double-deficient mice. IHC for (a) the pan-neuronal marker NeuN (red) and hematoxylin counter staining (blue), (b) axonally localized non-phosphorylated neurofilaments (Fnp7) (brown) and Cre to visualize Neurod6 promoter activity (red), (c) the myelin protein Cnp (red), and (b) the dendritic marker Map2 (brown). Coronal brain sections from control and Neurod2/6 doubledeficient mice are shown at the same scale. Close-ups were taken approximately from the boxed regions. The arrow points to ectopically localized pyramidal neurons in the MZ. Asterisks mark virtual absence of Fnp7 and Cnp. The double arrow head points to callosal axons terminating in the ipsilateral Ci. Single arrow heads mark bundles of apical pyramidal neuron dendrites that are abnormally enlarged in Neurod2/6 double-deficient animals. Blue (1) and green (3) marks in (a) denote manually counted NeuN-positive and -negative cells, respectively.

reduced by one third (32\%) in horizontal brain sections of newborn Neurod2/6 double-deficient mice (graph in fig 23c).

\subsubsection{Adult Brain}

Sporadically, Neurond2/6 double-deficient mice survived for a prolonged period after birth (sect 2.4). They developed spastic motor dysfunction with choreatiform trunk and limb movements. Just before weaning at P 21, the body weight was reduced by more than two thirds (eg Neurod2/6 double-deficient male $3.3 \mathrm{~g}$ in comparison to Neurod2 ${ }^{\mathrm{Wt} / \mathrm{Null}} \times$ Neurod6 ${ }^{\mathrm{Cre} / \mathrm{Cre}}$ male control littermate $10 \mathrm{~g}$, which was already lighter than age matched wild type males). Naturally, surviving Neurod2/6 double-deficient animals died latest at the time of weaning. A similar effect had been reported for Neurod2 single-deficient mice, which developed hypothalamic insufficiency and cretinism. ${ }^{1}$

Some Neurod2/6 double-deficient pups were offered regular hand-feeding with water and a sugared milk powder solution. Those that accepted the supple-

\footnotetext{
${ }^{1}$ Lin et al. 2006: Neurod2 is expressed in the hypothalamus and pituitary gland neurons. Neurod2 deficient mice suffered from typical signs of cretinism and showed reduced TSH and T4 levels.
} 
mentary food slowly gained weight. However, they never exceeded 6 grams and spontaneously died at the age of five to eight weeks for unknown reason.

Two hand-fed Neurod2/6 double-deficient mice survived for nearly two months. One could be retrocardially perfused at P60 and was used for the quantitative analysis of paraffin embedded brain sections. The thickness of the neocortex was decreased by $16 \%(\mathrm{~T}=630 \mu \mathrm{m}$ vs. $530 \mu \mathrm{m}$ in fig $24 \mathrm{a})$. The mediolateral width of the neocortical surface was reduced by $29 \%(\mathrm{~W}=10.5 \mathrm{~mm}$ vs. $7.5 \mathrm{~mm}$ in fig 24a). The deduced neocortical volume was reduced by $57 \%{ }^{1}$

The number of neurons per tangential unit was not decreased $\left(\mathrm{C}^{+}=598\right.$ vs. 596 NeuN-positive cells; $\mathrm{C}^{-}=130$ vs. 150 NeuN-negative cells; and $\mathrm{C}^{\sum}=728$ vs. 746 total cells per $250 \mu \mathrm{m}$ in fig 24a). The latter in combination with the decreased thickness results in an increase of neocortical cell density $\left(\mathrm{D}^{+}=3797\right.$ vs. 4498 NeuN-positive cells $/ \mathrm{mm}^{2}=+18 \%$; $\mathrm{D}^{-}=825$ vs. $1132 \mathrm{NeuN}$-negative cells $/ \mathrm{mm}^{2}$ $=+37 \%$; and $\mathrm{D}^{\sum}=4622$ vs. 5630 total cells $/ \mathrm{mm}^{2}=+22 \%$ ). Based on these calculations, the approximated total number of cells in the Neurod2/6 doubledeficient neocortex was reduced by $44 \% .^{2}$

Neurofilament staining revealed strongly reduced neocortical axonal connectivity (fig 24b). The latter was accompanied by a proportional degree of hypomyelination (fig 24c). The density of the dendritic compartment was not reduced per se, but Map2-positive bundles of apical dendrites were massively enlarged (arrow heads in fig 24d). Radial dendritic bundles (dendritic minicolumns) normally contain only few apical dendrites originating from large pyramidal neurons in layer 5 that are accompanied by apical dendrites originating from smaller neurons in layer 2 and $3 .^{3}$

\subsubsection{Cortical Connectivity}

\subsubsection{Callosal Projections}

Direct stereotypic interconnection of the two cerebral hemispheres via the CC is a complex task achieved during later brain development. The path taken by growing callosal axons can be divided into two symmetrical stages. During the first stage, they turn medial in the IZ, fasciculate to form a compact tract, grow towards the midline, and cross to the contralateral hemisphere. The second stage

\footnotetext{
${ }^{1}$ The neocortical surface area was assumed as being circular and determined by the mediolateral neocortical width $\mathrm{W}$. The neocortical thickness T was assumed to be uniform per animal. The total neocortical volume $\mathrm{V}$ was calculated as $\mathrm{V}=0.25 \cdot \mathrm{W}^{2} \cdot \pi \cdot \mathrm{T}$. For the brains shown in fig $24 \mathrm{a}$, $\mathrm{V}=54.6 \mathrm{~mm}^{3}$ in the control and $\mathrm{V}=23.4 \mathrm{~mm}^{3}$ in the Neurod2 $/ 6$ double-deficient animal which results in a volume reduction of $57 \%$.

${ }^{2}$ Total neocortical cell numbers $\mathrm{N}$ were roughly estimated from the calculated volume $\mathrm{V}$ and the cellular density $\mathrm{D}$ as $\mathrm{N}=\mathrm{V} \cdot \mathrm{D} \cdot 100$ (brain tissue had been sectioned at $10 \mu \mathrm{m}$, which resulted in 100 sections per $\mathrm{mm}$ ). $\mathrm{N}^{+}=20.73$ vs. 10.53 million NeuN-positive cells $=-49 \% ; \mathrm{N}^{-}=4.5$ vs. 2.65 million NeuN-negative cells $=-41 \% ; \mathrm{N}^{\sum}=25.2$ vs. 13.2 million total cells $=-48 \%$

${ }^{3}$ Dendritic bundles (or dendritic minicolumns) were described in different neocortical areas of many mammalian species. A detailed study in the macaque brain quantified 6 dendrites originating from pyramidal neurons in layer 5 accompanied by 18 dendrites originating in layer 2 and 3, per dendritic bundle (Peters and Sethares 1996, fig 11,19). Dendritic bundles often consist of apical dendrites originating from pyramidal neurons that project to the same target area (Innocenti and Vercelli 2010, fig 2).
} 


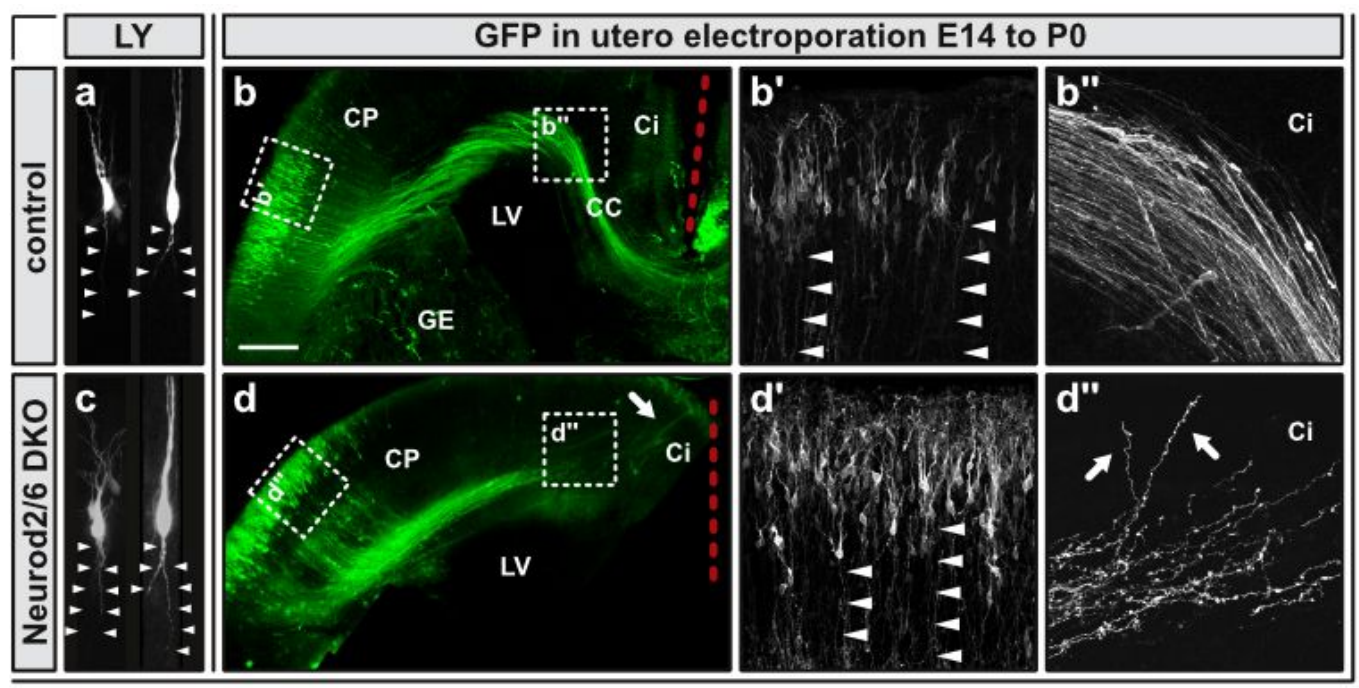

Figure 25: Callosal axon growth in Neurod2/6 double-deficient mice

(a, c) Single pyramidal neurons located in the upper cortical layers of newborn control (a) and Neurod2/6 double-deficient (c) mice were iontophoretically filled with the fluorescent cationic dye Lucifer Yellow (LY). (b, d) Upper layer pyramidal neurons were labeled by in utero electroporation of mGFP into the neocortex of control (b) and Neurod2/6 double-deficient (d) embryos at E14. Initial axon growth in the CP (arrow heads in a, b', c, d') and within the proximal callosal tract was not affected in Neurod2/6 double-deficient brains at P0 (c, $\left.d^{\prime}\right)$. When approaching the ipsilateral cingulate cortex, however, callosal axons defasciculated, failed to grow towards the midline and often followed random trajectories into the ipsilateral CP (arrows in d, d"). Pictures a, b', b", c, d', d" shown maximum intensity projections of image stacks recorded using a confocal microscope; b, d show tiled digital photographs acquired using an epifluorescent microscope. Iontophoresis (a, c) was performed together with Ming Zhang, Department of Neurophysiology, University of Göttingen (chapter 4.8.5).

is an inversion of the first one. Axons turn away from the midline, grow laterally in the IZ, defasciculate to target different cortex areas, and eventually enter the CP. ${ }^{1}$

In Neurod2/6 double-deficient mice, pyramidal cells expressing typical markers of callosal projection neurons were situated in the upper layers of the cortical plate (sect 2.4.2.4). However, they did never form a CC. The primary question was, whether putative callosal projection neurons were able to grow axons at all, and if yes, at what stage callosal axon growth was disturbed.

\subsubsection{Axon Growth}

To visualize single axons inside the cortical plate, pyramidal neurons in upper layers of thick vibratome sections were iontophoretically filled with the ionic fluorescent dye Lucifer yellow using a patch pipette. Image stacks were scanned using a confocal microscope and 3D reconstruction was performed. Axons could be identified for most fluorescently filled cells. Inside the $\mathrm{CP}$, the initial axonal trajectories were oriented centripetally and comparable in controls and Neurod2/6 double deficient mice (fig 25a, c). The further course of these axons could not be studied using this technique because more distal aspects were only weakly labeled and many axons did not grow within the same section plane.

\footnotetext{
${ }^{1}$ Richards et al. 2004, fig 2 "Developmental stages in the formation of the corpus callosum"
} 
Larger populations of pyramidal neurons were labeled by in utero electroporation. An expression construct coding for membrane targeted GFP (mGFP) was injected into one lateral ventricle at E14, the time when upper layer neuron precursor cells are normally generated in the VZ/SVZ. The DNA was electroporated into cortical cells by applying a weak direct current. The embryos were allowed to develop in utero for several days, and tissue was processed directly after birth.

Upper layer pyramidal neurons were clearly visible by direct GFP fluorescence. In electroporated control brains, callosal axons could be followed from the ipsilateral $\mathrm{CP}$, via IZ, cingulate cortex (Ci) and midline to the contralateral hemisphere (fig 25b). In Neurod2/6 double-deficient pups, axons left the CP (fig 25d'), turned medially in the IZ and fasciculated to form a compact fiber bundle (fig 25d). This putative callosal tract grew in medial direction, but failed to turn towards the midline and instead stalled in the SVZ of the medial neocortex. Axons defasciculated and the majority did not grow any further. A relatively small population grew astray into the ipsilateral cortex (fig $25 \mathrm{~d}$ "). There were no signs of midline interaction or PB formation (see also sect 2.4.4.4).

\subsubsection{Fasciculation}

One of the first published target genes of Neurod6 is growth associated protein 43 (Gap43), ${ }^{1}$ an axonal protein enriched in active growth cones. ${ }^{2}$ Inactivation of Gap43 in mice is lethal and results in non-selective fasciculation of callosal axons, formation of PBs, and agenesis of all cortical commissures. ${ }^{3}$ Heterozygous Gap43 deficient animals show milder abnormalities in cortical connectivity, develop autism-like symptoms, and show severe learning deficits. ${ }^{4}$ In humans, Gap43 has been associated with autism spectrum disorders ${ }^{5}$; some mutations result in agenesis of the $\mathrm{CC}$ and mental retardation. ${ }^{6}$ Overexpression of functional Gap43 in mice can enhance spatial and working memory ${ }^{7}$; overexpression of a non-phosphorylatable variant of Gap43 results in memory loss, defasciculation and aberrant axon growth. ${ }^{8}$

\footnotetext{
${ }^{1}$ The HLH proteins Id2, Tcf3 and Tcf12 bind an E-box in the Gap43 promoter and repress endogenous Gap43 expression in N18 cells (Chiaramello et al. 1996). Constitutive overexpression of Neurod6 in PC12 cells induces neurite outgrowth and Gap43 expression without NGF treatment (Uittenbogaard and Chiaramello 2002). Neurod6 binds the same E-box and induces Gap43 expression in N18 cells. (Uittenbogaard et al. 2003).

${ }^{2}$ Gap43 protein is localized in axons, enriched in axonal growth cones, and absent from dendrites (Goslin et al. 1988). Gap43 is phosphorylated in stationary growth cones and dephosphorylation leads to growth cone collapse (Dent and Meiri 1992).

${ }^{3}$ Shen et al. 2002: Gap43 deficient mice die perinatally. Newborn pubs lack AC, CC and HC. Callosal axon fail to cross the midline and instead form bilateral PBs. Midline fusion and axonal response to Slit2 secretion from the glial wedge are not affected.

${ }^{4}$ McIlvain et al. 2003; Shen et al. 2002; Zaccaria et al. 2010

${ }^{5}$ Allen-Brady et al. 2009; Schellenberg et al. 2006; Autism Genome Project Consortium et al. 2007; Trikalinos et al. 2006

${ }^{6}$ Genuardi et al. 1994; Molin et al. 2012

${ }^{7}$ Routtenberg et al. 2000: Transgenic mice expressed variants of the chicken Gap43 cDNA under control of a Thy 1 promoter fragment, which is selectively active in postnatal and adult neurons. Adult mice overexpressing wild type Gap43 made less mistakes in an 8-arm Olton radial maze $(-75 \%)$ and adapted faster to a paradigm shift $(-50 \%)$. Overexpression of a phosphorylation resistant or a permanently pseudo-phosphorylated form Gap43 had no significant effect.

${ }^{8}$ Holahan et al. 2010: Gap43 is phosphorylated by protein kinase C at Serin-41 in mice (Serin-42 in chicken). Neuron specific overexpressing of a phosphorylation resistant variant of chicken
} 
Unexpectedly, Gap43 mRNA levels were roughly comparable in Neurod2/6 double-deficient mice and control littermates at E16 and E19 (fig 26b, e). IHC confirmed axonal protein localization and revealed reduced Gap43 levels in the neocortex of newborn Neurod2/6 double-deficient mice (fig 26a). The used Gap43 antibody (GAP-7B10) recognizes both splice variants in the phosphorylated and dephosphorylated state, according to supplier information. The total number of Gap43-positive neurites was clearly reduced in the double-deficient neocortex. Remaining axons formed fasciculated fiber bundles in the $\mathrm{MZ}$ and SVZ (brackets in fig 26a). However, Gap43-positive axons were absent from the double-deficient IZ (asterisk in fig 26a). Focal Gap43 signals in the Neurod2/6 double-deficient $\mathrm{CP}$ (double arrow in fig 26a) demonstrated that abnormal ipsilateral projections were able to fasciculate and reach the MZ. In utero electroporation of mGFP into Neurod2/6 double-deficient embryos had shown that callosal fiber bundles defasciculated in the medial SVZ of the ipsilateral neocortex (sect 2.4.4.5; fig 25d). Electroporated axons grew stray into the ipsilateral cingulate cortex, apparently lacking sufficient guidance (arrows in fig 25d").

Initiation and maintenance of axonal fasciculation depend on axon-axon interactions, which are mediated by sets of axonal cell adhesion molecules (CAMs). ${ }^{1}$ L1cam is an immunglobulin domain containing CAM that is expressed by most pyramidal neurons and that is often used as molecular marker to visualize cortical fiber tracts. Contactin $2(\mathrm{Cntn} 2)$ is a related protein that is predominantly expressed by callosal projection neurons and often used to identify outgrowing axons in the CC.

IHC in brain sections of newborn Neurod2/6 double-deficient mice revealed that Cntn2-positive axons were totally absent from the neocortical SVZ/IZ (asterisks in fig 26d). In line with normal development of the HF (sect 2.4.2.1f), Cntn2 signals in the hippocampus were not reduced (arrow heads in fig 26d). Llcam was detectable in all parts of the Neurod2/6 double-deficient neocortex. QRT-PCR confirmed strongly $(\sim 80 \%)$ reduced Cntn2 mRNA levels in laser captured tissue from the CP (fig 26c). Contactin 1 (Cntn1) and L1cam mRNA levels were not significantly reduced in the same tissue samples.

The loss of neocortical Cntn2 expression might contribute to the premature defasciculation of initially fasciculated callosal fiber tracts in Neurod2/6 doubledeficient mice. However, Cntn2 is not necessary for callosal axon growth and for $\mathrm{CC}$ formation, as Cntn2 deficient mice have been reported to develop a grossly normal CC. ${ }^{2}$

\subsubsection{Midline Glia}

Fusion of the developing cerebral hemispheres and midline crossing of callosally projecting axons depend on several populations of specialized glia cells that persist

Gap43 (S42A) in mice lead to reduced performances in Morris water maze, ectopic axon growth in the hippocampus and defasciculation of hippocampal mossy fibers.

${ }^{1}$ Pollerberg et al. 2013 This review discusses the roles of immunglobulin domain containing CAMs in axonal guidance and fasciculation. An emphasis is put on mRNA transport and local translation in the axon and growth cone to allow for dynamic changes of protein levels in response to cell-extrinsic signals.

${ }^{2}$ Fukamauchi et al. 2001, fig 2B: The CC is visible in coronal brain section at P2. The midsagittal dorsoventral diameter, however, is reduced in all shown sections of Cntn2 deficient animals. 


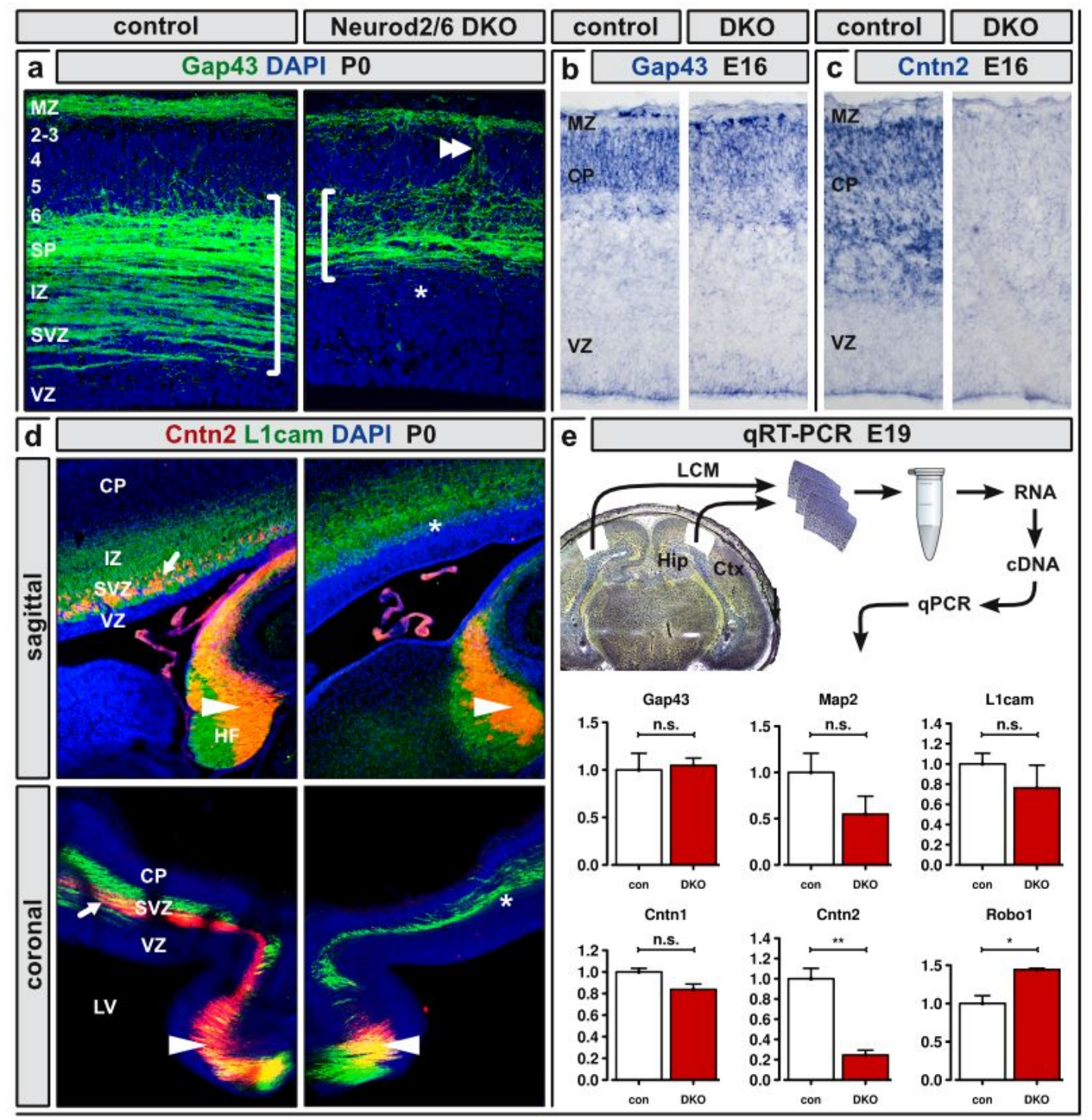

Figure 26: Loss of Contactin 2 in Neurod2/6 double-deficient mice

Cryostat sections of newborn control and Neurod2/6 double-deficient mice. (a) IHC for Gap43 protein (green) with DAPI (blue). (b) ISH for Gap43 mRNA at E16. (c) ISH for Cntn2 mRNA at E16. (d) Double IHC for Cntn2 (red) and L1cam (green) with nuclear DAPI stain (blue) in sagittal and coronal brain sections. Cntn2 protein normally marks fasciculated fiber tracts located in the neocortical IZ and in the fimbria of the hippocampus. In Neurod2/6 double-deficient animals, hippocampal Cntn2 expression was not changed, but Cntn2 was totally absent from the neocortex. (e) Relative quantification of mRNA expression levels by qRT-PCR using laser dissected tissue from the CP of E19 mice. Asterisks mark reduced staining intensity in the IZ. Arrows points to aberrant axon growth in the CP. The double arrow denotes an abnormal, radially oriented axon bundle in the CP.

during cortex development and surround the early corpus callosum. These midline glia cells produce various signaling molecules that influence callosal axonal growth. Repulsive cues from midline glia prevent axons from growing into other surrounding structures and help to channel axon growth to form the compact corpus callosum ${ }^{1}$

\footnotetext{
${ }^{1}$ Lindwall et al. 2007, sect "Midline glial development and commissure formation"
} 


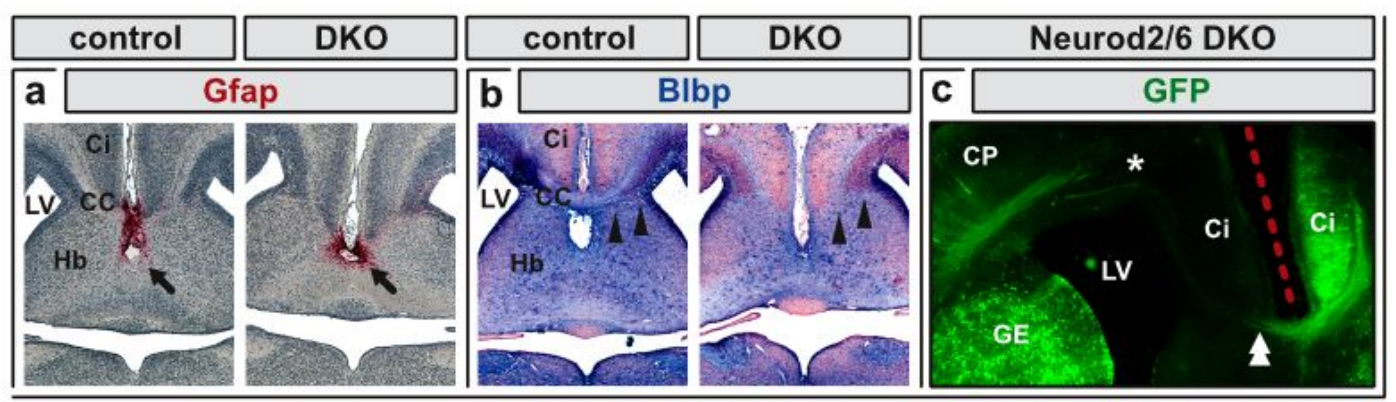

Figure 27: Midline fusion in Neurod2/6 double-deficient mice

(a, b) Horizontal paraffin sections of brains from newborn control and Neurod2/6 double-deficient mice. IHC for (a) Gfap (red) and (b) Blbp (blue) demonstrate the presence of midline glia in Neurod2/6 double-deficient brains. (c) In utero electroporation of GFP into the Ci labels axons of callosal pioneer neurons that crossed to the contralateral hemisphere. Arrows point to midline zipper glia cells. Arrow heads denote the glial wedge. The double arrow marks axons of callosal pioneer neurons that were able to cross the midline in Neurod2/6 double-deficient mice.

Midline glia structures could be identified in the Neurod2/6 double-deficient cortex by IHC for glial fibrillary acidic protein (Gfap) and brain lipid binding protein (Blbp). Midline zipper glia was correctly situated between third ventricle and septum, and the two cortical hemisphere had fused (fig 27a). The glial wedge was also present and positioned directly medially to the lateral ventricle (fig $27 \mathrm{~b}$ ).

Most transgenic mice with developmental CC defects form PBs in the medial cortex. PBs are aggregates of aberrantly growing axons that reach the midline but fail to cross to the contralateral hemisphere. ${ }^{1}$ In Neurod2/6 double-deficient mice, PBs or other aberrant axon tracts were never observed (fig 18c, f; fig 19; fig 28a). In utero electroporation of mGFP into the CP demonstrated that neocortical axons did not reach any midline associated structures (asterisks in fig 25d; fig 27c). Medially oriented electroporation into the Ci showed that midline glia was functional in the absence of Neurod2/6: Pioneer axons originating from the cingulum were able to cross the midline to the contralateral hemisphere. They successfully formed an early callosal anlage, which was never populated with callosal axons from the neocortex (double arrow head in fig 27c).

\subsubsection{Midline Crossing}

Unexpectedly, qRT-PCR screening revealed increased expression levels of roundabout 1 (Robo1) mRNA in the CP of Neurod2/6 double-deficient mice (fig 26e; fig 28). Robol is an immunoglobulin domain containing CAM and a receptor for Slit proteins. Slits are repellent signaling molecules that are secreted from midline structures and cortical neurons. ISH confirmed strongly increased Robol expression in the CP of Neurod2/6 double-deficient mice at E16 (fig 28a) and P0 (fig 28b). ${ }^{2}$

\footnotetext{
${ }^{1}$ Probst 1901; Lindwall et al. 2007

${ }^{2}$ Bormuth et al. 2013, fig 7D: Here we also show an overview picture illustrating Robolupregulation along the mediolateral axis of the neocortex, but relatively normal expression in the hippocampus.
} 


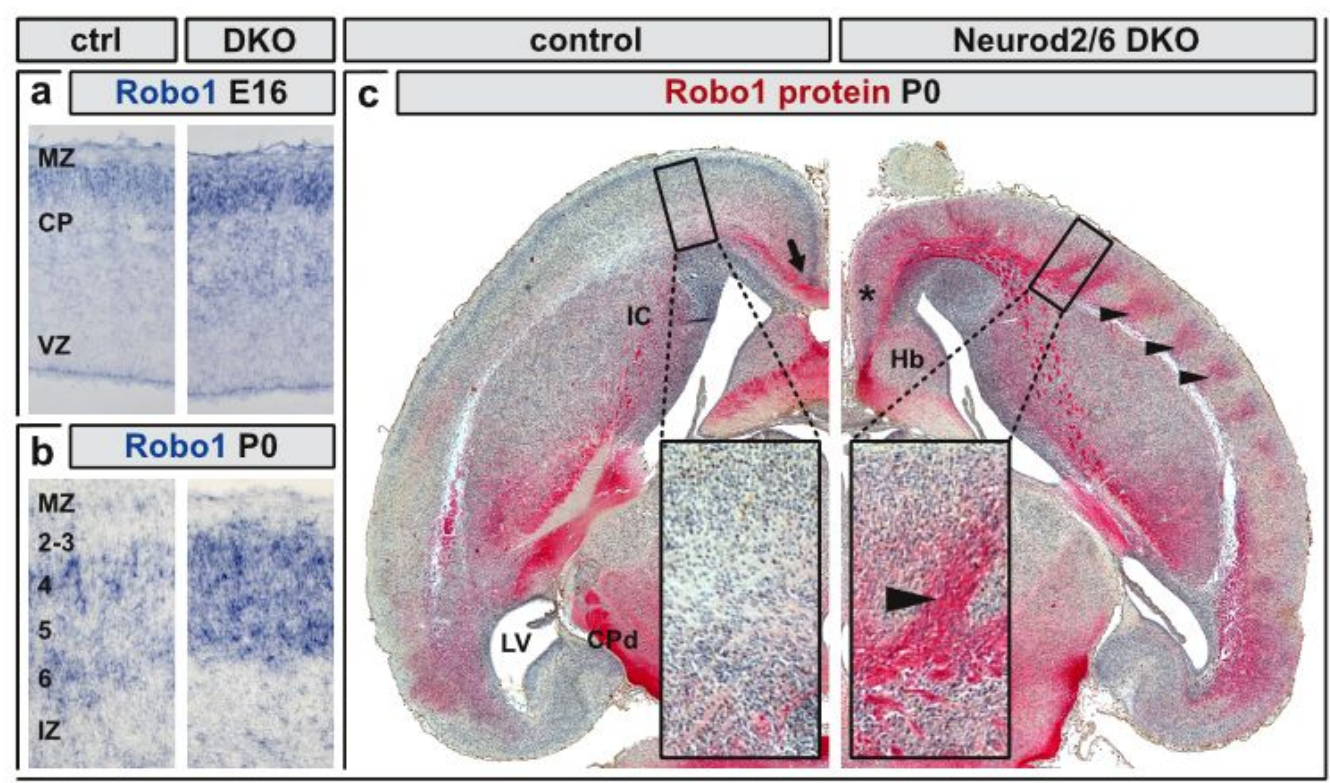

Figure 28: Ectopic Robo1 expression in Neurod2/6 double-deficient mice

$(\mathbf{a}, \mathbf{b})$ ISH shows transcriptional upregulation of Robo 1 mRNA in the Neurod2/6 double-deficient CP at E16 and P0. (c) IHC for Robo1 (red) in horizontal paraffin sections identifies many Robo1-positive axons aggregates in the neocortex of newborn Neurod2/6 double-deficient mice (arrow heads). Robo1 protein is normally targeted to the distal axon and thus barely detectable in the CP, but enriched in the CC (arrow), IC and CPd of control brains.

In spinal cord it was shown that the subcellular localization of Robol receptors is confined to the contralateral aspect of commissural axons that have already crossed the midline. ${ }^{1}$ Chromogenic IHC for Robol in horizontal brain sections of newborn control mice confirmed the presence of Robol protein in distal axons of the CC and IC (left panel of fig 28c ). Robol staining was restricted to the paramedian aspect of the CC (arrow in fig 28c), and is was virtually absent from the coronal radiation (CR) and from proximal axons, dendrites and neuronal cell bodies located in the CP. ${ }^{2}$ This pattern suggested that also in the CC Robol receptors are trafficked to the contralateral (post-crossing) axonal compartment. However, the co-localization of ipsilateral (pre-crossing) and contralateral (postcrossing) axons in all regions of the CC makes it difficult to provide direct proof.

In newborn Neurod2/6 double-deficient mice, Robol IHC showed an entirely different pattern. The protein was abundant in axons located in the CC, CR, IC and CP (right panel of fig 28c). Due to complete agenesis of CC and AC (sect 2.4.2.1), axonal Robo1 in the neocortex of Neurod2/6 double-deficient mice can be considered of strictly ipsilateral origin. Interestingly, Robo1-positive axons were not uniformly distributed within the $\mathrm{CP}$, but they formed prominent, radially orientated, brush like aggregates (arrow heads in fig 28).

\footnotetext{
${ }^{1}$ Long et al. 2004, fig 4: In the spinal cord, Robol and Robo2 proteins are preferentially localized to the contralateral (ascending) aspects of commissural axons while Cntn2 (Tag1) is preferentially localized to the ipsilateral segment.

${ }^{2}$ Andrews et al. 2006, fig 2: Robol IHC in E17 coronal and horizontal brain sections including the $\mathrm{CC}$ confirms protein localization in distal axons.
} 


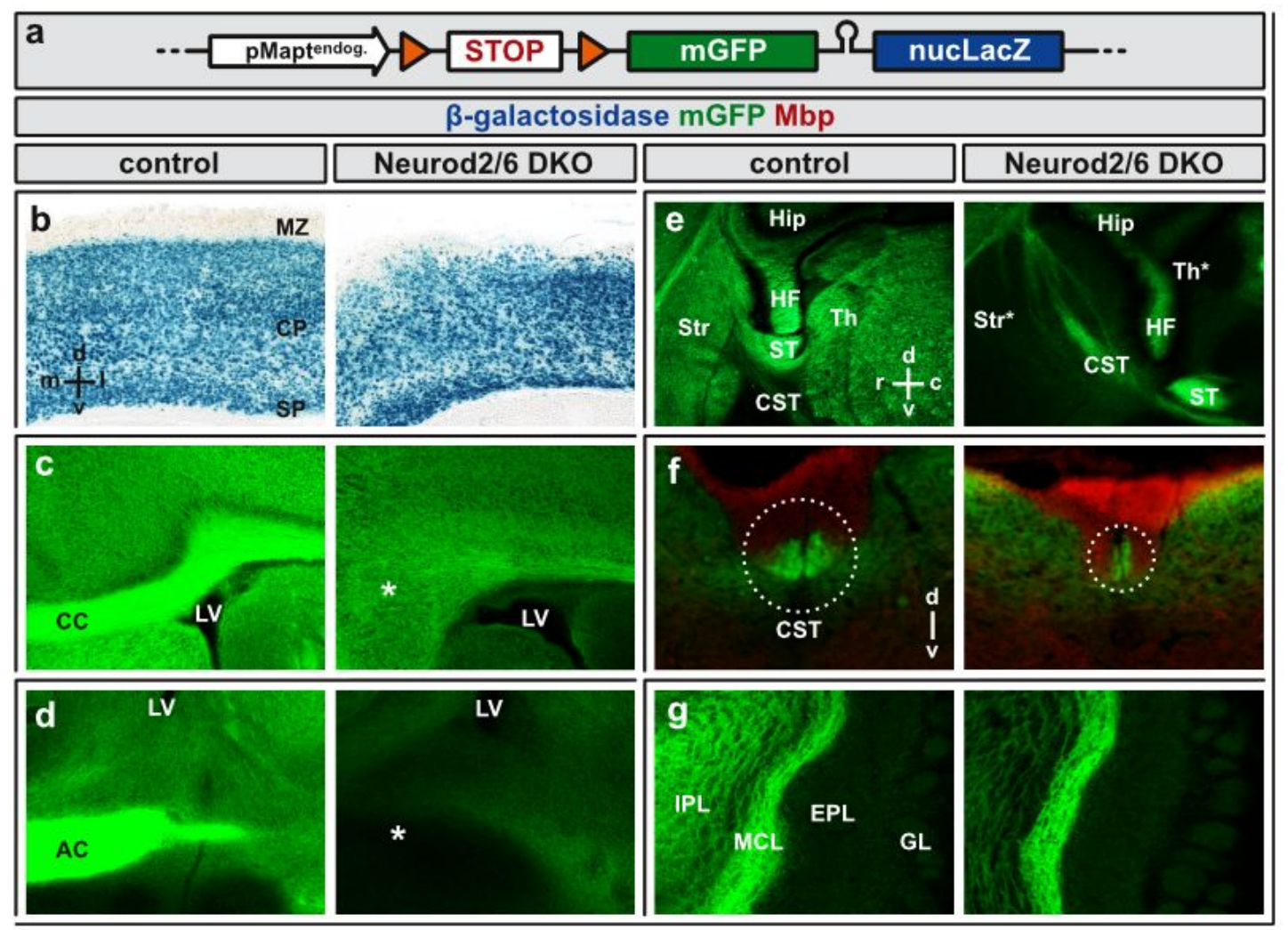

Figure 29: Cortical axonal projections in Neurod2/6 double-deficient mice

(a) Schematic view of the reporter construct used to visualize cell bodies and axons of Neurod6-lineage pyramidal neurons. (b-g) Cryostat sections of two week old control and Neurod2/6 double-deficient mice: (b) X-gal histochemistry resulted in a nuclear staining of recombined pyramidal neurons situated in the cerebral cortex. (c-g) MGFP fluorescence (green) labeled the axons of only those recombined cells. (c, d) Coronal sections at the levels of the CC and AC confirmed the total absence of both neocortical commissural fiber tracts. (e) Sagittal sections at the level of the internal capsule demonstrated strongly reduced cortical connectivity to the thalamus and striatum in Neurod2/6 double-deficient mice. Fluorescent signals in the hippocampal fimbria and the corticospinal tract were not reduced. (f) Cross sections of the thoracic spinal cord demonstrated that at least a subset of cortical motoneuron axons crossed at the pyramidal decussiation and reached the dorsal corticospinal tract (CST) in the absence of Neurod2 and Neurod6. IHC for Mbp (red) labeled myelinated fiber tracts and identified the adjacent posterior funiculus in the same sections. (g) The connectivity of the olfactory bulb was not severely disturbed. Asterisks denote regions where the density of cortical axons is high in controls, but low in Neurod2/6 double mutant mice.

\subsubsection{Subcortical Projections}

The cerebral cortex harbors an interweaved network of efferent, afferent, and intracortical projections. Due to the tissue's complexity, it is very difficult to identify distinct axon populations by means of classical histology. Cell type specifically expressed marker proteins can visualize certain axon populations, but the expression level of marker genes are often changed as part of the phenotype to be analyzed. Expression levels of the callosal marker genes Cntn2 and Robo1, for example, are substantially changed in neocortical pyramidal neurons of Neurod2/6 double-deficient animals ${ }^{1}$ (sect 2.4.4.3).

\footnotetext{
${ }^{1}$ Bormuth et al. 2013, fig 7
} 
Cre reporter mice ${ }^{1}$ were used to selectively visualize axons originating from Neurod6-Cre positive neurons in Neurod2/6 double-deficient mice. ${ }^{2}$ The reporter allele expresses mGFP conditionally under control of the endogenous microtubule associated protein $\mathrm{t}$ (Mapt)-promoter, which is strongly active in cortical neurons. Nuclear-targeted $\beta$-galactosidase is expressed from the same locus via an internal ribosomal entry site (IRES) sequence (fig 29). After genomic recombination, the expression levels of mGFP and $\beta$-galactosidase do not depend on the Neurod6promoter, but follow the expression levels of Mapt.

$\mathrm{X}$-gal histochemistry was used to visualize $\beta$-galactosidase. Most neurons in the double mutant cortical plate were $\mathrm{X}$-gal positive and had thus undergone Cre mediated recombined. The staining intensity was comparable in controls and double mutants (fig 29b), which suggests that the activity of the Mapt-promoter is largely independent of Neurod2/6.

In coronal brain sections of Neurod2/6 double-deficient mice, GFP fluoresce confirmed the absence of CC (asterisk in fig 29c) and AC (asterisk in fig 29d). Axonal GFP signals in the striatum and thalamus were strongly reduced when compared with controls (asterisk in fig 29e). Together with reduced Vglut1 protein levels in the striatum of older Neurod2/6 double-deficient mice (sect 2.4.3), this suggests a loss in subcortical axonal connectivity originating from pyramidal neurons located in layer 6 of the neocortex. ${ }^{3}$ Axons of subcerebrally projecting neocortical pyramidal neurons reached the spinal cord in Neurod2/6 doubledeficient mice, but the cross section area of the dorsal CST was dramatically reduced (fig 29f). The connectivity of the olfactory bulb (OB) was not substantially changed (fig 29g).

\subsubsection{Synaptic Function}

Due to the early lethality of most Neurod2/6 double-deficient mice, neuronal activity was recorded in primary neocortical neuron cultures prepared from newborn Neurod2/6 double-deficient mice and littermate controls. Dissociated cells were manually counted and seeded at equal numbers per well. The cultures were kept in vitro for 3-4 weeks to allow for the formation of interconnected neuronal networks.

${ }^{1}$ Hippenmeyer et al. 2005: Following Cre-mediated genetic recombination, membrane targeted GFP and nuclear lac $Z$ are bicistronically expressed from the endogenous Mapt-promoter (pMapt-LoxP-STOP-LoxP-mGFP-IRES-nLacZ-polyA).

${ }^{2}$ The genetic loci of Mapt and Neurod2 are both located on chromosome 11 . The genetic distance is approximately $6 \mathrm{Mbp}$, which corresponds to an estimated genetic linkage of $6 \mathrm{cM}$ and a theoretical recombination probability of roughly $6 \%$. The two loci eventually integrated on the same allele. In order to minimize phenotypical interference, experimental animals were kept heterozygous for the transgenic (Mapt deficient) allele.

${ }^{3}$ The reporter allele was not explicitly demonstrated to be transcriptionally active in all pyramidal neuron subtypes of Neurod2/6 double-deficient mice. Absence of axonal GFP fluorescence in striatum and thalamus (fig 29e) can be explained either by the absence of corticostriatal axon terminals in these areas, or by the loss of Mapt-promoter activity in subcortically projecting pyramidal neurons. The reporter failed to be expressed in spinal interneurons of Neurod $1 / 2 / 6$ triple-deficient mice (fig 42e). It is thus important to not simple assume the endogenous Maptpromoter to be pan-neuronally active. Normal localization of Ctip2-, Fox2- and Sox5-positive (subcerebrally projecting) pyramidal neurons in the deepest layer of the Neurod2/6 doubledeficient neocortex in combination with strong and uniform $\beta$-galactosidase activity in the lower double-deficient CP, however, suggest defective axon growth as the most likely explanation. 


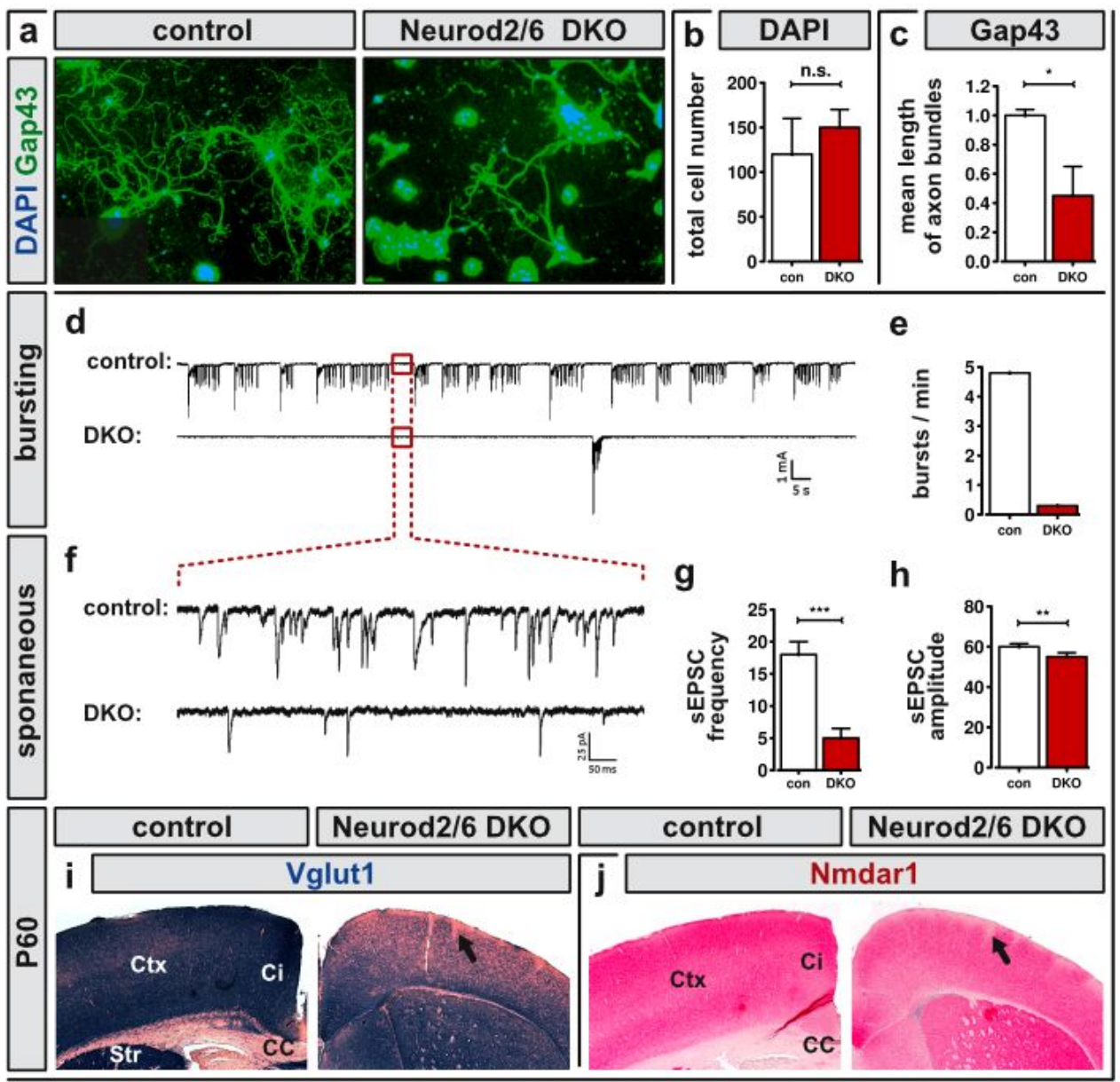

Figure 30: Pyramidal neurons are functional in absence of Neurod2/6

Primary cortical pyramidal neurons were cultured for 3-4 weeks. (a-c) Cell nuclei were stained using DAPI (blue in a) and neurites were labeled by IHC for Gap43 (green in a). The quantification of double stained coverslips demonstrated comparable total cell numbers (b), but significantly reduced neuronal connectivity (c). (d,e) Neuronal activity was recorded by patch-clamping individual cells. Control cultures showed strong episodic bursting activity, which was rarely observed in Neurod2/6 double mutants. (f) Spontaneous synaptic activity was recorded in interburst intervals. (g) The mean sEPSC frequency was strongly reduced while (e) the sEPSC amplitude was only mildly reduced in Neurod2/6 double-deficient cultures. (i,j) IHC for the presynaptic glutamate transporter Vglut1 (i) and the postsynaptic glutamate receptor Nmdar1 (j) in brain sections of adult control and Neurod2/6 double-deficient mice. Electrophysiological recordings were performed together with Ming Zhang, Department of Neurophysiology, University of Göttingen (chapter 4.8.5).

After fixation, DAPI staining confirmed that the total cell number was comparable in Neurod2/6 double-deficient and control cultures (fig 30a, b). However, IHC for Gap43 and quantification of Gap43-positive axon bundles showed a reduction of the overall connectivity by approximately $50 \%$ in Neurod $2 / 6$ double-deficient cultures (fig 30a, c).

Patch-clamp recordings performed in collaboration with Ming Zhang (fig 30; chapter 4.8.5) demonstrated spontaneous episodic bursting activity in control cultures, but rarely in Neurod2/6 double-deficient cultures (fig 30d, e). Spontaneous excitatory postsynaptic currents (sEPSCs) were selectively measured in interburst intervals (fig 30f). The frequency of sEPSCs was reduced by more than two-thirds (fig 30h), which is consistent with reduced structural connectivity and 
lower synaptic density. The mean sEPSC amplitude was only mildly reduced (fig 30g) suggesting that glutamatergic synapses are functional and that the most essential pre- and postsynaptic components are provided and maintained in the absence of Neurod2 and Neurod6.

The latter was exemplarily confirmed by immunostaining for vesicular glutamate transporter 1 (Vglut1) and ionotropic NMDA glutamate receptor, subunit 1 (Nmdar1) in brain sections of adult mice (fig 30i, j). Vesicular glutamate transporters are associated with presynaptic vesicles of all glutamatergic neurons and are necessary for glutamatergic neurotransmission. ${ }^{1}$ Vglut 1 was expressed in adult Neurod2/6 double-deficient mice, but protein levels were reduced in neocortical target areas such as the striatum, the thalamus or the $\mathrm{CP}$, and barely detectable in the MZ (arrow in fig 30i). N-Methyl-D-aspartic acid (NMDA) binding glutamate receptors are critical for synaptic plasticity and memory formation. ${ }^{2}$ Nmdar 1 was expressed in adult Neurod2/6 double-deficient mice, also protein levels were mildly reduced in neocortical target areas, especially the MZ (arrow in fig 30j).

\subsubsection{Arealization of the Neocortex}

During the first postnatal week, afferent thalamocortical axons form topographic connections to layer 4 pyramidal neurons of the mouse somatosensory cortex. This focal increase of intracortical connectivity leads to morphological changes and results in the formation of the typical somatosensory whisker-barrel cortex (sect 3.4). Postnatal maturation of the somatosensory cortex and the segregation of whisker-barrels had previously been reported to be disturbed in Neurod2 single-deficient mice (sect 1.5.2).

Cytochrome oxidase $(\mathrm{CO})$ is a mitochondrial enzyme that is involved in neuronal energy metabolism and that can be used to indirectly monitor chronic neuronal activity. ${ }^{3}$ CO histochemistry in brains sections of P8 mice (fig 3a) was used to visualize the functional maturation of the somatosensory cortex during the first postnatal week (fig $31 \mathrm{~b}$ ). Synaptic activity of thalamocortical axons normally leads to strong CO staining in layer 4 of the somatosensory cortex, particularly in whisker-barrels. Coronal brain sections of control mice harboring only one copy of Neurod 2 and no Neurod6 (genotype Neurod2 ${ }^{\text {Wt/Null }} \times$ Neurod6 $6^{\text {Cre/Cre }}$ ) demonstrated the typical $\mathrm{CO}$ enrichment in layer 4 of the somatosensory cortex area. Neurod2/6 double-deficient mice (genotype Neurod2 ${ }^{\text {Null/Null }} \times$ Neurod6 ${ }^{\text {Cre/Cre }}$ ), however, showed no signs of somatosensory maturation. The CO staining was uniformly distributed throughout the neocortex and the signal intensity in layer 4 of Neurod2/6 double-deficient brain sections was comparable to that in nonenriched regions of controls. The $\mathrm{CO}$ enrichment in layer 1 of the cingulate cortex was comparable in controls and Neurod2/6 double-deficient mice (fig $31 \mathrm{~b}$ ).

\footnotetext{
${ }^{1}$ Wojcik et al. 2004; Daniels et al. 2006

${ }^{2}$ Warburton et al. 2013

${ }^{3}$ Wong-Riley and Welt 1980: CO activity was enriched within the centers of cortical whiskerbarrels. The removal of whiskers resulted in a decrease of $\mathrm{CO}$ activity in the corresponding barrel. Similar results were obtained in the auditory system, where electrical stimulation of quiescent auditory nerves resulted in a re-activation of cortical neuronal activity and CO staining intensity.
} 


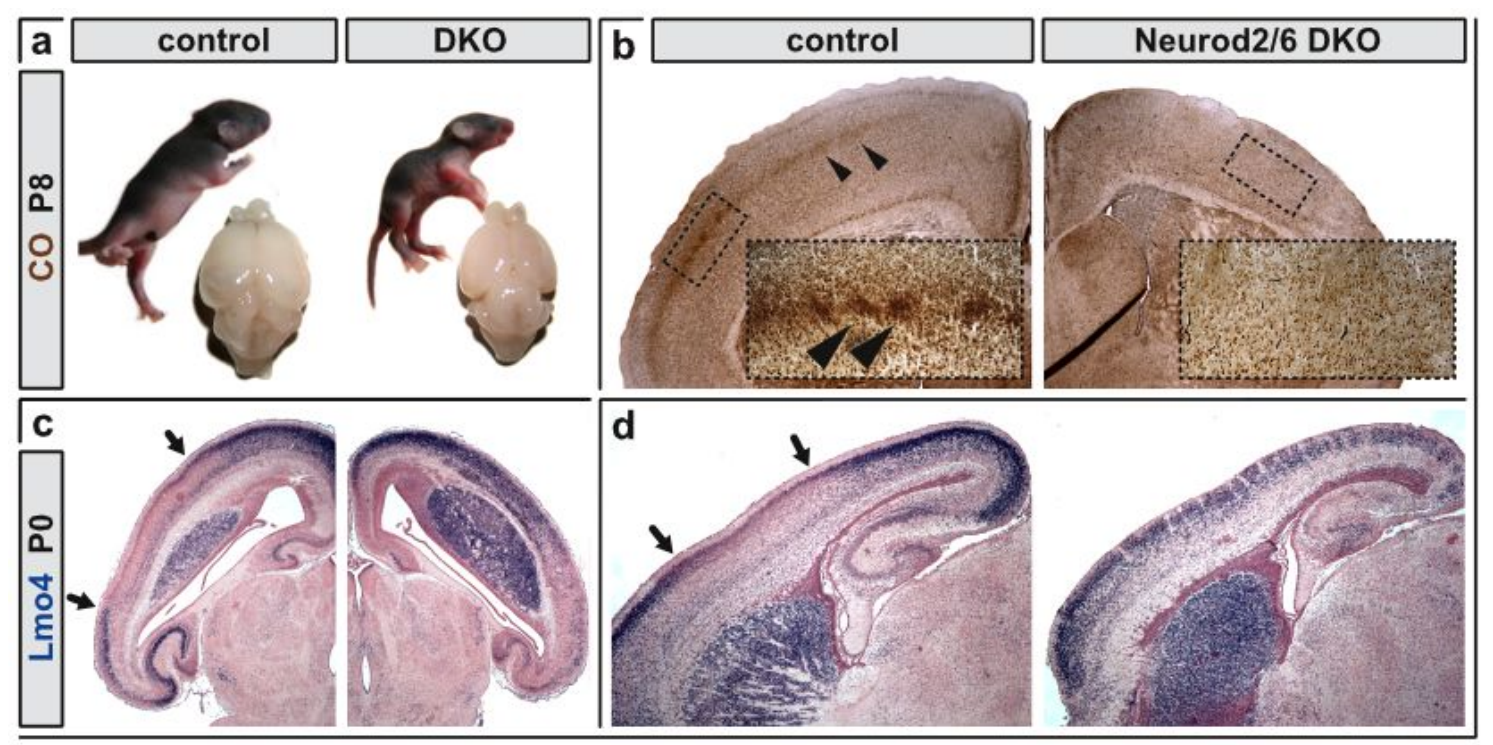

Figure 31: Neocortical arealization in Neurod2/6 double-deficient mice

(a, b) Immunohistochemistry for Lmo4 (blue) in horizontal (a) and sagittal (b) brain sections of newborn control and Neurod2/6 double-deficient (DKO) mice. (c) Control (Neurod2 $2^{\mathrm{Wt} / \mathrm{Null}} \times$ Neurod $6^{\mathrm{Cre} / \mathrm{Cre}}$ ) and Neurod2/6 double-deficient (Neurod2 ${ }^{\text {Null/Null }} \times$ Neurod6 $\left.6^{\mathrm{Cre} / \mathrm{Cre}}\right)$ mice and brains at $\mathrm{P} 8$. (d) CO histochemistry in coronal sections of the brains shown in (c) visualizes the postnatal formation of somatosensory whiskerbarrels in controls, but not in Neurod2/6 double-deficient mice. Arrows denote the approximate borders of the somatosensory cortex area. Arrow heads point to cellular aggregates in layer 4 of the somatosensory whisker cortex, the so called whisker-barrels.

Lmo4 is a transcription factor that is broadly expressed in most parts of the developing neocortex, but almost absent from deeper layers of the somatosensory area of newborn mice (sect 2.4.2.4). ${ }^{1}$ Lmo4 is discussed to define cortical arealization, to regulate sensorimotor control, and to be necessary for proper barrel field formation. ${ }^{2}$ In coronal sections of newborn Neurod2/6 doubledeficient mice, Lmo4 was continuously expressed along the mediolateral axis of the neocortex (fig 31d). A small and poorly defines Lmo4-negative area could only be identified in horizontal brain sections of the ventral cortex (fig 31c).

\subsubsection{Upregulation of Neurod1}

During mouse embryogenesis, Neurod2 and Neurod6 are broadly expressed in the SVZ and in all layers of the developing CP. By contrast, Neurod 1 expression is normally restricted to the upper $\mathrm{VZ}$ and to the lower SVZ, but absent from the CP before birth (sect 1.4.1; sect 1.4.2; sect 2.4.7a). ${ }^{3}$

\footnotetext{
${ }^{1}$ Sun et al. 2005, fig 3: ISH for Lmo4 mRNA in whole mount (D) and sagittally sectioned (I) brains of $\mathrm{P} 1$ mice

${ }^{2}$ Huang et al. 2009, fig 3, 4: The somatosensory barrel field is smaller and whisker-barrels are less defined in $\mathrm{Lmo} 4$ deficient ice.

${ }^{3}$ Gummert 2003, fig 3:: Chromogenic ISH for Neurod1, Neurod2, Neurod6, and other neuronal $\mathrm{bHLH}$ proteins in sagittal brain sections from $\mathrm{E} 18$ wild type mice
} 


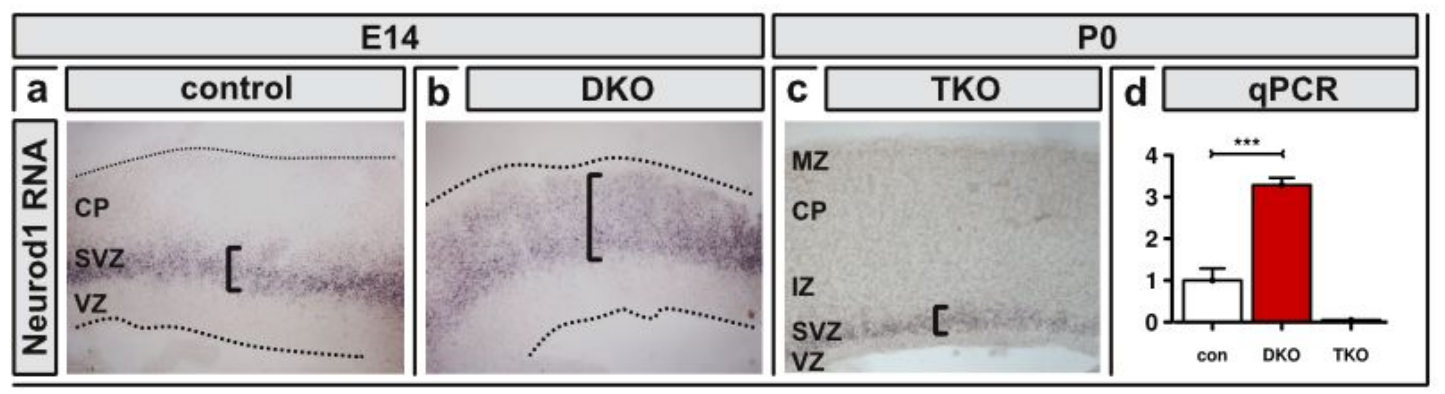

Figure 32: Neurod1 upregulation in Neurod2/6 double-deficient mice

$(\mathbf{a}, \mathbf{b})$ ISH for Neurod1 mRNA in frozen brain sections of E14 control and Neurod2/6 double-deficient mice: Neurod1 expression was ectopically increased in pyramidal neurons of the Neurod2/6 double-deficient CP. (c) Neurod1 ISH in brain sections from newborn Neurod1/2/6 triple-deficient animals: The conditional inactivation of Neurod1 in the Neurod6-lineage of cells entirely prevented Neurod1 expression in the CP but allowed for transient Neurod1 expression in pyramidal neuron precursors transitioning from the VZ to the SVZ. (d) Relative quantification of Neurod1 expression levels by qRT-PCR in laser capture dissected tissue from the $\mathrm{CP}$ of newborn control (con), Neurod2/6 double-deficient (DKO), and Neurod1/2/6 triple-deficient (TKO) mice. In-situ hybridization was partially performed together with Maike Gummert, Department of Neurogenetics, Max-Planck-Institute of Experimental Medicine (chapter 4.8.5).

In Neurod2/6 double-deficient mice, Neurod 1 mRNA was detectable in pyramidal neurons throughout the CP at E14 (fig 32b). This ectopic expression of Neurod 1 in differentiated pyramidal neurons might partially compensate for the loss of Neurod2/6 (sect 1.6). Abnormal transcriptional activity of Neurod 1 might thus have masked certain aspects of the Neurod2/6 double-deficient phenotype in the experiments that have been described so far.

\subsection{Simultaneous Inactivation of Neurod1/2/6}

$\mathrm{C}$ ONDITIONAL NEUROD 1/2/6 TRIPLE-DEFICIENT MICE were used to identify further redundant functionality. The main question was whether Neurod 1 alone could compensate for the loss of Neurod2/6 (sect 2.4.7). The described Neurod2/6 double-deficient animals (sect 2.4) were crossbred with Neurod1-Flox mice (sect 1.5.1). In Neurod1-Flox homozygous and at the same time Neurod6Cre positive offspring, the entire Neurod 1 coding sequence in specifically removed from the genomes of all Neurod6-lineage cells (fig 11; sect 2.1). Compensatory upregulation of Neurod 1 in the IZ and CP of Neurod2/6 double-deficient animals could thus be circumvented, while earlier Neurod 1 expression in the Neurod2/6-negative lower SVZ was maintained.

ISH and qRT-PCR verified dramatically decreased Neurod 1 expression in Neurod 1/2/6 triple-deficient mice when compared to Neurod2/6 double-deficient animals (fig 32c, d; fig 8c). Neurod 1 mRNA was absent from the triple-deficient CP but was still detectable in a thin band of cells located at the transition of $\mathrm{VZ}$ and SVZ (bracket in fig 32c). The selective inactivation of Neurod 1 in Neurod6-lineage cells was considered appropriate to restore a more natural Neurod 1 expression pattern in the absence of Neurod2/6. 
The analysis of Neurod 1/2/6 triple-deficient brains exposed several unknown functions of Neurod2/6. Those had indeed been taken over by ectopically expressed Neurod1, which can act as a functional replacement for Neurod2/6.

\subsubsection{Breeding}

Neurod 1/ 6 double-deficient mice with only one functional Neurod2 allele ${ }^{1}$ were fertile in both sexes, had a normal life span and were routinely used to breed triple-deficient offspring ${ }^{2}$ and Neurod $1 / 6$ double-deficient littermate controls. ${ }^{3}$ The latency of successful mating was considerably increased. In most cases, it took weeks before freshly paired females received vaginal plugs. Females were not particularly aggressive towards approaching males, but males appeared passive and not libidinous. Data obtained from 19 virgin males that were each successfully mated to two virgin females resulted in the first offspring after $37.1 \pm 4.6$ days (median 29). Assuming 19 days of pregnancy, fertilization must have happened after approximately 18 days (median 9). The birth frequency in permanent mating was $28.1 \pm 4.9$ days (median 21.8). Litters counted $7.4 \pm 0.23$ newborn pubs. Pregnant females were only moderately engaged in nest-building. Males were never observed to be aggressive against lactating females or against their offspring. The survival rate at weaning was $88.7 \% .48 .0 \%$ of all weaned offspring were females.

Neurod 1/2/6 triple-deficient mice were born at the expected Mendelian ratio of $25 \%$. Directly after birth, triple-homozygous pubs were hardly distinguishable from control littermates, but they experienced breathing abnormalities that, in principle, resembled those observed in Neurod2/6 double-deficient animals (sect 2.4). Apnea periods were longer and more frequent in triple-deficient pups, which quickly developed severe cyanosis and mostly died within minutes after birth.

Incidental observations of adult Neurod 1/6 double-deficient and at the same time Neurod2 heterozygous mice ${ }^{4}$ suggest altered social behavior (increased grooming, reduced nest building, potentially isoflurane induced seizures) and quantitative impairments in the formation of $\mathrm{AC}, \mathrm{CC}$ and cornu ammonis (CA). Cross section diameters of the anterior and posterior aspects of the AC were reduced by approximately $50 \%$ in adult Neurod $1 / 6$ double-deficient animals harboring one allele of Neurod2, when compared to littermates with both alleles. The longest midsagittal diameter of the CC was approximately $50 \%$ shorter and the splenium was absent. The number of hippocampal pyramidal neurons was remarkably reduced ${ }^{5}$ while the size of the HF appeared unchanged. Neuronal cell death in the Ci was significantly increased (sect 2.5.5). However, systematic

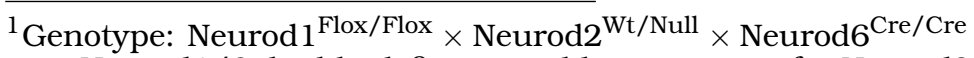
$=$ Neurod $1 / 6$ double-deficient and heterozygous for Neurod 2

${ }^{2}$ Genotype: Neurod $1^{\text {Flox/Flox }} \times$ Neurod2 $2^{\text {Null/Null }} \times$ Neurod6 $6^{\text {Cre/Cre }}$ $=$ Neurod $1 / 2 / 6$ triple-deficient $(\mathrm{TKO})$

${ }^{3}$ Genotype: Neurod $1^{\text {Flox/Flox }} \times$ Neurod $2^{\text {Wt } / W t} \times$ Neurod6 6 Cre/Cre $=$ Neurod $1 / 6$ double-deficient

${ }^{4}$ Genotype: Neurod1 $1^{\text {Flox/Flox }} \times$ Neurod $2^{\text {Wt/Null }} \times$ Neurod6 ${ }^{\text {Cre } / C r e ~}$ $=$ Neurod $1 / 6$ double-deficient and heterozygous for Neurod 2

${ }^{5}$ The hippocampal DG was absent (fig 34) due to the loss of Neurod1 (sect 1.5.1).
} 

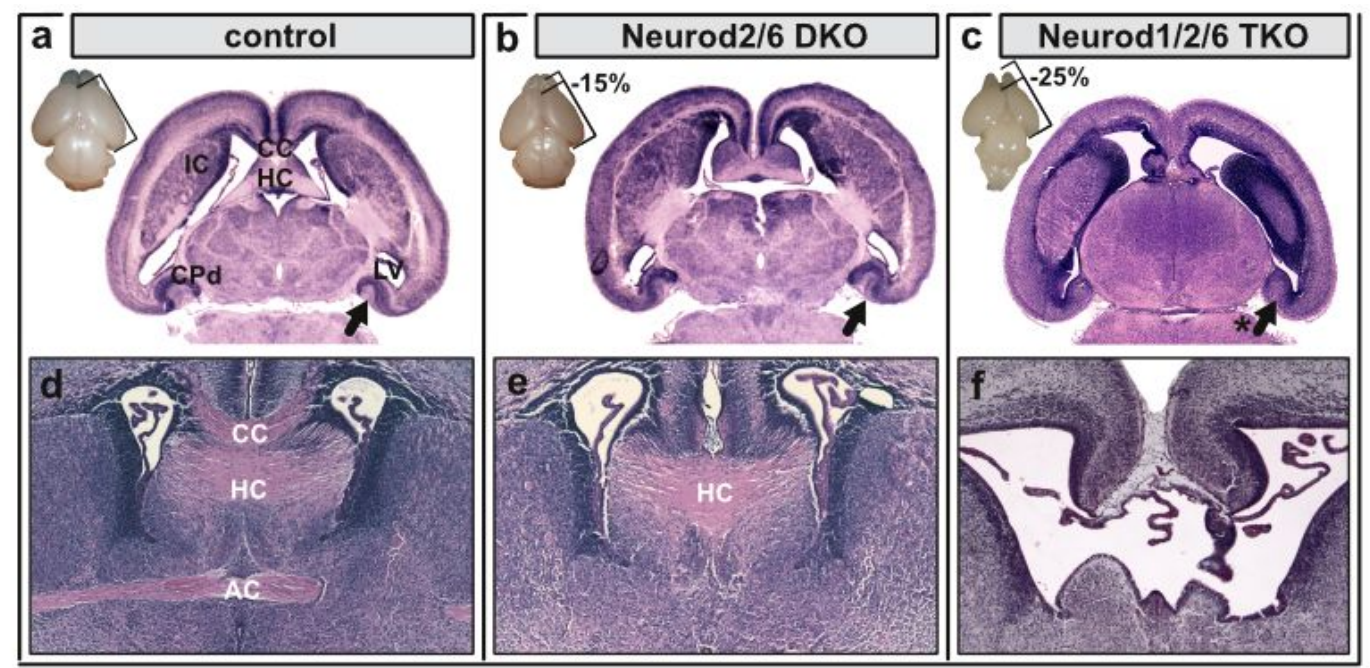

Figure 33: Brain anatomy of newborn Neurod1/2/6 triple-deficient mice

HE stained paraffin sections of newborn control $(a, d)$, Neurod2/6 double-deficient $(b, e)$ and Neurod1/2/6 triple-deficient $(\mathrm{c}, \mathrm{f})$ mice. The upper panel $(\mathrm{a}-\mathrm{c})$ shows horizontal brain sections from the brains depicted in the upper left corner. The lower panel (d-f) shows coronal close-ups of the commissural plate. Arrows point to the temporal pole of the hippocampus. Scales in b, c show the total rostrocaudal diameter of the cerebral cortex (long whiskers) in relation to a (short whisker). For better comparability, the sections in $\mathrm{a}, \mathrm{b}$ and $\mathrm{d}$, e are repeated from fig $18 \mathrm{~b}, \mathrm{c}$ and $\mathrm{e}, \mathrm{f}$, respectively.

investigations of these preliminary findings and their behavioral implications were beyond the scope of this study, and the following phenotypical analysis focuses on Neurod 1/2/6 triple deficient embryos.

\subsubsection{Brain Anatomy}

Macroscopically, brains of newborn Neurod 1/2/6 triple-deficient mice looked relatively normal (inset in fig 33c). The telencephalon had developed and did not show obvious malformations at its surface. The longest rostrocaudal diameter of the cerebral cortex was reduced by approximately $25 \%$ in comparison to control littermates and by approximately $12 \%$ in comparison to age matched Neurod $2 / 6$ double-deficient pups (fig 33a-c).

Brain volume had predominantly been lost to the expense of the later white matter. In addition to the previously described inability of Neurod2/6 doubledeficient mice to form neocortical projections to the contralateral hemisphere, to the thalamus and to the striatum (sect 2.3.2; sect 2.4.4.1), triple-deficient animals lacked most intracortical and all hippocampal fiber tracts (fig 33c, f). Gray matter was lost in the caudomedial cortex and hippocampal formation (arrows in fig 33c; fig 34d). The hippocampal pyramidal neuron layer, which is normally characterized by a particularly high density of Neurod $1 / 2 / 6$ expressing neurons, was hardly identifiable in triple-deficient brains. 


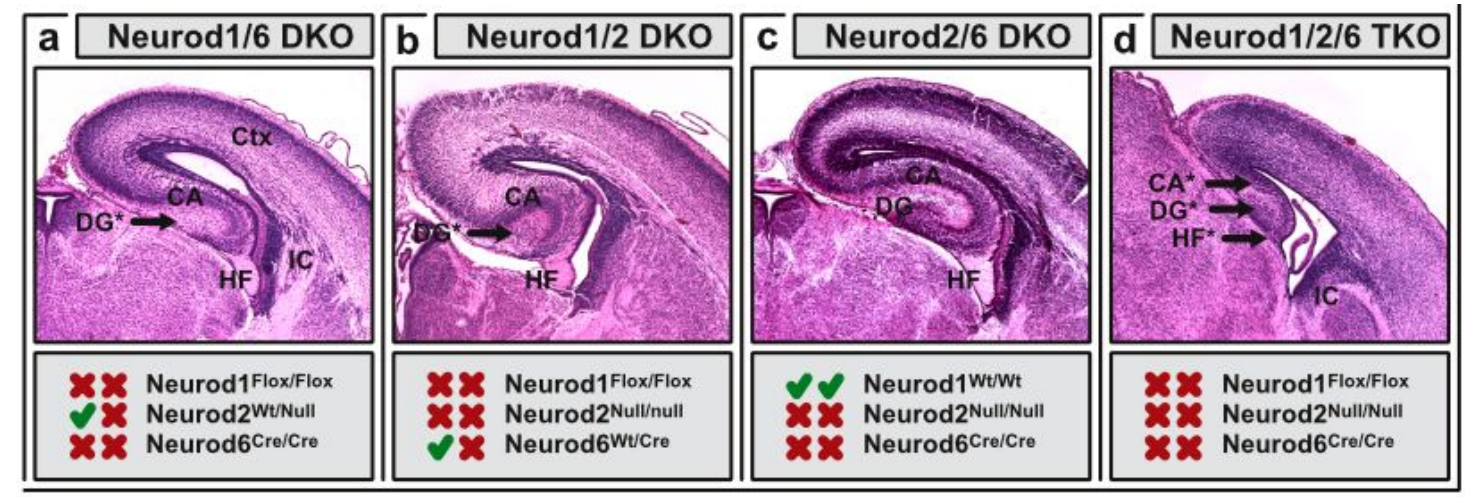

Figure 34: Hippocampal hypoplasia in Neurod1/2/6 triple-deficient mice

HE staining on coronal paraffin sections of newborn mice. (a, b) The DG was absent in Neurod1/6 doubledeficient (a) and Neurod1/2 double-deficient (b) brains. Other hippocampal structures were not severely affected in these animals. The structural phenotype resembled that of Neurod1 single mutants (sect 1.5.1). (c) Neurod2/6 double-deficient mice did not show obvious hippocampal abnormalities (sect 2.4.1). (d) The hippocampus of Neurod1/2/6 triple-deficient animals was severely hypoplastic. CA, HF and DG were not identifiable in histological sections. Asterisks denote structures that were mostly absent in mice of the corresponding genotype.

\subsubsection{Hippocampus}

The hippocampus is the most caudomedial aspect of the cerebral cortex (sect 1.2.2) and a part of the limbic system. It is involved in the consolidation of short-term to long-term memory, the formation of declarative and episodic memories, flexible cognition and social behavior. ${ }^{1}$ The hippocampus constitutes the three-layered archicortex, which is evolutionarily older than the six-layered neocortex. ${ }^{2}$

\subsubsection{Granule Neuron Differentiation}

Granule neurons exist in the archicortex, but not in the neocortex. They give rise to the hippocampal DG, which relays neocortical input to hippocampal pyramidal neurons. ${ }^{3}$ Granule neurons do robustly express Neurod 1 during embryogenesis (fig 8c, d). The onset of Neurod2 expression is later, around birth (fig 9c,d). Neurod6 is transiently expressed in a subset of relatively late born granule neurons located in the outer layers of the postnatal $\mathrm{DG}^{4}$ (fig $9 \mathrm{~g}, \mathrm{~h}$ ). Hippocampal

\footnotetext{
${ }^{1}$ Squire 1992; Rubin et al. 2014

${ }^{2}$ Hippocampus and Neocortex are thought to be complementary interconnected systems that provide unstructured short-term working memory and semantic long-term storage memory, respectively (Rolls 2013)

${ }^{3}$ Neocortical information to the hippocampus is relayed in the parahippocampal gyrus and entorhinal cortex. Entorhinal afferents feed to pyramidal neurons in the CA, either directly or indirectly via granule neurons in the DG. Granule neurons project to CA3 and provide feedforward inhibition via local interneurons. The latter is speculated to protect the hippocampus from generating false associations, such as delusions during psychosis (Bull 2011).

${ }^{4}$ Shimizu et al. 1995, fig 6; Goebbels 2002, fig 12A-C, 17F-H, tab 1; Goebbels et al. 2006, fig 4
} 
and neocortical interneurons are not generated locally. The immigrate from the ventral telencephalon and do not express Neurod $1 / 2 / 6^{1}$ (sect 1.2).

In our Neurod 1 deficient mice, granule neuron precursors were generated in the hippocampal VZ and correctly invaded the DG anlage (fig 35b, e). However, most migrating granule cells fail to undergo terminal neuron differentiation and instead died from apoptosis ${ }^{2}$ (DG* in fig 40b). As a result, the DG was almost absent in Neurod $1 / 6$ double-deficient, ${ }^{3}$ Neurod $1 / 2$ double-deficient and Neurod 1/2/6 triple-deficient mice (DG* in fig 34a, b, d, respectively). The simultaneous inactivation of only Neurod2/6 did not severely affect granule neuron differentiation or the structural development of the DG (fig 34c, fig 35c, f).

Note, that the cited publications and my initial experiments are based on conventional Neurod1 single-deficient mice (Neurod1-LacZ). The data shown in this work was obtained from conditional Neurod 1 deficient animals (Neurod1Flox $\times$ Neurod6-Cre). Both mouse models can be regarded as phenotypically identical during embryonic cortex development, but not after birth. Most postnatally born granule neurons are not part of the Neurod6 lineage. ${ }^{4}$

\subsubsection{Pyramidal Neuron Differentiation}

Hippocampal pyramidal neurons are located in a single densely packed layer, the CA, which morphologically is a continuation of the neocortical CP. The initial differentiation programs of hippocampal and neocortical pyramidal neurons are relatively similar. Neurod 1 is transiently expressed in the hippocampal and neocortical SVZ but mostly absent from mature neurons in the prenatal CA and CP (fig 8b, c). Neurod 2 and Neurod6 are expressed by immature and mature pyramidal neurons located in the hippocampal and neocortical SVZ, IZ and $\mathrm{CA} / \mathrm{CP}$ (fig 9b, c, f, g).

The hippocampal CA developed relatively normal in Neurod 1/6, Neurod $1 / 2$ and Neurod2/6 double-deficient mice (CA in fig 34a-c), but was severely aplastic in newborn triple-deficient animals (arrows in fig 33; CA* in fig 34d). The expression of either Neurod1, Neurod2 or Neurod6 is thus sufficient to induce hippocampal pyramidal neuron differentiation. The hippocampal sulcus, MZ and VZ are normally devoid of Neurod 1/2/6 positive or Neurod6-lineage cells. These areas were subsequently not severely affected by the combined loss of Neurol/2/6 (fig 35c).

In contrast to triple-deficient pyramidal neurons in the lateral neocortex, which were able to differentiate and migrate into the CP (sect 2.5.4.3), all triple-deficient pyramidal cells in the archicortical hippocampus were lost at the neuronal precursor stage, well before the initiation of terminal differentiation and radial

\footnotetext{
${ }^{1}$ Neurod1/2/6 expression was never reported in the ventral telencephalon. Neurod1: Schwab et al. 1998, fig 7E, 8G; Miyata et al. 1999, fig 5g; Liu et al. 2000, fig 1d. Neurod2: Schwab et al. 1998, fig 7F, 8H; Olson et al. 2001, fig 3E, F; Lin et al. 2004, fig 4D. Neurod6: Schwab et al. 1998, fig 7D, 8F; Goebbels 2002, fig 16, 17; Goebbels et al. 2006, fig 5.

${ }^{2}$ Liu et al. 2000; Miyata et al. 1999; Schwab et al. 2000

${ }^{3}$ Neurod 1/6 double-deficient animals have already been described in Schwab et al. 2000

${ }^{4}$ Goebbels et al. 2006, fig 4a; Goebbels 2002, fig 12d-f, fig 17f-h

The DG is not completely lost but strongly hypoplastic (estimated loss of granule neurons: $90 \%$ in adult conditional Neurod 1 deficient (Neurod1-Flox $\times$ Neurod6-Cre) mice (not shown).
} 


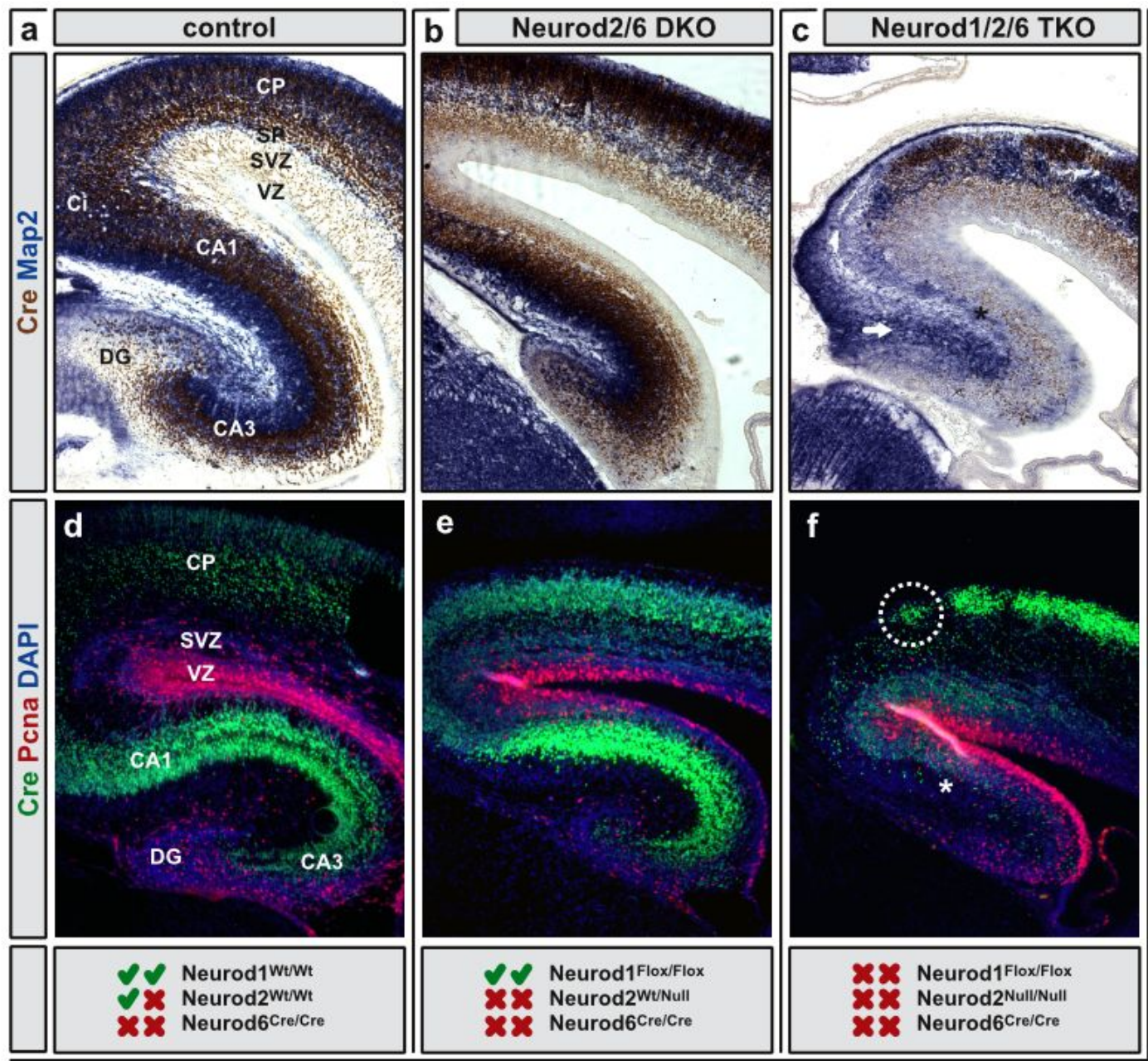

Figure 35: Loss of hippocampal pyramidal neurons

Paraffin sections of newborn control (a,d), Neurod2/6 double-deficient (b,e) and Neurod1/2/6 tripledeficient (c, f) mice. (a-c) Chromogenic IHC for Cre (brown) and Map2 (blue). (d-f) Immunofluorescence for Cre (green) and Pcna (red) in combination with nuclear DAPI staining (blue). Asterisks mark the absence of Neurod6-Cre positive pyramidal neurons in the Neurod1/2/6 triple-deficient CA.

migration into the AC (fig 35c). Strong mitotic activity in the hippocampal VZ and Cre expression in the adjacent SVZ suggested that the number of pyramidal neuron precursor cells that had been born and that had started to migrate radially was not decreased. However, early migrating cells apparently failed to differentiate and instead died from apoptosis, shortly after the expected onset of Neurod2/6 expression. The hippocampus of newborn Neurod 1/2/6 tripledeficient animals was almost devoid of clearly Neurod6-Cre positive pyramidal neurons, and it lacked the typical cell-dense CA (fig 35c, f). The complete loss of hippocampal projection neurons resulted in absence of the associated fiber tracts, $\mathrm{HF}$ and $\mathrm{HC}$ (fig 33).

\subsubsection{Neocortex}

The gray matter of the neocortex was not uniformly affected in newborn Neurod1/2/6 triple-deficient mice. Very rostrolateral areas had developed 


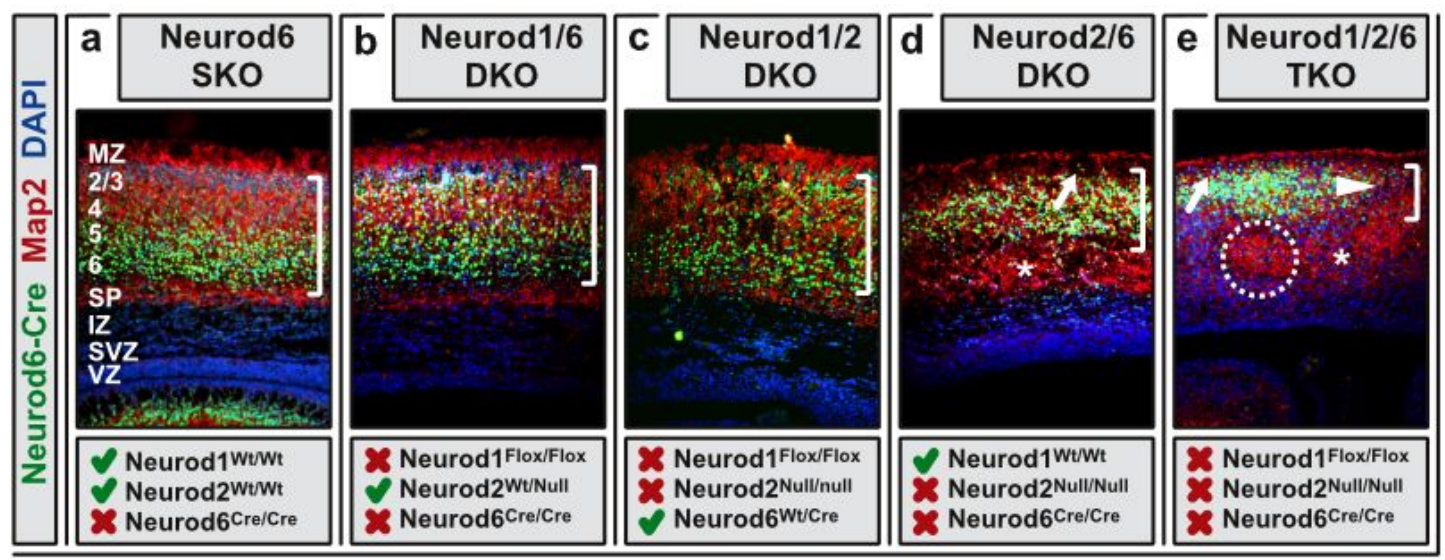

Figure 36: Cortex development in the absence of NeuroD genes

Coronal paraffin sections from the neocortex of newborn mice. Combined IHC for Cre (green) and Map2 (red) and DAPI based nuclear counterstaining (blue). Cre marks putatively Neurod6 expressing cells (all mice carry the Neurod6 allele). Map2 is selectively expressed by mature neurons and identifies the SP and the lower border of the CP. (a) Neurod6 single-deficient mice resembled the wild type situation. (b) Neurod1/6 double-deficient animals did not exhibit obvious structural abnormalities. Neurod6-Cre signals from the upper cortical layers, however, were mildly increased. (c) Neurod1/2 double-deficient mice showed mild migratory abnormalities of Neurod6-Cre positive pyramidal neurons. The thickness of the CP was not changed. A subset of strongly Neurod6-Cre expressing cells was situated within the SP resulting in a weakly defined SP/CP boundary. (d) The previously described Neurod2/6 double-deficient mice showed clear migratory abnormalities. Subpopulations of Neurod6-Cre positive neurons had failed to migrate to the CP or had over-migrated into the MZ. The thickness of the CP was moderately reduced. (e) Neurod1/2/6 triple-deficient mice were strongest affected. Different populations of Neurod6-Cre positive and Neurod6-Cre negative cells had aggregated and formed cell-dens clusters (dotted lines). Brackets mark the cortical area containing strongly Neurod6-Cre positive pyramidal neurons. Arrows point to ectopically localized Neurod6-Cre positive cells in the MZ. Asterisks denote areas in the deeper CP that are Map2-positive but lack Neurod6-Cre expression in Neurod2/6 double- and Neurod1/2/6 triple-deficient mice.

relatively normal, while central and caudomedial regions were disorganized and substantially thinner (fig 33c, f). However, even in the strongest affected parahippocampal areas, large numbers of neocortical pyramidal neurons were able to survive, differentiate and migrate into the CP. Most of the neocortical abnormalities already described in Neurod2/6 double-deficient mice (sect 2.4.2) were exacerbated upon additional inactivation of Neurod 1 :

The differentiation and radial migration of surviving pyramidal neurons was stronger disturbed in Neurod 1/2/6 triple-deficient mice than in Neurod $2 / 6$ double-deficient animals (sect 2.4.2; fig 33b, c; fig 36d, e). The cerebral cortex of newborn triple-deficient pups was smaller and the typical columnar microarchitecture was lost in most regions (fig 36e; fig 37c). Pyramidal neurons instead had formed large aggregates that were separated by Map2-positive septa of low cellular density (arrow heads in fig 36e; fig 37b, c). These abnormal structures might correspond to enlarged cortical columns and dendritic brushes, respectively (sect 2.4.3).

The radial distribution of strongly Neurod6-Cre expressing pyramidal neurons within the CP was increasingly altered in Neurod $1 / 6$ double-deficient, Neurod $1 / 2$ double-deficient, Neurod2/6 double-deficient, and Neurod 1/2/6 triple-deficient mice (fig 36). It is not clear, to what extend that effect was caused by abnormal radial migration or changes in Neurod6-promoter activity. 


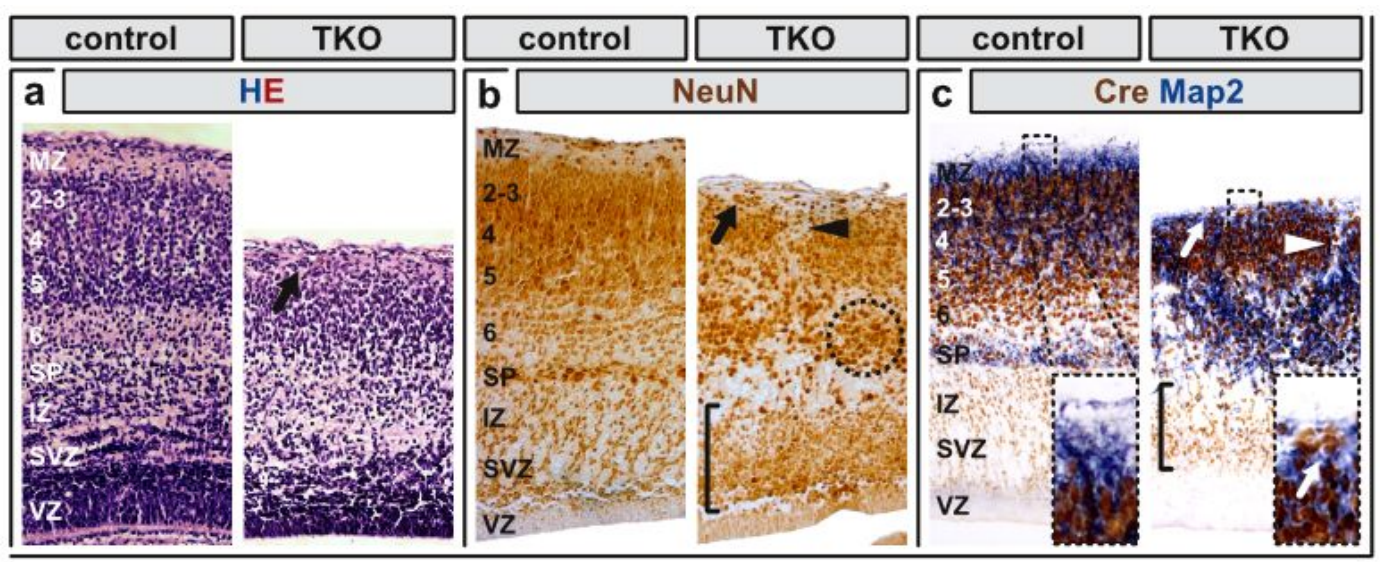

Figure 37: Cortical plate formation in Neurod1/2/6 triple-deficient mice

Paraffin sections from the neocortex of newborn Neurod1/2/6 triple-deficient mice (TKO) and control (Neurod1/6 double-deficient) littermates. (a) HE overview staining. The thickness of the neocortex was reduced and the typical columnar micro-architecture was lost. (b) IHC for the pan-neuronally expressed RNA-binding protein NeuN (brown) confirmed basic neuronal differentiation of neocortical Neurod1/2/6 tripledeficient pyramidal cells. (c) Combined IHC for Cre recombinase expressed under the control of the Neurod6 promoter (brown), and the dendritic marker Map2 (blue). In newborn Neurod1/2/6 triple-deficient mice, a subset of the differentiated neurons had accumulated in the SVZ/IZ (brackets). The neocortical SP was hardly identifiable and SP neurons seemed dispersed. Most pyramidal neurons had migrated into the CP but the columnar substructure of the neocortex had been lost and replaced by irregular aggregates (dotted circle) that were accompanied by regions of reduced cellular density (arrow heads). Many pyramidal neurons had over-migrated and were ectopically localized in the MZ (arrows).

\subsubsection{Radial Migration and Laminarization}

The portion of neuronal cells that had failed to migrate radially and that instead had accumulated in the SVZ was further increased in newborn Neurod 1/2/6 triple-deficient mice (brackets in fig 37b, c; fig 35f) when compared to the already described Neurod2/6 double-deficient animals (fig 20b, d; fig 35e). However, even in stronger affected caudomedial cortex areas a large number of pyramidal neurons could differentiate in the absence of all three factors and was able to migrate radially into the $\mathrm{CP}$ (fig 37b, c; fig 38b; fig 39b).

As in Neurod2/6 double-deficient animals (sect 2.4.2.2), a subset of migrating triple-deficient pyramidal neurons failed to settle in the CP and instead invaded the MZ, which is normally devoid of Neurod6-lineage cells (arrows in fig 37; fig 20). In some cortex areas, the MZ was densely populated with Neurod6-Cre positive pyramidal neurons (fig 37c) and did not delineate from the CP.

The tangential layering of the CP was hardly detectable in newborn Neurod 1/2/6 triple-deficient mice (fig 38; fig 39). Subpopulations of neocortical pyramidal neurons had formed irregular focal aggregations (dotted circles in fig 37b; fig 39b). In contrast to Neurod2/6 double-deficient animals, where neocortical laminarization and the identity of neocortical pyramidal neurons had not been found severely changed (sect 2.4.2.4; sect 2.4.2.5), Neurod $1 / 2 / 6$ triple-deficient mice showed little signs of terminal pyramidal neuron differentiation and most pyramidal neurons failed to acquire layer specific neuronal identities (sect 2.5.4.3). 


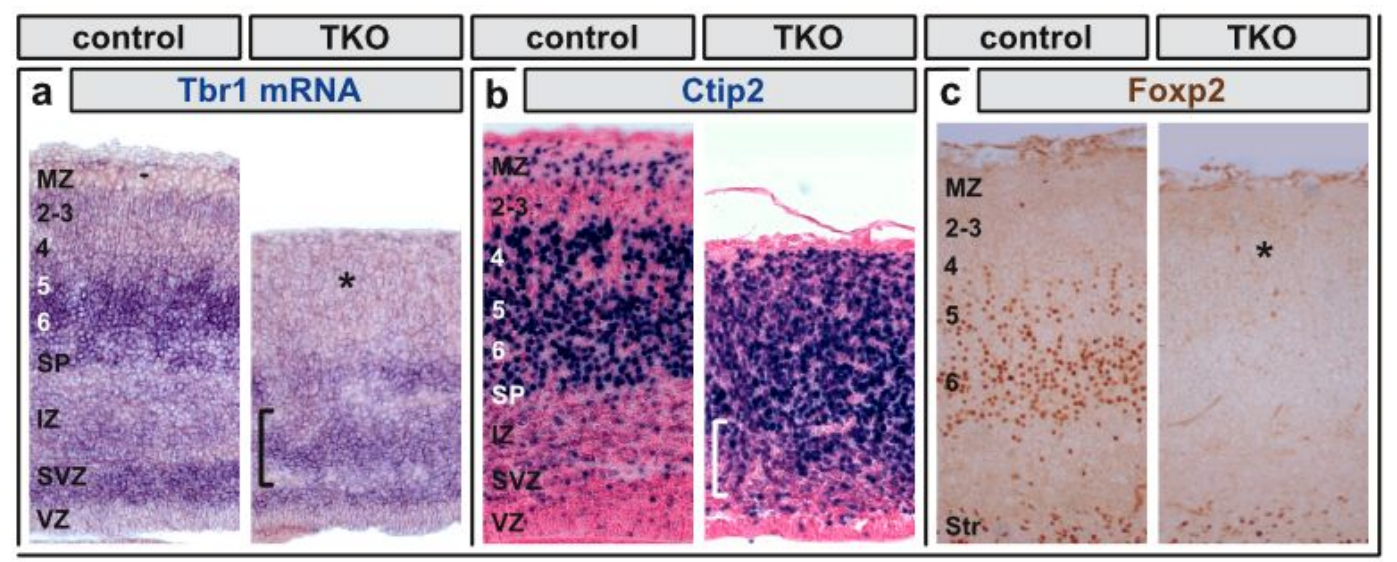

Figure 38: Deeper layer identity in Neurod1/2/6 triple-deficient mice

Paraffin sections from the neocortex of newborn Neurod1/2/6 triple-deficient mice (TKO) and control (Neurod1/6 double-deficient) littermates. (a) ISH for Tbr1 (blue) confirmed the accumulation of basically differentiated neurons in the SVZ/IZ. (b) IHC for Ctip2 (blue, counterstained in red), a transcription factor predominantly expressed by subcortically projecting pyramidal neurons (and inhibitory interneurons), revealed a broad expansion of the evolutionary old motoneuron identity. (c) IHC for Foxp2 (brown), a marker of corticothalamic projecting pyramidal neurons was negative in the neocortex. Absence of Tbr1 and Foxp2 from the CP suggests loss or misspecification of layer 6 neurons. Asterisks mark regions where the tested protein was strongly expressed in controls and nearly absent in Neurod1/2/6 triple-deficient mice. Brackets mark accumulations of neuronal cells in the SVZ/IZ.

\subsubsection{Determination and Early Differentiation}

Tbr1 and Brn2 are two transcription factors involved in the determination and differentiation of cortical pyramidal neurons (sect 2.4.2.1). In newborn Neurod1/2/6 triple-deficient mice, both genes were expressed by immature neurons of the SVZ and IZ, but mostly absent from the second expression domains in layer 6 and layers $2-5$ of the CP (fig 38a and fig 39a versus fig 20 and fig 22a).

Tbr1 represses Fezf2 and Ctip2 in corticothalamic projection neurons located in layer 6. ${ }^{1}$ Brn1/2 activate Satb2 that in turn represses Ctip2 in callosal projection neurons, which are predominantly located in layer $2 / 3$ and to a lesser extend layer $5 / 6$ (sect 2.4.2.4). ${ }^{2}$ As a result, Ctip2 is normally predominantly expressed in layer 5/6 where it is involved in the acquisition of the corticofugal identity and the establishment of corticospinal connectivity (sect 2.4.2.5).

In newborn Neurod 1/2/6 triple-deficient mice, the Ctip2 expression domain was expanded. Especially in the lateral cortex, ${ }^{3} \mathrm{Ctip} 2$ was robustly expressed by pyramidal neurons of all cortical layers (fig 38b). The expression of Satb2, which is a determinant of callosal projection neurons and normally enriched in upper layers, was reduced in Neurod 1/2/6 triple-deficient mice. Cellular Satb2 protein levels in the CP were roughly comparable to those of immature neurons located

\footnotetext{
${ }^{1}$ McKenna et al. 2011

${ }^{2}$ Dominguez et al. 2013

${ }^{3}$ The Ctip2 expression domain was assessed in the lateral neocortex because central cortical areas of newborn Neurod $1 / 2 / 6$ triple-deficient mice were dominated by focal congregations of pyramidal neurons that masked the laminar organization and may have formed secondarily due to abnormal neurite outgrowth and/or tangential migration (fig 35c, f; fig 43d').
} 


\begin{tabular}{|c|c|c|c|c|c|}
\hline control & TKO & control & TKO & control & TKO \\
\hline \multicolumn{2}{|c|}{ Brn2 } & \multicolumn{2}{|c|}{ Satb2 } & \multicolumn{2}{|c|}{ Lmo4 } \\
\hline $\begin{array}{l}\text { MZ } \\
2-3 \\
4 \\
5 \\
6 \\
\text { SP } \\
\text { IZ } \\
\text { SVz } \\
\text { VZ }\end{array}$ & & 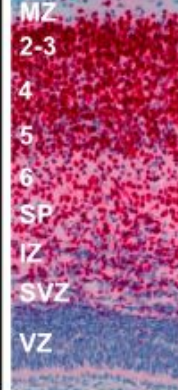 & 8 & $\begin{array}{l}M z \\
2-3 \\
4 \\
5 \\
5 \\
6 \\
S R \\
1 z \\
s v z \\
V z\end{array}$ & \\
\hline
\end{tabular}

Figure 39: Upper layer identity in Neurod1/2/6 triple-deficient mice

Paraffin sections from the neocortex of newborn Neurod1/2/6 triple-deficient mice (TKO) and control (Neurod1/6 double-deficient) littermates. (a) ISH for Brn2 (brown) confirmed the accumulation of basically differentiated neurons in the SVZ/IZ. (b) IHC for Satb2 (red, counterstained in blue), a transcription factor expressed by immature pyramidal cells and mature corticocortically projecting pyramidal neurons. Satb2 and Ctip2 normally follow mutually exclusive expression pattern. Broad expression of both proteins in the CP suggested, that the corresponding identities did not properly separate in Neurod1/2/6 triple-deficient mice. Pyramidal neurons in the CP might have failed to differentiation terminally after reaching a basic pyramidal neuron identity characterized by the expression of Ctip2 and Satb2. (c) IHC for Lmo4 (blue, counterstained in red), a transcription factor expressed by most terminally differentiated pyramidal neurons of the motor- but not the somatosensory cortex. Asterisks mark regions where the tested protein was strongly expressed in controls and nearly absent in Neurod1/2/6 triple-deficient mice. The dotted circle marks abnormal cellular aggregations in the Neurod1/2/6 triple-deficient cortex. Brackets mark accumulations of neuronal cells in the SVZ/IZ.

in the SVZ and IZ of control animals (fig 39b). The surviving pyramidal neurons in the $\mathrm{CP}$ of Neurod1/2/6 triple-deficient mice can thus be expected to have gone through normal neuronal determination and at least basic pyramidal neuronal differentiation.

\subsubsection{Terminal Differentiation and Identity}

Foxp2 is a transcription factor normally expressed by terminally differentiated pyramidal neurons, which are located in layer 6 of the neocortex and project to the thalamus ${ }^{1}$ (sect 2.4.2.5). Foxp2 was completely absent from the neocortex of Neurod 1/2/6 triple-deficient mice (fig 38c). Together with absent Tbr 1 expression in the CP (fig 38a), this suggests that the molecular identity of layer 6 is lost in Neurod $1 / 2 / 6$ triple-deficient mice.

Lmo4, which is normally enriched in upper layers of the lateral cortex ${ }^{2}$ (sect 2.4.6), was hardly detectable in the neocortex of newborn Neurod $1 / 2 / 6$ triple-deficient mice (fig 39c). Remaining Lmo4 expression was localized towards the outer surface of the neocortex, which suggests that the CP principally developed following the typical inside-first outside-last manner (sect 1.2.3). Due to low

\footnotetext{
${ }^{1}$ Ferland et al. 2003, fig 2, tab. 1; Takahashi et al. 2008, fig 3b

${ }^{2}$ Sun et al. 2005, fig 3: ISH for Lmo4 mRNA in whole mount (D) and sagittally sectioned (I) brains of $\mathrm{P} 1$ mice
} 


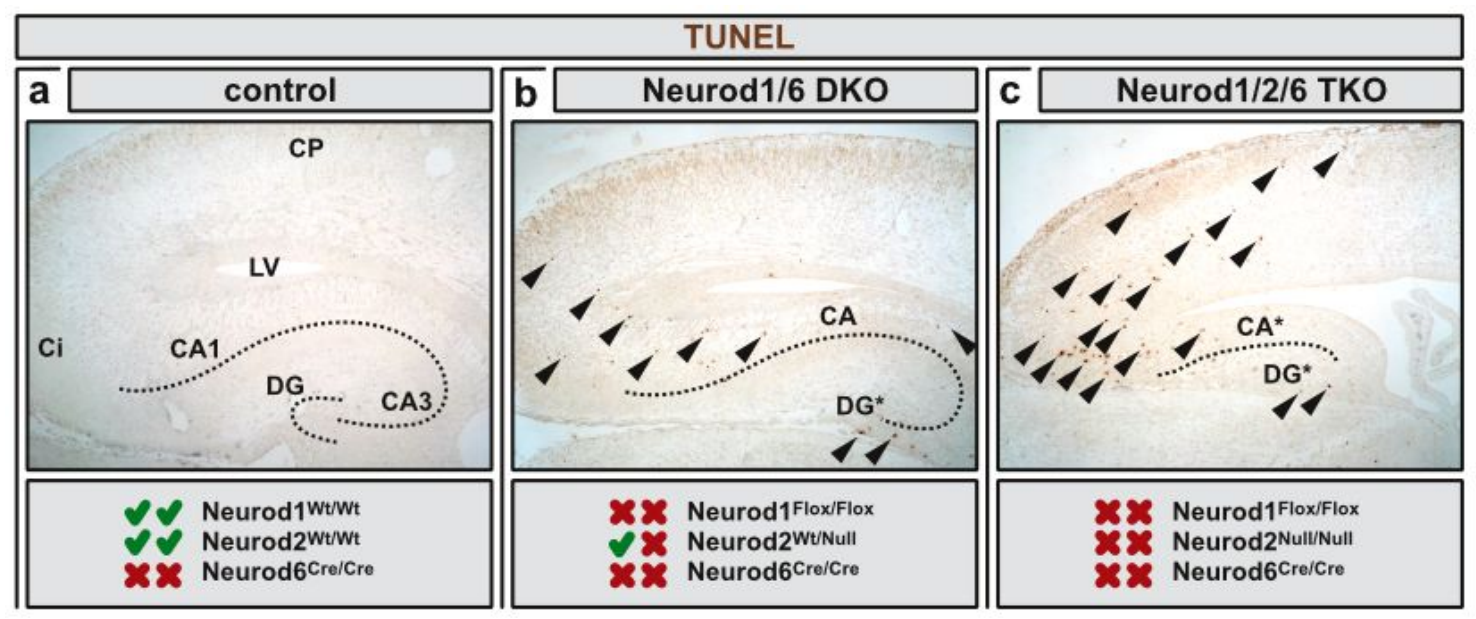

Figure 40: Apoptosis in Neurod1/2/6 triple-deficient mice

TUNEL staining (brown) to visualize apoptotic cells (arrow heads) in paraffin sections of newborn mice. (a) Hardly any clearly TUNEL-positive cells could be identified in Neurod6 single-deficient control brains. (b) Apoptotic cell death was significantly increased in the Ci and CA1 of Neurod1/6 double-deficient animals harboring only one allele of Neurod2. (c) The strongest apoptosis rates were observed in the caudomedial cortex of Neurod1/2/6 triple-deficient mice. Loss of DG granule neurons (DG* in b, c) can be attributed to the inactivation of Neurod1 alone (sect 1.5.1). Asterisks denote hypoplastic structures.

the expression levels of Lmo4, it was not possible to reliably evaluate cortical arealization in Neurod 1/2/6 triple-deficient mice.

\subsubsection{Developmental Cell Death}

Terminal deoxynucleotidyl transferase dUTP nick end labeling (TUNEL) reactions in cortical brain sections confirmed massively increased developmental cell death in the cortex of newborn Neurod $1 / 2 / 6$ triple-deficient mice (fig 40c) and of Neurod $1 / 6$ double-deficient animals that carried only one allele of Neurod2 (fig 40b). Most TUNEL-positive cells were located in the caudomedial neocortex and the cingulum. Apoptosis rates in the Neurod 1/2/6 triple-deficient hippocampus were comparably low (CA* in fig $40 \mathrm{c}$ ). The small size of the hippocampal formation and absence of the CA at birth suggested, that most hippocampal pyramidal neurons had already died during embryogenesis. The hippocampal VZ showed normal mitotic activity at birth (fig 35f). However, the generation of pyramidal neurons has normally already completed at E19 and the VZ instead generates glia cells, ${ }^{1}$ which are not part of the Neurod6-linage and generally Neurod1/2/6-negative. A detailed quantitative analysis of embryonic brain development is to be performed but was beyond the scope of this work.

Cell death of hippocampal granule neurons and the absence of the DG in doubleand triple-deficient animals (DG* in fig $40 \mathrm{~b}, \mathrm{c}$ ) can be explained by the inactivation of Neurod 1 alone (sect 1.5.1).

\footnotetext{
${ }^{1}$ Reznikov 1991, sect 1.2.1, "Neurogenesis and Gliogenesis in the Hippocampus", p 7
} 


\subsubsection{Cortical Connectivity}

The connectivity of the cerebral cortex, which had already been strongly affected in Neurod2/6 double-deficient mice (sect 2.4.4), was further reduced upon additional inactivation of Neurod 1 (fig $42 \mathrm{~b}$ ). The relatively small number of remaining fibers projected mostly intrahemispherically (fig 41). Only few cortical axons were able to leave the pallium (fig 41d), those mainly targeted the spinal cord (fig 42a). The number of afferent projections reaching the neocortex was also strongly reduced (fig $43 \mathrm{~d}$ ), which is probably a secondary effect resulting from defective corticofugal connectivity (sect 2.5.6.3).

\subsubsection{Intracortical Projections}

Intrahemispheric fiber tracts were strongly reduced in the absence of Neurod 1/2/6. Bidirectional axon tracing using the hydrophobic cyanine dye DiI, labeled only few dispersed long-range projections in fixed brains of newborn triple-deficient mice (fig 41a). Fluorescent IHC for the axonal cell adhesion molecule L1cam, identified remaining axon bundles. The relative focus of L1cam immunofluorescence was shifted from the caudal to the rostral neocortex (arrows in fig 41b).

The prominent upregulation of Robo 1 in the CP of Neurod2/6 double-deficient mice (sect 2.4.4.5) was lost upon the additional inactivation of Neurod 1 (asterisk in fig 41c). In newborn triple-deficient animals, Robol was expressed at approximately normal levels. Robol IHC identified fasciculated axon bundles in the rostroventral IC and in non-crossing aspects of the CC (double arrow in fig 41c).

The above described axonal Cre-reporter allele (sect 2.4.4.6) was used to selectively visualize axons originating from Neurod6-lineage pyramidal neurons. In control brains, all cortical projection tracts (CC, AC, HC, HF, IC, LOT) were strongly GFP-positive, and the CP was scattered with fluorescently labeled axons ${ }^{1}$ (left panel in fig 41d). In newborn Neurod 1/2/6 triple-deficient brains, normal neocortical and archicortical projections were hardly identifiable. Instead, abnormal fasciculated fiber bundles appeared to follow random trajectories within the neocortex (right panel in fig 41d). The diffuse fluorescent labeling of the CP was not significantly reduced, suggesting the presence of short or unfasciculated axons originating from remaining neocortical Neurod $1 / 2 / 6$ triple-deficient pyramidal neurons. The lateral olfactory tract (LOT), which originates from mitral and tufted cells of the paleocortical OB, was reduced but identifiable in tripledeficient mice (fig $41 \mathrm{~d}$ and data not shown). LacZ histochemistry confirmed that endogenous Mapt promoter of the Cre-reporter construct was principally active in all areas and layers of the triple-deficient neocortex (fig $41 \mathrm{~d}$ ). ${ }^{2}$

\footnotetext{
${ }^{1}$ The control animal in fig $41 \mathrm{~d}$ has the genotype Neurod $1^{\text {Flox } / \text { Flox }} \times$ Neurod $2^{\text {Wt/Null }} \times$ Neurod6 $6^{\text {Cre/Cre }}$. These mice showed delayed midline fusion, incomplete formation of the CC and PBs.

${ }^{2}$ Limitations apply as elaborated in sect 2.4.4.6 (footnote). It should be emphasized that the layering of the CP was severely disturbed and that many pyramidal neurons were weakly or not lacZ-positive in newborn Neurod 1/2/6 triple-deficient mice.
} 


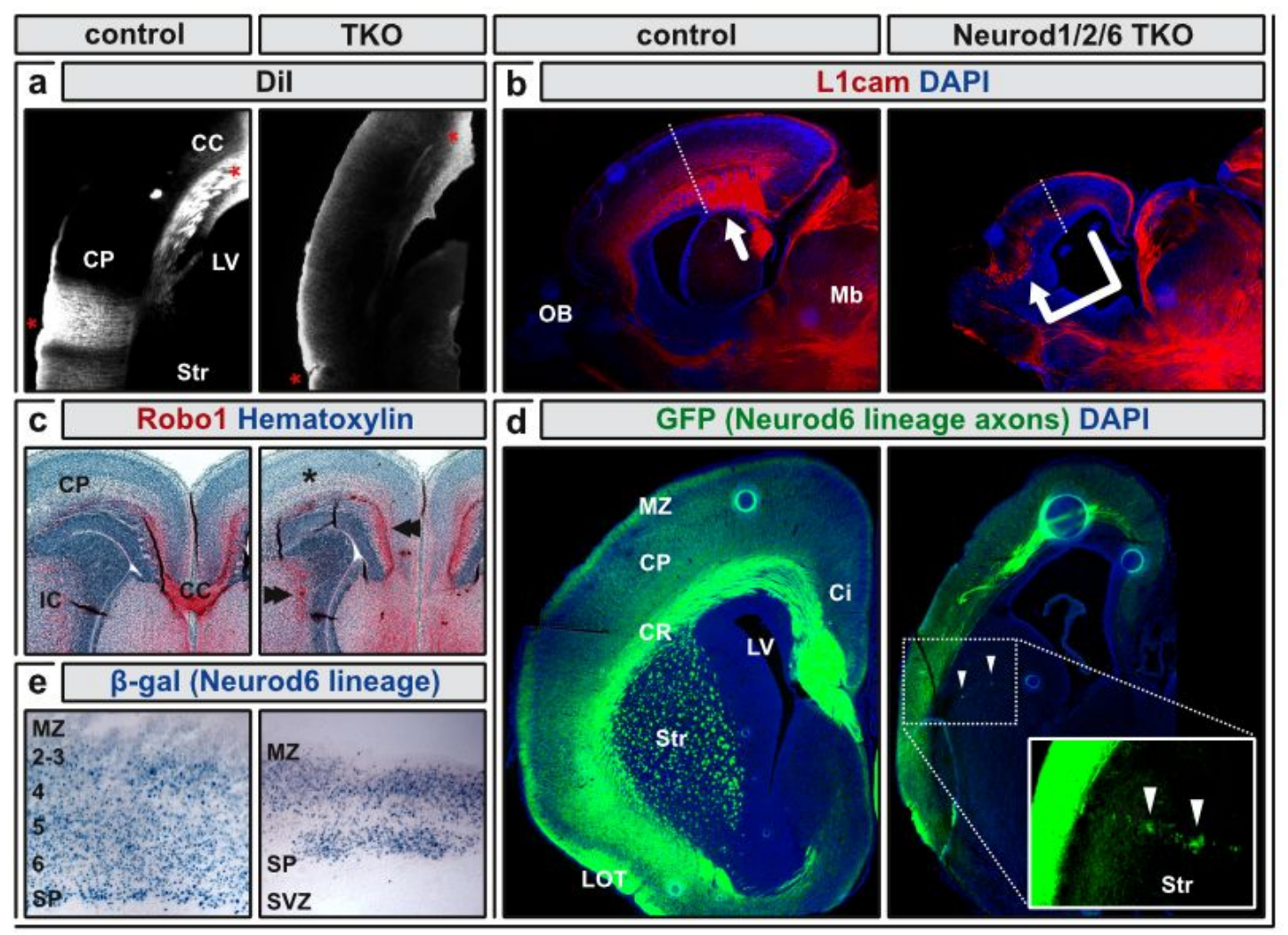

Figure 41: Intracortical connectivity in Neurod1/2/6 triple-deficient mice

(a) Dil crystals (red asterisks) were placed in the central CP and the paramedial CC of formalin-fixed brains from newborn mice. Dil molecules are very lipophilic and avoid intra- and intercellular fluids but readily diffuse along lipid membranes. The incubation of injected brains for several weeks in an aqueous solution resulted in bidirectional labeling of axons running in close proximity of Dil injection sites. Control tissue showed strong fluorescence in the $\mathrm{CP}, \mathrm{CR}$ and $\mathrm{CC}$. These structures were virtually devoid of specific signal in triple-deficient brains, suggesting that neocortical axon growth is severely affected in the absence of Neurod1/2/6. (b) IF for the axonal surface protein L1cam (red) revealed the neocortical axonal compartment to be strongly reduced but not absent in parasagittal sections of newborn Neurod1/2/6 triple-deficient mice. In controls, the majority of L1cam-positive axons were located in the caudal neocortex, mostly contributing to the CC. In tripledeficient mice, the number of L1cam-positive axons was severely reduced in the caudal half but only mildly reduced in the rostral half of the neocortex. The relative focus of axonal connectivity had thus been shifted rostrally (arrows). The dotted line represents the rostrocaudal center of the neocortex. (c) Chromogenic IHC for Robo1 (red) in horizontal paraffin sections of newborn mice identified the rostral IC in Neurod1/2/6 triple-deficient brains (double arrows). Robo1-upregulation in the CP (sect 2.4.4.5a) was not observed. (d) The previously described bicistronic Cre-reporter allele (sect 2.4.4.6) was used to fluorescently label axons originating from Neurod6-Cre positive cortical pyramidal neurons. Newborn Neurod1/6 double-deficient mice carrying only one allele of Neurod2 showed dense axonal networks in the CC, IC, CR and lower CP. The number of labeled cortical axons was strongly reduced in the Neurod1/2/6 triple-deficient littermate (the integrated fluorescent density was reduced by $80 \%$ ). Corticofugal projections (arrow heads) were hardly visible, but definitely present (the inset shows an intentionally overexposed image from the boxed region of a more caudal section). (e) LacZ staining showed that the endogenous Mapt-promoter driving the bicistronic reporter allele was transcriptionally active in pyramidal neurons of the triple-deficient neocortex.

\subsubsection{Subcortical Projections}

In newborn control mice, a large number of fluorescently labeled corticofugal fiber tracts had penetrated the striatum to form the IC and CST (fig 41d). In Neurod 1/2/6 triple-deficient animals, the striatum was nearly devoid of fluorescent signals. However, a thin band of GFP-positive axons formed a 


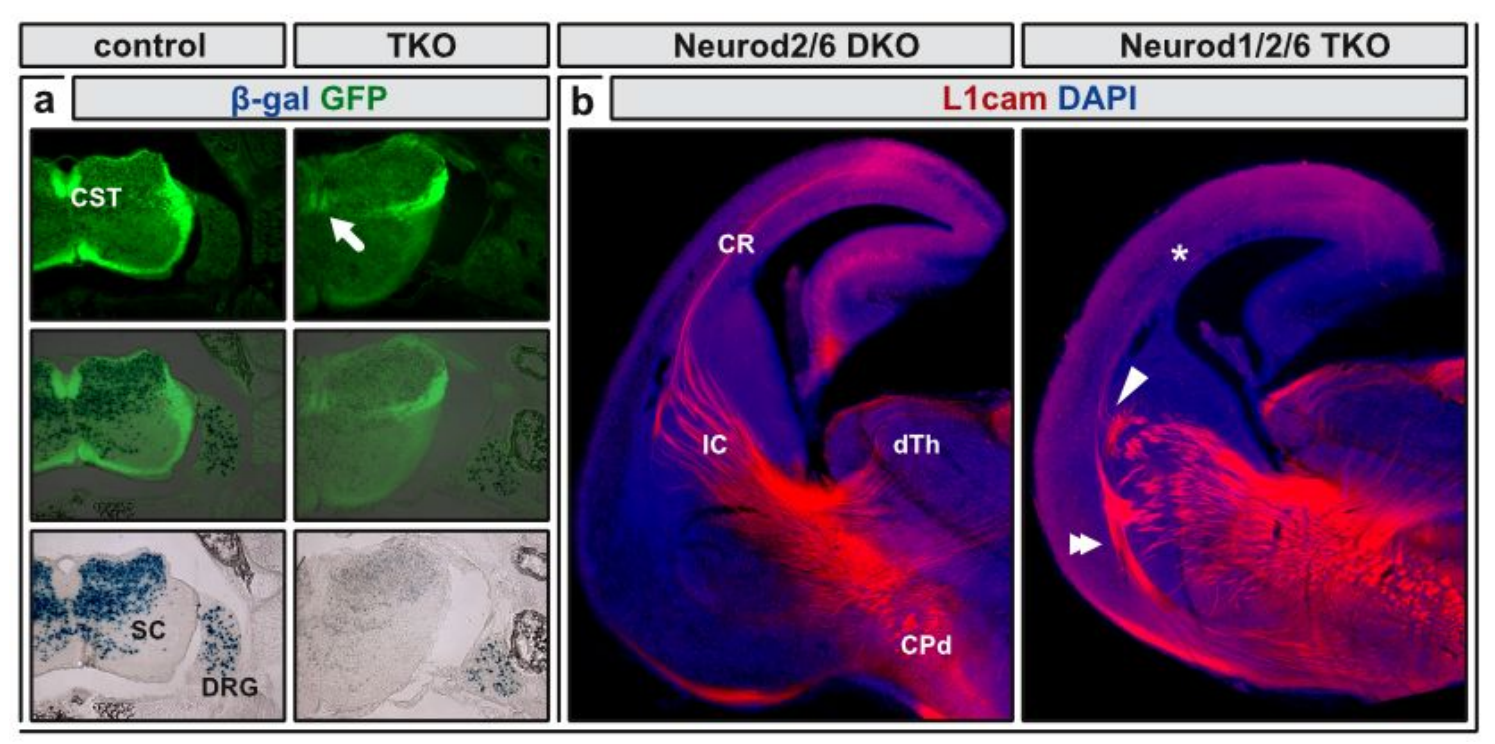

Figure 42: Subcortical connectivity in Neurod1/2/6 triple-deficient mice

(a) The described Cre-reporter allele (sect 2.4.4.6) was used to label axons (green) and cell bodies (blue) of the Neurod6-lineage in the SC of newborn mice. The CST (arrow) could be identified in control and Neurod1/2/6 triple-deficient mice at various spinal levels. The reporter was active in neocortical pyramidal neurons (fig 42e) and afferent DRG projections neurons, but not in spinal Neurod6-lineage interneurons. (b) IF for (red) in coronal brain sections identified a large number of axons in the IC, TCT and CPd of Neurod1/2/6 triple-deficient mice. The PSPB was only penetrated by very few L1cam-positive axons in these sections (arrow head). The presence of a ventrally oriented aberrant fiber bundle (double arrow) suggests that corticospinal axons had followed an abnormal trajectory to eventually reach the CPd and SC.

rudimentary CST that could only be identified in a few mid-coronal brain sections (arrow heads in fig 41d).

In principle, Neurod 1/2/6 triple-deficient motoneurons were able to form long range projections that could reach the spinal cord (SC). The dorsal CST of newborn triple-deficient mice was weakly GFP-positive and the CST cross section area was strongly reduced (arrow fig 42a). ${ }^{1}$ Because the Mapt-promoter driven reporter allele was clearly active in neocortical motoneurons (fig 41e), low GFP signal intensity in the spinal CST most probably results from a very low number of corticospinal axons.

Noteworthy, the Mapt-promoter of the Cre-reporter allele was not universally active. Expression was robust in in triple-deficient sensory neurons located in dorsal root ganglions (DRGs), but barely detectable in peptidergic neurons located in the dorsal horn of the spinal gray matter (fig $42 \mathrm{a}) .^{2}$

${ }^{1}$ The size and signal intensity of the Neurod $1 / 2 / 6$ triple-deficient CST was not quantified because usable tissue was limited. Membrane associated GFP quickly diffused in fixed tissue sections and breeding was complicated due to Mapt and Neurod2 being located on the same chromosome (footnote in sect 2.4.4.6). The fluorescently labeled axon tract, however, was clearly identifiable at different spinal levels and the shape of the surrounding dorsal funiculus was not significantly reduced in the absence of Neurod 1/2/6 (Bröhl et al. 2008, fig 7).

${ }^{2}$ This is compatible with our previous finding that the identity of dynorphin- and galaninexpressing neurons in the dorsal horn depends on the expression of Neurod $1 / 2 / 6$ (Bröhl et al. 2008, fig 7-9) and suggests that these cells might fail to undergo terminal neuronal differentiate and do not acquire an alternative fate in the absence of Neurod1/2/6. 
Fluorescent immunostaining for L1cam in coronal brain sections identified comparable numbers of afferent projections in the IC, corticothalamic tract (CTT) and cerebral peduncle (CPd) of newborn control and Neurod 1/2/6 tripledeficient mice (fig 42b). However, afferents that crossed the PSPB and invaded the Neurod1/2/6 triple-deficient neocortex were rare (arrow in fig $42 \mathrm{~b}$ ). These afferent axons originate from subcortical neurons that are not part of the Neuod6 lineage (sect 2.1). Their inability to normally invade the neocortex can thus not be explained by cell-intrinsic defects, but is most probably caused by the absence of some attractive signal originating in the neocortex.

\subsubsection{Thalamocortical Handshake}

Most peripheral input to the cerebral cortex is synaptically relayed in the diencephalic thalamus. Thalamocortical afferents (TCAs) originate from excitatory projection neurons that are Neurod2-negative ${ }^{1}$ and (at least to the vast majority) not part of the Neurod6-lineage. ${ }^{2}$ The cell-intrinsic developmental program of thalamocortically projecting neurons is thus most probably not affected by the loss of Neurod $1 / 2 / 6 .^{3}$

Axon growth following complicated paths does generally require axonal growth cones to sequentially interact with a variety of molecular cues that are provided by tissue environments along the axonal path. ${ }^{4}$ Axons of the developing thalamocortical tract (TCT) cross the diencephalic-telencephalic boundary, turn dorsally into the ventral telencephalon, ascent along a narrow striatal corridor thereby forming the striatal IC, and reach the PSPB. All those structures do not contain cells that express any of the three NeuroD genes and thus can be assumed to provide normal environmental cues in Neurod 1/2/6 triple-deficient mice. Immunofluorescence (IF) for L1cam and non-phosphorylated medium neurofilament (Fnp7) confirmed that initial thalamocortical axon growth was not substantially altered and that the TCT could reach the PSPB in Neurod1/2/6 triple-deficient mice (arrow head in fig 42b; arrow in fig 43d).

In mice all expressing any one of the three NeuroD genes, thalamocortical axons did cross the PSPB, turned ventrally, and reached the CR of the central neocortex (fig 43a-c, a'-c'). In Neurod 1/2/6 triple-deficient mice, most thalamic axons failed to cross the PSPB and instead formed a massive ventrally oriented aberrant fiber bundle in the caudolateral cortex (arrow in fig 42d; double arrow head in fig 42b). However, a small number of axons did cross the PSPB and followed

\footnotetext{
${ }^{1}$ Olson et al. 2001, fig 3 (“ $\beta$-galactosidase staining was present in [...] a subset of thalamic and hypothalamic nuclei - detailed expression data to be presented elsewhere") and further specified in Lin et al. 2004, fig 3d, tab 1 (Neurod2-LacZ was expressed in epithalamic "habenular nuclei" but was absent from the "remainder of [the] thalamus").

${ }^{2}$ Goebbels 2002, fig 9, tab 1 and further specified in Goebbels et al. 2006, fig 2g, fig 3a+d, tab 1 (the thalamic geniculate body "displays a scattered pattern of $\beta$-galactosidase-positive [Neurod6-lineage] cells.")

${ }^{3}$ Neurod 1 is robustly expressed in some thalamic nuclei (Goebbels 2002, fig 18, top panel). The majority of thalamocortically projecting neurons, however, is not expected to be affected in Neurod1/2/6 triple-deficient mice because only a very small number of dispersed thalamic neurons is part of the Neurod6-lineage (Goebbels et al. 2006, tab 1: "dispersed $\beta$-galactosidase expressing [Neurod6-lineage] cells are present in the thalamus, but could not be assigned to distinct nuclei").

${ }^{4}$ Bashaw and Klein 2010; Vitriol and Zheng 2012; Chen et al. 2012
} 


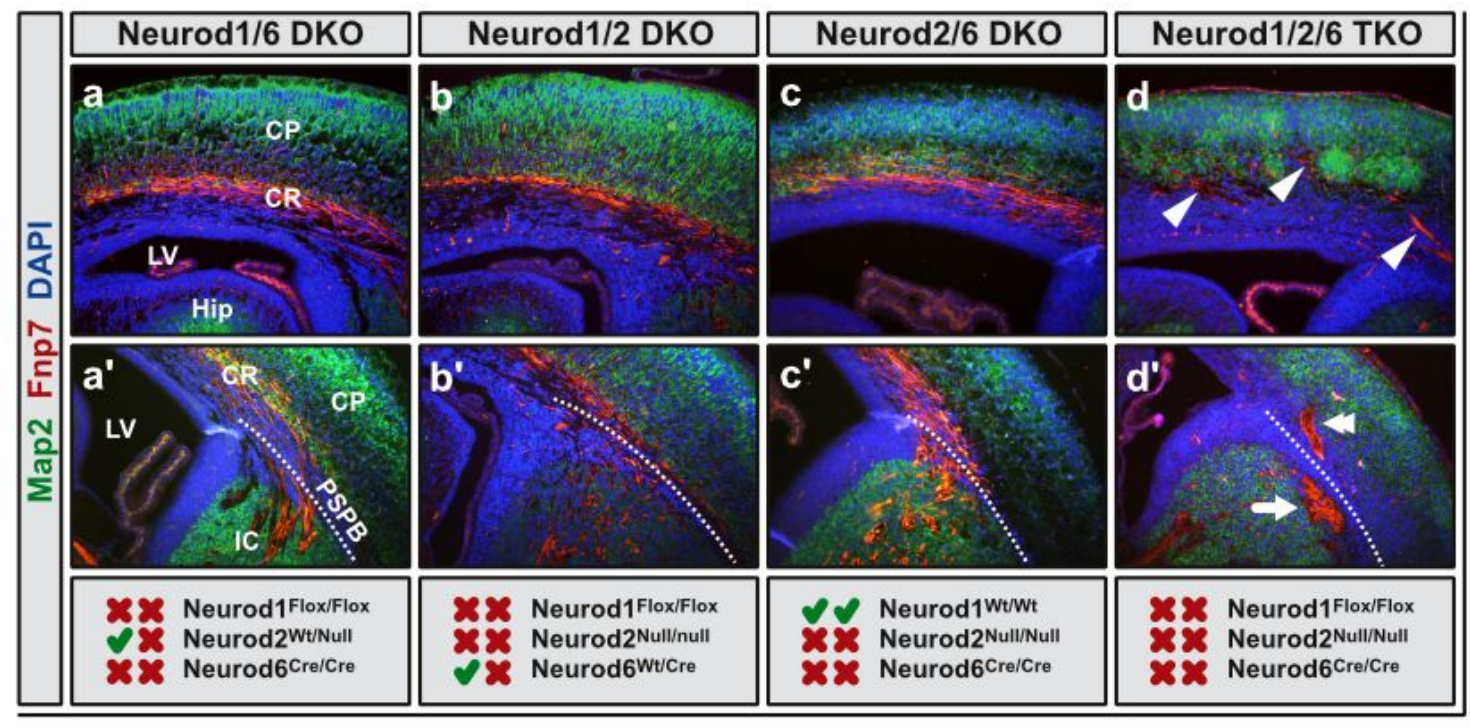

Figure 43: Thalamocortical connectivity in the absence of Neurod1/2/6

IF in coronal paraffin sections of newborn mice. Fnp7 (red) is an antibody recognizing axonally localized non-phosphorylated neurofilaments of medium size. Map2 (green) stains the dendritic compartment of mature neurons and marks the boundary between undifferentiated (VZ, SVZ) and terminally differentiated neuronal tissues (CP, striatum). DAPI nuclear counterstain (blue) is especially enriched in cell-dense proliferative zones that define the PSPB. (a-d) Fnp7-positive projections were visible in the CR (SVZ/CP boundary) of all mice that expressed at least one of the three NeuroD genes, but virtually absent from the triple-deficient neocortex. (a'-d') The proximal TCT could always be identified in the IC. Ascending axons had fasciculated and followed a typical trajectory through the striatum. In mice expressing at least one of the NeuroD genes, thalamocortical axons had crossed the PSPB and had been attracted dorsally into the central neocortex. In Neurod1/2/6 triple-deficient animals, however, they had formed an aberrant, ventrally oriented fiber bundle (arrow). This bundle apparently re-appeared in the pallium (double arrow) without crossing the PSPB at the normal position. The number of Fnp7-positive axons reaching the central neocortex was extremely small (arrow heads).

regular trajectories into the Neurod $1 / 2 / 6$ triple-deficient neocortex (arrow heads in fig $42 \mathrm{~b}$ and in fig $43 \mathrm{~d}$ ').

The inability of most thalamocortical axons to cross the PSPB in Neurod 1/2/6 triple-deficient mice is compatible with the hypothesis that ascending thalamocortical axons depend on physically interaction with descending corticofugal axons to enter the dorsal telencephalon (thalamocortical handshake). ${ }^{1}$

The small number of thalamocortical axons that manage to grow into the Neurod 1/2/6 triple-deficient neocortex may simply correspond to the equally small number of corticofugal axons leaving the triple-deficient neocortex in opposite direction (sect 2.5.6.2). Such quantitative dependency within the handshake model implies that thalamocortical afferents can only be directly guided by corticofugal efferents. Otherwise, if the few thalamocortical axons that had already reached the neocortex were able to guide other thalamocortical afferents in tow, the entire TCT would eventually be able to invade the

\footnotetext{
${ }^{1}$ Molnár et al. 2012: The thalamocortical-corticofugal handshake hypothesis is discussed 20 years after its initial formulation; Chen et al. 2012: Selective genetic ablation of neocortical pyramidal neurons results in the inability of thalamocortical afferents to cross the PSPB. This is currently the strongest evidence in favor of a mandatory handshake between thalamocortical and corticofugal axons.
} 
Neurod 1/2/6 triple-deficient neocortex. The exact molecular mechanisms underlying thalamocortical-corticofugal interactions are yet to be identified, ${ }^{1}$ and Neurod 1/2/6 triple-deficient mice might provide a valuable tool.

\subsection{Other Observations}

$\mathrm{T}$ HE EXPRESSION OF NEUROD-FAMILY transcription factors is not confined to the cerebral cortex (sect 1.4). In collaboration with the laboratory of Carmen Birchmeier (Max Delbrück Center, Berlin) we found Neurod1/2/6 to regulate the differentiation of dynorphin and galanin expressing interneuron subsets of the developing spinal cord in a dose dependent manner. ${ }^{2}$ In collaboration with the group of Connie Cepko (Harvard Medical School, Massachusetts) we showed that Neurod 1/2/6 are expressed in the developing retina and that forced expression of Neurod1, Neurod2 or Neurod6 induces retinal neurogenesis and triggers targeted axon growth in specific sublaminae of the inner plexiform layer. ${ }^{3}$

\footnotetext{
${ }^{1}$ Dwyer et al. 2011: Forward genetic screen based on transgenic mice that express axonally targeted $\beta$-galactosidase in thalamocortical projection neurons. In 'baffled' mutants, most thalamocortical afferents failed to cross the PSPB while a very small number of thalamocortical axons followed normal trajectories into the central neocortex (which resembles the thalamocortical phenotype of Neurod1/2/6 triple-deficient mice). The neocortex was not severely affected in 'baffled' mutants and corticofugal axons crossed the PSPB in close proximity of stalled thalamocortical axons. The underlying mutations have not been identified yet but were speculated to affect the interaction between thalamocortical and corticofugal axons.

${ }^{2}$ Bröhl et al. 2008

${ }^{3}$ Cherry et al. 2011
} 


\section{Discussion}

The mind is an evolved computer
program. The mind program, like the
body, is the result of the evolved DNA
program.

Eric B. Baum ${ }^{1}$

$\mathrm{N}$

EUROD1, NEUROD2 AND NEUROD6 share overlapping expression domains and redundant functions in the cerebral cortex. Every one gene alone is rather dispensable for most aspects of embryonic pyramidal neuron differentiation. But jointly, they control cellular survival, radial migration, subtype identity, and axon guidance in distinct subsets of cells:

Survival: Immature pyramidal neurons that are generated in the caudomedial neocortex or hippocampus depend on Neurod 1/2/6 to promote differentiation and to prevent apoptosis. Note: this does not apply to the rostrolateral cortex.

Migration: Subsets of pyramidal neurons that spread across the neocortex depend on Neurod2/6 to control radial migration. In double-deficient embryos, these cells either fail to initiate or fail to terminate radial migration. Note: most pyramidal cells can migrate independently of Neurod2/6.

Identity: The specification of diverse pyramidal neuron subtypes and molecular identities depends on Neuro $1 / 2 / 6$. As a result, laminarization and arealization of the neocortex are almost lost in triple-deficient mice. Note: corticospinal motoneurons can be specified in absence of Neurod 1/2/6.

Guidance: Targeted axon growth of callosal pyramidal neurons depends on Neurod2/6; in double-deficient mice, callosal axons reach the ipsilateral cingulum, but fail to target the midline. Neurod2 has unique functions in AC formation that cannot be provided by Neurod $1 / 6$. Note: cortical motoneurons can correctly target the spinal cord in Neurod 1/2/6 triple-deficient mice.

The purpose of this work and of still ongoing studies is to identify the roles of Neurod 1/2/6 in cerebral cortex development and to investigate downstream mechanisms of pyramidal neuron differentiation and function.

\footnotetext{
${ }^{1}$ Baum 2004, p 437, chapter 15: What is thought?
} 


\subsection{Determination}

$\mathrm{P}_{\mathrm{d} \text { tes }}$ yRamidal neuron Determination in the cerebral cortex is essentially mediated by Neurogenins (especially Neurog $1 / 2$ ). ${ }^{1}$ Similar to Neurod $1 / 2 / 6$, Neurog $1 / 2 / 3$ form a subfamily of atonal-related bHLH transcription factors (fig 6). In the embryonic cerebral cortex, Neurogenins are preferentially expressed in the $\mathrm{VZ}$ and act as direct counterplayers of the likewise closely related oligodendrocyte differentiation factors Olig $1 / 2 / 3 .^{2}$ Neurogenin expression precedes and triggers the expression of Neurod $1 .^{3}$ Neurog $1 / 2$ are required for the specification of early born but not of later born cortical pyramidal neurons, which constitute deeper and upper layers of the neocortex, respectively. Later neurogenesis depends on an alternative transcriptional program involving paired box gene 6 (Pax6) and Nuclear receptor family $2 \mathrm{e} 1 \mathrm{Nr} 2 \mathrm{e} 1) .{ }^{4}$ In addition to their early expression domain in the cortical VZ, Neurogenins are expressed in a small subset of postmitotic neocortical pyramidal neurons. ${ }^{5}$ This partially resembles the biphasic expression pattern of Neurod1 in the SVZ and CP (sect 1.4.1). Postmitotic Neurog2 expression was shown to influence terminal differentiation ${ }^{6}$ and callosal axon outgrowth ${ }^{7}$ of upper layer pyramidal neurons.

Neurod4, the fourth member of the NeuroD-family of bHLH transcription factors (fig 6), is preferentially expressed by neuronal precursor cells that have left the stem cell state, have started to migrate radially into the SVZ, but do not yet qualify as neurons. ${ }^{8}$ Neurod4 expression is thus believed to define an intermediate stage between undifferentiated neuronal progenitors located in the VZ and basically

${ }^{1}$ Ma et al. 1996, fig 4, 5: Neurog1 overexpression induces ectopic neurogenesis in Xenopus; Nieto et al. 2001, fig 6: Inactivation of Neurog2 and Mash1 results in reduced neurogenesis and premature gliogenesis.

${ }^{2}$ Kageyama et al. 2005, p 347: Neurog2 is able to overwrite the gliogenic effect of Olig1/2; Nieto et al. 2001: Neurog2/Mash1 deficiency leads to reduced neurogenesis and premature gliogenesis in the cortex. Olig genes are essential for oligodendrocyte differentiation, but are also involved in the determination and differentiation of certain types of neurons (Rowitch et al. 2002).

${ }^{3}$ Mattar et al. 2008, fig 2: Double in situ hybridization for Neurog2 and Neurod 1/2/4/6 at 12.5 and E13.5. Talikka et al. 2002, fig 5: Neurog2 directly induces Neurod1 expression (Xenopus).

${ }^{4}$ Schuurmans et al. 2004: At E13.5, the CP of Neurog1/2 deficient mice is largely devoid of the pyramidal neuron markers Neurod6 and Vglut1/2, but harbors a large number of Gad1positive interneurons (fig 1). At E18.5, Neurod6 is robustly expressed in the Neurod1/2 deficient $\mathrm{CP}$ and the relative number of Gad1-positive cells is relatively small (fig 3). The simultaneous inactivation of Pax6 and Nr2el (Tlx) results in an inverse effect (fig 6,7). The authors conclude that Neurog $1 / 2$ and Pax6/Nr2e1 provide two independent mechanisms to specify glutamatergic projection neurons and to inhibit GABAergic fate during early and late cerebral cortex development, respectively (fig 8).

${ }^{5}$ Hand et al. 2005, fig 1: Double immunostaining for Neurog2 and Tuj1 or NeuN demonstrates Neurog2 expression in postmitotic neurons.

${ }^{6}$ Schuurmans et al. 2004, fig 2: Inactivation of Neurog $1 / 2$ results in reduced expression of Neurod6 and Vglut1.

${ }^{7}$ Hand and Polleux 2011, fig 1,3: Inactivation of Neurog2 disturbs callosal axon growth and formation of the corpus callosum.

${ }^{8}$ Roztocil et al. 1997, fig 4 (spinal cord), fig 5 (optic tectum), fig 6 (retina): Neurod4 expression in chicken; Pleasure et al. 2000, fig 1: mouse dentate gyrus at E16.5; Magdaleno et al. 2006: mouse cerebral cortex at E15.5 (image at http://gensat.org/bgem_image_view.jsp?image_id= 12719); Gummert 2003, fig 3I: mouse cerebral cortex at E14.5; Mattar et al. 2008, fig 1B, Q: mouse cerebral cortex at E13.5; Asprer et al. 2011, fig 6: mouse cerebral cortex at E12.5; Johnson et al. 2015, fig 3: mRNA-sequencing of single human embryonic radial glia (neuronal precursor) cells identified the transcriptional profile of Neurod4 to most closely resemble that of Eomes and Neurod 1. 
differentiated migrating progenitors in the SVZ/IZ. Neurog2 and Neurod4 can synergistically activate the Neurod 1 promoter. ${ }^{1}$ In the developing mouse cerebral cortex, Neurod4 expression is relatively weak and most predominant in granule cells of the hippocampal DG. Neurod4 expression in pyramidal cells is confined to the VZ/SVZ boundary, and it is lost upon inactivation of Pax6. ${ }^{2}$ Currently, it is not yet clear whether Neurod 4 is crucial for granule cell differentiation, ${ }^{3}$ and there is no evidence for an essential role in cortical pyramidal neurons. Based on the overlapping expression pattern and high sequence similarity, Neurod4 might promote pyramidal neuron differentiation at the borderline between Neurog $1 / 2$ and Neurod 1 expression.

In the dorsomedial cortex of Neurod $1 / 2 / 6$ triple mutant mice, pyramidal cells fail to differentiate and instead undergo apoptosis shortly after leaving the VZ (sect 2.5.3). In the hippocampal CA, at least one allele of either Neurod1, Neurod2 or Neurod6 is necessary and sufficient to initiate neuronal differentiation and to overcome programmed cell death (sect 2.5.5). Neuronal differentiation in the rostrolateral cortex does not depend on Neurod1/2/6. It is unclear, why hippocampal pyramidal cells are more dependent on early expression of Neurod $1 / 2 / 6$ than pyramidal neurons of the neocortex.

Hypothetically, compensatory upregulation of Neurogenins or Neurod4 in Neurod $1 / 2 / 6$ triple-deficient animals could provide redundant functionality similar to that provided by Neurod 1 in Neurod2/6 double-deficient animals (sect 2.4.7). However, I could not find any indications of significantly increased Neurogenin or Neurod4 expression in the cerebral cortex of Neurod2/6 doubledeficient or Neurod1/2/6 triple-deficient embryos (not shown). It is thus unlikely that Neurogenins or Neurod 4 compensate for the loss of Neurod 1/2/6 outside their natural expression domain. Paradoxically, Neurod4 is predominantly expressed in the caudomedial cortex, ${ }^{4}$ which reacts most sensitive to the loss of Neurod 1/2/6 (sect 2.5.2; fig 34d).

Subsets of pyramidal neurons in the rostrolateral neocortex are able to survive and differentiate largely independent of Neurod $1 / 2 / 6$. In these cells, the identity

${ }^{1}$ Mattar et al. 2008, fig 7: Overexpression of Neurog2 or Neurod4 in P19 cells was sufficient to independently drive luciferase from a $1 \mathrm{~kb}$ Neurod 1 -promoter fragment at comparative levels. An equimolar mixture of Neurog2 and Neurod4 doubled Neurod 1-promoter activity. An equimolar mixture of Neurog2, Neurod 4 and Tcf3 tripled the effect. The synergistic effect of more than two factors suggests the involvement of multiple E-boxes as bHLH proteins generally dimerize to bind DNA (sect 1.3.1).

${ }^{2}$ Visel et al. 2013: Radial migration of pyramidal neurons is disturbed in Pax6 deficient mice. Comparative ISH at E15.5 revealed a complete loss of Neurog $1 / 2$ and Neurod4 expression in the neocortical VZ/SVZ. Neurod 1 expression, which is normally restricted to the SVZ and (at lower levels) to upper layers of the CP, could not be detected in the SVZ but was strongly increased in the CP. Similarly, Neurod6 expression was reduced in the SVZ, but normal in the $\mathrm{CP}$ of the Pax6 deficient embryos.

${ }^{3}$ Pleasure et al. 2000, fig 1, "The Math3 [Neurod4] expression domain in the dentate neuroepithelium is of unclear significance but it may be part of a combinatorial mechanism that, along with Ngn2 and NeuroD, results in the generation of immature granule cells near the ventricular surface.".

${ }^{4}$ Tabata et al. 2013: Comparative expression analysis was performed on a relatively homogeneous subset of cortical neurons. At E15, a GFP expression plasmid was in utero electroporated to the lateral and dorsomedial cortex, respectively. Cortex tissue was triturated after 18 hours and electroporated cells were purified by FACS. Total RNA was extracted and hybridized to Affymetrix 4302.0 GeneChip expression arrays. The ratio of Neurod4 expression in the dorsomedial vs lateral cortex was 2.6 (probe set 1436694_s_at). The expression profile was confirmed by Neurod4 ISH data from the Brain Gene Expression Map (BGEM):

http://gensat.org/bgem_probe_dump.jsp?probe_id=1153 
and functionality of excitatory projection neurons must be instructed by other transcription factors. Pax6 and Nr2e1 or downstream factors such as Sox4/11 might promote pyramidal neuron differentiation in the absence of Neurod $1 / 2 / 6$ (fig 44).

\subsection{Differentiation}

$\mathrm{P}_{\mathrm{lel}}$

YRAMIDAL NEURON DIFFERENTIATION AND RADIAL MIGRATION happen in paralel and influence each other. Both processes are tightly regulated by cell-intrinsic and cell-extrinsic signals. ${ }^{1}$ Even relatively small defects during cortex development can result in abnormal cortical connectivity and severe neurodevelopmental disorders. ${ }^{2}$ Cortex development has been extensively studied for decades, but the molecular control of pyramidal neuron differentiation and of radial migration is far from being completely understood.

In Neurod2/6 double-deficient and Neurod 1/2/6 triple-deficient mice, radial migration is unequally affected in different subpopulations of cortical pyramidal cells: Subsets of immature neurons fail to initiate radial migration and instead accumulate in the $\mathrm{SVZ}$ or $\mathrm{IZ}$ (sect 2.4.2.1; sect 2.4.2.2). Most differentiating pyramidal neurons are able to migrate into the CP. However, some fail to terminate radial migration and consequently invade the $\mathrm{MZ}$. Radial migration and the positioning of neurons within the $\mathrm{CP}$ are also variably affected. In Neurod2/6 double-deficient mice, most pyramidal neurons settle approximately in the correct cortical layer. In Neurod 1/2/6 triple-deficient mice, the laminar organization of the CP is almost lost. Defined groups of similar pyramidal neurons instead segregated into abnormal focal aggregations (sect 2.5.4).

The following sections, summarize the most important stages of normal pyramidal neuron differentiation and radial migration. For every stage, I discuss relevant abnormalities in newborn Neurod2/6 double-deficient and Neurod $1 / 2 / 6$ tripletriple deficient mice. The aim is to speculate on stage-specific functions of Neurod 1/2/6 and to develop testable hypotheses to direct further investigations.

\subsubsection{Ventricular Zone}

Radial glia cells are the neuronal stem cells in the cortical VZ (sect 1.2.3). They are mitotically very active and undergo symmetric divisions to expand the stem cell pool and asymmetric divisions to generate neuronal precursors. Cell cycle activity is paralleled by periodic radial migration events - inter-kinetic nuclear movements. Following symmetric divisions, G1 phase cells move basally, away from the ventricle into the outer VZ. Before the next mitosis, G2 phase cells move apically, back to the ventricular surface. ${ }^{3}$

\footnotetext{
${ }^{1}$ Kandel et al. 2013, chapter 53, p 1051;Cooper 2013; Noctor et al. 2004

${ }^{2}$ Guerrini and Parrini 2010 table 1: List of cortical malformations and associated genes and chromosomal loci.

${ }^{3}$ Cooper 2013, box 1 and sect "Signals that regulate process stability" (p 726ff)
} 
Cell cycle regulation and inter-kinetic nuclear movement in the VZ are most probably independent of Neurod1/2/6. Expression of Neurod1, the earliest of the three genes, starts only as pyramidal neuron precursors transition from the VZ into the SVZ (fig 8; fig 32); the VZ is generally negative for Neurod $1 / 2 / 6$. There were no obvious abnormalities in the $\mathrm{VZ}$ of newborn Neurod 1/2/6 triple-deficient mice (fig 20; fig 35; fig 38).

\subsubsection{Subventricular Zone}

New born pyramidal neuron precursors, which result from asymmetric divisions of radial glia cells, quickly leave the VZ. During early cortex development (E12.513.5 in mice), they immediately lose their mitotic potential, anchor a trailing process in the $\mathrm{VZ}$, migrate into the $\mathrm{IZ}$, and ultimately differentiate into deeper layer pyramidal neurons. During late cortex development (E13.5-E16.5 in mice), they temporarily enter a phase of symmetric mitotic activity within the SVZ, but later also loose the mitotic potential, migrate into the IZ (without leaving a trailing process), and ultimately differentiate into upper layer pyramidal neurons. The secondary mitotic phase in the SVZ lasts for approximately one day (in mice) and expands the total number of upper layer neurons in the cortex. ${ }^{1}$ These intermediate progenitor cells are particularly important in primates and humans where the ratio of upper to deeper layer neurons is much higher than in rodents. During evolution, the increase of secondary neurogenesis in the SVZ resulted in increased intracortical connectivity that probably enabled the emergence of higher brain functions. ${ }^{2}$

The cellular density of the SVZ is increased in newborn Neurod2/6 doubledeficient (fig 20) and Neurod1/2/6 triple-deficient mice (fig 37). Abnormally accumulated cells qualify as undifferentiated pyramidal neuron precursors that robustly express Tbr 1 (fig 20c; fig 38a), are weakly positive for NeuN (fig 20b; fig 37b), but negative for markers that are typically exclusively expressed by terminally differentiated pyramidal neurons, such as Foxp2 (fig 23b; fig 39b), Lmo4 (fig 22b; fig 39b), Map2 (fig 20; fig 37; fig 35; fig 36, fig 43). Neurod6 promoter activity is moderately increased (fig 20d, fig 37c; fig 35b, c; fig 36d, e) and Neurd 1 is expressed in the SVZ of newborn Neurod2/6 double-deficient and Neurod21/2/6 triple-deficient animals (fig 32).

The overall mitotic activity in the neocortical SVZ of Neurod2/6 double-deficient and Neurod $1 / 2 / 6$ triple deficient mice is not obviously increased at birth (Pcna in fig $35 \mathrm{~d}-\mathrm{f}$ ). However, our recent data suggests that the number of Eomes (Tbr2) expressing apical precursor cells is increased in Neurod2/6 double-deficient embryos, and that a larger subset of Eomes/Neurod6-Cre double-positive cells are mitotically active (compare sect 2.1 ).

\footnotetext{
${ }^{1}$ Noctor et al. 2004: Extensive time laps study of clonal cells in the rat cerebral cortex.

${ }^{2}$ Molnár and Pollen 2014
} 


\subsubsection{Intermediate Zone}

After leaving the cell cycle, apical and basal pyramidal neuron precursors migrate further radially into the IZ where they initiate basic neuronal differentiation (sect 1.2.3) and axo-dendritic polarization (sect 1.2.5). At this stage, most neurons are weakly positive for the early neuronal markers NeuN, Tbr1, Ctip2, Satb2 and Neurod6 (Cre), but negative for Foxp2, Cux1, Map2, Mapt and Gap43 (sect 1.2.4; sect 2.1; sect 2.4.2; sect 2.4.4; sect 2.5.4). These basally differentiated (but functionally immature) neurons are termed multipolar because they typically feature several dynamic neurites that do not yet quality as axons or dendrites (sect 1.2.3). They undergo multipolar-to-bipolar transition within the IZ (sect 1.2.5) and initiate axon outgrowth before re-initiating radial migration into the $\mathrm{CP} .1$

Similar to the SVZ (sect 3.2.2), the cellular density of the IZ was increased in newborn Neurod2/6 double-deficient (fig 20) and Neurod1/2/6 triple-deficient mice (fig 37). In Neurod2/6 double-deficient animals, cell accumulations in SVZ and IZ could clearly be separated (fig 20c) and expression levels of NeuN, Neurod6 (Cre) and Ctip2 were higher in the IZ (fig 20b, d; fig 38a) when compared to the SVZ. Neurod $1 / 2 / 6$ triple-deficient mice were generally stronger affected and the borders between SVZ and IZ, or between IZ and CP were only vaguely defines (fig 37b; fig 39a, b; fig 38a, b).

Taken together with sect 1.2.3, two subsets of neuronal cells located in the SVZ and $I Z$, respectively, do critically depend on Neurod2/6 to initiate radial migration into the $\mathrm{CP}$ and to undergo terminal pyramidal neuron differentiation. Systematic studies at earlier embryonic stages are necessary to identify the involved molecular mechanisms and to understand why even in the absence of Neurod 1/2/6 many neocortical pyramidal cells can initiate neuronal differentiation and complete radial migration to the CP. Interestingly, those cells that had accumulated in the SVZ and IZ of newborn Neurod2/6 double-deficient mice disappeared during postnatal brain development, as there were no signs of periventricular heterotopia in two months old Neurod2/6 double-deficient animals (fig 24).

\subsubsection{Cortical Plate}

Polarized neurons in the IZ do immediately initiate tangentially oriented axon growth towards their predefined targets. They also extend prominent, radially oriented dendrites (leading processes) to the $\mathrm{MZ}$ and re-initiate radial migration of the cell bodies to follow the leading processes into the CP (sect 1.2.3). ${ }^{2}$ Radial migration persists inside the $\mathrm{CP}$ and terminates just before the cell bodies reach the MZ. This results in the typical inside-first outside-last layering of the cortex, and it ensures that the MZ stays devoid of pyramidal neurons. Axon growth and neuronal differentiation continue while pyramidal neurons migrate radially and settle in the CP. In fact, ongoing neuronal differentiation and the establishment of particular pyramidal neuron subtype identities are direct prerequisites for the dynamic regulation of targeted axon growth or complex dendritic arborization.

\footnotetext{
${ }^{1}$ Sakakibara and Hatanaka 2015, fig 1B

${ }^{2}$ Noctor et al. 2004; Hatanaka and Yamauchi 2013; Cooper 2014, fig 1;
} 


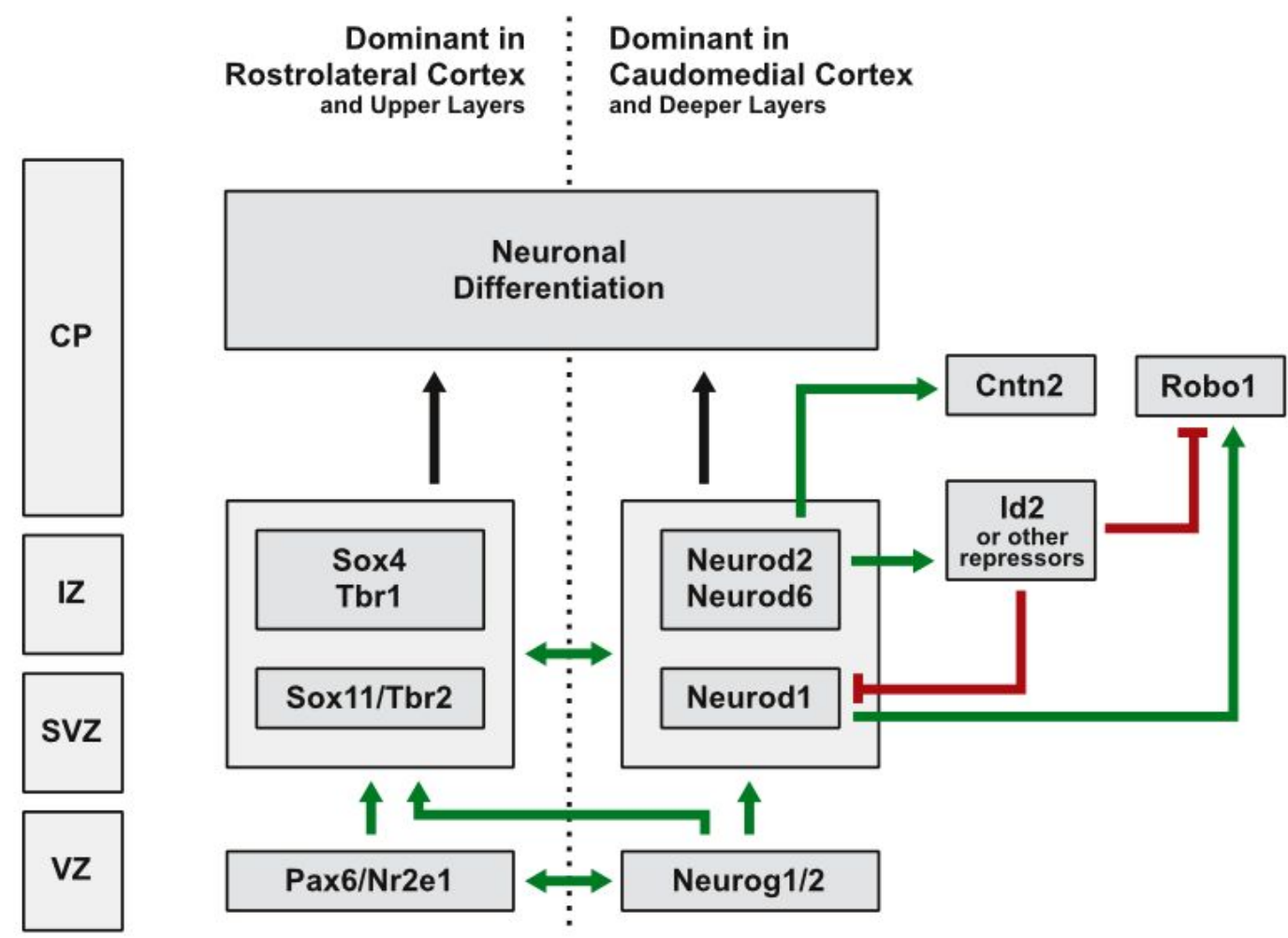

Figure 44: Speculative model of genetic control in the developing neocortex

Example of two interweaved transcriptional modules promote neuronal differentiation in the neocortex. Both modules are principally active in the entire neocortex, but one can dominate the other in particular neurons, stages or regions. The rostrolaterally dominating module is initiated by Pax6/Nr2e1 expression in the VZ and involves e.g. Tbr2/Sox11 and Tbr1/Sox4 which are predominantly expressed in the SVZ and IZ/CP, respectively (Ninkovic et al. 2013; Sansom et al. 2009). Pax6 expression follows a rostrolateral to dorsomedial gradient and Pax6 inactivation results in a rostral shift of neocortical identity (sect 1.2.2). The dorsomedially dominating module is initiated by Neurog $1 / 2$ expression in the VZ and involves e. g. Neurod1 and Neurod2/6 which are predominantly expressed in the SVZ and IZ/CP, respectively (sect Schuurmans et al. 2004). Inactivation of Neurod1/2/6 does primarily affect the dorsomedial cortex (sect 2.5.5). Cntn2 is a potential target gene of Neurod2/6 (sect 2.4.4.3). Robo1 might be (indirectly) repressed by Neurod2/6 and activated by Neurod1, because its expression is increased in the CP of Neurod2/6 double-deficient (fig 28) and decreased in Neurod1/2/6 triple deficient mice (fig 41c). Id2 is a potential candidate for a transcriptional repressor that is controlled by Neurod2/6 and might repress Neurod1 and Robo1 expression in the CP (sect 3.2.4).

Different subsets of pyramidal neurons in the CP of Neurod2 single-deficient (sect 1.5.2; sect 2.3.2), Neurod2/6 double-deficient (sect 2.4.2) and Neurod1/2/6 triple-deficient (sect 2.5.4) mice show very different abnormalities of axonal growth, radial migration, and subtype specification. This heterogeneity suggests that NeuroD-family transcription factors do not simply control a generic master program of pyramidal neuron differentiation, but that the functions of Neurod $1 / 2 / 6$ are heavily interwoven with other inductive factors. The complexity of cerebral cortex development makes is very hard to differentiate cause and effect of particular phenotypical observations. A possible strategy for further functional investigations is to systematically modify the cellular expression levels of selected candidate genes by in utero electroporation. 
Neurod 1 expression is normally confined to the SVZ and absent from polarized pyramidal neurons in the embryonic IZ and CP (fig 8). This pattern is lost in Neurod2/6 double-deficient mice, and the Neurod 1 expression domain expands into the CP (sect 2.4.7). The simplest explanation is that Neurod2/6 can directly or indirectly repress Neurod 1 expression. However, direct repression is unlikely, as Neurod 1/2/6 normally act as transcriptional activators. Indirect repression would involve the induction of yet unidentified transcriptional repressors by Neurod2/6. Potential candidates that should be tested by in utero electroporation include Id2, an inhibitory HLH transcription factor (sect 1.3.2) whose expression is normally robust in the embryonic $\mathrm{CP}^{1}$ but decreased in Neurod2/6 doubledeficient mice $^{2}$ (fig 44).

\subsection{Migration}

$\mathrm{T}$ HE MIGRATION OF DIFFERENTIATING PYRAMIDAL NEURONS in the neocortex can be divided into five consecutive stages: (1) Newly generated neurons or intermediate precursor cells migrate radially from the VZ into the SVZ where they settle temporarily. (2) Postmitotic but immature neurons migrate further radially into the IZ and initiate polarization. (3) During a temporary multipolar stage, the neurons are very dynamic and can move tangentially until completing the multipolar-to-bipolar-transition. (4) Bipolar pyramidal neurons attach to apical processes of radial glia cells and migrate (locomote) radially from the IZ into the upper CP. (5) After detaching from radial glia, the newly arrived neurons enter a last phase of relatively small radial and tangential movements and eventually settle at their final position in the $\mathrm{CP}^{3}$

Stage (1) is not affected by the inactivation of Neurod $1 / 2 / 6$ because none of the three genes is normally expressed in these early cells (sect 1.4; sect 2.1 ). All other migration stages (2-4) are disturbed in different pyramidal neuron subsets of Neurod2/6 double-deficient or Neurod 1/2/6 triple-deficient mice (sect 2.4.2.2; sect 2.5.4.1; sect 3.2).

Particularly interesting is the relatively small subset of well differentiated pyramidal neurons that fail to terminate radial migration in the $\mathrm{CP}$ and instead over-migrate and settle in the $\mathrm{MZ}$ (sect 2.4.2.2; fig 24a). The defect in these pyramidal neurons is clearly cell autonomous because the resident cells of the $\mathrm{MZ}$ are always Neurod1/2/6-negative (sect 2.1) and thus cannot be directly affected by the inactivation of theses genes.

The $\mathrm{MZ}$ is dominated by Reelin secreting Cajal-Retzius cells. Reelin, a diffusible glycoprotein that binds lipoprotein receptors (Apoer2 and Vldlr) on the surface of radially migrating pyramidal neurons. Intracellularly, this leads to phosphorylation of disabled homolog 1 (Dab1) and subsequent activation of lissencephaly 1

\footnotetext{
${ }^{1}$ Neurod 1 expression in wild type tissue:

E15 - http://developingmouse.brain-map.org/experiment/show/100058935

E18 - http://developingmouse.brain-map.org/experiment/show/100076267

${ }^{2}$ Our preliminary data shows reduced Neurod 1 expression in the neocortex of Neurod2/6 doubledeficient and Neurod 1/2/6 triple-deficient embryos (not shown).

${ }^{3}$ Nadarajah et al. 2001; Hatanaka and Murakami 2002; Noctor et al. 2004; Cooper 2014
} 
(Lis 1) and phosphatidylinositol 3-kinase (PI3K) signaling cascades. ${ }^{1}$ Inactivation of either Reelin, ${ }^{2} \mathrm{DAB},{ }^{3}$ or Apoer2 and Vldlr ${ }^{4}$ disrupts Reelin signaling and leads to partial inversion of the CP with the SP being located superficially and the latest born neurons forming the deepest layer. Some of our recent data show that expression levels of Aper2, Vldlr and Dab1 are reduced in the CP of Neurod1/2/6 triple-deficient mice. It is currently not clear if this is a transcriptional effect, whether those genes are also downregulated in Neurod2/6 double-deficient animals, and if such downregulation could be more pronounced in abnormally migrating pyramidal neurons.

\subsection{Arealization}

$\mathrm{T}$ HE PRIMARY SOMATOSENSORY CORTEX is organized in functional units that topographically represent the body surface. Mice, which are nocturnal animals, gather most spatial information from whiskers, that are situated on their snouts. The functional and anatomical units of the whisker cortex are called whisker-barrels. Each barrel corresponds to a single whisker and the cortical barrel map holds a topographic representation of the animal's snout. The formation of whisker-barrels depends on the synaptic activity of thalamocortical axons, and it mainly happens during the first postnatal week, when the pups start to explore their immediate vicinity. During this critical period, pyramidal neurons in layer 4 form strong synaptic contacts with afferent thalamocortical axons that respond to deflection of the corresponding whisker. Contacted pyramidal neurons grow dendritic and axonal processes that mainly terminate within the same whisker-barrel. Pyramidal neurons that respond to the same whisker are strongly interconnected and physically move closer together. This leads to the formation of cell-dens aggregates within layer 4 (the barrels). Transplantation experiments showed that the function and cellular identity of the whisker-barrel cortex are controlled both cell-intrinsically ${ }^{5}$ (by genetic programs) and cell-extrinsically ${ }^{6}$ (by thalamic input).

\footnotetext{
${ }^{1}$ Bock and Herz 2003: Reelin-mediated activation of Src-family non-receptor tyrosine kinases requires ApoE receptors and Dabl activation

${ }^{2}$ D'Arcangelo et al. 1995: Identification Reelin as the gene inactivated in the natural reeler mutant mice

${ }^{3}$ Howell et al. 1997: Genetic inactivation of Dabl in mice leads to a reeler like phenotype

${ }^{4}$ Trommsdorff et al. 1999: Combined inactivation of the VLDL and ApoE2 receptors mimic the reeler phenotype

${ }^{5}$ Cohen-Tannoudji et al. 1994: Visual cortex tissue was isolated from E17/E18 rats and grafted to the somatosensory cortex area of newborn pups. The grafts integrated into the somatosensory barrel field and formed AChE-positive, barrel like cellular aggregates.

${ }^{6}$ Schlaggar and O’Leary 1991: Transgenic mice that carry a H2-K promoter fragment linked to the lacZ gene were accidentally found to express $\beta$-galactosidase specifically in layer 4 pyramidal neurons of the somatosensory cortex. The transgene expression was considered as intrinsic marker of a somatosensory cortex identity. Parietal (somatosensory) and occipital (visual) cortex tissue was isolated from E14/E15 transgenic mice and grafted into the parietal (somatosensory) cortex of newborn, or the cerebellum of P 6 wild type mice. Grafts from the parietal cortex to the cerebellum expressed the transgene, thus maintained the somatosensory identity even in a non-somatosensory environment. Grafts from the occipital to the parietal cortex did mostly not activate the transgene, thus did not quire the somatosensory identity under the influence of the natural somatosensory environment.
} 
The postnatal formation of whisker barrels in the Neurod2 deficient somatosensory cortex is disturbed due to imbalances in NMDA and AMPA mediated neurotransmission (sect 1.5.2). However, the primary arealization of the neocortex is not significantly changed and thalamocortical axons can correctly invade the Neurod2 deficient somatosensory cortex area. ${ }^{1}$ A similar phenotype was reported for Bhlhe22 deficient mice in which the somatosensory cortex area is correctly defined, but the postnatal formation of whisker-barrels is severely disturbed. ${ }^{2}$

In Neurod2/6 double-deficient mice, neocortical arealization is also not completely lost, but the putative somatosensory area is reduced in size and relocated to the caudal neocortex (sect 2.4.6). This resembles the caudal shift of neocortical arealization in Nr2f1 deficient mice (sect 1.2.2). ${ }^{3}$

Neurod 1/2/6 triple-deficient mice are much stronger affected. Arealization of the neocortex is mostly lost and most pyramidal neurons acquire a motoneuron-like identity (sect 2.5.4.3).

A simple hypothesis to explain the increasing defects in Neurod2 single-deficient, Neurod2/6 double-deficient and Neurod 1/2/6 triple-deficient mice is that the functions of the three NeuroD factors are highly redundant, and that the different phenotypes simply result from their incompletely overlapping expression pattern. The postnatal formation of whisker barrels might be disturbed in Neurod2 deficient mice simply because the somatosensory cortex area does robustly expresses Neurod2 but neither Neurod6 nor Neurod1 (fig 9h, d; fig 8d). Prenatally, Neurod2 and Neurod6 are expressed in the same cells and the loss of only Neurod2 can be directly compensated by Neurod6. It is only after birth and in the somatosensory cortex, that the downregulation of Neurod6 exposes the loss of Neurod2 function. The additional inactivation of Neuro6 eventually exposes different functions in earlier pyramidal neurons, and results in defective radial migration, loss of subtype specification and abnormal arealization. Neurod 1 , which is normally only expressed in the SVZ but upregulated in cortical neurons of Neurod2/6 double-deficient mice, can still compensate for the loss of Neurod2/6 in many cells. Only the inactivation of all three NeuroD genes removes all cross compensation and exposes the complete loss-of-function phenotype. In principle, this hypothesis is easily testable by electroporation of Neurod6 (Neurod2 as positive control) into the somatosensory cortex of Neurod2/6 double-deficient embryos. However, it remains to be seen whether in utero electroporation, which typically targets only a small subset of cortical neurons, is sufficient to rescue the formation of detectable whisker-barrels.

\footnotetext{
${ }^{1}$ Ince-Dunn et al. 2006: Whisker formation is not entirely list in Neurod2 deficient mice (CO and DAPI in fig 3; HT5 in fig S4) and DiI tracing confirms that thalamocortical axons target the correct cortex area (fig 3b).

2Joshi et al. 2008: The somatosensory cortex area is correctly defined (fig 4, fig 4) but the molecular identity of pyramidal neurons is abnormal (fig 3).

${ }^{3}$ O'Leary et al. 2007a, fig 5: The frontal and motor areas are enlarged; the auditory, somatosensory and visual areas are smaller and relocated to the caudal border of the neocortex. Postnatal whisker barrel form correctly at the location of the shifted somatosensory field.
} 


\subsection{Axon Growth}

$\mathrm{D}$ IFFERENT SUBTYPES OF PYRAMIDAL NEURONS are primarily characterized by the target sites of their axonal projections. It was shown, that the preferred targets of projection neurons are largely predetermined by cell-intrinsic genetic programs: In vitro differentiated pyramidal neurons that have been grafted into the cerebral cortex of wild type mice do integrate into the host tissue and form axonal projections to subcortical targets. The preferential targets of these axons can be predicted from the time span the precursor cells was allowed to differentiated before grafting. ${ }^{1}$

Callosal, corticothalamic, and corticostriatal projections fail to reach their normal targets in Neurod2/6 double-deficient mice (sect 2.4.4.1, sect 2.4.4.6), while corticospinal axons are able to target the spinal cord even in Neurod1/2/6 triple deficient mice (sect 2.5.6.2). The diversity of molecular identities is lost in triple-deficient animals, and most pyramidal neurons in the CP expressed Ctip2, a typical marker of subcortical and corticospinal projection neurons (sect 2.5.4.1). A simple explanation for these huge differences could be that corticospinal motoneurons are simply the evolutionary oldest type of pyramidal neurons. They might represent a well conserved standard identity, that does not rely on evolutionary younger differentiation programs.

Most transgenic or spontaneous mutant mice published to exhibit callosal agenesis show impairments at very early or later stages of axonal pathfinding. The latter are usually associated with midline glia defects and result in the formation of PBs. ${ }^{2}$ In Neurod2/6 double-deficient mice, PB formation was never observed (sect 2.4.1) and normal expression patterns of Gfap and Blbp in midline glia components suggest that these structures are not primarily affected (sect 2.4.4.4). Moreover, the telencephalic hemispheres fuse at the midline, the $\mathrm{HC}$ forms normally, and pioneer axons originating from the cingulate cortex cross the callosal midline in Neurod2/6 double-deficient mice, demonstrating that midline crossing by commissural axons is in principle possible (sect 2.4.4.4). Many other transcription factors that are expressed by differentiating pyramidal neurons have been genetically inactivated in mice. Most acallosal mutants in which a significant fraction of pyramidal neurons survive until birth form PBs

\footnotetext{
${ }^{1}$ Gaspard et al. 2009: In vitro differentiated cells grafted into the rostral cortex projected to visual cortex, lateral geniculate nucleus, tectum and pontine nuclei

${ }^{2}$ Demyanenko et al. 1999, fig 1, 4: Adult Llcam deficient mice form myelinated PBs but lack a CC; Shen et al. 2002, fig 1,2: Newborn Gap43 deficient mice form PBs but lack CC and AC; Fazeli et al. 1997, fig 8: Newborn Dcc deficient mice form PBs but lack CC and AC; Serafini et al. 1996, fig 8: Newborn Netrin 1 deficient mice form PBs but lack CC and anterior AC; López-Bendito et al. 2007, fig 9: Robol/2 double-deficient mouse embryos form a CC but subsets of callosal axons fail to cross the midline and instead form PB-like structures at the medial septum; Bagri et al. 2002, fig 9: Slit1/2 double-deficient mouse embryos form a thin $\mathrm{CC}$ but most callosal axons fail to cross the midline and instead form PB at the medial septum; Lindwall et al. 2007, tab 1: Table of genes involved in mammalian telencephalic commissure formation.
} 
(eg Pax6, ${ }^{1}$ Tbr $1,{ }^{2} \mathrm{Nr}_{2 \mathrm{f}}{ }^{3}$ and $\mathrm{Emx} 1^{4}$ ). In Emx1/2 double mutants, CC, AC and $\mathrm{HC}$ are absent or reduced in size. ${ }^{5}$ However, Neurod6 and Cntn2 are expressed in the absence of Emx1 and Emx2 whereas Gap43 is absent. ${ }^{6}$ This suggests that Emx1/2 and Neurod2/6 control different genetic programs that are both necessary for neocortical commissure formation.

The absence of Cntn2 expression from Neurod2/6 double-deficient callosal pyramidal neurons might contribute to the premature defasciculation of initially fasciculated callosal fiber bundles. However, reduced Cntn2 protein levels are certainly not the primary cause of callosal agenesis in absence of Neurod2/6, because Cntn2 deficient mice have been reported to develop a grossly normal CC. ${ }^{7}$

Interesting is the strong upregulation of Robol in the CP of newborn Neurod2/6 double-deficient mice (sect 2.4.4.5). Normally, Robol is relatively specific for callosal axons that have already crossed the midline. To prevent back-crossing to the ipsilateral side, growth cones must instantaneously switch from midline attraction to midline repulsion at the moment they initially cross the midsagittal plane. The molecular details underlying these processes are relatively well studied in Drosophila, ${ }^{8}$ and in the mammalian spinal cord and optic chiasm. ${ }^{9}$ Common scheme is the use Netrin/Dcc signaling for midline attraction and Robo/Slit interaction for midline repulsion. The control of midline crossing in the developing $\mathrm{CC}$ is more complex, but seems to follow the same basic principle. Midline attraction is mediated by Netrin/Dcc. ${ }^{10}$ Midline repulsion is mediated by Slit/Robo. ${ }^{11}$ Interestingly, most callosal axons cross the midline and form a $\mathrm{CC}$ in Robol/2 and Slit1/2 double-deficient mice. ${ }^{12}$ Robol has been implicated in the evolution of speech ${ }^{13}$ and is associated with dyslexia in humans. ${ }^{14}$ The cortex areas serving speech processing and production are highly lateralized and thus particularly dependent on callosal connectivity. ${ }^{15}$

In Neurod2/6 double-deficient mice, strong Robol expression by callosal pyramidal neurons and in particular high Robol proteins levels on axons that did not cross the midline (sect 2.4.4.5) might explain the tendency of these axons to

\footnotetext{
${ }^{1}$ Jones et al. 2002, fig 4o: In Pax6 deficient mice, DiI labeled neocortical axons reach the midline and form PBs; only a small fraction cross the midline but fail to enter the contralateral cortex.

${ }^{2}$ Hevner et al. 2001, fig 2C, 7A: In Tbr1 deficient mice, DiI labeled neocortical axons reach the medial cortex and form prominent PBs.

${ }^{3}$ Armentano et al. 2006, fig 4A'-G': In Nr2f1 deficient mice, L1cam stained neocortical axons reach the medial cortex and form prominent PBs.

${ }^{4}$ Gulisano et al. 1996; Chan et al. 2001

${ }^{5}$ Bishop et al. 2003

${ }^{6}$ Shinozaki et al. 2002

${ }^{7}$ Fukamauchi et al. 2001, fig 2B: The CC is visible in coronal brain section at P2. The midsagittal dorsoventral diameter, however, is reduced in all shown sections of Cntn2 deficient animals.

${ }^{8}$ Rajagopalan et al. 2000

${ }^{9}$ Williams et al. 2004

${ }^{10}$ Srivatsa et al. 2014

${ }^{11}$ Unni et al. 2012

${ }^{12}$ López-Bendito et al. 2007

${ }^{13}$ Boeckx and Benítez-Burraco 2014

${ }^{14}$ Hannula-Jouppi et al. 2005

${ }^{15}$ Friederici et al. 2007
} 


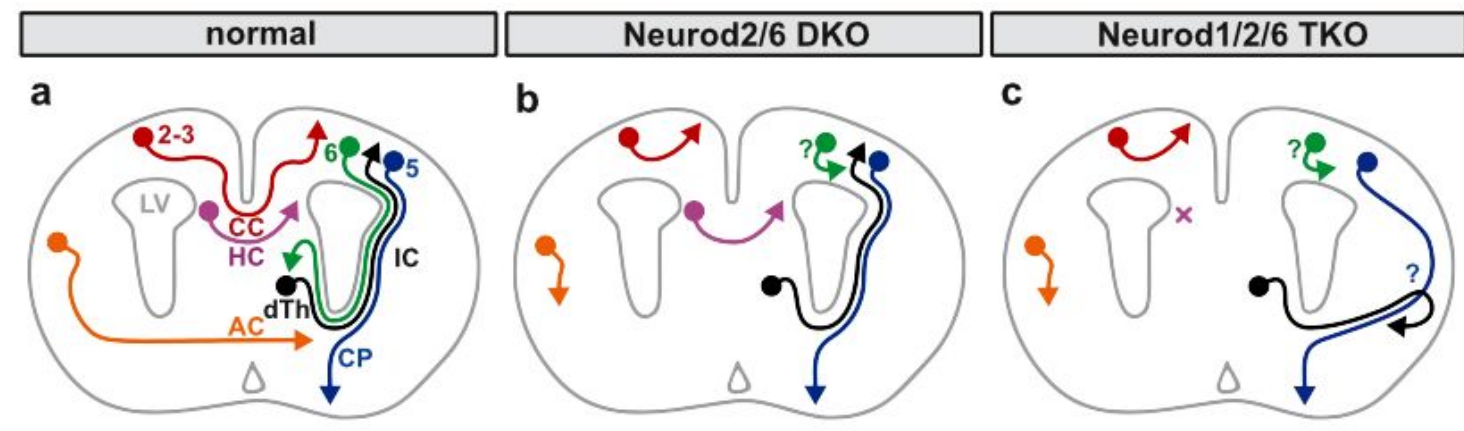

Figure 45: Major cortical fiber tracts in double- and triple-deficient mice

(a) Schematic view of the most important cortical fiber tracts. (b) In Neurod1/6 double-deficient mice, all callosal and subcortical fiber tracts originating from pyramidal neurons of the neocortex were absent. (c) In Neurod1/2/6 triple-deficient animals, the HF did not form due to hippocampal aplasia. Axons of spinally projecting motor neurons grew aberrantly but a subset eventually reached the CST.

invade the $\mathrm{CC}$ of the ipsilateral hemisphere. The cause for this strong Robolupregulation in Neurod2/6 double-deficient mice is unclear as those axons do not contact any midline structures. A simple explanation could be that Robo 1 is normally actively repressed in pyramidal neurons. The responsible repressor might be a target of Neurod2/6 and the loss of repression in Neurod2/6 double-deficient mice may give way to uncontrolled Robol expression.

\subsection{Apoptosis}

$\mathrm{N}$ EURONAL BHLH TRANSCRIPTION FACTORS of the NeuroD-family have previously been shown to control the survival and apoptosis of several neuron populations in vivo, and of cultured neurons and neuron-like cell lines in vitro.

Neurod1 deficiency results in apoptotic loss of hippocampal and cerebellar granule neurons ${ }^{1}$ and reduces adult neurogenesis and neuronal survival in the hippocampus and olfactory bulb. ${ }^{2}$ Neurod2 deficiency leads to increased apoptosis and moderately reduced numbers of cerebellar granule neurons, ${ }^{3}$ non-apoptotic agenesis of the lateral and basolateral amygdala, ${ }^{4}$ and nonapoptotic abnormalities of postnatal neocortex development. ${ }^{5}$ Neurod2 has

\footnotetext{
${ }^{1}$ Miyata et al. 1999; Liu et al. 2000; Schwab et al. 2000; Cho and Tsai 2006

${ }^{2}$ Gao et al. 2009; Kuwabara et al. 2009

${ }^{3}$ Olson et al. 2001, fig 6: TUNEL staining demonstrated increased apoptosis in the cerebellum and hippocampal dentate gyrus of Neurod2 deficient mice; the cerebellar granule cell area was reduced by $\sim 25 \%$.

${ }^{4}$ Lin et al. 2005: Neurod2 deficient mice lack the lateral (LA) and basolateral amygdala (BLA). TUNEL staining at E17/18 (1-2 days after the onset of amygdala formation) did not reveal elevated apoptosis rates. The authors "suggest that formation of the LA and BLA depends on neuroD2 activity".

${ }^{5}$ Ince-Dunn et al. 2006, fig S3: TUNEL staining at P7 showed comparable levels of apoptosis in the cortex of Neurod2-deficient and control mice. The postnatal formation of the somatosensory barrel cortex was disturbed due to reduced thalamocortical synaptic transmission and changes in the ratio of AMPA and NMDA receptor-mediated currents.
} 
recently been identified as a secondary response gene for experimental apoptosis of cerebellar granule neurons in vitro. ${ }^{1}$ Neurod 6 deficiency does not result in known developmental abnormalities. ${ }^{2}$ However, in vitro studies using PC 12 cells implicated Neurod6 in the control of apoptosis. ${ }^{3}$

Pyramidal neurons in the developing cerebral cortex express Neurod1, Neurod2 and Neurod6 (fig 8; fig 9). It was often speculated that NeuroD-family members might be important for pyramidal neuron differentiation and maintenance (sect 1.4). Up to now, such claims could never be experimentally confirmed. In contrast, gene inactivation studies showed that pyramidal neurons can survive and differentiate at least basally in the absence of any one or combinations of two of the three factors (sect 1.5).

As shown here, only the simultaneous inactivation of Neurod1, Neurod2 and Neurod6 results in a significant reduction of cortical gray matter, loss of most pyramidal neurons in the hippocampus and dorsomedial neocortex (sect 2.5.2), and strongly increased apoptotic cell death in these areas (sect 2.5.5). Surprisingly, these effects are area-specific, and the rostrolateral neocortex is relatively unaffected by the inactivation of Neurod $1 / 2 / 6$.

Strictly neuron specific expression pattern of Neurod $1 / 2 / 6$ (sect 1.4 ; sect 2.1) and absence of the proteins from the ventral telencephalon ${ }^{4}$ suggest that most apoptotic cells in Neurod1/2/6 triple-deficient mice are cortical pyramidal neurons. ${ }^{5}$ The nearly complete agenesis of hippocampal CA and dorsomedial CP in Neurod 1/2/6 triple-deficient mice (sect 2.5.2) is probably caused by ongoing neuronal apoptosis during embryonic brain development. TUNEL staining in newborn mice (fig 40) might underestimate the amount of cell death during earlier development because neurogenesis in the neocortex is normally largely complete at birth. Most pyramidal neurons are generated and differentiate during the third trimester of pregnancy.

Further analysis of embryonic cortex development in Neurod 1/2/6 triple-deficient mice is needed to prove the transcriptional control of developmental cell death, to identify underlying molecular mechanisms, and to understand why neuronal survival is differentially regulated in the caudomedial and rostrolateral cortex.

Studies on hippocampal granule cells and inner ear sensory neurons, which likewise die from apoptosis in Neurod 1 deficient mice, suggest that Neurod 1 dependent neuronal survival is promoted by neurotrophin signaling via Ntrk $2 / 3^{6}$ and that apoptosis of Neurod 1 deficient cells is mediated by the activation of

\footnotetext{
${ }^{1}$ Maino et al. 2014, fig 1: Apoptosis was induced in cultured cerebellar granule neurons by lowering the concentration of $\mathrm{KCl}$ in the cell culture medium from 25 to $5 \mathrm{mM}$. The cultures can be protected from the apoptotic effect by the supplementation of gastroinhibitory polypeptide (Gip). Neurod2 expression was decreased in apoptotic cultures and increased in apoptosis protected cultures.

${ }^{2}$ Schwab et al. 1998, 2000; Goebbels et al. 2006

${ }^{3}$ Uittenbogaard and Chiaramello 2005; Uittenbogaard et al. 2009

${ }^{4}$ Schwab et al. 1998; Liu et al. 2000; Olson et al. 2001; Lin et al. 2004; Goebbels et al. 2006

${ }^{5}$ Granule cells of the DG undergo apoptosis in Neurod $1 / 2 / 6$ triple-deficient mice and Neurod $1 / 6$ double-deficient controls (sect 1.5.1). Also not explicitly shown, it is highly unlikely that a significant number of glia cells or interneurons are lost in Neurod 1/2/6 deficient animals.

${ }^{6}$ Kim et al. 2001, fig 6: Expression of the neurotrophin receptors Ntrk2/3 (TrkB/C) was almost absent in the inner ear of Neurod 1 deficient E10.5 mouse embryos. Minichiello and Klein 1996: Simultaneous inactivation of Ntrk2/3 in mice results in apoptotic cell death of hippocampal and cerebellar granule cells and thus resembles the inactivation of Neurod 1.
} 
pro-apoptotic proteins of the Bcl2-family. ${ }^{1}$ It remains to be shown whether these general mechanisms of cell death control are regulated by Neurod $1 / 2 / 6$ in cortical pyramidal neurons. Graded expression pattern of Ntrk2/3 might explain the phenotypical differences in the Neurod $1 / 2 / 6$ triple-deficient rostrolateral and dorsomedial cortex.

\subsection{Genetic Background}

$\mathrm{T}$ HE HERE DESCRIBED DEFICITS of Neurod2-Null mice are more benign than those of the published Neurod2-LacZ animals. ${ }^{2}$ Neurod2-Null homozygotes usually survived adulthood and were hardly distinguishable from littermate controls (sect 2.3.1) while homozygous Neurod2-LacZ mice reportedly developed severe cretinism, failed to thrive, and died around the time of weaning (sect 1.5.2). ${ }^{3}$ Even after thyroxine treatment, which was sufficient to initially overcome adolescent lethality, homozygous Neurod2-LacZ animals had to be sacrificed at the end of the second postnatal month due to progressive neurological decline. ${ }^{4}$

The cause of these phenotypical differences is not known. Remaining Neurod2 gene function, at least, can be ruled out as the entire Neurod2 ORF was removed in both mouse lines. The varying lethality of Neurod2-Null mice crossed into different genetic backgrounds (sect 2.3.1) suggests that other genes modify the role of Neurod2 in development of the hypothalamic-pituitary-thyroid axis. Accordingly, thyroid hormone levels in the blood of postnatal Neurod2-Null mice in the mildly affected $129 \mathrm{X} 1 / \mathrm{SvJ}$ hybrid background were not significantly reduced (preliminary data not shown). Notwithstanding, alterations in thyroid hormone regulation cannot explain the described defects in embryonic brain development, because thyroxine is placenta permeable and provided by the Neurod2 heterozygous mother during gestation.

\subsection{Adult Functions}

N CONTRAST to proneural determination factors, Neurod $1 / 2 / 6$ are expressed by large subsets of terminally differentiated projection neurons of the postnatal cerebral cortex:

\footnotetext{
${ }^{1}$ Kim 2013: Inactivation of Bcl2-associated $X$ protein (Bax) in Neurodl deficient mice does not restore Ntrk2/3 expression but is sufficient to reduce apoptotic cell death in the DG, cerebellum and inner ear.

${ }^{2}$ Olson et al. 2001: Homozygous Neurod2-LacZ mice stopped to gain body weight at P14 (fig 2a) and died between P14 and P35. Even in heterozygous mice, spontaneous seizure activity and unexplained deaths occurred at low incidence.

${ }^{3}$ Olson et al. 2001; Lin et al. 2004, 2005

${ }^{4}$ Lin et al. 2006: Thyroxine treated homozygous Neurod1-LacZ mice survived more than 55 days, while non-treated animals died at P7 to P21, average P14 (fig 4). Treated homozygotes failed to gain appreciable weight and "remained severely neurologically impaired and did not gain appreciable weight despite therapy". "At the 55-day time point, these mice were sacrificed because of neurologic decline".
} 
- Neurod2 is robustly expressed in the adult hippocampus and neocortex. In two months old animals, Neurod 2 mRNA levels are highest in granule neurons of the DG and slightly weaker in pyramidal neurons of the CA and layer $2-6$ of the neocortex. ${ }^{1}$

- Neurod6 mRNA levels are roughly comparable to Neurod2 during adulthood, but restricted to certain subpopulations of cortical projection neurons. In the hippocampus, Neurod6 is absent from most granule cells, but strongly expressed by pyramidal neurons of the entire CA. In the neocortex, Neurod6 is strongly expressed by a subset of pyramidal neurons located predominantly in deeper layers (especially in layer 5), but virtually absent from layer $2 / 3 .^{2}$

- Neurod 1 expression is generally weaker but detectable during adulthood. Neurod 1 mRNA levels are highest in granule neurons of the DG, moderate in pyramidal neurons of the CA (especially CA3), relatively low but detectable in layer $2 / 3$, and virtually absent in layer $4-6$ of the neocortex (sect 1.4.1). ${ }^{3}$ Neocortical Neurod 1 mRNA levels are highest during the first postnatal week and seem to slowly decay during senility. ${ }^{4}$

A recent study provided valuable expression data from laser dissected neocortical tissue $^{5}$ and confirmed that Neurod 1/2/6 (but not Neurog 1/2/3 and Neurod4) are reliably expressed in the postnatal and adult neocortex. Total mRNA levels of Neurod 2 and Neurod6 were comparable and approximately in the range of Tbr 1 and Sox5, but an order of magnitude lower than Mapt or Vglut1. Total mRNA levels of Neurod 1 were an order of magnitude lower than those of Neurod2/6 and approximately in the range of Pax6. At all stages, Neurod 1 was enriched in the supragranular neocortex (upper layers), Neurod6 was enriched in the infragranular neocortex (deeper layers), and Neurod2 showed no consistent laminar enrichment. Longitudinally, total neocortical Neurod 1/2/6 expression levels were highest around birth (at P2), decreased sharply during the first

\footnotetext{
${ }^{1}$ Neurod2 ISH at P56: http://developingmouse.brain-map.org/experiment/siv?id=70437810 ; Lin et al. 2004, fig 3A-G, tab 1: $\beta$-galactosidase histochemistry in brain sections of not further specified adult heterozygous Neurod2-LacZ mice.

${ }^{2}$ Neurod6 ISH at P56: http://developingmouse.brain-map.org/experiment/thumbnails/79544834; Goebbels et al. 2006, fig 4: Neurod6-Cre IHC in the DG of heterozygous Neurod6-Cre mice at P55; Goebbels 2002 fig 18G, H, J, K, fig 19: Neurod6-Cre IHC in the neocortex of three months old heterozygous Neurod6-Cre mice

${ }^{3}$ Neurod1 ISH at P56: http://developingmouse.brain-map.org/experiment/thumbnails/79632311 ; Kim et al. 2002, fig 3: This study showed strong neocortical Neurod 1 expression during the first postnatal week. However, in not further specified adult brains, Neurod 1 mRNA was confined to the DG and CA3, and was not detectable in the neocortex. Absence of Neurod1 ISH signals from the neocortex might be attributed to technical limitations, or alternatively the usage of late adult tissue; Goebbels 2002, fig 18I, fig 20B: In three months old mice, the Neurod 1-LacZ allele is strongly active upper neocortical layers, but detectable in only a small subset of deeper layer neurons; D'Amico et al. 2013: Neurod1 is expressed in postmitotic neurons located in the pallium of adult Xenopus laevis.

${ }^{4}$ Neurod1 ISH at P548: http://developingmouse.brain-map.org/experiment/thumbnails/100082170 Neurodl at P730: http://developingmouse.brain-map.org/experiment/thumbnails/100101386 Neurodl at P1004: http://developingmouse.brain-map.org/experiment/thumbnails/100040743

${ }^{5}$ Fertuzinhos et al. 2014: Neocortical tissue was laser microdissected from the supragranular (pia mater, MZ, layer 2/3), granular (layer 4), and infragranular (layer 5/6, SP, white matter) aspect of the primary somatosensory neocortex at P4, 6, 8, 10, 14 and 180 (one male, one female, each) and total mRNA was used for mRNA and miRNA sequencing (Illumina HiSeq 2000). The primary data (raw sequencing reads) is available from the NCBI Sequence Read Archive at http://ncbi.nlm.nih.gov/sra/?term=SRP031888
} 
postnatal week (especially from P4 to P6), and then remained relatively stable (from P6 to P180).

Robust expression levels of Neurod $1 / 2 / 6$ in subsets of mature projection neurons in the adult cerebral cortex suggest yet unknown roles of NeuroD-family transcription factors in brain function and potentially in psychiatric or neurodegenerative diseases (sect 3.10).

\subsection{Outlook}

Q

UESTIONS for further studies include the following: Which mechanisms do normally prevent developmental cell death of differentiating pyramidal neurons? Which surface molecules qualify callosal axons to cross the cingulate cortex and approach the cortical midline? How is radial migration controlled in different subsets of pyramidal neurons? What are the functions of Neurod $1 / 2 / 4 / 6$ in the adult brain? Do these proteins directly regulate biological processes involved in higher brain functions, developmental cognitive impairments, or neuropsychiatric disorders?

Expression of Neurod $1 / 2 / 6$ is maintained in subsets of mature pyramidal neurons and can still be detected in aged mice. It was speculated that neuronal bHLH proteins might control neuronal plasticity in the adult brain. ${ }^{1}$ Addressing this issue with the mouse mutants used during this study is very difficult because anticipated subtle alterations in adult pyramidal neurons are masked by the above described developmental defects (sect 2.4.3). However, genetic tools have recently been developed that allow to trigger genetic alterations to a well-defined and very restricted spatial and temporal extent.

\subsubsection{Inducible Neurod6-Cre Mice}

Recently, Amit Agarwal (Department of Neurogenetics) generated mice carrying an inducible variant of the Neurod6-Cre allele. In these Neurod6-CreERT2 ${ }^{2}$ animals, fusion of Cre recombinase to a mutated estrogen receptor ligand binding domain results in strictly cytoplasmic localization and inactivity of the protein. Administration of the competitive estrogen receptor antagonist Tamoxifen leads to nuclear translocation of the CreERT2 protein, which is a prerequisite for genetic recombination.

Neurod $1^{\text {Flox } / \text { Flox }} \times$ Neurod 6 Wt/CreERT2 mice allow for the selective inactivation of Neurod 1 in terminally differentiated pyramidal neurons. Neurod $1^{\text {Flox/Flox }} \times$

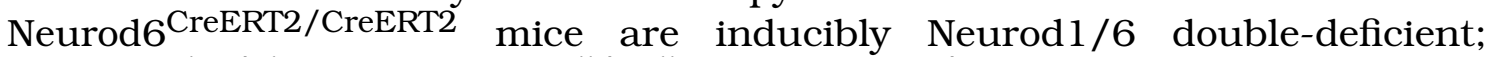
Neurod $1^{\text {Flox/Flox }} \times$ Neurod2 $2^{\text {Null/Null }} \times$ Neurod6 6 Wt/CreERT2 mice are inducibly Neurod $1 / 2$ double deficient. In contrast to conventional Neurod $1 / 2$ or Neurod1/6 double-deficient animals, these are probably not lethal and

\footnotetext{
${ }^{1}$ Soosaar et al. 1994; Schwab et al. 1998; Mikhailova 2008

${ }^{2}$ Agarwal et al. 2012: In Neurod6-CreERT2 (NEX-CreERT2) mice, the Neurod6 open reading frame located in exon 2 was replaced by a Tamoxifen inducible variant of Cre recombinase.
} 
thus suitable to study redundant function in the adult brain. Unfortunately, it is not possible to breed inducible Neurod2/6 double mutant mice with the existing tools. Inducible Neurod $1 / 2 / 6$ triple-deficient mice $\left(\right.$ Neurod $1^{\text {Flox/Flox }} \times$ Neurod2 ${ }^{\text {Null/Null }} \times$ Neurod6 $6^{\text {CreERT2/CreERT2 }}$ ) are conventionally Neurod2/6 double-deficient and certainly perinatally lethal (sect 2.4).

DNA constructs for the conditional targeting of all mouse NeuroD and Neurogenin genes have been generated by the EUCOMM consortium. ${ }^{1}$ Genetically modified ES cells and mice are expected to be available in the near future.

\subsubsection{Neurod6 Overexpression}

To complement the data obtainable by gene inactivation studies, Olga Ucar (Department of Neurogenetics) generated transgenic mice that conditionally express Neurod6 from a strong ubiquitously active promoter. ${ }^{2}$ Crossing these animals to Emx1-Cre mice resulted in a significant decrease of cortical volume, which can be explained by Neurod6-overexpression in mitotic progenitor cells triggering compensated cell cycle exit and neuronal differentiation in the VZ. ${ }^{3}$ Crossbreeding to Neurod6-Cre mice restricted Neurod6-overexpression to the predominantly postmitotic, already neuronally determined Neurod6-Cre lineage of cells (sect 2.1) and did not result in reduced cortical volume. Implications of Neurod6-overexpression on adult brain functions have not been studied because it was not possible to segregate adult gene functions from developmental defects in this mouse model. ${ }^{4}$

Crossbreeding conditionally Neurod6-overexpressing animals to Neurod6CreERT2 mice allows to switch only a fraction of naturally Neurod6-expressing neurons to the overexpressor fate. Administration of a single small Tamoxifen dose during adulthood would result in recombination of only few (until then normally differentiated) Neurod6-overexpressing pyramidal neurons situated within an entirely normally cerebral cortex. Morphology, transcriptional profile or electrophysiological properties of Neurod6-overexpressing pyramidal neurons could be studied in direct comparison to surrounding (genetically normal) control cells.

\footnotetext{
${ }^{1}$ The European Conditional Mouse Mutagenesis Program: http://mousephenotype.org

${ }^{2}$ Mikhailova 2008, sect 2.1 "Production of a mouse line with inducible overexpression of NEX [Neurod6]": The transgenic allele conditionally expresses AU1-tagged Neurod6 under control of the CAG promoter, an artificial sequence consisting of an enhancer element isolated from cytomegalovirus $(C)$, a promoter fragment including the first exon and the transcriptionally active first intron of chicken $\beta$-actin (A), and a splice acceptor of rabbit $\beta$-globin (G): pCAGLoxP-GFP-polyA-LoxP-AU1-Neurod6

${ }^{3}$ Mikhailova 2008, sect 2.2.1 "Overexpression of NEX in cortical progenitors impairs forebrain development": Neurod6-overexpression in the Emx1-Cre lineage resulted in a decrease of cortical surface area (A) by $\sim 20 \%$ (id fig 16C), a reduction of CP thickness (T) by $\sim 10 \%$ (referencing previously cited source (id) fig 17), but no apparent change in neuronal density (D) at birth. The total number of cortical pyramidal neurons $(\mathrm{N})$ can be estimated to be reduced by $25-30 \%(\mathrm{~N}=\mathrm{A} * \mathrm{~T} * \mathrm{D}=80 \% * 90 \% * 100 \%=72 \%)$. This effect is most apparent in layer 6 (id fig 18), suggesting that early born apical progenitor cells in the VZ are more sensitive to Neurod6-overexpression than later born basal progenitors in the SVZ, which have been shown to naturally include a subset of Neurod6 expressing cells that are still mitotically active (sect 2.1, footnote). TUNEL staining and IHC for the neuronally expressed protein Tubb3 at E13.5 confirmed cell death and premature neuronal differentiation in the VZ of Neurod6overexpressing Emx1-Cre mice.

${ }^{4}$ Mikhailova 2008, sect 3.2 and 3.3: The use of CaMKII-Cre mice was proposed instead.
} 
It was shown that developmental mechanisms can be reactivated in the adult brain to switch highly specialized and well integrated neurons to a less mature and more plastic state. ${ }^{1}$ This allows for neuronal plasticity, eg structural changes underlying the modification or extinction of long term memories. Well known plasticity related genes include both, upstream activators ${ }^{2}$ and well as downstream targets ${ }^{3}$ of Neurod6. Adult expression levels of Neurod6 can be modified by the administration of some drugs that are known to alter neuronal plasticity. ${ }^{4}$ Assuming that Neurod6 is a relevant mediator of adult neuronal plasticity, constitutive over-expression should affect structural neuronal plasticity and the modification of long-term memories. Anticipated changes of neuronal morphology are subtle. Experiments must be carefully optimized to reduce side effects affecting brain development or structural integrity. Neurod6-CreET2 mediated modification of adult Neurod6-expression is ideally suited to study such effects, because it does not cause any effects prior to Tamoxifen administration, it does not effect Neurod6-negative cells, and it does not involve invasive procedures such as intracerebral injections.

Recent studies identified effects of adult Neurod 1 overexpression. Retroviral injections into the DG of adult mice increased the functional integration of newly born granule neurons into the hippocampal circuity and were sufficient to rescue morphological and spacial memory deficits in a mouse model of Alzheimer's disease. ${ }^{5}$ Injections into the neocortex of adult mice that had previously been lesioned or that carried multiple Alzheimer's disease mutation resulted in direct conversion of reactive astrocytes into functional glutamatergic neurons. ${ }^{6}$

\subsection{Closing Words}

A S SHOWN, neuronal bHLH transcription factors of the NeuroD-family promote terminal differentiation, survival, migration and targeted axon growth of cortical projection neurons. In particular Neurod2/6 are important for proper development of the neocortex and might modulate higher brain functions or neuropsychiatric diseases. In humans, Neurod6 has recently been associated

\footnotetext{
${ }^{1}$ Ballas et al. 2005: REST and its cofactors (CoREST, MeCP2) are transcriptional repressors that inhibit neuronal differentiation of stem and progenitor cells during development. Inhibition of REST is essential for neuronal differentiation. MeCP2 remains active in terminally differentiated neurons. Synaptic activity and membrane depolarization lead to the release of MeCP2 and thereby disinhibit plasticity related genes such as BDNF. Neurod2 has been shown to transcriptionally inhibit REST (Ravanpay et al. 2010).

Sairanen et al. 2007: Chronic depression often leads to reduced dendritic complexity and hypotrophy in hippocampal and prefrontal cortex areas. These structural changes are reversible and thought to result from stress response and alterations in neuronal plasticity. Chronic treatment with Imipramine, a tricyclic antidepressant, results in increases expression levels of the plasticity related genes PSA-NCAM, pCREB, Gap43 in healthy rats. Gap43 is a known target gene of Neurod6 (sect 2.4.4.3).

${ }^{2}$ Barco and Marie 2011

${ }^{3}$ Grasselli and Strata 2013; Han et al. 2013

${ }^{4}$ Surget et al. 2009

${ }^{5}$ Richetin et al. 2015: Neurog2 or Neurod2 did not cause comparable effects. Overexpression was mediated by stereotaxic injections of retroviruses known to selectively integrate into the genome of dividing cells. Mature neurons were not targeted.

${ }^{6}$ Guo et al. 2014: Retroviral vectors selectively targeting mitotic cells expressed Neurod 1 from GFAP or NG2 promoters, which are active in astroglial or oligodendroglial cells, respectively.
} 
with obsessive-compulsive disorder and Schizophrenia. ${ }^{1}$ Expression levels of Neurod6 and its direct target gene Lppr4 (Prg1) have been identified as possible biomarkers for Alzheimer's disease. ${ }^{2}$ Insufficiency of Tcf4, a heterodimerization partners and modulator of Neurod $1 / 2 / 6$ function in the cerebral cortex ${ }^{3}$ can cause severe neurodevelopmental disorders. ${ }^{4}$ Tcf4 and Tcf4-targeting microRNAs have been repeatedly associated with schizophrenia. ${ }^{5}$

A recent, particularly interesting study compared the genomes of twelve different cattle populations originating from North Africa or the Middle-East. Over the coarse of the last 5000 years, nine of these populations had been introduced to a new sub-Saharan environment and were since under active evolutionary pressure. A scan for the differentiation of single nucleotide polymorphisms (SNPs) in these populations resulted in 42 candidates of highly selected genes. Neurod6 was among the few candidates that are related to the development of function of the nervous system. ${ }^{6}$

To conclude, Neurod1/2/6 regulate a variety of developmental, physiological and pathophysiological processes in the cerebral cortex. Most of the underlying molecular mechanisms are unknown or only in the beginning to be understood.

\footnotetext{
${ }^{1}$ Pérez-Santiago et al. 2012; Mattheisen et al. 2014

${ }^{2}$ Satoh et al. 2014: Comparative analysis of mRNA-sequencing data from two small unrelated postmortem studies of human subjects with Alzheimer's disease ( $n=5+3$, average age $\sim 75$ years) and approximately age matched controls $(n=4+3$, average age $\sim 71$ years) resulted in 522 genes that were differentially regulated in both cohorts (Neurod6 $-89.5 \%, p=0.0023$ and $-84.1 \%$, $\mathrm{p}=0.0006$, respectively; Lppr4 $-71.2 \%, \mathrm{p}=0.0023$ and $-64.4 \%, \mathrm{p}=0.0006)$. The analysis of three unrelated expression array data sets obtained from postmortem brain tissue confirmed the transcriptional downregulation of Neurod6 in Alzheimer's disease.

Yamada et al. 2008: Transient transfection of a Neurod6 expression construct was sufficient to induced Lppr4 expression in primary neuron cultures and PC 12 cells. Chromatin immunoprecipitation in PC12 cells and luciferase assays in HEK cells demonstrated this to be a direct effect of Neurod6 binding to E-boxes located in the proximal Lppr4 promoter.

Carter et al. 2005, fig 2: Expression analysis of inbred mouse models for premature aging revealed Neurod6 to be downregulated in Samp8 inbred mice, which had previously been shown to develop early impairments of learning \& memory, accumulation of A $\beta$ and increased phosphorylation of Mapt, all hallmarks of Alzheimer's disease.

${ }^{3}$ Brzózka et al. 2010 fig 1: Tcf4 interacts with Neurod1 and Neurod2 (co-immunoprecipitation of endogenous proteins in mouse brain lysates). Tcf 4 and Neurod $1 / 2 / 6$ synergistically activate E-box dependent transcription (luciferase assay in primary neuron cultures).

${ }^{4}$ Sepp et al. 2012: Loss of Tcf4 function causes Pitt-Hopkins syndrome, which is characterized by mental retardation, motor deficits and epileptic seizures. Point mutations in Tcf4 can cause the disease if they affect DNA binding or protein-protein interaction (eg, heterodimerization with Neurod2).

${ }^{5}$ Navarrete et al. 2013: "The schizophrenia-associated SNPs [of Tcf4] are in linkage disequilibrium with common variants within putative DNA regulatory elements, suggesting that regulation of expression may underlie association with schizophrenia."

${ }^{6}$ Gautier et al. 2009: Other nervous system related candidate genes include Olfm2, Magil, Sema4a and Htr4. Most other candidates were related to the immune system, skin and hair, which was expected as the animals had been exposed to new parasites and very different climate conditions.
} 


\title{
4 Material and Methods
}

\begin{abstract}
Despite the enormous progress made over the past decade, it is clear that we are still in the early days of defining the logic of neural development, and identifying the players required in different developmental processes.
\end{abstract}

W. Maxwell Cowan ${ }^{1}$

\subsection{Transgenic Mice}

The transgenic mouse lines used for this study are Neurod6-Cre ${ }^{2}$, Neurod2-Null ${ }^{3}$, Neurod1-Flox ${ }^{4}$, and lacZ-based ${ }^{5}$ or GFP-based ${ }^{6}$ Cre-reporters. Animals were housed on wooden fiber in individually ventilated polycarbonate cages that were illuminated in a controlled $12 \mathrm{~h} / 12 \mathrm{~h}$ light/dark cycle. They were fed a standard rodent diet (ssniff Spezialdiäten $\mathrm{GmbH}$ ) and water ad libitum. Experiments were conducted in compliance with the approved animal policies of the Max Planck Institute of Experimental Medicine.

\subsection{Genotyping}

Transgenic mice were genotyped by polymerase chain reaction (PCR) using DNA extracted from small tissue samples. Experimental, specimens were re-genotyped using postmortem tissue.

\footnotetext{
${ }^{1}$ Cowan 1998: Molecular and Cellular Approaches to Neural Development, preface

${ }^{2}$ Goebbels et al. 2006: In Neurod6-Cre (NEX-Cre) mice, the entire Neurod6 open reading frame was replaced by Cre recombinase and a neomycin resistance selection cassette.

${ }^{3}$ Bormuth et al. 2013: In Neurod2-Null mice, the entire Neurod2 open reading frame was replaced by a reversely oriented neomycin resistance cassette.

${ }^{4}$ Goebbels et al. 2005: In Neurod1-Flox (NeuroD-Flox) mice, the entire Neurod1 open reading frame was flanked by LoxP sites.

${ }^{5}$ Soriano 1999: In the Rosa26 Cre-reporter mice, a LoxP-Neo-polyA-LoxP-LacZ-polyA genetrap cassette was inserted into the endogenous Rosa26 locus.

${ }^{6}$ Hippenmeyer et al. 2005: In axonal Cre-reporter (Tau-mGfp) mice, a LoxP-STOP-LoxP-mGFPIRES-nLacZ-polyA targeting cassette was inserted into Exon 2 of the endogenous Mapt locus.
} 
a

\begin{tabular}{l|r|r} 
Substance & Amount & Final Concentration \\
\hline 1 M Tris, pH 8.8 & $67 \mathrm{ml}$ & $67 \mathrm{mM}$ \\
1 M Ammonium sulphate & $16.6 \mathrm{ml}$ & $16.6 \mathrm{mM}$ \\
1 M Magnesium dichloride & $6.5 \mathrm{ml}$ & $6.5 \mathrm{mM}$ \\
Triton X-100 & $5 \mathrm{ml}$ & $0.5 \%$ \\
Water & ad $1000 \mathrm{ml}$
\end{tabular}

\begin{tabular}{l|r|r} 
b $\quad$ Substance & Amount & Final Concentration \\
\hline Tissue lysis buffer (a) & $170 \mu \mathrm{l}$ & \\
Proteinase K, $10 \mathrm{mg} / \mathrm{ml}$ & $20 \mu \mathrm{l}$ & $1 \mathrm{mg} / \mathrm{ml}$ \\
$\beta$-mercaptoethanol & $1 \mu \mathrm{l}$ & $0.5 \%$ \\
Tissue sample & $\max .10 \mathrm{mg}$ &
\end{tabular}

Table 1: Formulation of tissue lysis reaction

(a) Tissue lysis buffer stock solution (b) Tissue lysis reaction for one sample.

\subsubsection{Tissue Lysis}

Small tissue samples were lysed by rigorous shaking at $58^{\circ} \mathrm{C}$ in the presence of Proteinase K, Triton-X and $\beta$-mercaptoethanol (lysis buffer, tab 1). After 3$12 \mathrm{~h}$, the solution was centrifuged using a benchtop centrifuge for $10 \mathrm{~min}$ at $13.000 \mathrm{rpm}$ to remove tissue debris. Without further delay, $5 \mu \mathrm{l}$ of the supernatant were diluted in $200 \mu \mathrm{l}$ of water or $\mathrm{TE}$ and boiled for $20 \mathrm{~min}$ at $95^{\circ} \mathrm{C}$ to inactivate remaining Proteinase $\mathrm{K}$. The resulting DNA-containing solution was directly used in $\mathrm{PCR}$ reactions.

\subsubsection{Polymerase Chain Reaction}

PCR allows for in vitro amplification of DNA sequences defined by a pair of sequence specific primers. ${ }^{1}$ PCR primers are short synthetic oligonucleotides that are designed to be complementary to the ends of the sequence to be amplified. Exponential amplification is achieved by repeatedly exposing a reaction mix containing template DNA, olionucleotide primers, and a thermostable DNA polymerase to different temperatures (tab 2). The denaturation phase at $95^{\circ} \mathrm{C}$ results in ready accessible single stranded DNA. The annealing phase allows the primers to bind complementary template DNA sequences (the annealing temperature depends on the primer sequences and is typically between 50 and $60^{\circ} \mathrm{C}$ ). During the elongation phase at $72^{\circ} \mathrm{C}$ (optimal polymerase activity), bound primers are elongated, thereby synthesizing a second strand that is complementary to the template DNA. Ideally, the absolute number of synthesized copies doubles with every cycle of the PCR program. The most commonly used reaction conditions are given in tab 2.

\footnotetext{
${ }^{1}$ Mullis et al. 1986; Sambrook and Russell 2000, sect 8.1 "The basic polymerase chain reaction"
} 
a

\begin{tabular}{l|r} 
Substance & Amount \\
\hline DNA, ca. $200 \mathrm{ng} / \mu \mathrm{g}$ & $1.0 \mu \mathrm{l}$ \\
Primers, $10 \mathrm{pmol} / \mu \mathrm{g}$ & $0.1 \mu \mathrm{l} \mathrm{each}$ \\
dNTP, $10 \mathrm{mmol}, \mathrm{sum}$ & $0.5 \mu \mathrm{l}$ \\
Standard PCR buffer $(10 \times)$ & $2.0 \mu \mathrm{l}$ \\
Taq polymerase, $1 \mathrm{U} / \mu \mathrm{l}$ & $0.1 \mu \mathrm{l}$ \\
water, PCR grade & $15.7 \mu \mathrm{l}$ \\
\hline$\sum$ & $20 \mu \mathrm{l}$
\end{tabular}

b

\begin{tabular}{ll|r|r} 
Step & Temperature & Time & Loop \\
\hline$\# 1$ & $95^{\circ} \mathrm{C}$ & $180 \mathrm{sec}$ & \\
$\# 2$ & $95^{\circ} \mathrm{C}$ & $30 \mathrm{sec}$ & \\
$\# 3$ & mean primer TM & $30 \mathrm{sec}$ & \\
$\# 4$ & $72{ }^{\circ} \mathrm{C}$ & $35 \mathrm{sec}$ per kbp & $39 \times$ to step \#2 \\
$\# 5$ & $72{ }^{\circ} \mathrm{C}$ & $300 \mathrm{sec}$ & \\
$\# 6$ & $4{ }^{\circ} \mathrm{C}$ & $\infty$ &
\end{tabular}

Table 2: Polymerase chain reaction

Formulation (a) and thermocycler program (b) for standard PCR reactions.

\subsubsection{Gel Electrophoresis}

For genotyping, characteristic genomic DNA fragments were amplified using PCR. Typically, two PCR products of different length were amplified in the same reaction: one to identify the wild type allele, one to identify the modified (transgenic) allele. Gel electrophoresis was used to separate DNA fragments by molecular weight. ${ }^{1}$ Agarose gels contained a DNA binding fluorescent dye to label double strand DNA. Resulting band patterns were visualized using UV light. In the beginning of the experimental work, $0.005 \%$ of the DNA-intercalating fluorescent dye ethidium bromide (EtBr) was used during gel electrophoresis. Concerns over the potential mutagenicity of $\mathrm{EtBr}^{2}$ made us switch to the DNAbinding (not intercalating) cyanine dye SYBR Green.

\subsubsection{Neurod1-Flox PCR}

The forward primer (Neurod1-fw) binds in intron 1 of the Neurod 1 gene. The reverse primer (Neurod1-rv) binds in the Neurod1 ORF. On the wild type allele, this results in a 845 base pair (bp) long amplicon (fig 46a, c). The wild type and transgenic alleles of Neurod1-Flox mice are very similar. Intron 1 only differs by a 166 bp short sequence that contains a LoxP site. ${ }^{3}$ The same primers (Neurod 1-fw

\footnotetext{
${ }^{1}$ Sambrook and Russell 2000, sect 5.2 "Detection of DNA in agarose gels"

${ }^{2}$ Singer et al. 1999: "SYBR Green I stain is a weak mutagen and appears to be much less mutagenic than ethidium bromide"

${ }^{3}$ Goebbels et al. 2005: Intron 1 of the original Neurod1-Flox allele contained a neomycin resistance / thymidine kinase selection cassette that was directly flanked by two LoxP sites. The
} 
a

\begin{tabular}{l|r|r} 
Substance & Amount & Final Concentration \\
\hline Agarose & $5.25 \mathrm{~g}$ & $1.5 \%$ \\
Electrophoresis buffer $(50 \times)$ & $7 \mathrm{ml}$ & $2 \%$ \\
Water & to $350 \mathrm{ml}$ & \\
\hline Ethidium bromide & $17 \mu \mathrm{l}$ & $0.005 \%$
\end{tabular}

b

\begin{tabular}{l|r|r} 
Substance & Amount & Final Concentration \\
\hline Tris-HCl-Base & $242 \mathrm{~g}$ & $2 \mathrm{M}$ \\
Glacial acetic acid & $57.1 \mathrm{ml}$ & \\
$0,5 \mathrm{M}$ EDTA, pH 8 & $100 \mathrm{ml}$ & $1 \mathrm{mM}$ \\
Water & ad $1000 \mathrm{ml}$ &
\end{tabular}

c

\begin{tabular}{l|r|r} 
Substance & Amount & Final Concentration \\
\hline Bromphenol blue & $100 \mu \mathrm{l}$ & $0.1 \%$ \\
Xylencyanol & $100 \mu \mathrm{l}$ & $0.1 \%$ \\
Ficoll (Type 400) & $15 \mathrm{ml}$ & $15 \%$ \\
Water & ad $100 \mathrm{ml}$ &
\end{tabular}

Table 3: DNA gel electrophoresis

(a) Formulation for the preparation of agarose gels. (b) TAE electrophoresis buffer (50x). (c) DNA loading dye (10x).

and Neurod1-rv) amplify $1011 \mathrm{bp}$ of the Neurod1-Flox transgenic allele (fig 46b, c). In heterozygous mice, both amplicons can heterodimerize due to high sequence similarity, which often results in a third band running at approximately $928 \mathrm{bp}$ in gel electrophoresis.

\subsubsection{Neurod2-Null PCR}

The Neurod2-Null ${ }^{1}$ PCR (fig 47) uses a common reverse primer (Neurod2-rv) that binds in the 3'-untranslated region (UTR) of the Neurod2 gene. The forward wild type primer (Neurod2-fw) binds inside the Neurod2 ORF. The resulting $971 \mathrm{bp}$ spanning amplicon is specific for the Neurod2 wild type allele (fig 47a, c). The forward transgene primer (Neo-rv) binds inside the reversely-oriented neomycin resistance cassette. This is a forward primer in relation to the entire Neurod2-Null allele, but it is a reverse primer in relation to the neomycin resistance cassette itself (fig 10; fig 47b). Together with the common reverse primer, a $\sim 680 \mathrm{bp}$ spanning fragment is amplified specifically from the Neurod2-Null transgenic allele (fig $47 \mathrm{~b}, \mathrm{c}$ ). Due to the inaccessibility of the genomic Neurod2 locus, a modified PCR reaction buffer was used for Neurod2 genotyping ${ }^{2}$ (fig 47d, e).

selection cassette was removed by partial Cre mediated recombination in mice which left only one LoxP site in intron 1. Another LoxP site is located in the 3'-UTR.

${ }^{1}$ Bormuth et al. 2013: Targeting and genotyping of Neurod2-Null mice (fig 1).

${ }^{2}$ Kogan et al. 1987 
a

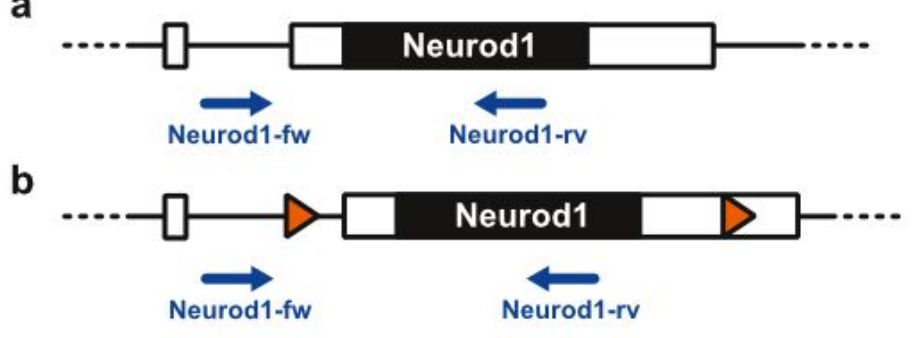

C

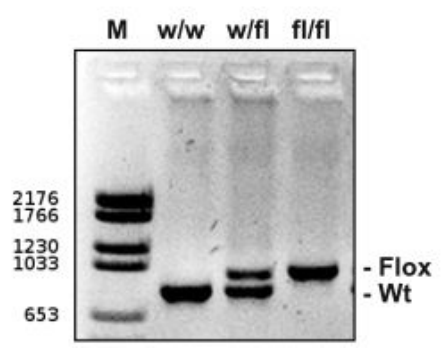

d

\begin{tabular}{l|l} 
Primer & Sequence \\
\hline Neurod1-fw & 5'-GTTTTTGTGAGTTGGGAGTG-3' \\
Neurod1-rv & 5'-TGACAGAGCCCAGATGTA-3'
\end{tabular}

Figure 46: Neurod1-Flox genotyping PCR

(a, b) Schematic overview of primer binding sites in wild typeNeurod1 (a) and modified Neurod1-Flox (b) alleles. (c) Gel electrophoresis after Neurod1-Flox PCR.(d) Primer sequences.

\subsubsection{Neurod6-Cre PCR}

The Neurod6-Cre ${ }^{1}$ PCR (fig 48) uses a common forward primer (Neurod6-fw) binding in the first intron of the Neurod6 gene. The reverse wild type primer (Neurod6-rv) binds inside the Neurod6 ORF located in exon 2. The resulting amplicon is $771 \mathrm{bp}$ long and specific for the Neurod6 wild type allele (fig 48a, c). The reverse Cre primer (Cre-rv) binds in Cre recombinase complementary DNA (cDNA) and generates an amplicon that is $525 \mathrm{bp}$ long and specific for the Neurod6-Cre transgenic allele (fig 48b, c).

\subsection{Cell Culture}

Brains were quickly dissected from newborn mice in ice-cold Hank's balanced salt solution (HBSS) containing $0.15 \% \mathrm{MgSO}_{4}$ (HBSS+). Using a binocular microscope, the neocortex was prepared in ice-cold HBSS+: The cerebral hemispheres were separated from the diencephalon, meninges were removed, the olfactory bulb and the hippocampus were removed by strait coronal cuts, a parasagittal incision was made to remove the cingulate cortex, and a horizontal cut was made to remove the lateral cortex. These four incisions also separated the cortex from the basal ganglia, which could now be removed along the ventricular volume. The neocortex was put into a solution of papain $(10 \mathrm{u} / \mathrm{ml})$ and DNase $(0.25 \mathrm{mg} / \mathrm{ml})$ in HBSS+ and was incubated at $37^{\circ} \mathrm{C}$ for $30 \mathrm{~min}$ (the tube was gently inverted every 5-10 min). Cells were carefully triturated using a Pasteur pipette. The suspension was diluted in ice-cold HBSS+ to a volume of $12 \mathrm{ml}$. The tube was kept on ice for $30 \mathrm{sec}$ to let tissue debris sink to the bottom. The upper $11 \mathrm{ml}$ of the suspension was transferred to a new tube, a $20 \mu \mathrm{l}$ sample was removed for cell-counting, and the remainder was immediately centrifuged at $800 \mathrm{~g}$ for $5 \mathrm{~min}$.

\footnotetext{
${ }^{1}$ Goebbels et al. 2006: Targeting and genotyping of Neurod6-Cre (NEX-Cre) mice (fig 1)
} 
a

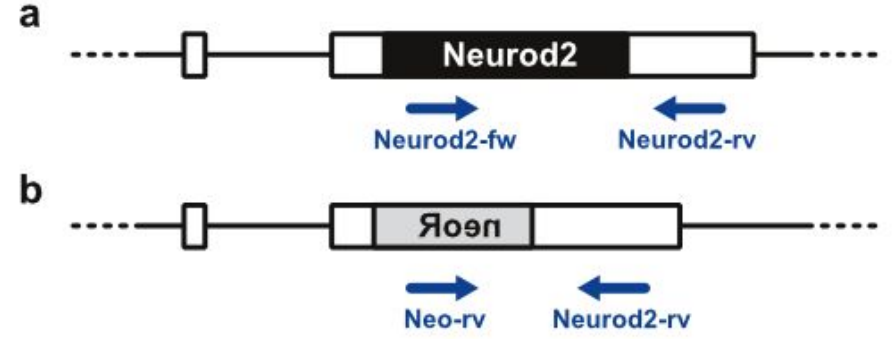

C

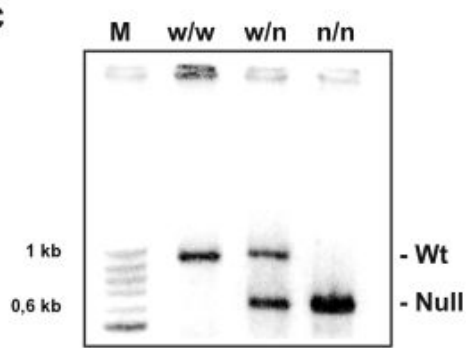

\begin{tabular}{|c|c|c|c|}
\hline \multirow[t]{4}{*}{ d } & Primer & \multicolumn{2}{|l|}{ Sequence } \\
\hline & Neurod2-rv & \multirow{3}{*}{\multicolumn{2}{|c|}{$\begin{array}{l}\text { 5'-CTGTTGGGAGGTGGGGAGAG-3' } \\
\text { 5'-TGGGCTCTCTCGGAGATCT-3' } \\
\text { 5'-ATTGTCTGTTGTGCCCAGTC-3' }\end{array}$}} \\
\hline & Neurod2-fw & & \\
\hline & Neo-rv (fw in allele) & & \\
\hline \multirow[t]{10}{*}{ e } & Substance & Amount & \\
\hline & DNA, 200 ng/ul & $2.0 \mu \mathrm{l}$ & \\
\hline & Primers, $50 \mathrm{pmol} / \mu \mathrm{l}$ & each $0.1 \mu l$ & \\
\hline & dNTP, 10 mmol (sum) & $2.0 \mu \mathrm{l}$ & \\
\hline & DMSO & $2.5 \mu \mathrm{l}$ & \\
\hline & $\mathrm{BSA}, 10 \mathrm{mg} / \mathrm{ml}$ & $0.2 \mu \mathrm{l}$ & \\
\hline & Soriano PCR Buffer & $5.0 \mu \mathrm{l}$ & \\
\hline & Taq Polymerase, $1 \mathrm{U} / \mu \mathrm{l}$ & $0.1 \mu \mathrm{l}$ & \\
\hline & Water, PCR grade & $9.4 \mu \mathrm{l}$ & \\
\hline & $\sum$ & $20 \mu \mathrm{l}$ & \\
\hline & Substance & Amount & Final Concentration \\
\hline & $1 \mathrm{M}$ Ammonium sulphate & $4.15 \mathrm{ml}$ & $83 \mathrm{mM}$ \\
\hline & $1 \mathrm{M}$ Tris, $\mathrm{pH} 8.8$ & $16.75 \mathrm{ml}$ & $335 \mathrm{mM}$ \\
\hline & $1 \mathrm{M}$ Magnesium dichloride & $1.68 \mathrm{ml}$ & $33.5 \mathrm{mM}$ \\
\hline & $\beta$-mercaptoethanol & $1.25 \mathrm{ml}$ & $25 \mathrm{mM}$ \\
\hline & 0,005 M EDTA, pH 8 & $334 \mu \mathrm{l}$ & $33.5 \mathrm{mM}$ \\
\hline & Water & ad $50 \mathrm{ml}$ & \\
\hline
\end{tabular}

Figure 47: Neurod2-Null genotyping PCR

(a, b) Schematic overview of primer binding sites in wild type Neurod2 (a) and modified Neurod2-Null (b) alleles. (c) Gel electrophoresis after Neurod2-Null PCR. (d) Primer sequences. (e, f) Formulation of modified PCR reaction (e) and $5 \times$ PCR buffer (f).

Cells were counted manually using an improved Neubauer hemocytometer. The pellet was resuspended in Neurobasal medium (Invitrogen) supplemented with $100 \mathrm{IU} / \mathrm{ml}$ Penicillin, $0.1 \mathrm{mg} / \mathrm{ml}$ Streptomycin, $100 \%$ Glutamax (Invitrogen) and $2 \%$ B27 (Invitrogen) to a final concentration of 2 million cells per ml. Cells were plated on PLL coated glass coverslips ( $7 \mathrm{~mm}$ diameter) using $30 \mu \mathrm{l}$ cell suspension $\left(60,000\right.$ cells per coverslip). The cultures were incubated at $37^{\circ} \mathrm{C}, 5 \% \mathrm{CO}_{2}$ for up to one month. 
a
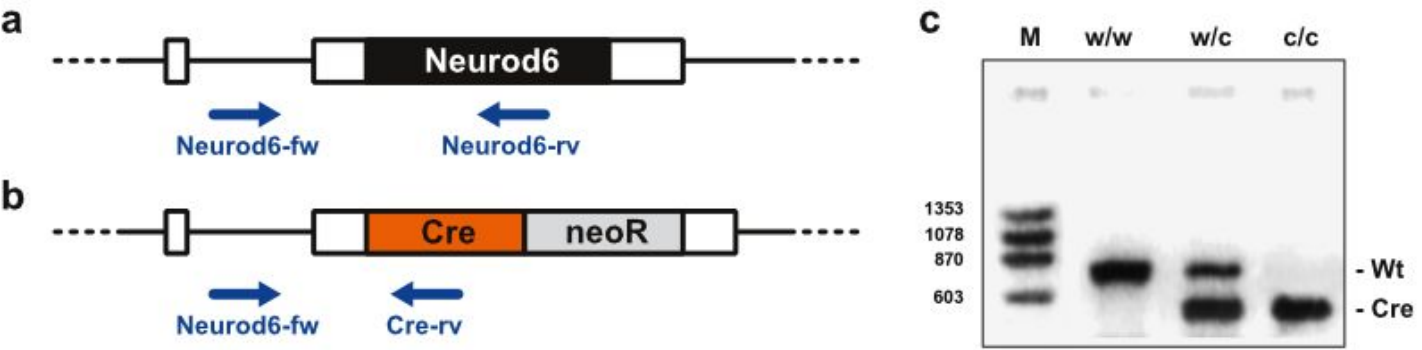

\begin{tabular}{|c|c|}
\hline Primer & Sequence \\
\hline Neurod6-fw & 5'-GAGTCCTGGAATCAGTCTTTTTC-3' \\
\hline Neurod6-rv & 5'-AGAATGTGGAGTAGGGTGAC-3' \\
\hline Cre-rv & 5'-CCGCATAACCAGTGAAACAG-3' \\
\hline
\end{tabular}

Figure 48: Neurod6-Cre genotyping PCR

(a, b) Schematic overview of primer binding sites in wild type Neurod6 (a) and modified Neurod6-Cre (b) alleles. (c) Gel electrophoresis after Neurod6-Cre PCR. (d) Primer sequences.

\subsection{In Utero Electroporation}

Pregnant mice were anesthetized by intraperitoneal injection of $10 \mathrm{~g}$ xylazine and $100 \mu \mathrm{g}$ ketamine per gram body weight. A subcutaneous injection of $0.1 \mu \mathrm{g}$ per gram body weight buprenorphin was given to ensure complete analgesia. The uterine horns were exposed, and plasmid DNA was injected into the embryonic lateral ventricle using a glass pipette connected to an automated micro injector pump. Electroporation (six times at $50 \mathrm{~ms}, 33$ Volts direct current with $100 \mathrm{~ms}$ intervals) was performed using an ECM 830 electroporator (BTX). The wound was surgically sutured, and the mouse was monitored until full recovery from anesthesia. The procedure had been approved by the animal welfare officer of the Max Planck Institute of Experimental Medicine and is covered by Gen 33.42502-04-035/07. The entire procedure has been described in detail in Saito 2006 (original publication) and Walantus et al. 2007 (video protocol).

\subsection{Electrophysiology}

Electrophysiological analysis was performed using dissociated neocortical neuron cultures after 21-28 days in vitro. The bath solution in all experiments comprised $120 \mathrm{mM} \mathrm{NaCl}, 3 \mathrm{mM} \mathrm{KCl}, 2 \mathrm{mM} \mathrm{CaCl}_{2}, 2 \mathrm{mM} \mathrm{MgCl}_{2}, 10 \mathrm{mM} \mathrm{HEPES}$, and $10 \mathrm{mM}$ glucose $(\mathrm{pH} 7.35$, continuously aerated with a mixture of $95 \%$ $\mathrm{O}_{2}$ and $5 \% \mathrm{CO}_{2}$ and kept at $32^{\circ} \mathrm{C}$ ). The pipette solution for all experiments

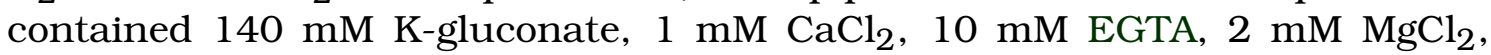
$4 \mathrm{mM} \mathrm{Na} 3 \mathrm{ATP}, 0.5 \mathrm{mM} \mathrm{Na} \mathrm{m}_{3} \mathrm{GTP}$, and $10 \mathrm{mM}$ HEPES (pH 7.3). SEPSCs were recorded in the presence of $1 \mu \mathrm{M}$ bicuculine. P-values represent the results of a two-tailed unpaired Student's t-tests, with or without Welch's correction, depending on the distribution of the data (tested with a Kolmogorov-Smirnov test). Data acquisition and analysis were performed using commercially available 


\begin{tabular}{|c|c|c|}
\hline Gene & Orientation & Sequence \\
\hline$\beta$-actin & $\begin{array}{l}\text { forward } \\
\text { reverse }\end{array}$ & $\begin{array}{l}\text { 5'-CTCTGAACCCTAAGGCCAACC-3' } \\
\text { 5'-GGCATACAGGGACAACACAGC-3' }\end{array}$ \\
\hline Cntn1 & $\begin{array}{l}\text { forward } \\
\text { reverse }\end{array}$ & $\begin{array}{l}\text { 5'-CCTCATTCCGATTCCTGAAC-3' } \\
\text { 5'-TCCTTGAACTGCACCACAAT-3' }\end{array}$ \\
\hline Cntn2 & $\begin{array}{l}\text { forward } \\
\text { reverse }\end{array}$ & $\begin{array}{l}\text { 5'-GAATCCGCACTAAGGAAGCA-3' } \\
\text { 5'-CCTGATTGTACTTCAGCCCTTT-3' }\end{array}$ \\
\hline Gap43 & $\begin{array}{l}\text { forward } \\
\text { reverse }\end{array}$ & $\begin{array}{l}\text { 5'-GGAAGAGAGGAGGAAAGGAGA-3' } \\
\text { 5'-TGGTCCTCATCATTCTTTTCAA-3' }\end{array}$ \\
\hline Neurod1 & $\begin{array}{l}\text { forward } \\
\text { reverse }\end{array}$ & $\begin{array}{l}\text { 5'-CCTTTGACGGACCCCTCAG-3' } \\
\text { 5'-CGGATGGTTCGTGTTTGAAA-3' }\end{array}$ \\
\hline Neurod2 & $\begin{array}{l}\text { forward } \\
\text { reverse }\end{array}$ & $\begin{array}{l}\text { 5'-ССТCTCCCCTTGACTCCTCT-3' } \\
\text { 5'-GAACAGGCGGGTCAGCAT-3' }\end{array}$ \\
\hline Tbr1 & $\begin{array}{l}\text { forward } \\
\text { reverse }\end{array}$ & $\begin{array}{l}\text { 5'-CAAGGGAGCATCAAACAACA-3' } \\
\text { 5'-GTCCTCTGTGCCATCCTCAT-3', }\end{array}$ \\
\hline
\end{tabular}

Table 4: Primer sequences for qRT-PCR

Primer pairs amplify approximately $80 \mathrm{bp}$ of the target cDNA.

software: pClamp 9.0 (Molecular Devices), MiniAnalysis (SynaptoSoft, Decatur, GA) and Prism 4 (GraphPad Software, San Diego, CA). Electrophysiology was performed together with Ming Zhang, Department of Neurophysiology, University of Göttingen (now Laboratory of Molecular Psychiatry, Department of Psychiatry, University of Münster), Germany.

\section{6 qRT-PCR}

Total ribonucleic acid (RNA) was isolated from cortex tissue using RNeasy Micro Columns (Qiagen). Reverse synthesis of cDNA was performed using SuperScript III RNase H Reverse Transcriptase (Invitrogen) with a mix of random nonamer and anchored poly-dT primers. PCR reactions were based on the Fast SYBR Green Mastermix (Invitrogen \#4385610). Thermocycling and parallel data acquisition were performed on a 7500 Fast Real-Time PCR System (Applied Biosystems) using default settings. The qRT-PCR primers were designed using the Universal Probe Library (Roche) primer design tool. ${ }^{1}$ Primer sequences are given in tab 4 . Relative target mRNA concentrations were calculated by normalizing to $\beta$-actin.

\footnotetext{
${ }^{1}$ Universal Probe Library design tool for mouse genes: http://qpcr.probefinder.com/input.jsp?organism=m_mus
} 


\subsection{Histology}

\subsubsection{Tissue Preparation and Fixation}

Postnatal mice were anesthetized with $0.2 \mathrm{ml}$ per $10 \mathrm{~g}$ body weight avertin $(12.5 \%$ 2,2,2-tribromethanol and 2.5\% 2-methyl-2-butanol in $\mathrm{H}_{2} \mathrm{O}$ ) and intracardially perfused with $15 \mathrm{ml}$ of HBSS, followed by $25 \mathrm{ml}$ neutrally buffered formalin (NBF). Brains were removed and postfixed in NBF for $2 \mathrm{~h}$ to overnight at $4^{\circ} \mathrm{C}$. Embryos and newborn pups were quickly killed by cervical dislocation. To prevent preparation artifacts, the skull was carefully opened and whole heads were fixed in $10 \% \mathrm{NBF}$ for $1 \mathrm{~h}$. Fixed brains were removed from the skull and postfixed in $\mathrm{NBF}$ for $2 \mathrm{~h}$ to overnight at $4^{\circ} \mathrm{C}$.

\subsubsection{Tissue Sectioning}

Depending on the experimental requirements, different sectioning techniques were used. Thick vibratome sections were preferred for the visualization of fluorescently labeled axon tracts and for the detection of native proteins by direct green fluorescent protein (GFP) fluorescence or X-Gal histochemistry (sect 4.7.3). Cryosections are best suited for ISH (sect 4.7.6) and for IHC using antibodies that only bind native epitopes (sect 4.7.5; tab 6). Thin paraffin sections were used for standard histological staining, myelin staining and IHC with suitable antibodies, because they are easily processed in large quantities and can be stored at room temperature (RT) for years.

\subsubsection{Vibratome}

If possible, fresh tissue was used for vibratome sectioning. Alternatively fixed specimens were stored in $30 \%$ sucrose in phosphate buffered saline (PBS) containing $0.1 \%$ sodium azide for up to two months at $4{ }^{\circ} \mathrm{C}$. Tissue was cut into 20-200 $\mu \mathrm{m}$ thick sections using a Leica vibratome filled with ice-cold PBS. The blade's vibration speed was set to maximum and the forward driving speed to 5-30 \% (lower for thinner sections). Sections were transferred to PBS and stored for up to two weeks at $4^{\circ} \mathrm{C}$.

\subsubsection{Cryostat}

To inhibit the formation of ice crystals during freezing, fixed tissue sample were incubated overnight in $30 \%$ sucrose in PBS at $4^{\circ} \mathrm{C}$. For freezing, small tubes were made from aluminum foil, labeled with appropriate spatial axes and filled with cryostat embedding medium (Tissue-Tec, OCT). Specimens were transferred into these tubes at RT and were carefully positioned to match the future cutting plane. Fast but gradual freezing was performed by slowly lowering the tubes into liquid nitrogen using long forceps. Frozen samples were stored at $-80^{\circ} \mathrm{C}$.

The tissue was sectioned using a Leica cryostat CM3050. Sample temperature was set to a value between -18 and $-30^{\circ} \mathrm{C}$ (lower temperature for embryonic tissue) and the chamber temperature was set to a value $2^{\circ} \mathrm{C}$ lower than that of 
the sample. Frozen tissue was slowly cut to 14-25 $\mu \mathrm{m}$ thick sections, which were immediately transferred to adhesive glass slides (Histobond, Superfrost).

Glass slides containing freshly cut tissue sections were kept at RT for approximately 30 min and were subsequently dried in a vacuum desiccator containing silica granules for $3-8 \mathrm{~h}$ at RT. Slides containing dried tissue sections were transferred into a plastic box containing silica granules. The box was sealed using duct tape and stored at $-80^{\circ} \mathrm{C}$. Just before use, slides were thawed inside a silica granule-filled vacuum desiccator.

\subsubsection{Paraffin}

Fixed tissue was dehydrated by sequential incubation in deionized water, $50 \%$ ethanol, $70 \%$ ethanol, $90 \%$ ethanol, $95 \%$ ethanol, $100 \%$ ethanol (twice), isopropanol/xylene (1:1), xylene (twice) and liquid paraffin (twice at $60^{\circ} \mathrm{C}$ ), approximately $2 \mathrm{~h}$ each. Brains were embedded in liquid paraffin on a hot plate at $65^{\circ} \mathrm{C}$ and quickly chilled on a cold plate to $-7^{\circ} \mathrm{C}$. To increase reproducibility, multiple control and mutant brains were processed in parallel and embedded into the same paraffin block. Tissue was cut into 5-10 $\mu \mathrm{m}$ thick sections using a sliding microtome (Leica), dried at RT, incubated at $37^{\circ} \mathrm{C}$ overnight and stored at RT for unlimited time.

For further processing, paraffin sections were deparaffinized using xylene and rehydrated by sequential incubation in xylene/isopropanol (1:1), $100 \%$ ethanol, $90 \%$ ethanol, $70 \%$ ethanol, $50 \%$ ethanol and pure water, 2-3 min each.

\subsubsection{X-gal Histochemistry}

$\mathrm{X}$-gal histochemistry in brain sections or whole mount embryos was used for chromogenic detection of $\beta$-galactosidase activity (fig 1e; fig 13; fig 29b). Brain tissue or whole embryos were fixed for maximal $1-2 \mathrm{~h}$ as described in sect 4.7.1. Brains were sectioned using a vibratome or cryostat as described in sect 4.7.2.

The tissue was preincubated for $\sim 15$ min in X-gal rinse solution (tab $5 a$ ) and stained for 1 to $16 \mathrm{~h}$ in X-gal staining solution (tab $5 \mathrm{~b}$ ) at $37^{\circ} \mathrm{C}$ in the dark. During incubation, the staining intensity was monitored from time to time using a bright field microscope. Stained tissue was repeatedly washed for $10 \mathrm{~min}$ in PBS, postfixed for $30 \mathrm{~min}$ in NBF and again washed in PBS. Tissue sections were mounted on glass slides, and coverslips were applied using gelatin or hydrophilic embedding media.

\subsubsection{Myelin Staining}

Gallyas silver impregnation ${ }^{1}$ was used to stain myelin and to visualize axonal fiber tracts in adult brains (fig 17a-f). Paraffin section were deparaffinized using xylene; rehydrated in an descending series of aqueous ethanol solutions; washed

\footnotetext{
${ }^{1}$ Gallyas 1979: "For staining myelin with silver a physical development technique has been devised that can render visible the thinnest fibers [...] even in the early phase of myelination."
} 
a

\begin{tabular}{l|r|r} 
Substance & Amount & Concentration \\
\hline potassium ferricyanide & $1646 \mathrm{mg}$ & $5 \mathrm{mM}$ \\
potassium ferrocyanide & $2112 \mathrm{mg}$ & $5 \mathrm{mM}$ \\
$\mathrm{MgCl}_{2}$ & $407 \mathrm{mg}$ & $2 \mathrm{mM}$ \\
sodium deoxycholic acid & $100 \mathrm{mg}$ & $0.01 \%$ \\
NP40 & $200 \mu \mathrm{l}$ & $0.02 \%$ \\
PBS & ad $1000 \mathrm{ml}$ &
\end{tabular}

b

\begin{tabular}{l|r|r} 
Substance & Amount & Concentration \\
\hline X-gal $(20 \mathrm{mg} / \mathrm{ml}$ in DMSO) & $600 \mu \mathrm{l}$ & $1 \mathrm{mg} / \mathrm{ml}$ \\
X-gal rinse solution & ad $10 \mathrm{ml}$ &
\end{tabular}

Table 5: X-gal staining solution

(a) X-gal rinse and stock solution. (b) X-gal staining solutions (should be freshly prepared).

three times in water; incubated in a solution of pyridine and acetic acid anhydride $(2: 1)$ for $30 \mathrm{~min}$; washed in water for $10 \mathrm{~min}$; incubated in a solution of $0.1 \%$ ammonium nitrate, $0.1 \%$ silver nitrate and $0.01 \%$ sodium hydroxide $(\mathrm{pH} 7.5)$ in water for $10 \mathrm{~min}$ at $30^{\circ} \mathrm{C}$; rinsed three times in $0.5 \%$ acetic acid in water; developed in a fresh solution of $1.75 \%$ sodium carbonate, $0.13 \%$ ammonium nitrate, $0.13 \%$ silver nitrate, $0.65 \%$ tungsten silicic acid and $0.13 \%$ formalin in water for 1-5 min depending on the desired staining intensity; rinsed three times in $0.5 \%$ acetic acid in water; washed in water; incubated in $2 \%$ sodium thiosulfate for $5 \mathrm{~min}$; washed in water for $5 \mathrm{~min}$; dehydrated in an ascending ethanol series followed by xylene; and mounted on glass slides using Eukitt.

\subsubsection{Immunohistochemistry}

To retrieve protein epitopes that had been masked during formalin fixation and dehydration, ${ }^{1}$ deparaffinized paraffin sections (sect 4.7 .2 .3 ) were pretreated by continuous boiling in either citric buffer $(1.5 \mathrm{mM}$ citric acid, $9 \mathrm{mM}$ sodium citrate, pH 6) or Tris EDTA (10 mM Tris Base, 1 mM EDTA, $0.05 \%$ Tween 20, pH 9) for 10-45 min.

Pretreated paraffin sections or fresh cryostat sections were washed in PBS containing 1-2 \% bovine serum albumin (PBS/BSA) or Tris buffer (0.05 M Tris, $0.9 \% \mathrm{NaCl}, \mathrm{pH}$ 9) containing $2 \%$ milk powder (Tris+); blocked in $20 \%$ goat serum in PBS/BSA or Tris+ for $1 \mathrm{~h}$; incubated with primary antibodies (tab 6) in $5 \%$ goat serum in PBS/BSA or Tris+ for $2 \mathrm{~h}$ at RT or overnight at $4^{\circ} \mathrm{C}$; and washed at least 3 times for 5 min in PBS/BSA or Tris+.

For fluorescent detection and protein co-localization studies, sections were incubated with appropriate fluorescently conjugated secondary antibodies for $2 \mathrm{~h}$

\footnotetext{
${ }^{1}$ Fowler et al. 2011: This paper discusses five different models of formalin fixation- and dehydration-induced epitope modifications and uses formalin treated model proteins to characterize changes in conformation and thermal stability.
} 
at RT or overnight at $4^{\circ} \mathrm{C}$; counterstained using 4',6-diamidino-2-phenylindole (DAPI) in PBS (1:5000); washed 3 times in PBS; quickly rinsed in water; and mounted in aqueous mounting media containing fluorescence protecting agents (Polyscience Aqua-Poly/Mount or DAKO Fluorescence Mounting Medium).

For standard chromogenic detection, sections were incubated for 3 min in $3 \%$ hydrogen peroxide to inactivate endogenous peroxidases; washed twice in PBS or Tris buffer; rinsed in PBS/BSA or Tris+; incubated with appropriate biotinylated secondary antibodies in PBS/BSA or Tris+ for $20 \mathrm{~min}$; washed 3 times in PBS or Tris buffer, incubated with horseradish peroxidase (HRP)-coupled streptavidin in PBS for 10 min, washed 3 times in PBS; incubated with diaminobenzidine (DAB) in Tris buffer for 10 min to form a brown precipitate in the presence of HRP; washed in PBS; dehydrated by sequential incubation in water, $50 \%$ ethanol, $70 \%$ ethanol, $90 \%$ ethanol, $100 \%$ ethanol, isopropanol/xylene (1:1) and xylene, 2-3 min each; and mounted using Eukitt.

For highly sensitive chromogenic detection, sections were incubated with appropriate alkaline phosphatase (AP)-coupled secondary antibodies in PBS/BSA for 30 min; washed 3 times in PBS; incubated in NTB/BCIP to form a blue precipitate in the presence of AP or alternatively in Fast Red TR/Naphthol ${ }^{1}$ to form a red precipitate in the presence of AP for 30 min up to $48 \mathrm{~h}$, depending on the desired signal intensity; and further processed as described in the above paragraph.

\subsubsection{In Situ Hybridization}

Fresh cryostat sections (sect 4.7.2.2) were postfixed in $4 \%$ paraformaldehyde (PFA) in PBS containing $0.1 \%$ diethyl pyrocarbonate (DEPC) for $15 \mathrm{~min}$. Sections were immediately washed in PBS-DEPC, $\mathrm{pH} 7.4$ and incubated with $20 \mathrm{~g} / \mathrm{ml}$ Proteinase $\mathrm{K}$ in $20 \mathrm{mM}$ Tris, $\mathrm{pH} 7.5$, and $1 \mathrm{mM}$ EDTA, $\mathrm{pH} 8$, for $2.5 \mathrm{~min}$ at $37^{\circ} \mathrm{C}$. Proteinase $\mathrm{K}$ was inactivated in $0.2 \%$ glycine in PBS-DEPC; the sections were washed in PBS-DEPC; again postfixed in $4 \%$ PFA and $0.2 \%$ glutaraldehyde in PBS-DEPC for 20 min; washed in PBS-DEPC; and prehybridized in hybridization buffer (tab 7) at $70^{\circ} \mathrm{C}$ for $2 \mathrm{~h}$. RNA hybridization was performed overnight in hybridization buffer (tab 7) containing digoxigenin (DIG)-labeled and heatdenatured cRNA probe at $70^{\circ} \mathrm{C}$ in a sealed humid chamber filled with $50 \%$ formamide. On the next day, slides were rinsed in $2 \times \mathrm{SSC}, \mathrm{pH} 4.5$; treated with $20 \mathrm{~g} / \mathrm{ml}$ RNase in $0.5 \mathrm{M} \mathrm{NaCl} / 10 \mathrm{mM}$ Tris for $30 \mathrm{~min}$ at $37^{\circ} \mathrm{C}$; rinsed in $2 \times \mathrm{SSC}, \mathrm{pH} 4.5$; washed three times in $50 \%$ formamide in $2 \times \mathrm{SSC}, \mathrm{pH} 4.5$, for $30 \mathrm{~min}$ at $65^{\circ} \mathrm{C}$; rinsed in $50 \mathrm{mM}$ Tris, pH 7.5, $150 \mathrm{mM} \mathrm{NaCl}, 10 \mathrm{mM} \mathrm{KCl}$, $1 \%$ Triton-X (KTBT); blocked in $20 \%$ sheep serum in KTBT (KTBT+S) for $2 \mathrm{~h}$; incubated with anti-DIG alkaline phosphatase coupled antibody (Roche) at 1:1000 in $\mathrm{KTBT}+\mathrm{S}$ overnight at $4^{\circ} \mathrm{C}$; washed four times in KTBT for $30 \mathrm{~min}$; and rinsed in $100 \mathrm{mM}$ Tris, pH 9.5, $100 \mathrm{mM} \mathrm{NaCl}, 50 \mathrm{mM} \mathrm{MgCl}_{2}, 0.05 \%$ Tween 20 (NTMT). The chromogenic staining reaction was performed by incubating the sections for $1-48 \mathrm{~h}$ in NBT/BCIP (Roche) at $37^{\circ} \mathrm{C}$. The staining intensity was periodically monitored using a bright field microscope. Stained sections were washed in PBS;

\footnotetext{
${ }^{1}$ Murdoch et al. 1990
} 


\begin{tabular}{l|l|l|l|l|l} 
Target & Class & Host & Supplier & Tissue & Dilution \\
\hline Blbp & pc & rabbit & Chemicon & $\mathrm{P}$ & $1: 500$ \\
Brn2 & pc & goat & Santa Cruz & $\mathrm{P}$ & $1: 200$ \\
Calb2 & pc & rabbit & Chemicon & $\mathrm{P}$ & $1: 200$ \\
Cnp & IgG & mouse & Sigma & $\mathrm{P}$ & $1: 150$ \\
Cntn2 & IgM & mouse & DSHB & $\mathrm{V}$ & $1: 200$ \\
Cre & pc & rabbit & Babco & $\mathrm{P}^{\star}, \mathrm{V}, \mathrm{C}$ & $1: 2000$ \\
Ctip2 & IgG & rat & AbCam & $\mathrm{P}$ & $1: 500$ \\
Fnp7 & IgG & mouse & Zymed & $\mathrm{P}$ & $1: 200$ \\
Foxp2 & pc & rabbit & AbCam & $\mathrm{P}$ & $1: 400$ \\
Gap43 & IgG & mouse & Sigma, G9264 & $\mathrm{P}$ & $1: 200$ \\
Gfap & IgG & mouse & Novacastra, GA5 & $\mathrm{P}$ & $1: 200$ \\
L1cam & pc & rat & Chemicon & $\mathrm{C}$ & $1: 500$ \\
Lmo4 & & rat & J. E. Visvader & $\mathrm{P}$ & $1: 500$ \\
Map2 (a+b) & IgG & mouse & Millipore, MAB378 & $\mathrm{P}, \mathrm{V}, \mathrm{C}$ & $1: 200$ \\
Mbp & IgG & mouse & DAKO & $\mathrm{P}$ & $1: 1000$ \\
NeuN & IgG & mouse & Chemicon & $\mathrm{P}, \mathrm{C}$ & $1: 200$ \\
Pcna & pc & rabbit & AbCam & $\mathrm{P}$ & $1: 200$ \\
Reelin & IgG & mouse & Chemicon & $\mathrm{P}$ & $1: 400$ \\
Robo1 & pc & rabbit & F. Murakami & $\mathrm{P}$ & $1: 2000$ \\
Satb2 & pc & rabbit & V. Tarabykin & $\mathrm{P}$ & $1: 2000$ \\
Sox5 & pc & goat & R\&D Systems & $\mathrm{P}$ & $1: 500$ \\
Tbr1 & pc & rabbit & AbCam & $\mathrm{P}$ & $1: 200$ \\
Vglut1 & pc & rabbit & SySys & $\mathrm{P}$ & $1: 400$
\end{tabular}

Table 6: Overview of primary antibodies

Column headers: Target, Gene symbol of the detected protein; Class, Immunoglobulin isotype of monoclonal antibodies or 'pc' for polyclonal sera; Host, Species the antibody was raised in; Tissue, Antibody was successfully used in tissue processed for V: vibratome, C: cryostat, or $\mathbf{P}$ : paraffin sectioning (* only successful after extensive antigen unmasking); Dilution, Supplied antibody solutions were diluted for final use.

postfixed in NBF for 30 min; washed in PBS containing $0.1 \%$ Triton-X; rinsed in PBS; dehydrated by sequential incubation in water, $50 \%$ ethanol, $70 \%$ ethanol, $90 \%$ ethanol, $100 \%$ ethanol, isopropanol/xylene (1:1) and xylene, 2-3 min each; cleared in a solution of benzyl benzoate and benzyl alcohol (2:1); and mounted using Eukitt.

\subsection{Software}

Free and open source software, and open file formats were used whenever possible. My own computer systems and all software were managed using 


\begin{tabular}{l|r|r} 
a Substance & Amount & Concentration \\
\hline RNAse free water (DEPC treated) & $8 \mathrm{ml}$ & $20 \%$ \\
Formamide (deionized) & $20 \mathrm{ml}$ & $50 \%$ \\
$20 \times$ SSC, ph 4.5 & $10 \mathrm{ml}$ & $5 \times$ \\
Blocking reagent (Roche) & $400 \mathrm{mg}$ & $1 \times$ \\
\hline $0.2 \mathrm{M} \mathrm{EDTA}$ & $1 \mathrm{ml}$ & $5 \mathrm{mM}$ \\
Tween 20 & $40 \mu \mathrm{l}$ & $0.1 \%$ \\
$10 \%$ CAPS & $400 \mu \mathrm{l}$ & $0.1 \%$ \\
$100 \mathrm{mg} / \mathrm{ml}$ heparin & $40 \mu \mathrm{l}$ & $0.1 \mathrm{mg} / \mathrm{ml}$ \\
$10 \mathrm{mg} / \mathrm{ml}$ yeast RNA & $200 \mu \mathrm{l}$ & $100 \mu \mathrm{g} / \mathrm{ml}$ \\
\hline & $40 \mathrm{ml}$ &
\end{tabular}

Table 7: ISH In situ hybridization buffer

Preparation: Water, formamide, SSC and blocking reagent are mixed and heated to $70^{\circ} \mathrm{C}$ until the solution turns clear. Tube is put on ice and all other substances are added. Buffer can be stored at $-20^{\circ} \mathrm{C}$.

Debian $^{1}$ and NixOS ${ }^{2}$ Gnu/Linux distributions. All data and manuscript files were automatically backed up and synchronized between different computer networks using Unison ${ }^{3}$. Computer code that was written for this study is available on request.

\subsubsection{Statistical Analysis}

The following applies unless stated otherwise in the text. Data processing and statistical analysis were performed using $\mathrm{R}^{4}$ and Gnumeric ${ }^{5}$. Values are expressed as arithmetic mean \pm standard error of the mean (SEM). To determine significance, $p$-values were calculated using unpaired, two-tailed student's t-tests (function t.test with standard parameters in $R$ ). Local regression curves were calculated using linear least squares (function loess.smooth with parameters family=gaussian and span=0.35 in $R$ ). Statistical visualizations were automatically generated from data using $R$ scripts.

\footnotetext{
${ }^{1}$ Debian is one of the earliest Linux distributions, it was originally developed by Ian Murdock (Scheetz 1998). http://www.debian.org/

${ }^{2}$ NixOS is a purely functional Linux distribution developed at Universiteit Utrecht in the Netherlands (Dolstra 2003, 2006). http://nixos.org/

${ }^{3}$ Unison is a bidirectional cross-platform file-synchronization tool written in Ocaml (Pierce and Vouillon 2004). http://www.cis. upenn.edu/ bcpierce/unison/ (GPL)

${ }^{4} R$ is a statistical programming language that was originally written by Robert Gentleman and Ross Ihaka at the University of Auckland, USA (R Development Core Team 2008). It is an alternative implementation of the similar language $S$ by Bell Laboratories. http://r-project.org (GPL)

${ }^{5}$ Gnumeric is a spreadsheet software (Keeling and Pavur 2011). http://gnumeric.org (GPL)
} 


\subsubsection{Sequence Alignment}

Sequences were downloaded from the $\mathrm{NCBI}^{1}$ website and processed using $A p E .^{2}$ Multiple sequences alignment was calculated using ClustalX ${ }^{3}$ (with standard parameters). Rooted phylogenetic trees were generated using using the UPGMA algorithm (Unweighted Pair Group Method with Arithmetic mean) of PHYLIP (sect 1.3.1).

\subsubsection{Genetic Modeling}

The ability of recurrent transcription factor networks to generate sharp borders of mutually exclusive expression domains (fig 3d-f) was demonstrated using discrete simulations of minimal genetic networks. The model was implemented in Ocaml. ${ }^{5}$ Cairo ${ }^{6}$ was used for the visualization of expression pattern (sect 1.2.2). Directed graphs of the simulated genetic networks were rendered using Graphviz. ${ }^{7}$

\subsubsection{Image Processing}

Digital images of stained tissue sections were obtained using Axiophot (Zeiss, Germany) or DM RXA (Leica, Germany) microscopes equipped with DCC cameras (Kappa, Germany). Images were processed using GIMP ${ }^{8}$ or ImageJ $^{9}$. Image stacks were processed using ImageMagick ${ }^{10}$ and the TurboReg/StackReg ${ }^{11}$ plugins for ImageJ. Protein crystal structure data obtained from the NCBI retrieval service was visualized using $C n 3 D^{12}$ and Avogadro ${ }^{13}$.

\footnotetext{
${ }^{1}$ National Center for Biotechnology Information (NCBI) sequence database (mouse genes): http://ncbi.nlm.nih.gov/gene?cmd=search\&term=Mus+musculus [orgn]

${ }^{2} A p E$ is 'A plasmid Editor' by M. Wayne Davis. http://www.biology.utah.edu/jorgensen/wayned/ape/

${ }^{3}$ Clustal is a software package for multiple sequence alignment. http://clustal.org (LGPL)

${ }^{4} P H Y L I P$ is a collection of programs for inferring phylogenies (Felsenstein 1981). http://evolution.genetics. washington.edu/phylip.html (BSD)

${ }^{5}$ Ocaml (Objective Categorical Abstract Machine Language) is a statically type-interfering multiparadigm programming language and compiler initially developed by Xavier Leroy at the Institut National de Recherche en Informatique et en Automatique in France (Leroy 1990): http://ocaml .org (LGPL, QPL)

${ }^{6}$ Cairo is a popular graphics library with bindings for Ocaml. http://cairographics .org/cairo-ocaml/ (LGPL, MPL)

${ }^{7}$ Graphviz is a tool set for graph visualization originally developed by AT\&T http://www.graphviz.org/ (EPL)

${ }^{8}$ GIMP, the Gnu Image Manipulation Program. http://gimp.org (GPL)

${ }^{9}$ ImageJ is a suite of scientific image processing and analysis software (Schneider et al. 2012). http://rsb.info.nih.gov/ij (PD)

${ }^{10}$ ImageMagick is a collection of non-interactive programs for bitmap image manipulation. http://imagemagick.org (AL)

${ }^{11}$ TurboReg and StackReg are ImageJ plugins for the recursive alignment of image stacks (Thévenaz et al. 1998): http://bigwww.epfl.ch/thevenaz/turboreg and http://bigwww.epfl.ch/thevenaz/stackreg

${ }^{12} \mathrm{Cn} 3 \mathrm{D}$ is a viewer for macromolecular structure data (Wang et al. 2000). http://ncbi.nlm.nih.gov/Structure/CN3D/cn3d.shtml (PD)

${ }^{13}$ Avogadro is a viewer and editor for molecular structure data (Hanwell et al. 2012). http://avogadro.cc (GPL)
} 


\subsubsection{Manuscript Preparation}

The manuscript was edited in $L_{Y} X^{1}$ and typesetted using $\operatorname{LAT}_{E} \mathrm{X}^{2}\left(\mathrm{~T}_{\mathrm{E}} \mathrm{X}\right.$ Live ${ }^{3}$ ) and KOMA-Script. ${ }^{4}$ References were retrieved and managed using Zotero $^{5}$ (in combination with a local WebDAV server ${ }^{6}$ ) and converted to BibTEX format for use in $L_{Y} X / L T_{E} X$. Figures composites were assembled in Scribus. ${ }^{7}$ Schemata were drawn using Inkscape ${ }^{8}$.

${ }^{1} L_{Y} X$ is a visual $E T_{E} X$-based document processor. http://lyx.org (GPL)

${ }^{2} L T_{E} X$ is a document preparation system that was initially developed by Leslie Lamport as macro package extending $T_{E} X$ by Donald E. Knuth at Stanford University in California, USA (Knuth 1986; Lamport 1994; Downes 2002). http://latex-project.org (LPPL)

${ }^{3} T_{E} X$ Live is an extensive distribution of $\left[A T_{E} X\right.$ packages. http://tug.org/texlive

${ }^{4}$ KOMA-Script is a collection of internationalized $\mathrm{AT}_{\mathrm{E}} \mathrm{X}$ document classes (Kohm 2014). http://komascript.de (LPPL)

${ }^{5}$ Zotero is a reference management software developed at George Mason University in Fairfax, Virginia, USA.

http://zotero.org (AGPL)

${ }^{6}$ mod_dav is a WebDAV module for the Apache web server. http://httpd.apache.org/docs/current/mod/mod_dav.html

${ }^{7}$ Scribus is a desktop publishing (DTP) system. http://scribus.net (GPL)

${ }^{8}$ Inkscape is a visual editor for scalable vector graphics (SVG) files. http://inkscape.org (GPL) 


\section{Bibliography}

Aboitiz F and Montiel J (2007): Co-option of signaling mechanisms from neural induction to telencephalic patterning. Rev Neurosci 18 (3-4), 311-342. Cited on $\mathrm{p} 7$.

Agarwal A, Dibaj P, Kassmann CM, Goebbels S, Nave KA and Schwab MH (2012): In vivo imaging and noninvasive ablation of pyramidal neurons in adult NEX-CreERT2 mice. Cereb Cortex 22 (7), 1473-1486. Cited on p 30 and 89.

Alcamo EA, Chirivella L, Dautzenberg M, Dobreva G, Fariñas I, Grosschedl R and McConnell SK (2008): Satb2 regulates callosal projection neuron identity in the developing cerebral cortex. Neuron 57 (3), 364-77. Cited on p 41.

Allen-Brady K, Miller J, Matsunami N, Stevens J, Block H, Farley M, Krasny L, Pingree C, Lainhart J, Leppert M et al. (2009): A high-density SNP genome-wide linkage scan in a large autism extended pedigree. Mol Psychiatry 14 (6), 590-600. Cited on p 46.

Andrews W, Liapi A, Plachez C, Camurri L, Zhang J, Mori S, Murakami F, Parnavelas JG, Sundaresan V and Richards LJ (2006): Robol regulates the development of major axon tracts and interneuron migration in the forebrain. Development 133 (11), 22432252. Cited on $\mathrm{p} 12$ and 50.

Angevine JB and Sidman RL (1961): Autoradiographic study of cell migration during histogenesis of cerebral cortex in the mouse. Nature 192, 766-768. Cited on p 10.

Arimura N and Kaibuchi K (2007): Neuronal polarity: from extracellular signals to intracellular mechanisms. Nat Rev Neurosci 8 (3), 194-205. Cited on p 11.

Arlotta P, Molyneaux BJ, Chen J, Inoue J, Kominami R and Macklis JD (2005): Neuronal subtype-specific genes that control corticospinal motor neuron development in vivo. Neuron 45 (2), 207-21. Cited on $\mathrm{p} 41$.

Armentano M, Filosa A, Andolfi G and Studer M (2006): COUP-TFI is required for the formation of commissural projections in the forebrain by regulating axonal growth. Development 133 (21), 4151-4162. Cited on p 84.

Armentano M, Chou SJ, Tomassy GS, Leingärtner A, O'Leary DDM and Studer M (2007): COUP-TFI regulates the balance of cortical patterning between frontal/motor and sensory areas. Nat Neurosci 10 (10), 1277-1286. Cited on p 8.

Arnold SJ, Huang GJ, Cheung AFP, Era T, Nishikawa SI, Bikoff EK, Molnár Z, Robertson EJ and Groszer M (2008): The T-box transcription factor Eomes/Tbr2 regulates neurogenesis in the cortical subventricular zone. Genes Dev 22 (18), 2479-2484. Cited on p 36.

Asprer JST, Lee B, Wu CS, Vadakkan T, Dickinson ME, Lu HC and Lee SK (2011): LMO4 functions as a co-activator of neurogenin 2 in the developing cortex. Development 138 (13), 2823-2832. Cited on $\mathrm{p} 74$.

Assimacopoulos S, Grove EA and Ragsdale CW (2003): Identification of a Pax6dependent epidermal growth factor family signaling source at the lateral edge of the embryonic cerebral cortex. J Neurosci 23 (16), 6399-6403. Cited on p 7.

Atchley WR and Fitch WM (1997): A natural classification of the basic helix-loop-helix class of transcription factors. Proc Natl Acad Sci U S A 94 (10), 5172-5176. Cited on p 15.

Autism Genome Project Consortium, Szatmari P, Paterson AD, Zwaigenbaum L, Roberts W, Brian J, Liu XQ, Vincent JB, Skaug JL, Thompson AP et al. (2007): Mapping autism risk loci using genetic linkage and chromosomal rearrangements. Nat Genet 39 (3), 319328. Cited on $\mathrm{p} 46$. 
Azevedo FAC, Carvalho LRB, Grinberg LT, Farfel JM, Ferretti REL, Leite REP, Jacob Filho W, Lent R and Herculano-Houzel S (2009): Equal numbers of neuronal and nonneuronal cells make the human brain an isometrically scaled-up primate brain. $\mathrm{J}$ Comp Neurol 513 (5), 532-541. Cited on p 3.

Azim E, Shnider SJ, Cederquist GY, Sohur US and Macklis JD (2009): Lmo4 and Clim1 progressively delineate cortical projection neuron subtypes during development. Cereb Cortex 19 Suppl 1, i62-69. Cited on p 41.

Bagri A, Marín O, Plump AS, Mak J, Pleasure SJ, Rubenstein JLR and Tessier-Lavigne M (2002): Slit proteins prevent midline crossing and determine the dorsoventral position of major axonal pathways in the mammalian forebrain. Neuron 33 (2), 233-48. Cited on $\mathrm{p} 83$.

Baker NE (2001): Master regulatory genes; telling them what to do. Bioessays 23 (9), 763-766. Cited on $\mathrm{p} 16$.

Ballas N, Grunseich C, Lu DD, Speh JC and Mandel G (2005): REST and its corepressors mediate plasticity of neuronal gene chromatin throughout neurogenesis. Cell 121 (4), 645-657. Cited on p 91.

Bar-Yam Y, Harmon D and de Bivort B (2009): Systems biology. Attractors and democratic dynamics. Science 323 (5917), 1016-1017. Cited on p 17.

Baranek C, Dittrich M, Parthasarathy S, Bonnon CG, Britanova O, Lanshakov D, Boukhtouche F, Sommer JE, Colmenares C, Tarabykin V et al. (2012): Protooncogene Ski cooperates with the chromatin-remodeling factor Satb2 in specifying callosal neurons. Proc Natl Acad Sci USA 109 (9), 3546-3551. Cited on p 41.

Barco A and Marie H (2011): Genetic approaches to investigate the role of CREB in neuronal plasticity and memory. Mol Neurobiol 44 (3), 330-349. Cited on p 91.

Bartholomä A and Nave KA (1994): NEX-1: a novel brain-specific helix-loop-helix protein with autoregulation and sustained expression in mature cortical neurons. Mech Dev 48 (3), 217-28. Cited on p 19 and 28.

Bashaw GJ and Klein R (2010): Signaling from Axon Guidance Receptors. Cold Spring Harb Perspect Biol 2 (5). Cited on p 70.

Baum EB: What Is Thought? MIT Press, Cambridge, MA 2004. Cited on $\mathrm{p} 3$ and 73.

Bear MF, Connors BW and Paradiso MA: Neuroscience: exploring the brain. Williams \& Wilkins, Baltimore 1996. Cited on p 6.

Bedi KS, Thomas YM, Davies CA and Dobbing J (1980): Synapse-to-neuron ratios of the frontal and cerebellar cortex of 30-day-old and adult rats undernourished during early postnatal life. J Comp Neurol 193 (1), 49-56. Cited on p 3.

Bertrand N, Castro DS and Guillemot F (2002): Proneural genes and the specification of neural cell types. Nat Rev Neurosci 3 (7), 517-530. Cited on p 17.

Bielle F, Griveau A, Narboux-Nême N, Vigneau S, Sigrist M, Arber S, Wassef M and Pierani A (2005): Multiple origins of Cajal-Retzius cells at the borders of the developing pallium. Nat Neurosci 8 (8), 1002-1012. Cited on $\mathrm{p} 10$.

Bishop KM, Garel S, Nakagawa Y, Rubenstein JLR and O'Leary DDM (2003): Emx1 and Emx2 cooperate to regulate cortical size, lamination, neuronal differentiation, development of cortical efferents, and thalamocortical pathfinding. J Comp Neurol 457 (4), 345-60. Cited on $\mathrm{p} 84$.

Blake JA, Bult CJ, Eppig JT, Kadin JA, Richardson JE and Mouse Genome Database Group (2014): The Mouse Genome Database: integration of and access to knowledge about the laboratory mouse. Nucleic Acids Res 42 (Database issue), D810-817. Cited on $\mathrm{p} 132$.

Bock HH and Herz J (2003): Reelin activates SRC family tyrosine kinases in neurons. Curr Biol 13 (1), 18-26. Cited on p 81. 
Boeckx C and Benítez-Burraco A (2014): Globularity and language-readiness: generating new predictions by expanding the set of genes of interest. Front Psychol 5, 1324. Cited on $\mathrm{p} 84$.

Borello U and Pierani A (2010): Patterning the cerebral cortex: traveling with morphogens. Curr Opin Genet Dev 20 (4), 408-415. Cited on p 8 and 9.

Bormuth I, Yan K, Yonemasu T, Gummert M, Zhang M, Wichert S, Grishina O, Pieper A, Zhang W, Goebbels S et al. (2013): Neuronal basic helix-loop-helix proteins Neurod2/6 regulate cortical commissure formation before midline interactions. J Neurosci 33 (2), 641-651. Cited on p 18, 20, 21, 23, 30, 31, 49, 51, 93, 96, and 129.

Britanova O, Akopov S, Lukyanov S, Gruss P and Tarabykin V (2005): Novel transcription factor Satb2 interacts with matrix attachment region DNA elements in a tissue-specific manner and demonstrates cell-type-dependent expression in the developing mouse CNS. Eur J Neurosci 21 (3), 658-668. Cited on p 41.

Britanova O, de Juan Romero C, Cheung A, Kwan KY, Schwark M, Gyorgy A, Vogel T, Akopov S, Mitkovski M, Agoston D et al. (2008): Satb2 is a postmitotic determinant for upper-layer neuron specification in the neocortex. Neuron 57 (3), 378-92. Cited on p 41 and 130 .

Brock LG, Coombs JS and Eccles JC (1952): The recording of potentials from motoneurones with an intracellular electrode. J Physiol 117 (4), 431-460. Cited on $\mathrm{p} 4$.

Bröhl D, Strehle M, Wende H, Hori K, Bormuth I, Nave KA, Müller T and Birchmeier C (2008): A transcriptional network coordinately determines transmitter and peptidergic fate in the dorsal spinal cord. Dev Biol 322 (2), 381-393. Cited on p 69 and 72.

Brzózka MM, Radyushkin K, Wichert SP, Ehrenreich H and Rossner MJ (2010): Cognitive and sensorimotor gating impairments in transgenic mice overexpressing the schizophrenia susceptibility gene Tcf4 in the brain. Biol Psychiatry 68 (1), 33-40. Cited on $\mathrm{p} 92$.

Bulfone A, Smiga SM, Shimamura K, Peterson A, Puelles L and Rubenstein JL (1995): Tbrain-1: a homolog of Brachyury whose expression defines molecularly distinct domains within the cerebral cortex. Neuron 15 (1), 63-78. Cited on p 36.

Bull SJ: Mechanisms Promoting Myelin Formation and Maintenance Under Normal and Pathological Conditions. Biol. Diss. Montreal, Canada, Montreal, Canada 2011. Cited on p 59.

Cajal SRy: Textura del Sistema Nervioso del Hombre y de los Vertebrados. Zaragoza, Madrid 1904. Cited on $\mathrm{p} 4$ and 5.

Carter TA, Greenhall JA, Yoshida S, Fuchs S, Helton R, Swaroop A, Lockhart DJ and Barlow C (2005): Mechanisms of aging in senescence-accelerated mice. Genome Biol 6 (6), R48. Cited on p 92.

Cassimeris L and Spittle C (2001): Regulation of microtubule-associated proteins. Int Rev Cytol 210, 163-226. Cited on p 37.

Chan CH, Godinho LN, Thomaidou D, Tan SS, Gulisano M and Parnavelas JG (2001): Emx1 is a marker for pyramidal neurons of the cerebral cortex. Cereb Cortex 11 (12), 1191-8. Cited on $\mathrm{p} 84$.

Chan SSK and Kyba M (2013): What is a Master Regulator? J Stem Cell Res Ther $\underline{3}$. Cited on $\mathrm{p} 16$.

Charron F and Tessier-Lavigne M (2005): Novel brain wiring functions for classical morphogens: A role as graded positional cues in axon guidance. Development 132 (10), 2251-2262. Cited on $\mathrm{p} 12$.

Chen B, Schaevitz LR and McConnell SK (2005): Fezl regulates the differentiation and axon targeting of layer 5 subcortical projection neurons in cerebral cortex. Proc Natl Acad Sci USA 102 (47), 17184-9. Cited on p 42. 
Chen B, Wang SS, Hattox AM, Rayburn H, Nelson SB and McConnell SK (2008): The Fezf2-Ctip2 genetic pathway regulates the fate choice of subcortical projection neurons in the developing cerebral cortex. Proc Natl Acad Sci U S A 105 (32), 11382-7. Cited on $\mathrm{p} 42$.

Chen Y, Magnani D, Theil T, Pratt T and Price DJ (2012): Evidence that descending cortical axons are essential for thalamocortical axons to cross the pallial-subpallial boundary in the embryonic forebrain. PLoS ONE 7 (3), e33105. Cited on p 70 and 71.

Cherry TJ, Wang S, Bormuth I, Schwab M, Olson J and Cepko CL (2011): NeuroD factors regulate cell fate and neurite stratification in the developing retina. J Neurosci 31 (20), 7365-7379. Cited on $\mathrm{p} 72$.

Chiaramello A, Neuman T, Peavy DR and Zuber MX (1996): The GAP-43 gene is a direct downstream target of the basic helix-loop-helix transcription factors. J Biol Chem 271 (36), 22035-22043. Cited on p 46.

Chizhikov VV and Millen KJ (2005): Roof plate-dependent patterning of the vertebrate dorsal central nervous system. Dev Biol 277 (2), 287-295. Cited on p 6.

Cho JH and Tsai MJ (2006): Preferential posterior cerebellum defect in BETA2/NeuroD1 knockout mice is the result of differential expression of BETA2/NeuroD1 along anteriorposterior axis. Dev Biol 290 (1), 125-138. Cited on p 85.

Cho JH, Kwon IS, Kim S, Ghil SH, Tsai MJ, Kim YS, Lee YD and Suh-Kim H (2001): Overexpression of BETA2/NeuroD induces neurite outgrowth in F11 neuroblastoma cells. J Neurochem 77 (1), 103-109. Cited on p 19.

Cohen-Tannoudji M, Babinet C and Wassef M (1994): Early determination of a mouse somatosensory cortex marker. Nature 368 (6470), 460-463. Cited on p 81.

Coons AH, Creech HJ, Jones RN and Berliner E (1942): The Demonstration of Pneumococcal Antigen in Tissues by the Use of Fluorescent Antibody. J Immunol 45 (3), 159-170. Cited on $\mathrm{p} 4$.

Cooper JA (2013): Cell biology in neuroscience: mechanisms of cell migration in the nervous system. J Cell Biol 202 (5), 725-734. Cited on p 76.

Cooper JA (2014): Molecules and mechanisms that regulate multipolar migration in the intermediate zone. Front Cell Neurosci 8, 386. Cited on p 78 and 80.

Cowan WM: Molecular and Cellular Approaches to Neural Development. Oxford Univ Pr 1998. Cited on $\mathrm{p} 3$ and 93.

Crick FH, Barnett L, Brenner S and Watts-Tobin RJ (1961): General nature of the genetic code for proteins. Nature 192, 1227-1232. Cited on $\mathrm{p} 4$.

Cubelos B, Sebastián-Serrano A, Beccari L, Calcagnotto ME, Cisneros E, Kim S, Dopazo A, Alvarez-Dolado M, Redondo JM, Bovolenta P et al. (2010): Cux1 and Cux2 regulate dendritic branching, spine morphology, and synapses of the upper layer neurons of the cortex. Neuron 66 (4), 523-535. Cited on p 17.

D'Amico LA, Boujard D and Coumailleau P (2013): The neurogenic factor NeuroD1 is expressed in post-mitotic cells during juvenile and adult Xenopus neurogenesis and not in progenitor or radial glial cells. PLoS ONE 8 (6), e66487. Cited on p 88.

Daniels RW, Collins CA, Chen K, Gelfand MV, Featherstone DE and DiAntonio A (2006): A single vesicular glutamate transporter is sufficient to fill a synaptic vesicle. Neuron 49 (1), 11-16. Cited on p 54.

D’Arcangelo G, Miao GG, Chen SC, Soares HD, Morgan JI and Curran T (1995): A protein related to extracellular matrix proteins deleted in the mouse mutant reeler. Nature 374 (6524), 719-723. Cited on p 81.

Dehmelt L and Halpain S (2005): The MAP2/Tau family of microtubule-associated proteins. Genome Biol 6 (1), 204. Cited on p 37. 
Demyanenko GP, Tsai AY and Maness PF (1999): Abnormalities in neuronal process extension, hippocampal development, and the ventricular system of L1 knockout mice. J Neurosci 19 (12), 4907-20. Cited on p 83.

Dent EW and Gertler FB (2003): Cytoskeletal dynamics and transport in growth cone motility and axon guidance. Neuron 40 (2), 209-227. Cited on p 11.

Dent EW and Meiri KF (1992): GAP-43 phosphorylation is dynamically regulated in individual growth cones. J Neurobiol 23 (8), 1037-1053. Cited on p 46.

Dickinson E: The Complete Poems of Emily Dickinson. CreateSpace Independent Publishing Platform 2013. Cited on $\mathrm{p} 1$ and 140.

Dodd J, Jessell TM and Placzek M (1998): NEUROSCIENCE: The When and Where of Floor Plate Induction. Science 282 (5394), 1654-1657. Cited on p 6.

Dolstra E, Integrating Software Construction and Software Deployment. In Westfechtel B and Hoek Avd, eds., Software Configuration Management, no. 2649 in Lecture Notes in Computer Science, 102-117, Springer Berlin Heidelberg 2003, dOI: 10.1007/3-54039195-9_8. Cited on p 106.

Dolstra E (2006): The purely functional software deployment model. Cited on $\mathrm{p} 106$.

Dominguez MH, Ayoub AE and Rakic P (2013): POU-III transcription factors (Brn1, $B r n 2$, and Oct6) influence neurogenesis, molecular identity, and migratory destination of upper-layer cells of the cerebral cortex. Cereb Cortex 23 (11), 2632-2643. Cited on p 64.

Downes M (2002): TeX and LaTeX 2e. Notices Amer Math Soc 49 (11), 1384-1391. Cited on $\mathrm{p} 108$.

Dwyer ND, Manning DK, Moran JL, Mudbhary R, Fleming MS, Favero CB, Vock VM, O'Leary DD, Walsh CA and Beier DR (2011): A forward genetic screen with a thalamocortical axon reporter mouse yields novel neurodevelopment mutants and a distinct emx2 mutant phenotype. Neural Dev $\underline{6}, 3$. Cited on p 72 .

Elston GN (2003): Cortex, cognition and the cell: New insights into the pyramidal neuron and prefrontal function. Cereb Cortex 13 (11), 1124-1138. Cited on p 4.

Englund C, Fink A, Lau C, Pham D, Daza RAM, Bulfone A, Kowalczyk T and Hevner RF (2005): Pax6, Tbr2, and Tbr1 are expressed sequentially by radial glia, intermediate progenitor cells, and postmitotic neurons in developing neocortex. J Neurosci 25 (1), 247-251. Cited on p 36.

Erskine L and McCaig CD (1995): Growth cone neurotransmitter receptor activation modulates electric field-guided nerve growth. Dev Biol 171 (2), 330-339. Cited on p 12.

Fazeli A, Dickinson SL, Hermiston ML, Tighe RV, Steen RG, Small CG, Stoeckli ET, Keino-Masu K, Masu M, Rayburn H et al. (1997): Phenotype of mice lacking functional Deleted in colorectal cancer (Dcc) gene. Nature 386 (6627), 796-804. Cited on p 83.

Feil R, Wagner J, Metzger D and Chambon P (1997): Regulation of Cre recombinase activity by mutated estrogen receptor ligand-binding domains. Biochem Biophys Res Commun 237 (3), 752-757. Cited on p 30.

Feldman JL, Mitchell GS and Nattie EE (2003): Breathing: Rhythmicity, Plasticity, Chemosensitivity. Annu Rev Neurosci 26, 239-266. Cited on p 33.

Felsenstein J (1981): Evolutionary trees from DNA sequences: a maximum likelihood approach. J Mol Evol 17 (6), 368-376. Cited on p 107.

Ferland RJ, Cherry TJ, Preware PO, Morrisey EE and Walsh CA (2003): Characterization of Foxp2 and Foxp $1 \mathrm{mRNA}$ and protein in the developing and mature brain. J Comp Neurol 460 (2), 266-279. Cited on p 42 and 65.

Fertuzinhos S, Li M, Kawasawa YI, Ivic V, Franjic D, Singh D, Crair M and Sestan N (2014): Laminar and temporal expression dynamics of coding and noncoding RNAs in the mouse neocortex. Cell Rep 6 (5), 938-950. Cited on p 88. 
Fishell G and Hanashima C (2008): Pyramidal neurons grow up and change their mind. Neuron 57 (3), 333-338. Cited on p 40.

Fowler CB, Evers DL, O'Leary TJ and Mason JT (2011): Antigen Retrieval Causes Protein Unfolding. J Histochem Cytochem 59 (4), 366-381. Cited on p 103.

Franklin A, Kao A, Tapscott S and Unis A (2001): NeuroD homologue expression during cortical development in the human brain. J Child Neurol 16 (11), 849-853. Cited on p 18.

Friederici AD, von Cramon DY and Kotz SA (2007): Role of the corpus callosum in speech comprehension: interfacing syntax and prosody. Neuron 53 (1), 135-145. Cited on p 84.

Fukamauchi F, Aihara O, Wang YJ, Akasaka K, Takeda Y, Horie M, Kawano H, Sudo $\mathrm{K}$, Asano M, Watanabe K et al. (2001): TAG-1-deficient mice have marked elevation of adenosine A1 receptors in the hippocampus. Biochem Biophys Res Commun 281 (1), 220-226. Cited on $\mathrm{p} 47$ and 84.

Fünfschilling U and Reichardt LF (2002): Cre-mediated recombination in rhombic lip derivatives. Genesis 33 (4), 160-169. Cited on $\mathrm{p} 22$.

Gallyas F (1979): Silver staining of myelin by means of physical development. Neurol Res 1 (2), 203-209. Cited on p 102.

Gao Z, Ure K, Ables JL, Lagace DC, Nave KA, Goebbels S, Eisch AJ and Hsieh J (2009): Neurod1 is essential for the survival and maturation of adult-born neurons. Nat Neurosci 12 (9), 1090-1092. Cited on p 85.

García-López P, García-Marín V and Freire M (2006): Three-dimensional reconstruction and quantitative study of a pyramidal cell of a Cajal histological preparation. J Neurosci 26 (44), 11249-11252. Cited on $\mathrm{p} 4$.

Gaspard N, Gaillard A and Vanderhaeghen P (2009): Making cortex in a dish: In vitro corticopoiesis from embryonic stem cells. Cell Cycle $\underline{8}$ (16), 2491-2496. Cited on p 83.

Gautier M, Flori L, Riebler A, Jaffrézic F, Laloé D, Gut I, Moazami-Goudarzi K and Foulley JL (2009): A whole genome Bayesian scan for adaptive genetic divergence in West African cattle. BMC Genomics 10, 550. Cited on p 92.

Genuardi M, Calvieri F, Tozzi C, Coslovi R and Neri G (1994): A new case of interstitial deletion of chromosome 3q, del(3q)(q13.12q21.3), with agenesis of the corpus callosum. Clin Dysmorphol 3 (4), 292-296. Cited on p 46.

Ghosh A and Shatz CJ (1993): A role for subplate neurons in the patterning of connections from thalamus to neocortex. Development 117 (3), 1031-47. Cited on p 39.

Goebbels S: Zelltyp-spezifische Expression der Rekombinase Cre im Nervensystem der Maus. Biol. Diss. Heidelberg 2002. Cited on p 18, 19, 28, 30, 38, 59, 60, 70, 88, and 129.

Goebbels S, Bode U, Pieper A, Funfschilling U, Schwab MH and Nave KA (2005): Cre/loxP-mediated inactivation of the bHLH transcription factor gene NeuroD/BETA2. Genesis 42 (4), 247-52. Cited on p 21, 22, 93, 95, and 129.

Goebbels S, Bormuth I, Bode U, Hermanson O, Schwab MH and Nave KA (2006): Genetic targeting of principal neurons in neocortex and hippocampus of NEX-Cre mice. Genesis 44 (12), 611-621. Cited on p 19, 21, 23, 27, 28, 30, 33, 38, 59, 60, 70, 86, 88, 93, 97, and 129.

Golgi C (1873): Sulla struttura della sostanza grigia del cervello. Gazzetta Medica Italiana Lombardia 33, 244-246. Cited on $\mathrm{p} 4$.

Gordon JW, Scangos GA, Plotkin DJ, Barbosa JA and Ruddle FH (1980): Genetic transformation of mouse embryos by microinjection of purified DNA. Proc Natl Acad Sci USA 77 (12), 7380-7384. Cited on p 4.

Goslin K, Schreyer DJ, Skene JH and Banker G (1988): Development of neuronal polarity: GAP-43 distinguishes axonal from dendritic growth cones. Nature 336 (6200), 672-674. Cited on $\mathrm{p} 46$. 
Grasselli G and Strata P (2013): Structural plasticity of climbing fibers and the growthassociated protein GAP-43. Front Neural Circuits $\underline{7}$, 25. Cited on p 91.

Grove EA, Tole S, Limon J, Yip L and Ragsdale CW (1998): The hem of the embryonic cerebral cortex is defined by the expression of multiple Wnt genes and is compromised in Gli3-deficient mice. Development 125 (12), 2315-2325. Cited on p 7.

Guerrini R and Parrini E (2010): Neuronal migration disorders. Neurobiol Dis 38 (2), 154-166. Cited on $\mathrm{p} 76$.

Gulisano M, Broccoli V, Pardini C and Boncinelli E (1996): Emx1 and Emx2 show different patterns of expression during proliferation and differentiation of the developing cerebral cortex in the mouse. Eur J Neurosci 8 (5), 1037-50. Cited on p 84.

Gummert M: Vergleichende Expressionsanalyse basischer Helix-Loop-Helix Proteine der NeuroD-Subfamilie im Zentralnervensystem der Maus. Biol. Diss. Göttingen 2003. Cited on p 18, 19, 20, 30, 55, and 74 .

Guo $Z$, Zhang $\mathrm{L}$, Wu $Z$, Chen $\mathrm{Y}$, Wang $\mathrm{F}$ and Chen $\mathrm{G}$ (2014): In vivo direct reprogramming of reactive glial cells into functional neurons after brain injury and in an Alzheimer's disease model. Cell Stem Cell 14 (2), 188-202. Cited on p 91.

Han MH, Jiao S, Jia JM, Chen Y, Chen CY, Gucek M, Markey SP and Li Z (2013): The novel caspase-3 substrate Gap43 is involved in AMPA receptor endocytosis and longterm depression. Mol Cell Proteomics 12 (12), 3719-3731. Cited on p 91.

Han W, Kwan KY, Shim S, Lam MMS, Shin Y, Xu X, Zhu Y, Li M and Sestan N (2011): TBR1 directly represses Fezf2 to control the laminar origin and development of the corticospinal tract. Proc Natl Acad Sci USA 108 (7), 3041-3046. Cited on p 37.

Hand R and Polleux F (2011): Neurogenin2 regulates the initial axon guidance of cortical pyramidal neurons projecting medially to the corpus callosum. Neural Dev 6, 30. Cited on $\mathrm{p} 74$.

Hand R, Bortone D, Mattar P, Nguyen L, Heng JIT, Guerrier S, Boutt E, Peters E, Barnes AP, Parras C et al. (2005): Phosphorylation of Neurogenin2 specifies the migration properties and the dendritic morphology of pyramidal neurons in the neocortex. Neuron 48 (1), 45-62. Cited on p 74.

Hannula-Jouppi K, Kaminen-Ahola N, Taipale M, Eklund R, Nopola-Hemmi J, Kääriäinen $\mathrm{H}$ and Kere $\mathrm{J}$ (2005): The axon guidance receptor gene ROBO1 is a candidate gene for developmental dyslexia. PLoS Genet $\underline{1}$ (4), e50. Cited on p 84.

Hanwell MD, Curtis DE, Lonie DC, Vandermeersch T, Zurek E and Hutchison GR (2012): Avogadro: an advanced semantic chemical editor, visualization, and analysis platform. J Cheminform 4 (1), 17. Cited on $\mathrm{p} 107$.

Hatanaka Y and Murakami F (2002): In vitro analysis of the origin, migratory behavior, and maturation of cortical pyramidal cells. J Comp Neurol 454 (1), 1-14. Cited on p 80.

Hatanaka Y and Yamauchi K (2013): Excitatory cortical neurons with multipolar shape establish neuronal polarity by forming a tangentially oriented axon in the intermediate zone. Cereb Cortex 23 (1), 105-113. Cited on p 78.

He Y, Wang J, Wang L, Chen ZJ, Yan C, Yang H, Tang H, Zhu C, Gong Q, Zang Y et al. (2009): Uncovering intrinsic modular organization of spontaneous brain activity in humans. PLoS ONE 4 (4), e5226. Cited on $\mathrm{p} 3$.

Hébert JM and Fishell G (2008): The genetics of early telencephalon patterning: some assembly required. Nat Rev Neurosci 9 (9), 678-685. Cited on p 7.

Herron P and Johnson JI (1987): Organization of intracortical and commissural connections in somatosensory cortical areas I and II in the raccoon. J Comp Neurol 257 (3), 359-371. Cited on $\mathrm{p} 13$. 
Heuer H, Christ S, Friedrichsen S, Brauer D, Winckler M, Bauer K and Raivich G (2003): Connective tissue growth factor: a novel marker of layer VII neurons in the rat cerebral cortex. Neuroscience 119 (1), 43-52. Cited on p 39.

Hevner RF, Shi L, Justice N, Hsueh Y, Sheng M, Smiga S, Bulfone A, Goffinet AM, Campagnoni AT and Rubenstein JL (2001): Tbr1 regulates differentiation of the preplate and layer 6. Neuron 29 (2), 353-66. Cited on p 36 and 84.

Hippenmeyer S, Vrieseling E, Sigrist M, Portmann T, Laengle C, Ladle DR and Arber S (2005): A developmental switch in the response of DRG neurons to ETS transcription factor signaling. PLoS Biol 3 (5), e159. Cited on p 52, 93, and 129.

Holahan MR, Honegger KS and Routtenberg A (2010): Ectopic growth of hippocampal mossy fibers in a mutated GAP-43 transgenic mouse with impaired spatial memory retention. Hippocampus 20 (1), 58-64. Cited on p 46.

Howell BW, Hawkes R, Soriano P and Cooper JA (1997): Neuronal position in the developing brain is regulated by mouse Disabled-1. Nature 389 (6652), 733-737. Cited on p 81.

Huang Z, Kawase-Koga Y, Zhang S, Visvader J, Toth M, Walsh CA and Sun T (2009): Transcription factor Lmo4 defines the shape of functional areas in developing cortices and regulates sensorimotor control. Dev Biol 327 (1), 132-142. Cited on p 41 and 55.

Ince-Dunn G, Hall BJ, Hu SC, Ripley B, Huganir RL, Olson JM, Tapscott SJ and Ghosh A (2006): Regulation of thalamocortical patterning and synaptic maturation by NeuroD2. Neuron 49 (5), 683-95. Cited on p 2, 23, 27, 38, 82, 85, and 141.

Innocenti GM and Vercelli A (2010): Dendritic Bundles, Minicolumns, Columns, and Cortical Output Units. Front Neuroanat $\underline{4}$. Cited on p 44.

Izant JG and McIntosh JR (1980): Microtubule-associated proteins: a monoclonal antibody to MAP2 binds to differentiated neurons. Proc Natl Acad Sci USA 77 (8), 47414745. Cited on $\mathrm{p} 37$.

Johnson MB, Wang PP, Atabay KD, Murphy EA, Doan RN, Hecht JL and Walsh CA (2015): Single-cell analysis reveals transcriptional heterogeneity of neural progenitors in human cortex. Nat Neurosci 18 (5), 637-646. Cited on p 74.

Jones L, López-Bendito G, Gruss P, Stoykova A and Molnár Z (2002): Pax6 is required for the normal development of the forebrain axonal connections. Development 129 (21), 5041-52. Cited on $\mathrm{p} 84$.

Joshi PS, Molyneaux BJ, Feng L, Xie X, Macklis JD and Gan L (2008): Bhlhb5 regulates the postmitotic acquisition of area identities in layers II-V of the developing neocortex. Neuron 60 (2), 258-272. Cited on p 17 and 82.

Jossin Y, Bar I, Ignatova N, Tissir F, De Rouvroit CL and Goffinet AM (2003): The reelin signaling pathway: some recent developments. Cereb Cortex 13 (6), 627-633. Cited on $\mathrm{p} 39$.

Kageyama R, Ohtsuka T, Hatakeyama J and Ohsawa R (2005): Roles of bHLH genes in neural stem cell differentiation. Exp Cell Res 306 (2), 343-348. Cited on p 74.

Kandel ER, Schwartz JH and Jessell TM: Principles of Neural Science. Mcgraw-Hill Professional 2000, 4th ed. Cited on $\mathrm{p} 6$.

Kandel ER, Markram H, Matthews PM, Yuste R and Koch C (2013): Neuroscience thinks big (and collaboratively). Nat Rev Neurosci 14 (9), 659-664. Cited on p 5 and 76.

Kaneko T, Cho R, Li Y, Nomura S and Mizuno N (2000): Predominant information transfer from layer III pyramidal neurons to corticospinal neurons. J Comp Neurol 423 (1), 5265. Cited on p 13.

Kawakami H, Maruyama H, Yasunami M, Ohkubo H, Hara H, Saida T, Nakanishi S and Nakamura S (1996): Cloning and expression of a rat brain basic helix-loop-helix factor. Biochem Biophys Res Commun 221 (1), 199-204. Cited on p 18. 
Keeling KB and Pavur RJ (2011): Statistical Accuracy of Spreadsheet Software. The American Statistician 65 (4), 265-273. Cited on p 106.

Kent G: Comparative Anatomy of the Vertebrates. McGraw-Hill Science/Engineering/Math 1954. Cited on p 6.

Kevanishvili ZS, Mosidze VM and Rizhinashvili RS (1969): The corpus callosum in interhemispheric transmission and bilateral synchronization of paroxysmal activity. Neurosci Behav Physiol 3 (2), 92-98. Cited on p 13.

Kim J, Choi M, Kim JR, Jin H, Kim VN and Cho KH (2012): The co-regulation mechanism of transcription factors in the human gene regulatory network. Nucleic Acids Res 40 (18), 8849-8861. Cited on p 21 and 25.

Kim MH, Gunnersen J, Augustine C and Tan SS (2002): Region-specific expression of the helix-loop-helix gene BETA3 in developing and adult brains. Mech Dev 114 (1-2), 125-128. Cited on $\mathrm{p} 88$.

Kim WY (2013): NeuroD Regulates Neuronal Migration. Mol Cells 35 (5), 444-449. Cited on $\mathrm{p} 87$.

Kim WY, Fritzsch B, Serls A, Bakel LA, Huang EJ, Reichardt LF, Barth DS and Lee JE (2001): NeuroD-null mice are deaf due to a severe loss of the inner ear sensory neurons during development. Development 128 (3), 417-426. Cited on p 86.

Knuth DE: Computers e type setting: a the texbook. Addison-Wesley, Reading 1986. Cited on p 108.

Koester SE and O'Leary DD (1993): Connectional distinction between callosal and subcortically projecting cortical neurons is determined prior to axon extension. Dev Biol 160 (1), 1-14. Cited on $\mathrm{p} 13$.

Kogan SC, Doherty M and Gitschier J (1987): An improved method for prenatal diagnosis of genetic diseases by analysis of amplified DNA sequences. Application to hemophilia A. N Engl J Med 317 (16), 985-990. Cited on p 96.

Kohm M: KOMA-Script: Eine Sammlung von Klassen und Paketen für LaTeX $2 e$. Lehmanns, Berlin 2014, auflage: 5., überarbeitete und erweiterte auflage ed. Cited on $\mathrm{p} 108$.

Koller BH, Hagemann LJ, Doetschman T, Hagaman JR, Huang S, Williams PJ, First NL, Maeda N and Smithies O (1989): Germ-line transmission of a planned alteration made in a hypoxanthine phosphoribosyltransferase gene by homologous recombination in embryonic stem cells. Proc Natl Acad Sci USA 86 (22), 8927-8931. Cited on p 5.

Kreibich TA, Chalasani SH and Raper JA (2004): The neurotransmitter glutamate reduces axonal responsiveness to multiple repellents through the activation of metabotropic glutamate receptor 1. J Neurosci 24 (32), 7085-7095. Cited on p 12.

Kume H, Maruyama K, Tomita T, Iwatsubo T, Saido TC and Obata K (1996): Molecular cloning of a novel basic helix-loop-helix protein from the rat brain. Biochem Biophys Res Commun 219 (2), 526-530. Cited on p 18 and 19.

Kuwabara T, Hsieh J, Muotri A, Yeo G, Warashina M, Lie DC, Moore L, Nakashima K, Asashima M and Gage FH (2009): Wnt-mediated activation of NeuroD1 and retroelements during adult neurogenesis. Nat Neurosci 12 (9), 1097-1105. Cited on p 85.

Kwan KY, Lam MMS, Krsnik Z, Kawasawa YI, Lefebvre V and Sestan N (2008): SOX5 postmitotically regulates migration, postmigratory differentiation, and projections of subplate and deep-layer neocortical neurons. Proc Natl Acad Sci USA 105 (41), 1602116026. Cited on $\mathrm{p} 42$.

Lai T, Jabaudon D, Molyneaux BJ, Azim E, Arlotta P, Menezes JRL and Macklis JD (2008): SOX5 controls the sequential generation of distinct corticofugal neuron subtypes. Neuron 57 (2), 232-47. Cited on p 42. 
Lalonde R and Strazielle C (2011): Brain regions and genes affecting limb-clasping responses. Brain Res Rev 67 (1-2), 252-259. Cited on p 31.

Lamport L: LATEX: a document preparation system : user's guide and reference manual. Addison-Wesley Pub. Co., Reading, Mass. 1994. Cited on p 108.

Lander ES, Linton LM, Birren B, Nusbaum C, Zody MC, Baldwin J, Devon K, Dewar $\mathrm{K}$, Doyle M, FitzHugh W et al. (2001): Initial sequencing and analysis of the human genome. Nature 409 (6822), 860-921. Cited on p 5.

Ledent V, Paquet O and Vervoort M (2002): Phylogenetic analysis of the human basic helix-loop-helix proteins. Genome Biol 3 (6), RESEARCH0030. Cited on p 14 and 15.

Lee JE, Hollenberg SM, Snider L, Turner DL, Lipnick N and Weintraub H (1995): Conversion of Xenopus ectoderm into neurons by NeuroD, a basic helix-loop-helix protein. Science 268 (5212), 836-844. Cited on p 17, 18, and 19.

Lee SK and Pfaff SL (2003): Synchronization of Neurogenesis and Motor Neuron Specification by Direct Coupling of bHLH and Homeodomain Transcription Factors. Neuron 38 (5), 731-745. Cited on $\mathrm{p} 16$.

Leone DP, Srinivasan K, Chen B, Alcamo E and McConnell SK (2008): The determination of projection neuron identity in the developing cerebral cortex. Curr Opin Neurobiol 18 (1), 28-35. Cited on p 41.

Leroy X (1990): The ZINC experiment, an economical implementation of the ML language. Technikal report 117, INRIA, Rocquencourt. Cited on p 107.

Lin CH, Stoeck J, Ravanpay AC, Guillemot F, Tapscott SJ and Olson JM (2004): Regulation of NeuroD2 expression in mouse brain. Dev Biol 265 (1), 234-45. Cited on p 19, 33, $60,70,86,87$, and 88 .

Lin CH, Hansen S, Wang Z, Storm DR, Tapscott SJ and Olson JM (2005): The dosage of the NeuroD2 transcription factor regulates amygdala development and emotional learning. Proc Natl Acad Sci U S A 102 (41), 14877-82. Cited on p 22, 23, 38, 85, and 87.

Lin CH, Tapscott SJ and Olson JM (2006): Congenital hypothyroidism (cretinism) in NeuroD2-deficient mice. Mol Cell Biol 26 (11), 4311-5. Cited on p 22, 43, and 87.

Lindwall C, Fothergill $\mathrm{T}$ and Richards LJ (2007): Commissure formation in the mammalian forebrain. Curr Opin Neurobiol 17 (1), 3-14. Cited on p 48, 49, and 83.

Lipnick S and Jacobson M: Developmental Neurobiology. Springer Science \& Business Media 2006. Cited on $\mathrm{p} 37$.

Liu M, Pleasure SJ, Collins AE, Noebels JL, Naya FJ, Tsai MJ and Lowenstein DH (2000): Loss of BETA2/NeuroD leads to malformation of the dentate gyrus and epilepsy. Proc Natl Acad Sci U S A 97 (2), 865-70. Cited on p 2, 21, 27, 32, 35, 60, 85, 86, and 141.

Lo CYZ, He Y and Lin CP (2011): Graph theoretical analysis of human brain structural networks. Rev Neurosci 22 (5), 551-563. Cited on $\mathrm{p} 3$.

Long H, Sabatier C, Ma L, Plump A, Yuan W, Ornitz DM, Tamada A, Murakami F, Goodman CS and Tessier-Lavigne M (2004): Conserved roles for Slit and Robo proteins in midline commissural axon guidance. Neuron 42 (2), 213-223. Cited on p 50.

Longo A, Guanga GP and Rose RB (2008): Crystal structure of E47-NeuroD1 /Beta2 bHLH domain-DNA complex: heterodimer selectivity and DNA recognition. Biochemistry 47 (1), 218-229. Cited on p 14 and 15.

López-Bendito G, Flames N, Ma L, Fouquet C, Di Meglio T, Chedotal A, Tessier-Lavigne $\mathrm{M}$ and Marin O (2007): Robo1 and Robo2 cooperate to control the guidance of major axonal tracts in the mammalian forebrain. J Neurosci 27 (13), 3395-3407. Cited on p 12, 83 , and 84 .

Ma Q, Kintner C and Anderson DJ (1996): Identification of Neurogenin, a vertebrate neuronal determination gene. Cell 87 (1), 43-52. Cited on p 17 and 74. 
Magdaleno S, Jensen P, Brumwell CL, Seal A, Lehman K, Asbury A, Cheung T, Cornelius T, Batten DM, Eden C et al. (2006): BGEM: an in situ hybridization database of gene expression in the embryonic and adult mouse nervous system. PLoS Biol $\underline{4}$ (4), e86. Cited on $\mathrm{p} 74$.

Maino B, Ciotti MT, Calissano P and Cavallaro S (2014): Transcriptional Analysis of Apoptotic Cerebellar Granule Neurons Following Rescue by Gastric Inhibitory Polypeptide. Int J Mol Sci 15 (4), 5596-5622. Cited on p 86.

Marin O (2013): Cellular and molecular mechanisms controlling the migration of neocortical interneurons. Eur J Neurosci 38 (1), 2019-2029. Cited on p 6.

Mattar P, Langevin LM, Markham K, Klenin N, Shivji S, Zinyk D and Schuurmans C (2008): Basic helix-loop-helix transcription factors cooperate to specify a cortical projection neuron identity. Mol Cell Biol 28 (5), 1456-69. Cited on p 20, 74, and 75.

Mattheisen M, Samuels JF, Wang Y, Greenberg BD, Fyer AJ, McCracken JT, Geller DA, Murphy DL, Knowles JA, Grados MA et al. (2014): Genome-wide association study in obsessive-compulsive disorder: results from the OCGAS. Mol Psychiatry . Cited on p 2 and 92 .

McCormick MB, Tamimi RM, Snider L, Asakura A, Bergstrom D and Tapscott SJ (1996): NeuroD2 and neuroD3: distinct expression patterns and transcriptional activation potentials within the neuroD gene family. Mol Cell Biol 16 (10), 5792-5800, pMC231580. Cited on $\mathrm{p} 19$.

McEvilly RJ, de Diaz MO, Schonemann MD, Hooshmand F and Rosenfeld MG (2002): Transcriptional regulation of cortical neuron migration by POU domain factors. Science 295 (5559), 1528-1532. Cited on $\mathrm{p} 40$.

McIlvain VA, Robertson DR, Maimone MM and McCasland JS (2003): Abnormal thalamocortical pathfinding and terminal arbors lead to enlarged barrels in neonatal GAP-43 heterozygous mice. J Comp Neurol 462 (2), 252-264. Cited on p 46.

McKenna WL, Betancourt J, Larkin KA, Abrams B, Guo C, Rubenstein JLR and Chen B (2011): Tbr1 and Fezf2 regulate alternate corticofugal neuronal identities during neocortical development. J Neurosci 31 (2), 549-564. Cited on p 37 and 64.

Meunier D, Lambiotte R and Bullmore ET (2010): Modular and hierarchically modular organization of brain networks. Front Neurosci $\underline{4}$, 200. Cited on $\mathrm{p} 3$.

Mikhailova O: The role of bHLH transcription factor NEX in neuronal differentiation and experience-dependent plasticity. Biol. Diss. Göttingen 2008. Cited on p 89 and 90.

Minichiello L and Klein R (1996): TrkB and TrkC neurotrophin receptors cooperate in promoting survival of hippocampal and cerebellar granule neurons. Genes Dev 10 (22), 2849-2858. Cited on p 86.

Miyata T, Maeda T and Lee JE (1999): NeuroD is required for differentiation of the granule cells in the cerebellum and hippocampus. Genes Dev 13 (13), 1647-52. Cited on p 2, 21, $27,35,60,85$, and 141.

Molin AM, Andrieux J, Koolen DA, Malan V, Carella M, Colleaux L, Cormier-Daire V, David A, de Leeuw N, Delobel B et al. (2012): A novel microdeletion syndrome at $3 q 13.31$ characterised by developmental delay, postnatal overgrowth, hypoplastic male genitals, and characteristic facial features. J Med Genet 49 (2), 104-109. Cited on p 46.

Molnár Z and Molnár E (2006): Calcium and NeuroD2 control the development of thalamocortical communication. Neuron 49 (5), 639-42. Cited on p 2 and 141.

Molnár Z and Pollen A (2014): How unique is the human neocortex? Development 141 (1), 11-16. Cited on $\mathrm{p} 77$.

Molnár Z, Garel S, López-Bendito G, Maness P and Price DJ (2012): Mechanisms controlling the guidance of thalamocortical axons through the embryonic forebrain. Eur J Neurosci 35 (10), 1573-1585. Cited on p 71. 
Molyneaux BJ, Arlotta P, Hirata T, Hibi M and Macklis JD (2005): Fezl is required for the birth and specification of corticospinal motor neurons. Neuron $\underline{47}$ (6), 817-831. Cited on $\mathrm{p} 42$.

Molyneaux BJ, Arlotta P, Menezes JRL and Macklis JD (2007): Neuronal subtype specification in the cerebral cortex. Nat Rev Neurosci 8 (6), 427-37. Cited on p 11 and 36.

Morin A, Urban J and Sliz P (2012): A quick guide to software licensing for the scientistprogrammer. PLoS Comput Biol 8 (7), e1002598. Cited on p 131 and 132.

Mullen RJ, Buck CR and Smith AM (1992): NeuN, a neuronal specific nuclear protein in vertebrates. Development 116 (1), 201-211. Cited on p 3 and 36.

Mullis K, Faloona F, Scharf S, Saiki R, Horn G and Erlich H (1986): Specific enzymatic amplification of DNA in vitro: the polymerase chain reaction. Cold Spring Harb Symp Guant Biol 51 Pt 1, 263-73. Cited on p 94.

Murdoch A, Jenkinson EJ, Johnson GD and Owen JJ (1990): Alkaline phosphatase-fast red, a new fluorescent label. Application in double labelling for cell cycle analysis. J Immunol Methods 132 (1), 45-49. Cited on p 104.

Murre C, Bain G, van Dijk MA, Engel I, Furnari BA, Massari ME, Matthews JR, Quong MW, Rivera RR and Stuiver MH (1994): Structure and function of helix-loop-helix proteins. Biochim Biophys Acta 1218 (2), 129-135. Cited on $\mathrm{p} 15$.

Nadarajah B, Brunstrom JE, Grutzendler J, Wong RO and Pearlman AL (2001): Two modes of radial migration in early development of the cerebral cortex. Nat Neurosci $\underline{4}$ (2), 143-150. Cited on $\mathrm{p} 80$.

Nagy A (2000): Cre recombinase: The universal reagent for genome tailoring. Genesis 26 (2), 99-109. Cited on p 25.

Navarrete K, Pedroso I, De Jong S, Stefansson H, Steinberg S, Stefansson K, Ophoff RA, Schalkwyk LC and Collier DA (2013): TCF4 (e2-2; ITF2): a schizophrenia-associated gene with pleiotropic effects on human disease. Am J Med Genet B Neuropsychiatr Genet 162B (1), 1-16. Cited on p 92.

Naya FJ, Stellrecht CM and Tsai MJ (1995): Tissue-specific regulation of the insulin gene by a novel basic helix-loop-helix transcription factor. Genes Dev 9 (8), 1009-1019. Cited on $\mathrm{p} 18$.

Naya FJ, Huang HP, Qiu Y, Mutoh H, DeMayo FJ, Leiter AB and Tsai MJ (1997): Diabetes, defective pancreatic morphogenesis, and abnormal enteroendocrine differentiation in BETA2/NeuroD-deficient mice. Genes Dev 11 (18), 2323-2334. Cited on p 19 and 21 .

Neher E and Sakmann B (1976): Single-channel currents recorded from membrane of denervated frog muscle fibres. Nature 260 (5554), 799-802. Cited on p 4.

Nieto M, Schuurmans C, Britz O and Guillemot F (2001): Neural bHLH genes control the neuronal versus glial fate decision in cortical progenitors. Neuron 29 (2), 401-413. Cited on $\mathrm{p} 74$.

Ninkovic J, Steiner-Mezzadri A, Jawerka M, Akinci U, Masserdotti G, Petricca S, Fischer $\mathrm{J}$, von Holst A, Beckers J, Lie CD et al. (2013): The BAF complex interacts with Pax6 in adult neural progenitors to establish a neurogenic cross-regulatory transcriptional network. Cell Stem Cell 13 (4), 403-418. Cited on p 79.

Noctor SC, Martínez-Cerdeño V, Ivic L and Kriegstein AR (2004): Cortical neurons arise in symmetric and asymmetric division zones and migrate through specific phases. Nat Neurosci $\underline{7}$ (2), 136-144. Cited on p 10, 76, 77, 78, and 80.

Noma T, Yoon YS and Nakazawa A (1999): Overexpression of NeuroD in PC12 cells alters morphology and enhances expression of the adenylate kinase isozyme 1 gene. Brain Res Mol Brain Res 67 (1), 53-63. Cited on p 19. 
North HA, Clifford MA and Donoghue MJ (2013): 'Til Eph do us part': intercellular signaling via Eph receptors and ephrin ligands guides cerebral cortical development from birth through maturation. Cereb Cortex 23 (8), 1765-1773. Cited on p 12.

Ohkubo Y, Chiang C and Rubenstein JLR (2002): Coordinate regulation and synergistic actions of BMP4, SHH and FGF8 in the rostral prosencephalon regulate morphogenesis of the telencephalic and optic vesicles. Neuroscience 111 (1), 1-17. Cited on p 7.

Ohtaka-Maruyama C, Hirai S, Miwa A, Heng JIT, Shitara H, Ishii R, Taya C, Kawano H, Kasai M, Nakajima K et al. (2013): RP58 regulates the multipolar-bipolar transition of newborn neurons in the developing cerebral cortex. Cell Rep 3 (2), 458-471. Cited on p 37.

Okhotin VE and Kalinichenko SG (2003): Subcortical white matter interstitial cells: their connections, neurochemical specialization, and role in the histogenesis of the cortex. Neurosci Behav Physiol 33 (2), 177-194. Cited on p 39.

O'Leary DDM, Chou SJ and Sahara S (2007a): Area patterning of the mammalian cortex. Neuron 56 (2), 252-269. Cited on $\mathrm{p} 8$ and 82.

O'Leary DDM, Chou SJ and Sahara S (2007b): Area patterning of the mammalian cortex. Neuron 56 (2), 252-269. Cited on p 39.

Olson JM, Asakura A, Snider L, Hawkes R, Strand A, Stoeck J, Hallahan A, Pritchard J and Tapscott SJ (2001): NeuroD2 is necessary for development and survival of central nervous system neurons. Dev Biol 234 (1), 174-87. Cited on p 22, 23, 27, 31, 33, 38, 60, 70, 85, 86, and 87.

Pakkenberg B, Pelvig D, Marner L, Bundgaard MJ, Gundersen HJG, Nyengaard JR and Regeur L (2003): Aging and the human neocortex. Exp Gerontol 38 (1-2), 95-99. Cited on $\mathrm{p} 3$.

Palani S and Sarkar CA (2009): Integrating extrinsic and intrinsic cues into a minimal model of lineage commitment for hematopoietic progenitors. PLoS Comput Biol $\underline{5}$ (9), e1000518. Cited on $\mathrm{p} 3$.

Pattabiraman K, Golonzhka O, Lindtner S, Nord AS, Taher L, Hoch R, Silberberg SN, Zhang D, Chen B, Zeng $\mathrm{H}$ et al. (2014): Transcriptional regulation of enhancers active in protodomains of the developing cerebral cortex. Neuron 82 (5), 989-1003. Cited on p 8.

Pattyn A, Guillemot F and Brunet JF (2006): Delays in neuronal differentiation in Mash1/Ascl1 mutants. Dev Biol 295 (1), 67-75. Cited on p 33.

Pérez-Santiago J, Diez-Alarcia R, Callado LF, Zhang JX, Chana G, White CH, Glatt SJ, Tsuang MT, Everall IP, Meana JJ et al. (2012): A combined analysis of microarray gene expression studies of the human prefrontal cortex identifies genes implicated in schizophrenia. Journal of Psychiatric Research 46 (11), 1464-1474. Cited on p 2 and 92.

Peters A and Sethares C (1996): Myelinated axons and the pyramidal cell modules in monkey primary visual cortex. J Comp Neurol 365 (2), 232-255. Cited on p 44.

Peyton M, Stellrecht CM, Naya FJ, Huang HP, Samora PJ and Tsai MJ (1996): BETA3, a novel helix-loop-helix protein, can act as a negative regulator of BETA2 and MyoDresponsive genes. Mol Cell Biol 16 (2), 626-633. Cited on p 20.

Picker A, Scholpp S, Böhli H, Takeda H and Brand M (2002): A novel positive transcriptional feedback loop in midbrain-hindbrain boundary development is revealed through analysis of the zebrafish pax2.1 promoter in transgenic lines. Development 129 (13), 3227-3239. Cited on $\mathrm{p} 8$.

Pierce BC and Vouillon J (2004): What's in Unison? A Formal Specification and Reference Implementation of a File Synchronizer. Tech. Rep. MS-CIS-03-36, Dept. of Computer and Information Science, University of Pennsylvania. Cited on p 106.

Pleasure SJ, Collins AE and Lowenstein DH (2000): Unique Expression Patterns of Cell Fate Molecules Delineate Sequential Stages of Dentate Gyrus Development. J Neurosci 20 (16), 6095-6105. Cited on p 17, 74, and 75. 
Pollerberg GE, Thelen K, Theiss MO and Hochlehnert BC (2013): The role of cell adhesion molecules for navigating axons: density matters. Mech Dev 130 (6-8), 359-372. Cited on $\mathrm{p} 47$.

Polleux F, Morrow T and Ghosh A (2000): Semaphorin $3 a$ is a chemoattractant for cortical apical dendrites. Nature 404 (6778), 567-73. Cited on p 12.

Probst M (1901): Ueber den Bau des vollständig balkenlosen Gross-hirnes sowie über Mikrogyrie und Heterotopie der grauen Substanz. Archiv f Psychiatrie 34 (3), 709-786. Cited on $\mathrm{p} 49$.

R Development Core Team: R: A Language and Environment for Statistical Computing. R Foundation for Statistical Computing, Vienna, Austria 2008, iSBN 3-900051-07-0. Cited on $\mathrm{p} 106$.

Rajagopalan S, Nicolas E, Vivancos V, Berger J and Dickson BJ (2000): Crossing the midline: roles and regulation of Robo receptors. Neuron 28 (3), 767-777. Cited on p 84.

Ravanpay AC, Hansen SJ and Olson JM (2010): Transcriptional inhibition of REST by NeuroD2 during neuronal differentiation. Mol Cell Neurosci 44 (2), 178-189. Cited on p 91.

Reznikov KY: Cell Proliferation and Cytogenesis in the Mouse Hippocampus. Springer 1991. Cited on $\mathrm{p} 66$.

Richards LJ, Plachez C and Ren T (2004): Mechanisms regulating the development of the corpus callosum and its agenesis in mouse and human. Clin Genet 66 (4), 276-89. Cited on $\mathrm{p} 45$.

Richetin K, Leclerc C, Toni N, Gallopin T, Pech S, Roybon L and Rampon C (2015): Genetic manipulation of adult-born hippocampal neurons rescues memory in a mouse model of Alzheimer's disease. Brain 138 (Pt 2), 440-455. Cited on p 91.

Rolls ET (2013): The mechanisms for pattern completion and pattern separation in the hippocampus. Front Syst Neurosci 7, 74. Cited on p 59.

Rossant $J$ and McMahon A (1999): “Cre"-ating mouse mutants-a meeting review on conditional mouse genetics. Genes Dev 13 (2), 142-145. Cited on p 25.

Routtenberg A, Cantallops I, Zaffuto S, Serrano P and Namgung U (2000): Enhanced learning after genetic overexpression of a brain growth protein. Proc Natl Acad Sci USA 97 (13), 7657-7662. Cited on p 46.

Rowitch DH, Lu QR, Kessaris N and Richardson WD (2002): An 'oligarchy' rules neural development. Trends Neurosci 25 (8), 417-422. Cited on p 74.

Roztocil T, Matter-Sadzinski L, Alliod C, Ballivet M and Matter JM (1997): NeuroM, a neural helix-loop-helix transcription factor, defines a new transition stage in neurogenesis. Development 124 (17), 3263-72. Cited on p 17, 20, and 74.

Rubenstein JLR (2011): Annual Research Review: Development of the cerebral cortex: implications for neurodevelopmental disorders. J Child Psychol Psychiatry 52 (4), 339355. Cited on $\mathrm{p} 4$.

Rubenstein JLR and Rakic P: Patterning and Cell Type Specification in the Developing CNS and PNS: Comprehensive Developmental Neuroscience. Academic Press 2013. Cited on $\mathrm{p} 8$.

Rubin RD, Watson PD, Duff MC and Cohen NJ (2014): The role of the hippocampus in flexible cognition and social behavior. Front Hum Neurosci $\underline{8}$. Cited on $\mathrm{p} 59$.

Rudnicki MA, Schnegelsberg PN, Stead RH, Braun T, Arnold HH and Jaenisch R (1993): MyoD or Myf-5 is required for the formation of skeletal muscle. Cell 75 (7), 1351-1359. Cited on $\mathrm{p} 16$.

Ruediger T and Bolz J (2007): Neurotransmitters and the development of neuronal circuits. Adv Exp Med Biol 621, 104-115. Cited on p 12. 
Saba R, Johnson JE and Saito T (2005): Commissural neuron identity is specified by a homeodomain protein, Mbh1, that is directly downstream of Math1. Development 132 (9), 2147-2155. Cited on p 16.

Sairanen M, O'Leary OF, Knuuttila JE and Castrén E (2007): Chronic antidepressant treatment selectively increases expression of plasticity-related proteins in the hippocampus and medial prefrontal cortex of the rat. Neuroscience 144 (1), 368-374. Cited on p 91.

Saito T (2006): In vivo electroporation in the embryonic mouse central nervous system. Nat Protocols 1 (3), 1552-1558. Cited on p 99.

Sakakibara A and Hatanaka Y (2015): Neuronal polarization in the developing cerebral cortex. Front Neurosci 9, 116. Cited on p 11 and 78.

Sambrook J and Russell DW: Molecular Cloning: A Laboratory Manual, 3 Vol. Cold Spring Harbor Laboratory 2000, 0003rd ed. Cited on p 94 and 95.

Sansom SN, Griffiths DS, Faedo A, Kleinjan DJ, Ruan Y, Smith J, van Heyningen V, Rubenstein JL and Livesey FJ (2009): The level of the transcription factor Pax6 is essential for controlling the balance between neural stem cell self-renewal and neurogenesis. PLoS Genet $\underline{5}$ (6), e1000511. Cited on p 79.

Sarnat HB, Nochlin D and Born DE (1998): Neuronal nuclear antigen (NeuN): a marker of neuronal maturation in early human fetal nervous system. Brain Dev 20 (2), 88-94. Cited on $\mathrm{p} 3$ and 36.

Satoh Ji, Yamamoto Y, Asahina N, Kitano S and Kino Y (2014): RNA-Seq Data Mining: Downregulation of NeuroD6 Serves as a Possible Biomarker for Alzheimer's Disease Brains. Dis Markers 2014. Cited on p 2 and 92.

Scheetz D: The Debian Linux User's Guide. Linux Press, Penngrove 1998. Cited on p 106.

Schellenberg GD, Dawson G, Sung YJ, Estes A, Munson J, Rosenthal E, Rothstein J, Flodman P, Smith M, Coon H et al. (2006): Evidence for multiple loci from a genome scan of autism kindreds. Mol Psychiatry 11 (11), 1049-1060, 979. Cited on p 46.

Schlaggar BL and O'Leary DD (1991): Potential of visual cortex to develop an array of functional units unique to somatosensory cortex. Science 252 (5012), 1556-1560. Cited on $\mathrm{p} 81$.

Schneider CA, Rasband WS and Eliceiri KW (2012): NIH Image to ImageJ: 25 years of image analysis. Nat Methods $\underline{9}$ (7), 671-675. Cited on p 107.

Schuurmans C, Armant O, Nieto M, Stenman JM, Britz O, Klenin N, Brown C, Langevin LM, Seibt J, Tang $\mathrm{H}$ et al. (2004): Sequential phases of cortical specification involve Neurogenin-dependent and -independent pathways. EMBO J 23 (14), 2892-2902. Cited on $\mathrm{p} 74$ and 79.

Schwab MH, Druffel-Augustin S, Gass P, Jung M, Klugmann M, Bartholomae A, Rossner MJ and Nave KA (1998): Neuronal basic helix-loop-helix proteins (NEX, NeuroD, NDRF): spatiotemporal expression and targeted disruption of the NEX gene in transgenic mice. J Neurosci 18 (4), 1408-18. Cited on p 19, 23, 24, 27, 28, 30, 60, 86, and 89.

Schwab MH, Bartholomae A, Heimrich B, Feldmeyer D, Druffel-Augustin S, Goebbels S, Naya FJ, Zhao S, Frotscher M, Tsai MJ et al. (2000): Neuronal basic helix-loop-helix proteins (NEX and BETA2/NeuroD) regulate terminal granule cell differentiation in the hippocampus. J Neurosci 20 (10), 3714-24. Cited on p 2, 17, 23, 25, 27, 28, 30, 36, 60, 85, and 86 .

Sepp M, Pruunsild P and Timmusk T (2012): Pitt-Hopkins syndrome-associated mutations in TCF4 lead to variable impairment of the transcription factor function ranging from hypomorphic to dominant-negative effects. Hum Mol Genet 21 (13), 2873-2888. Cited on $\mathrm{p} 92$.

Serafini T, Colamarino SA, Leonardo ED, Wang H, Beddington R, Skarnes WC and Tessier-Lavigne M (1996): Netrin-1 is required for commissural axon guidance in the developing vertebrate nervous system. Cell 87 (6), 1001-14. Cited on p 83. 
Shen Y, Mani S, Donovan SL, Schwob JE and Meiri KF (2002): Growth-associated protein-43 is required for commissural axon guidance in the developing vertebrate nervous system. J Neurosci 22 (1), 239-247. Cited on p 46 and 83.

Shimizu C, Akazawa C, Nakanishi S and Kageyama R (1995): MATH-2, a mammalian helix-loop-helix factor structurally related to the product of Drosophila proneural gene atonal, is specifically expressed in the nervous system. Eur J Biochem 229 (1), 239248. Cited on p 19, 28, and 59.

Shinozaki K, Miyagi T, Yoshida M, Miyata T, Ogawa M, Aizawa S and Suda Y (2002): Absence of Cajal-Retzius cells and subplate neurons associated with defects of tangential cell migration from ganglionic eminence in Emx1/2 double mutant cerebral cortex. Development 129 (14), 3479-92. Cited on p 84.

Simpson EM, Linder CC, Sargent EE, Davisson MT, Mobraaten LE and Sharp JJ (1997): Genetic variation among 129 substrains and its importance for targeted mutagenesis in mice. Nat Genet 16 (1), 19-27. Cited on p 21.

Singer VL, Lawlor TE and Yue S (1999): Comparison of SYBR Green I nucleic acid gel stain mutagenicity and ethidium bromide mutagenicity in the Salmonella/mammalian microsome reverse mutation assay (Ames test). Mutat Res 439 (1), 37-47. Cited on p 95.

Skinner MK, Rawls A, Wilson-Rawls J and Roalson EH (2010): Basic Helix-LoopHelix Transcription Factor Gene Family Phylogenetics and Nomenclature. Differentiation 80 (1), 1-8. Cited on $\mathrm{p} 14$.

Smith DH (2009): Stretch growth of integrated axon tracts: Extremes and exploitations. Prog Neurobiol 89 (3), 231-239. Cited on p 11.

Sohur US, Padmanabhan HK, Kotchetkov IS, Menezes JRL and Macklis JD (2014): Anatomic and molecular development of corticostriatal projection neurons in mice. Cereb Cortex 24 (2), 293-303. Cited on p 14.

Soosaar A, Chiaramello A, Zuber MX and Neuman T (1994): Expression of basic-helixloop-helix transcription factor ME2 during brain development and in the regions of neuronal plasticity in the adult brain. Brain Res Mol Brain Res 25 (1-2), 176-180. Cited on $\mathrm{p} 89$.

Soriano P (1999): Generalized lacZ expression with the ROSA26 Cre reporter strain. Nat Genet 21 (1), 70-71. Cited on p 29, 93, and 129.

Squire LR (1992): Memory and the hippocampus: a synthesis from findings with rats, monkeys, and humans. Psychol Rev 99 (2), 195-231. Cited on p 59.

Srivatsa S, Parthasarathy S, Britanova O, Bormuth I, Donahoo AL, Ackerman SL, Richards LJ and Tarabykin V (2014): Unc5c and DCC act downstream of Ctip2 and Satb2 and contribute to corpus callosum formation. Nat Commun $\underline{5}$, 3708. Cited on $\mathrm{p} 12$ and 84 .

Strittmatter SM (2000): Dendrites go up, axons go down. Nature 404 (6778), 557, 559. Cited on $\mathrm{p} 12$.

Sugitani Y, Nakai S, Minowa O, Nishi M, Jishage KI, Kawano H, Mori K, Ogawa M and Noda T (2002): Brn-1 and Brn-2 share crucial roles in the production and positioning of mouse neocortical neurons. Genes Dev 16 (14), 1760-1765. Cited on p 40.

Sun T, Patoine C, Abu-Khalil A, Visvader J, Sum E, Cherry TJ, Orkin SH, Geschwind DH and Walsh CA (2005): Early asymmetry of gene transcription in embryonic human left and right cerebral cortex. Science 308 (5729), 1794-1798. Cited on p 55 and 65.

Surget A, Wang Y, Leman S, Ibarguen-Vargas Y, Edgar N, Griebel G, Belzung C and Sibille E (2009): Corticolimbic transcriptome changes are state-dependent and regionspecific in a rodent model of depression and of antidepressant reversal. Neuropsychopharmacology 34 (6), 1363-1380. Cited on p 91. 
Tabata H, Hachiya T, Nagata KI, Sakakibara Y and Nakajima K (2013): Screening for candidate genes involved in the production of mouse subventricular zone proliferative cells and an estimation of their changes in evolutionary pressure during primate evolution. Front Neuroanat 7, 24. Cited on p 75.

Taelman V, Opdecamp K, Avalosse B, Ryan K and Bellefroid EJ (2001): Xath2, a bHLH gene expressed during a late transition stage of neurogenesis in the forebrain of Xenopus embryos. Mech Dev 101 (1-2), 199-202. Cited on p 28.

Takahashi H and Liu FC (2006): Genetic patterning of the mammalian telencephalon by morphogenetic molecules and transcription factors. Birth Defects Res C Embryo Today $\underline{78}$ (3), 256-266. Cited on p 7.

Takahashi K, Liu FC, Hirokawa K and Takahashi H (2008): Expression of Foxp4 in the developing and adult rat forebrain. J Neurosci Res 86 (14), 3106-3116. Cited on p 42 and 65 .

Takiguchi-Hayashi K, Sekiguchi M, Ashigaki S, Takamatsu M, Hasegawa H, SuzukiMigishima R, Yokoyama M, Nakanishi S and Tanabe Y (2004): Generation of reelinpositive marginal zone cells from the caudomedial wall of telencephalic vesicles. $\mathrm{J}$ Neurosci 24 (9), 2286-2295. Cited on p 10.

Talikka M, Perez SE and Zimmerman K (2002): Distinct patterns of downstream target activation are specified by the helix-loop-helix domain of proneural basic helix-loop-helix transcription factors. Dev Biol 247 (1), 137-148. Cited on p 74.

Thévenaz P, Ruttimann UE and Unser M (1998): A pyramid approach to subpixel registration based on intensity. IEEE Trans Image Process 7 (1), 27-41. Cited on p 107.

Thivierge JP and Marcus GF (2007): The topographic brain: from neural connectivity to cognition. Trends Neurosci 30 (6), 251-259. Cited on p 3.

Thomas L: The Lives of a Cell. Viking Press 1974. Cited on $\mathrm{p} 27$.

Threadgill DW, Yee D, Matin A, Nadeau JH and Magnuson T (1997): Genealogy of the 129 inbred strains: 129/SvJ is a contaminated inbred strain. Mamm Genome $\underline{8}$ (6), 390-393. Cited on $\mathrm{p} 32$.

Tissir F and Goffinet AM (2003): Reelin and brain development. Nat Rev Neurosci 4 (6), 496-505. Cited on $\mathrm{p} 38$.

Tomita K, Moriyoshi K, Nakanishi S, Guillemot F and Kageyama R (2000): Mammalian achaete-scute and atonal homologs regulate neuronal versus glial fate determination in the central nervous system. EMBO J 19 (20), 5460-5472. Cited on p 17.

Trikalinos TA, Karvouni A, Zintzaras E, Ylisaukko-oja T, Peltonen L, Järvelä I and Ioannidis JPA (2006): A heterogeneity-based genome search meta-analysis for autismspectrum disorders. Mol Psychiatry 11 (1), 29-36. Cited on p 46.

Trommsdorff M, Gotthardt M, Hiesberger T, Shelton J, Stockinger W, Nimpf J, Hammer RE, Richardson JA and Herz J (1999): Reeler/Disabled-like disruption of neuronal migration in knockout mice lacking the VLDL receptor and ApoE receptor 2. Cell 97 (6), 689-701. Cited on $\mathrm{p} 81$.

Uittenbogaard M and Chiaramello A (2002): Constitutive overexpression of the basic helix-loop-helix Nex1/MATH-2 transcription factor promotes neuronal differentiation of PC12 cells and neurite regeneration. J Neurosci Res 67 (2), 235-245. Cited on p 46.

Uittenbogaard M and Chiaramello A (2005): The basic helix-loop-helix transcription factor Nex-1/Math-2 promotes neuronal survival of PC12 cells by modulating the dynamic expression of anti-apoptotic and cell cycle regulators. Journal of Neurochemistry 92 (3), 585-596. Cited on $\mathrm{p} 86$.

Uittenbogaard M, Martinka DL and Chiaramello A (2003): The basic helix-loop-helix differentiation factor Nex1/MATH-2 functions as a key activator of the GAP-43 gene. $\mathrm{J}$ Neurochem 84 (4), 678-688. Cited on p 46. 
Uittenbogaard M, Baxter KK and Chiaramello A (2009): Cloning and characterization of the 5'UTR of the rat anti-apoptotic Bcl-w gene. Biochemical and Biophysical Research Communications 389 (4), 657-662. Cited on p 86.

Unni DK, Piper M, Moldrich RX, Gobius I, Liu S, Fothergill T, Donahoo ALS, Baisden JM, Cooper HM and Richards LJ (2012): Multiple Slits regulate the development of midline glial populations and the corpus callosum. Developmental Biology 365 (1), 3649. Cited on $\mathrm{p} 12$ and 84 .

Venter JC, Adams MD, Myers EW, Li PW, Mural RJ, Sutton GG, Smith HO, Yandell M, Evans CA, Holt RA et al. (2001): The sequence of the human genome. Science 291 (5507), 1304-1351. Cited on p 5.

Villar-Cerviño V, Molano-Mazón M, Catchpole T, Valdeolmillos M, Henkemeyer M, Martínez LM, Borrell V and Marín O (2013): Contact repulsion controls the dispersion and final distribution of Cajal-Retzius cells. Neuron 77 (3), 457-471. Cited on p 37.

Visel A, Taher L, Girgis H, May D, Golonzhka O, Hoch RV, McKinsey GL, Pattabiraman K, Silberberg SN, Blow MJ et al. (2013): A high-resolution enhancer atlas of the developing telencephalon. Cell 152 (4), 895-908. Cited on p 8 and 75.

Vitriol EA and Zheng JQ (2012): Growth cone travel in space and time: the cellular ensemble of cytoskeleton, adhesion, and membrane. Neuron 73 (6), 1068-1081. Cited on p 70.

Voronova A and Baltimore D (1990): Mutations that disrupt DNA binding and dimer formation in the E47 helix-loop-helix protein map to distinct domains. Proc Natl Acad Sci USA 87 (12), 4722-4726. Cited on p 15.

Walantus W, Castaneda D, Elias L and Kriegstein A (2007): In utero intraventricular injection and electroporation of E15 mouse embryos. J Vis Exp (6), 239. Cited on p 99.

Wang Y, Geer LY, Chappey C, Kans JA and Bryant SH (2000): Cn3d: sequence and structure views for Entrez. Trends Biochem Sci 25 (6), 300-302. Cited on p 107.

Warburton EC, Barker GRI and Brown MW (2013): Investigations into the involvement of NMDA mechanisms in recognition memory. Neuropharmacology 74, 41-47. Cited on p 54.

Waterston RH, Lindblad-Toh K, Birney E, Rogers J, Abril JF, Agarwal P, Agarwala R, Ainscough R, Alexandersson M, An P et al. (2002): Initial sequencing and comparative analysis of the mouse genome. Nature 420 (6915), 520-562. Cited on p 5.

Weller RE, Sur M and Kaas JH (1987): Callosal and ipsilateral cortical connections of the body surface representations in SI and SII of tree shrews. Somatosens Res $\underline{5}$ (2), 107-133. Cited on $\mathrm{p} 13$.

Wilke SA, Hall BJ, Antonios JK, Denardo LA, Otto S, Yuan B, Chen F, Robbins EM, Tiglio K, Williams ME et al. (2012): NeuroD2 regulates the development of hippocampal mossy fiber synapses. Neural Dev $\underline{7}, 9$. Cited on $\mathrm{p} 2$.

Williams SE, Mason CA and Herrera E (2004): The optic chiasm as a midline choice point. Curr Opin Neurobiol 14 (1), 51-60. Cited on p 84.

Wojcik SM, Rhee JS, Herzog E, Sigler A, Jahn R, Takamori S, Brose N and Rosenmund C (2004): An essential role for vesicular glutamate transporter 1 (VGLUT1) in postnatal development and control of quantal size. Proc Natl Acad Sci USA 101 (18), 7158-7163. Cited on p 54 .

Wong-Riley MT and Welt C (1980): Histochemical changes in cytochrome oxidase of cortical barrels after vibrissal removal in neonatal and adult mice. Proc Natl Acad Sci USA 77 (4), 2333-2337. Cited on p 54.

Wu CS, Ballester Rosado CJ and Lu HC (2011): What can we get from 'barrels': the rodent barrel cortex as a model for studying the establishment of neural circuits. Eur $\mathrm{J}$ Neurosci 34 (10), 1663-1676. Cited on p 2. 
Wu SX, Goebbels S, Nakamura K, Nakamura K, Kometani K, Minato N, Kaneko T, Nave KA and Tamamaki N (2005): Pyramidal neurons of upper cortical layers generated by NEX-positive progenitor cells in the subventricular zone. Proc Natl Acad Sci U S A 102 (47), 17172-7. Cited on p 29.

Yamada M, Shida Y, Takahashi K, Tanioka T, Nakano Y, Tobe T and Yamada M (2008): Prg1 is regulated by the basic helix-loop-helix transcription factor Math2. Journal of Neurochemistry 106 (6), 2375-2384. Cited on p 92.

Yasunami M, Suzuki K, Maruyama H, Kawakami H, Nagai Y, Hagiwara M and Ohkubo H (1996): Molecular cloning and characterization of a cDNA encoding a novel basic helix-loop-helix protein structurally related to Neuro-D/BHF1. Biochem Biophys Res Commun 220 (3), 754-8. Cited on p 18 and 19.

Zaccaria KJ, Lagace DC, Eisch AJ and McCasland JS (2010): Resistance to change and vulnerability to stress: autistic-like features of GAP43-deficient mice. Genes Brain Behav 9 (8), 985-996. Cited on p 46. 


\section{Appendix}




\section{Acknowledgments}

\section{Personal Note}

I am very thankful for patient faith and unconditional support to my parents and family, Prof. Walter Paulus, Prof. Klaus-Armin Nave, Prof. Victor Tarabykin, and Prof. Mikael Simons. Thank you for all the help and scientific advice to Ajit, Alex, Annette, Bianka, Birgit, Carolin, Celia, Chis, Corinna, Daniel, Eva, Frank, Gabriele, Georg, Gudrun, Hajo, Hauke, Knuth, Kuo, Magda, Maike, Manu, Markus, Moritz, Olga, Olli, Rainer, Rolf, Sandra, Srini, Stefan, Steffi, Sven, Swathi, Tomoko and Ulli (lab mates, staff and friends in alphabetical order).

\section{Funding}

This work was funded by the Max-Planck-Society (MPG), the German Research Foundation (DFG), the University of Göttingen, and the German NeuroCure Cluster of Excellence.

\section{Transgenic Mice}

- Neurod6-Cre mice ${ }^{1}$ (sect 1.5.3; sect 2.2) and Neurod1-Flox mice ${ }^{2}$ (sect 1.5.1) were generated by Sandra Göbbels, Department of Neurogenetics, MaxPlanck-Institute of Experimental Medicine, Göttingen, Germany.

- Neurod2-Null mice ${ }^{3}$ (sect 1.5.2; sect 2.3; sect 2.5 ) were generated by Tomoko Yonemasu, Department of Neurogenetics, Max-Planck-Institute of Experimental Medicine, Göttingen, Germany.

- Rosa26 based Cre-reporter mice ${ }^{4}$ (sect 2.1) were provided by Philippe Soriano, Mount Sinai School of Medicine, New York, USA.

- Axonal Cre-reporter mice ${ }^{5}$ (sect 2.4.4.6) were provided by Silvia Arber, University of Basel, Switzerland.

\footnotetext{
${ }^{1}$ Goebbels 2002; Goebbels et al. 2006 (NEX-Cre)

${ }^{2}$ Goebbels et al. 2005 (Neurod1-Flox)

${ }^{3}$ Bormuth et al. 2013

${ }^{4}$ Soriano 1999: A LoxP-Neo-polyA-LoxP-LacZ-polyA genetrap cassette was inserted into the endogenous Rosa26 locus.

${ }^{5}$ Hippenmeyer et al. 2005: A LoxP-STOP-LoxP-mGFP-IRES-nLacZ-polyA targeting cassette was inserted into Exon 2 of the endogenous Mapt locus.
} 


\section{Collaborations}

- ISH (fig 8; fig 9; fig 15a-b; fig 32a-b) was initially performed together with Maike Gummert, Department of Neurogenetics, Max-Planck-Institute of Experimental Medicine, Göttingen, Germany.

- Electrophysiology (fig 30d-h) and iontophoresis (fig 25a, c) were performed together with Ming Zhang, Department of Neurophysiology, University of Göttingen (now Laboratory of Molecular Psychiatry, Department of Psychiatry, University of Münster), Germany.

\section{Antibodies}

- The Robol antibody (fig 28a) was provided by Fujio Murakami, Osaka University, Japan.

- The Lmo4 antibody (fig 22b; fig 31a, b) was provided by Jane E. Visvader, Walter and Eliza Hall Institute of Medical Research, Parkville, Australia.

- The Satb2 antibody ${ }^{1}$ (fig 22c; fig 23c; fig 37c) was provided by Victor Tarabykin, Charité, Berlin, Germany.

- Antibodies against Cntn2 (4D7Tag1 by M. Yamamoto; fig 26a, b) and L1cam (ASCS4/L1 by P. H. Patterson; fig 26a, b; fig 41b) were obtained from the Developmental Studies Hybridoma Bank ${ }^{2}$ developed under the auspices of the NICHD and maintained by The University of Iowa, Department of Biology, Iowa City, IA 52242.

\footnotetext{
${ }^{1}$ Britanova et al. 2008

${ }^{2}$ Developmental Studies Hybridoma Bank (DSHB) at the University of Iowa: http://dshb.biology. uiowa.edu
} 


\section{Abbreviations}

Gene symbols mostly follow the standard nomenclature of the mouse genome informatics database (MGI). Exceptions were made where the official naming is rarely used in the current literature of the field. Those cases are indicated by additionally providing the MGI symbol in underlined text. For better readability and to avoid conflicts with other abbreviations, protein symbols were mostly not converted to all upper-case.

AC anterior commissure

AGPL GNU affero general public license ${ }^{1}$ AL Apache license ${ }^{2}$

AMPA $\alpha$-amino-3-hydroxy-5-methyl-4isoxazolepropionic acid (glutamate receptor agonist)

AP alkaline phosphatase

Apoer2 apolipoprotein receptor related 8 $=\operatorname{Lrp8}$ gene id: 16975

BDNF brain derived neurotrophic factor (peptide of gene id: 12064)

bHLH basic helix-loop-helix

Bhlhe22 bHLH family member e22 = Bhlhb5 gene id: 59058

Blbp brain lipid binding protein gene id: 12140

BMP bone morphogenetic protein (gene/protein family)

bp base pair (also base pairs)

BrdU bromodeoxyuridine

Brn1 brain 1 = Pou3f3 gene id: 18993

Brn2 brain 2 = Pou3f2 gene id: 18992

BSA bovine serum albumin

BSD Berkeley software distribution license ${ }^{3}$

CA cornu ammonis

Calb2 calbindin $2=$ calretinin gene id: 12308

CAM cell adhesion molecule

CB cerebellum

CC corpus callosum

cDNA complementary DNA

Ci cingulate cortex

Cnp 2',3'-cyclic nucleotide 3' phosphodiesterase

CNS central nervous system

Cntn1 contactin 1 CAM $=$ F3cam gene id: 12805
Cntn2 contactin $2 C A M=$ Tag- 1 gene id: 21367

CO cytochrome oxidase

CP cortical plate

CPd cerebral peduncle

CR coronal radiation

Cre Cre recombinase

cRNA complementary RNA

CST corticospinal tract

Ctgf connective tissue growth factor $=$ Fisp gene id: 14219

Ctip2 COUP-TF interacting 2 $=$ Bcl1 $1 \mathrm{~b}$ gene id: 58208

Ctnnb1 $\beta$-Catenin (Cadherin associated protein $\beta 1$ ) gene id: 12387

ctrl control

CTT corticothalamic tract

Ctx cortex

Cux1 cut-like homeobox 1 gene id: 13047

d dorsal

DAB diaminobenzidine

Dab1 disabled homolog 1 $=$ scrambler gene id: 13131

DAPI 4',6-diamidino-2-phenylindole

Dcc deleted in colorectal carcinoma gene id: 13176

DEPC diethyl pyrocarbonate

DG dentate gyrus

DIG digoxigenin

DKO double-deficient mice ('double knockout')

DMSO dimethyl sulfoxide

DNA deoxyribonucleic acid

dNTP dATP + dCTP + dGTP + dTTP

DRG dorsal root ganglion

dTh dorsal thalamus

E embryonic day

E-box enhancer box (DNA motive)

EC external capsule

EDTA ethylene diamine tetraacetic acid

eg for example (exempli gratia, latin)

EGF epidermal growth factor (gene/protein family)

EGTA ethylene glycol tetraacetic acid

Emx1 empty spiracles homolog 1 gene id: 13796

Emx2 empty spiracles homolog 2 gene id: 13797

\footnotetext{
${ }^{1}$ http://gnu.org/licenses/agpl

2 http: //apache.org/licenses/

${ }^{3}$ Morin et al. 2012; http://opensource.org/licenses/bsd-license.php

${ }^{1}$ http://www.eclipse.org/legal/epl-v10.html
} 
Eomes eomesodermin homolog

$$
=\text { Tbr2 gene id: } 13813
$$

EPL Eclipse Public License ${ }^{1}$

ES embryonic stem cells

$\mathrm{EtBr}$ ethidium bromide

FACS fluorescence-activated cell sorting

ferricyanide hexacyanoferrate(III)

$$
=\left[\mathrm{Fe}(\mathrm{CN})_{6}\right]^{3-}
$$

ferrocyanide hexacyanoferrate(II) $=\left[\mathrm{Fe}(\mathrm{CN})_{6}\right]^{4-}$

Fezf2 Fez family zinc finger $2=\mathrm{Fezl}$ = Zfp312 gene id: 54713

FGF fibroblast growth factor (gene/protein family)

Fgf8 fibroblast growth factor 8 gene id: 14179

fig figure (also figures)

Fnp7 non-phosphorylated medium neurofilament (monoclonal antibody)

Foxp2 forkhead box P2 gene id: 114142

fw forward

GABA g-aminobutyric acid

Gap43 growth associated protein $43=B-50$ = Basp2 gene id: 14432

GE ganglionic eminence

Gfap glial fibrillary acidic protein gene id: 14580

GFP green fluorescent protein

GPL GNU General Public License ${ }^{2}$

h hour (also hours)

$\mathrm{Hb}$ habenula

HBSS Hank's balanced salt solution

HC hippocampal commissure

HE hematoxylin eosin

HF hippocampal fimbria and fornix

Hip hippocampus

HLH helix-loop-helix

HRP horseradish peroxidase

IC internal capsule

id referencing previously cited source (idem, latin)

IF immunofluorescence

IHC immunohistochemistry

IRES internal ribosomal entry site

ISH in-situ hybridization

IZ intermediate zone

kbp kilo base pair (also kilo base pairs)

I lateral

L1cam L1 cell adhesion molecule gene id: 16728

lacZ E. coli b-galactosidase (part of E. coli lac operon)

LGE lateral ganglionic eminence

LGPL GNU library general public license ${ }^{3}$

Lis1 lissencephaly $1=$ Pafah $1 b 1$ gene id: 18472

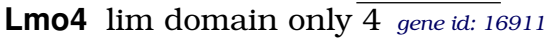

LOT lateral olfactory tract
LPPL LaTeX project public license ${ }^{4}$

LV lateral ventricle

M marker

m medial

Map2 microtubule associated protein 2 = Mtap2 gene id: 17756

Mapt microtubule associated protein $\mathrm{t}$ $=$ Tau gene id: 17762

Mb midbrain

Mbp myelin basic protein gene id: 17196

MGE medial ganglionic eminence

mGFP membrane targeted GFP

MGI mouse genome informatics database ${ }^{5}$

MHB midbrain-hindbrain boundary

min minute (also minutes)

MPL Mozilla public license ${ }^{6}$

mRNA messenger RNA

MZ marginal zone

NBF neutrally buffered formalin ( $4 \%$ in PBS)

Nefm medium neurofilament $=N F 160$ $=N F-M$ gene id: 18040

NeuN neuronal nuclear antigen $=$ Rbfox3 $=$ Fox -3 gene id: 52897

Neurod1 neuronal differentiation factor 1 $=$ NeuroD $=$ Beta $2=$ BHF -1

$=$ bHLHa3 gene id: 18012

Neurod2 neuronal differentiation factor 2 $=N D R F=K W 8=b H L H a 1$ gene id: 18013

Neurod4 neuronal differentiation factor 4 $=$ NeuroM $=$ Math3 $=$ bHLHa4 gene id: 11923

Neurod6 neuronal differentiation factor 6 $=$ NEX $=$ Math2 = bHLHa2 gene id: 11922

Neurog2 neurogenin $2=\operatorname{Ngn} 2=$ Atoh 4 $=$ Math4a $=b H L H a 8$ gene id: 11924

NGF nerve growth factor gene id: 18049

NMDA N-Methyl-D-aspartic acid (glutamate receptor agonist)

Nmdar1 ionotropic NMDA glutamate receptor, subunit $1=$ Grin 1 gene id: 14810

NP40 nonyl phenoxylpolyethoxylethanol

Nr2e1 Nuclear receptor family $2 \mathrm{e} 1=T l x$ $=$ tailless gene id: 21907

Nr2f1 Nuclear receptor family $2 \mathrm{f} 1=$ COUP-TF 1 (chicken ovalbumin upstream promoter transcription factor 1) gene id: 13865

NST nucleus of the solitary tract

OB olfactory bulb

ORF open reading frame

$\mathbf{P}$ postnatal day

p page (also pages)

Pax6 paired box gene $6=$ Sey = Dey gene id: 18508

PB Probst bundle

PBS phosphate buffered saline

\footnotetext{
${ }^{2}$ Morin et al. 2012); http://gnu.org/licenses/gpl

$3_{\text {http://gnu.org/licenses/lgpl }}$

${ }^{4}$ http://latex-project.org/lppl/

${ }^{5}$ Blake et al. 2014; http://informatics.jax.org/mgihome/nomen/

$6_{\text {http: //mozilla.org/MPL }}$
} 
Pcna proliferating cell nuclear antigen gene id: 18538

PCR polymerase chain reaction

PD public domain

PF posterior funiculus

PFA paraformaldehyde

PI3K phosphatidylinositol 3-kinase

PN pontine nucleus

PP preplate

PSPB pallium/subpallium boundary (antihem)

PVN periventricular nucleus of the hypothalamus

QPL 3 public license ${ }^{1}$

qRT-PCR quantitative reverse transcriptase PCR

RNA ribonucleic acid

Robo1 roundabout 1 gene id: 19876

rpm rotations per minute

RT room temperature

RT-PCR reverse transcriptase PCR

rv reverse

Satb2 special AT-rich sequence binding protein 2 gene id: 212712

SC spinal cord

sect section (also sections)

SEM standard error of the mean

Sema6a Semaphorin $6 a=$ Semaq gene id: 20358 sEPSC spontaneous excitatory postsynaptic current

Shh sonic hedgehog $=H x=H x l 3$ gene id: 20423

SNP single nucleotide polymorphism

Sox5 SRY-box 5 gene id: 20678

SP subplate

Sp8 trans-acting transcription factor 8 gene id: 320145

ST stria terminalis

Str striatum
SuM supramammillary nucleus

SVZ subventricular zone

T4 thyroxine

tab table

Tbr1 T (Brachyury) brain 1 gene id: 21375

TCA thalamocortical afferent axons

Tcf3 transcription factor $3=M E 2=E 2 A$ $=E 12 / 47$ gene id: 21423

Tcf4 transcription factor $4=M E 2=E 2.2$ $=$ ITF 2 gene id: 21413

TCT thalamocortical tract

TE tris EDTA

Tg tansgene (also transgenic)

Th thalamus

TKO triple-deficient mice ('triple knockout')

TM melting temperature

TRH thyreotropin releasing hormone

TSH thyroid-stimulating hormone

Tuj1 class III $\beta$-tubulin $=\underline{\text { Tubb3 }}$ gene id: 22152 (monoclonal antibody)

TUNEL terminal deoxynucleotidyl transferase dUTP nick end labeling (apoptose assay)

UTR untranslated region

UV ultra violet (light)

v ventral

Vglut1 vesicular glutamate transporter 1 $=\underline{\text { Slc17a7 }}$ gene id: 72961

VIdIr very low density lipoprotein receptor gene id: 22359

vs compared with (versus, latin)

VZ ventricular zone

WNT Wingless and Int homolog (gene/protein family)

Wt wild type

X-gal 5-bromo-2-chloro-3-indoyl- $\beta$-Dgalactopyranoside $(\beta$-galactosidase substrate)

\footnotetext{
${ }_{1}$ http://caml.inria.fr/pub/old_caml_site/license/QPL.txt
} 


\section{List of Figures}

1 Basic histology of the mouse cerebral cortex . . . . . . . . . 5

2 Axis specification in neuronal tube and cerebral cortex . . . . . . . 7

3 Transcriptional control of neocortical arealization . . . . . . . . . 9

4 Major neuronal projection tracts of the cerebral cortex . . . . . . . 13

5 Structure and heterodimerization of bHLH proteins . . . . . . . . 14

6 Evolutionary conservation of the Neurod 1 bHLH domain . . . . . . . 15

7 Overview of atonal related bHLH proteins in mice . . . . . . . . . . 17

8 Neurod 1 expression in the developing cerebral cortex . . . . . . . . . 18

9 Neurod2 and Neurod6 expression in the developing cortex . . . . . . 20

10 Used transgenic alleles for Neurod1, Neurod2 and Neurod6 . . . . . 21

11 The principle of Cre-LoxP based gene inactivation . . . . . . . . . . . 24

12 The principle of Cre-LoxP based gene activation . . . . . . . . . 25

13 Neocortical Neurod6 expression is confined to the SVZ and CP . . . 28

14 Neurod6 is mostly expressed by postmitotic pyramidal neurons . . . 29

15 Absence of Neurod2 transcripts in Neurod2-Null mice . . . . . . . 30

16 Phenotype of Neurod2-Null mice . . . . . . . . . . . . . . . 31

17 Hypoplastic anterior commissure in Neurod2-Null mice . . . . . . . 32

18 Brain anatomy of newborn Neurod2/6 double-deficient mice . . . . 34

19 Absence of AC and CC in Neurod2/6 double-deficient mice . . . . 35

20 CP formation in newborn Neurod2/6 double-deficient mice . . . . . 36

21 Marginal zone and subplate in Neurod2/6 double-deficient mice . . 38

22 Upper cortex layers in Neurod2/6 double-deficient mice . . . . . . . 40

23 Deeper cortex layers in Neurod2/6 double-deficient mice . . . . . . 42

24 Brain anatomy of adult Neurod2/6 double-deficient mice . . . . . . 43

25 Callosal axon growth in Neurod $2 / 6$ double-deficient mice . . . . . . 45

26 Loss of Contactin 2 in Neurod2/6 double-deficient mice . . . . . . . 48

27 Midline fusion in Neurod2/6 double-deficient mice . . . . . . . . . . 49

28 Ectopic Robol expression in Neurod2/6 double-deficient mice . . . 50

29 Cortical axonal projections in Neurod2/6 double-deficient mice . . . 51

30 Pyramidal neurons are functional in absence of Neurod2/6 . . . . 53

31 Neocortical arealization in Neurod2/6 double-deficient mice . . . . . 55

32 Neurod 1 upregulation in Neurod2/6 double-deficient mice . . . . . 56

33 Brain anatomy of newborn Neurod $1 / 2 / 6$ triple-deficient mice . . . . 58

34 Hippocampal hypoplasia in Neurod 1/2/6 triple-deficient mice . . . 59

35 Loss of hippocampal pyramidal neurons . . . . . . . . . . . . . 61

36 Cortex development in the absence of NeuroD genes . . . . . . . . . 62

37 Cortical plate formation in Neurod $1 / 2 / 6$ triple-deficient mice . . . . 63

38 Deeper layer identity in Neurod $1 / 2 / 6$ triple-deficient mice . . . . . . 64

39 Upper layer identity in Neurod $1 / 2 / 6$ triple-deficient mice . . . . . . 65

40 Apoptosis in Neurod $1 / 2 / 6$ triple-deficient mice . . . . . . . . . . . 66

41 Intracortical connectivity in Neurod $1 / 2 / 6$ triple-deficient mice . . . 68 
42 Subcortical connectivity in Neurod $1 / 2 / 6$ triple-deficient mice . . . . 69

43 Thalamocortical connectivity in the absence of Neurod1/2/6 . . . 71

44 Speculative model of genetic control in the developing neocortex . . 79

45 Major cortical fiber tracts in double- and triple-deficient mice . . . . 85

46 Neurod 1-Flox genotyping PCR . . . . . . . . . . . . . . . 97

47 Neurod2-Null genotyping PCR . . . . . . . . . . . . . . 98

48 Neurod6-Cre genotyping PCR . . . . . . . . . . . . . . . . 99

\section{List of Tables}

1 Formulation of tissue lysis reaction . . . . . . . . . . . . . . 94

2 Polymerase chain reaction . . . . . . . . . . . . . . 95

3 DNA gel electrophoresis . . . . . . . . . . . . . . . . 96

4 Primer sequences for qRT-PCR . . . . . . . . . . . . . . . 100

5 X-gal staining solution . . . . . . . . . . . . . . . . 103

6 Overview of primary antibodies . . . . . . . . . . . . 105

7 ISH In situ hybridization buffer . . . . . . . . . . . . . . 106 


\section{Index}

$129 / \mathrm{Sv}, 21,22, \mathbf{3 2}, 33,86$

Abbreviations, 129

Abstract, 1

Acknowledgments, 127

Acronyms, 129

Adult functions, 42, 52, 57, 86, 87, 89

Agarose gel, 94, 95

Agenesis

cornu ammonis, 60

corpus callosum, 1, 82

dentate gyrus, 21,58

Alignment

see sequence alignment

Alkaline phosphatase, 103

$\alpha$-helix, 14

Alzheimer's disease, 90

AMPA receptors, 22, 80

Amygdala, 19

Annealing temperature, 93

Anterior commissure, 13, 72

Antibodies (list), 104

Antihem, 7

ApE (software), 106

Apical progenitor cells, 10

Aplasia

hippocampus, 58, 84

see agenesis

Apnea, 33, 57

Apoptosis, 2, 21, 35, 59, 60, 65, $74,83,87$

Appendix, 126

Archicortex, 2, 8, 58

Arealization, 1, 7, 27, 40, 54, 72, 80

Asymmetric cell division, 9

Atonal, 17, 73

Auditory cortex, 8, 9

Autism, 4, 46

Avogadro (software), 106

Axis specification, 6, 6, 7

Axon

growth, 2, 11, 27, 44, 81

guidance, 1, 72

B27, 97

Background, 85 see genetic background

Barrel

see whisker-barrel

Basal progenitor cells, 10

Basic helix-loop-helix see bHLH

$\beta$-actin, 99

$$
\text { ISH probe, } 99
$$

$\beta$-cells, 18

$\beta$-galactosidase, 5, 29, 101

Beta2 (pancreas)

see Neurod1

Betz cells, 10

bHLH domain, 14

transcription factors, 1,14

class II, 15

neuronal, 17

Bhlhe22, 17

Bibliography, 108

Bicuculine, 98

Black reaction, 4

Blbp, 49

antibody, 104

Bone morphogenic proteins, 6

Brain

anatomy, 34, 57

development, 4

BrdU, 29

Breathing abnormalities, 33, 57

Breeding, 56

Brn2, 38, 40, 65

antibody, 104

Bromphenol blue, 95

C57BL/6, 21, 22, 32

Caenorhabditis elegans, 14

Cajal

Ramón y, 4

Retzius cells, 10, 37, 38, 79

Calb2, 38

antibody, 104

Callosal

agenesis, 1, 82

projections, 44

Camillo Golgi, 4

CANNTG (E-box), 15

cDNA, 99

Cell

culture, 96

cycle exit, 89

death

see apoptosis

Cellular recordings, 98

Cerebellum, 19

see granule neuron

Cerebral cortex

see cortex

Chemical synapse, 3,4

Cingulum, 1, 72

Citric buffer, 102

Classes of HLH proteins, 15

Closing Words, 90

ClustalX (software), 106

Cn3D (software), 106

Cnp, 43

antibody, 104

CNS anlage, 6, 7

Cntn 1

ISH probe, 99

Cntn2, 48, 50

antibody, 104, 128

ISH probe, 99

Cognitive impairment, 87

Commissural projections, 1

Compensation, 1, 24, 56

Conditional gene activation, 25

gene inactivation, 24, 26, 56

Connectivity, 1, 12, 66

cortical, 32, 44

intracortical, 66

subcortical, 68

thalamocortical, 69

Contents (table of), i

Contralateral projections, 13

Cooperative functions, 2

Cornu ammonis, 60

agenesis, 60

Corpus callosum, 13

agenesis, 1, 82

Cortex, 1

areas, 8

see arealization

cingulate, 1

development, 6, 34

layer, 13, 39, 40, 61

medial, 1

Cortical

antihem, 7

connectivity, $2,12,32,44,66$

hem, 7, 10

plate, 1,77

remodeling, 2

Corticofugal projections, 13

Corticospinal tract, $13,51,52$

Corticothalamic tract, 13

Cre

-LoxP system, 25

antibody, 104

ERT2, 88

Neurod6-Cre, 5, 36, 63

recombinase, $5,25,61,88$

reporter mice

axonal, 92

pMapt-mGFP/LacZ, 127

Rosa26, 92

Rosa26-LacZ, 127

Cryostat sectioning, 100

Ctgf, 11, 38, 39

Ctip2, 11, 41, 42, 64 antibody, 104

Cux 1, 11, 17

DAB, 103

Deeper layers, $10,18,37, \mathbf{4 1}, 41$, 55,86

Defasciculation, $1,45,46,82$

Delta/Notch System, 16

Dentate gyrus, 58

agenesis, 21, 58

Deparaffinization, 101, 102

Depression, 4, 89

Determination, 1, 27, 73

factor, 17,86

neuronal, 63

Developmental

cell death see apoptosis

Diet, 92 
Differentiation, 1, 2 factor, 17

Discussion, 72

DKO

see double-deficient see Neurod1/2 DKO mice see Neurod1/6 DKO mice DNA

primers, 93

program

see genetic program

Dorsoventral axis, 6

Double-deficient mice see Neurod1/2 DKO mice see Neurod1/6 DKO mice see Neurod2/6 DKO mice

Drosophila melanogaster, 14

E-box, 15, 74

E-proteins, 15

$\mathrm{E} 12, \mathrm{E} 2 \mathrm{~A}, \mathrm{E} 47$ see $T c f 3$

Ectoderm, 6

Ectopic

cells, 36-38, 40, 43, 62, 63

gene expression, 16

Neurod 1, 1, 28, 55, 56

Robo 1, 50

neurogenesis, 73

EGTA, 98

Electrophoresis, 94

Electrophysiology, 52, 98, 128

Enhancer of split, 16

Eomes, 11, 73, 76

Eph receptors, 12

Ephrins, 12

Epidermal growth factor, 7

Epileptic seizures, 21, 31, 33, 91

ES cells, 32

EUCOMM, 88

Evolution, 3, 14

Expression pattern, 1, 86

Neurod 1, 18

Neurod2/6, 19

Neurod4, 19

Eyeless, 16

Fasciculation, 1, 46, 82

Fiber tracts, 1, 3

Fibroblast growth factor, 7

Ficoll, 95

Figures (list), 132

Fixation, 100

Floor plate, 6, 7

Fnp7, 5, 43 antibody, 104

Foxp2, 11, 38, 41, 42, 64 antibody, 104

Functional redundancy, 23 see Redundancy

GABA receptors, 22

Gallyas

myelin staining, 32, 101

Ganglionic eminence, 7, 12

Gap43, 48, 53, 89

antibody, 104

ISH probe, 99

Gel electrophoresis, 94

Gene inactivation inducible, 88

Gene symbols, 129

Genetic

background, 21, 32, 33, 85, 86

code, 9

inactivation, 1, 20

modeling, 106

program, 3, 9, 27

Genotyping, 92

Gfap, 49

antibody, 104

GFP, 51, 68

membranal

see $\mathrm{MGFP}$

GIMP (software), 106

Glutamate

receptor, 53

transporter, 53

Gnumeric (software), 105

Golgi

Camillo, 4

staining, 4

Gradients, 6

Granule neurons, 58

cerebellum, 3, 18, 21, 22

differentiation, 58

hippocampus, 2, 21, 24

Graphviz (software), 106

Growth cone, 11

Handshake (thalamocortical), 69

HE staining, 34-36

Hem, 7, 10

HEPES, 98

Hes5, 16

Heterodimerization, 14, 15, 90, 91

Hippocampus, 8, 58, 72

aplasia, 58, 84

apoptosis, 65

commissure, 13

granule neurons, 21, 24, 58

mossy fiber pathway, 2

pyramidal neurons, 60

History (of neuroscience), 4

Homo sapiens, 14

Homodimerization, 15

Homolog, 15

Housing, 92

Hybridization buffer, 105

Hypothalamus, 19

Id proteins, 16

Identity, 1, 10, 64, 72

IHC

see Immunohistochemistry

Image processing, 106

ImageJ (software), 106

ImageMagick (software), 106

Immunofluorescence, 4

Immunohistochemistry, 102

In situ hybridization, 30, 103

buffer, 105

In utero electroporation, 98

Index, 134

Inducible Cre

see CreERT2

Inkscape (software), 107

Inside-out (cortex), 10

Insulin, 18

Interactivity (genetic), 3, 9

Intermediate progenitor cells, 10

zone, $1,10,38,44,56,76$

Interneurons, 6

Intestine, 18

Intracortical projections, 13, 66

Introduction, 3

Iontophoresis, 128

Ipsilateral projections, 13

ISH

see In situ hybridization

IZ, 56

Key regulator, 1

L1cam, 48, 67, 69 antibody, 104, 128

lacZ, 5, 22, 29, 67

Laminarization, 72 see Layering

${ }_{1} \mathrm{~T}_{\mathrm{E}} \mathrm{X}$ (software), 107

Layer

cortical, 13, 39, 40

Layering (cortex), 39, 41, 61

Learning, 46

Lethality, 21, 31, 33, 57, 85, 88

Lineage tracing, 26, 28

Linux, 105

List of

abbreviations, 129

antibodies, 104

figures, 132

references, 108

tables, 133

Lmo4, 38, 40, 54, 55, 65 antibody, 104, 128

Loading dye, 95

LoxP sites, 5, 25

LYX (software), 107

Manuscript, 107

Map2, 5, 36, 37, 43, 61, 63 antibody, 104

Mapt, 51, 87

Marginal zone, 10, 37, 38, 62

Master regulator, 16

Material, 92

Math2/3

see Neurod6/4

Mbp, 51

antibody, 104

ME2

see $T c f 3, T c f 4$

Medial cortex, 1

Memory, 89

Mendelian ratio, 33, 57

Mental retardation, 91

Methods, 92, 100

mGFP, 45, 51, 68

MGI gene symbols, 17, 129

Mice

breeding, 56

Cre reporters

pMapt-mGFP/LacZ, 127

Rosa26-LacZ, 127

Neurod 1

-Flox, 20, 55, 56, 127

-LacZ, 20

Neurod $1 / 2 / 6$ TKO, 1, 56

Neurod2

-LacZ, 22

-Null, 22, 31, 85, 127 
Neurod2/6 DKO, 1, 33

Neurod6

-Cre, 23, 23, 28, 127

-Null, 23, 23

transgenic, 4, 92

Microtome sectioning, 101

Midline, 1, 12, 72

crossing, 49

glia, 47

Migration, 2

radial, 9, 37, 61, 72

tangential, 10

Modeling (genetic), 106

Modifier gene, 86

Modularity (genetic), 3

Mossy fiber pathway, 2

Motor

cortex, 8,9

deficits, 91

Mouse lines, 92

mRNA-seqencing, 73

Multipolar

-to-bipolar transition, 37, 76

neurons, 11

Mus musculus, 14

Myc, 15

Myelin, 32

staining, 101

Myf5, 15, 16

MyoD, 15, 16

Myogenin, 15

\section{NBT/BCIP, 103}

NDRF

see Neurod 2

Neocortex, 2, 8, 58, 60, 72

Neocortical

connectivity, 44, 66

projections, 1

Netrins, 12

Network analysis, 3

NeuN, 5, 11, 36, 63 antibody, 104

Neural tube, 6, 7

Neurobasal medium, 97

NeuroD

family, 1, 15, 17, 27, 90

see Neurod1

Neurod 1, 1, 15, 17, 18, 73, 74

-Flox mice, 20, 56, 92, 127

-LacZ mice, 20

expression pattern, 86

inactivation, 20

ISH probe, 99

PCR, 94

upregulation, 55, 78

Neurod $1 / 2 / 6$ TKO, 56

Neurod $1 / 2 / 6$ TKO mice, 1

Neurod $1 / 6$ DKO, 24

Neurod2, 1, 19, 73

-LacZ mice, 22, 85

-Null mice, 22, 31, 33, 85, 85, 92,127

expression pattern, 86

inactivation, 22, 31

ISH probe, 99

PCR, 95

Neurod2/6 DKO, 1, 33

Neurod4, 17, 19, 73, 74

expression pattern, 87

Neurod6, 1, 19, 73
-Cre mice, 23, 28, 33, 92, 127

-CreERT2 mice, 88, 89

-Null mice, 23

expression pattern, 86

inactivation, 23, 24, 30

lineage, 5, 23, 28, 51, 56, 89

overexpression, $\mathbf{8 8}, 89$

PCR, 96

promoter activity, 1

Neuroectoderm, 6

Neuroepithelium, 9

Neurog2, 20, 73

Neurogenins, 15, 20, 73, 87

NeuroM, 19, 73

see Neurod4

Neuronal

bHLH proteins, 17

connectivity, 1, 44, 66

determination, 73, 73

differentiation, 89

groove, 6

identity, 1, 10

network, 3

plasticity, 88, 89

plate, 6

survival, 2, 72, 90

NEX

see Neurod6

NMDA receptors, 22, 53, 80

Nomenclature, 21, 129

NP40, 102

NTB/BCIP, 103

Ocaml (software), 106

Olfactory bulb, 8, 19

Oligs, 15, 73

ORF, 86

Outlook, 87

Over-migration, 38

Paleocortex, 8

Pancreas, 18

Paraffin sectioning, 101

Patch-clamp, 53

Pax6, 11, 16, 74, 82, 87

Pcna, 61 antibody, 104

PCR, 94

Neurod1-Flox, 94

Neurod2-Null, 95

Neurod6-Cre, 96

primers, 93

see $q R T-P C R$

Peek (expression), 19

Phylogenetic, 15

Pipette solution, 98

Pitt-Hopkins syndrome, 91

Plasticity related genes, 89

Plate

see cortical plate

PLL, 97

Polymerase chain reaction, 93 see PCR see $q R T$-PCR

Pontine nucleus, 19

Postnatal

lethality, $\mathbf{3 1}$

NeuroD functions, 2, 42, 52, 57, 86

Precursor cells, 18

Primary antibodies (list), 104

neuron culture, 96

Primer sequences, 99

Projections, 1, 44, 66

callosal, 44

subcortical, $\mathbf{5 0}$

Protein structure (bHLH), 14

Proteinase K, 93

Protomap, 39

PSPB, 7,10

Pyramidal neuron, 1, 75

determination, 73

differentiation, 60

identity, 10, 64

qRT-PCR, 99

$\mathrm{R}$ (software), 105

Radial

glia cells, 9, 37, 75

migration, 1, 9, 37, 61, 72, 79,

Ramón y Cajal, 4

Re-genotyping, 92

Recombinase

see cre-recombinase

Redundancy, 1, 23, 27, 56, 74, 88

Reelin, 10, 11, 37, 38

antibody, 104

Reference management, 107

References, 108

Regionalization see arealization

Respiratory insufficiency, 33

Results, 27

Retina, 71

Robo (Slit receptor), 12

Robo1, 49, 50, 67 antibody, 104, 128

Roof plate, 6, 7

RosaStopLacZ, 28

Rostrocaudal axis, 7

RT-PCR, 30, 99

Saccharomyces cerevisiae, 14

Satb2, 11, 38, 40-42, 65 antibody, 104, 128

Schizophrenia, 4

Scribus (software), 107

Sectioning

cryostat, 100

paraffin, 101

vibratome, 100

Seizures, 21, 31, 33, 91

Sema6a, 10

Semaphorins, 10, 12

sEPSC, 52, 53, 98

Sequence alignment, 15, 17, 106

Sequence similarity, 1

Signaling centers, 6,7

Simulation, 106

Single-deficient mice, 1

Slits (Robo receptors), 12

Small intestine, 18

SNP, 91

Software, 104

Somatosensory cortex, 2, 8, 9

Sonic hedgehog, 6

Sox5, 11, 38, 41, 42, 87

antibody, 104 
Specification, 2

Spinal cord, 6, 7, 71

StackReg (software), 106

Statistics, 105

Stem cells, 9, 73, 75

Striatum, 1, 7

afferents, 13, 51, 52

Subcerebral projections, 13

Subcortical projections, 13, 50, 68

Subplate, 12, 38, 39 38,76

Summary, 1

Survival

neuronal, 2, 28, 72, 90

rate, $21,33,57,85,88$

SVZ

see subventricular zone

Symmetric cell division, 9

Synaptic

function, 52

maturation, 2

Table of contents, $i$

Tables (list), 133

Tamoxifen, 88, 89

Tangential migration, 6, 10

Target gene, 89, 90

Tbr1, 36, 64, 76, 87 antibody, 104
ISH probe, 99

Tbr2, 76

see Eomes

Tcf3, 14, 15, 18, 74

Tcf4, 15, 16, 90, 91

Telencephalon, 7

Terminal differentiation, 2, 27, 64, 86,90

$\mathrm{T}_{\mathrm{E}} \mathrm{X}$ (software), 107

Thalamic projections, 1

afferents, 2

connectivity, 69

Thalamus, 69 afferents, 13, 51, 52

Thyroid hormone, 85

Thyroxine treatment, 85

Tissue

lysis, 93

preparation, 100

samples, 92

sectioning, 100

\section{TKO}

see Neurod1/2/6 TKO mice see triple-deficient

Topographic connections, 3

Transcription factor, 1, 3

Transgenic mice, 4, 47, 92

Triple-deficient mice see Neurod1/2/6 TKO mice
TurboReg (software), 106

Typesetting, 107

Unison (software), 105

Upper layers, 10, 39

Upregulation of Neurod1, 55

Ventricular

system, 6

zone, 9, 18, 28, 29, 37, 75, 89

Vglut 1, 53, 87

antibody, 104

Vibratome sectioning, 100

Visual cortex, 8, 9

Whisker-barrel cortex, 22, 27, 54, 80

Wingless homologs, 6

X-gal histochemistry, 5, 29, 101

Xylene, 101

cyanol, 95

Zone

see intermediate zone

see marginal zone

see subventricular zone

see ventricular zone

Zotero (software), 107 


\section{Zusammenfassung ${ }^{1}$}

The Brain is just the weight of God, For, heft them, pound for pound, And they will differ, if they do, As syllable from sound.

E. Dickison ${ }^{2}$

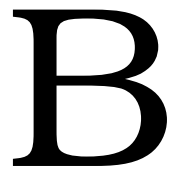

ASISCHE HELIX-Loop-Helix (bHLH)-Proteine bilden eine diverse Gruppe evolutionär gut konservierter Transkriptionsfaktoren. Viele transaktivierende bHLH-Proteine werden zelltyp- oder gewebespezifisch exprimiert und fungieren als wichtige Schlüsselregulatoren zellulärer Determinations- und Differenzierungsprozesse.

Die eng verwandten neuronalen bHLH-Gene Neurod1, Neurod2 und Neurod6 werden in differenzierenden Pyramidenneuronen des sich entwickelnden zerebralen Kortex exprimiert und stehen schon lange im Verdacht, deren Reifung zu steuern. In der Vergangenheit wurde jedes der drei Gene in Mäusen inaktiviert. Untersuchungen an den einfach-defizienten Tieren konnten jedoch keine wichtigen Funktionen in embryonalen Pyramidenneuronen identifizieren. Da die Aminosäuresequenzen und die Expressionsmuster der Faktoren sehr ähnlich sind, wurde angenommen, dass sie sich redundante Funktionalität teilen. Um dies zu überprüfen, habe ich Neurod2/6-doppel-defiziente Tiere gezüchtet und unter besonderer Berücksichtigung der Differenzierung von Pyramidenneuronen und der Konnektivität des zerebralen Kortex analysiert:

Die Experimente zeigen, dass Neurod2 und Neurod6 tatsächlich mehrere bisher unbekannte gemeinsame Funktionen haben, wobei jeder Faktor für den Verlust des jeweils anderen kompensieren kann. Zumindest eines der beiden Gene ist notwendig für (1) die Kontrolle der radialen Migration eines Teils der Pyramidenneurone, (2) die frühe Regionalisierung des zerebralen Kortex und (3) die Bildung kortikaler Projektionen vom Neokortex zum Striatum, zum Thalamus und zur kontralateralen Hemisphäre. Callosale Axone bilden in Neurod2/6-doppel-defizienten Mäusen Faserbündel die tangential in den medialen Kortex einwachsen, aber noch vor Erreichen des ipsilateralen Cingulums und vor dem Kontakt mit der Mittellinie stoppen und defaszikulieren. Es resultiert eine neue Variante der callosalen Agenesie, die nahelegt, dass es bisher nicht identifizierte Wachstumssignale im medialen Kortex gibt.

Die Expression von Neurod1, welche sich normalerweise auf die Subventrikularzone beschränkt, persistiert in radial migrierenden Pyramidenneuronen der

\footnotetext{
${ }^{1}$ Sinngemäße Übersetzung der englischsprachigen Zusammenfassung von Seite 1

${ }^{2}$ Dickinson 2013, no. CXXVI, verse 3
} 
Intermediärzone und der Kortikalplatte von Neurod2/6-doppel-defizienten Mäusen. Diese ektopische Neurod1-Expression kann dort den Verlust von Neurod2 und Neurod6 kompensieren. In einem weiteren Schritt habe ich konditionale Neurod1/2/6-tripel-defiziente Mäuse gezüchtet. In diesen Tieren wird das Neurod1-Gen durch selektive genetische Rekombination in all jenen Zellen, die über Neurod6-Promoteraktivität verfügen, irreversibel entfernt:

Wie erwartet, teilt sich Neurod 1 weitere gemeinsame Funktionen mit Neurod2 und Neurod6. Zumindest eines der drei Gene ist notwendig für die Differenzierung hippokampaler Pyramidenzellen und die Hemmung des programmierten Zelltods der unreifen Neuronen des Cornu Ammonis. Während die gemeinsame Inaktivierung von Neurod 1/2/6 zur Aplasie des Hippocampus führt, überlebt ein Großteil der neokortikalen Pyramidenzellen. Die terminale neuronale Differenzierung ist jedoch auch im Neokortex gestört und die neokortikale Konnektivität sehr stark reduziert.

Diese Arbeit zeigt, dass die Transkriptionsfaktoren der NeuroD-Familie gemeinsam die Differenzierung, das Überleben, die Migration und das axonale Wachstum von pyramidalen Neuronen des sich entwickelnden zerebralen Kortex steuern.

Während der Embryonalentwicklung ergeben sich folgende, teils überschneidende Funktionen der NeuroD-Gene: Die Differenzierung und das Überleben von hippocampalen Körnerzellen ist abhängig von Neurod $1 .{ }^{1}$ Die frühen Schritte der Differenzierung von hippocampalen Pyramidenneuronen und deren Überleben sind eine Funktion von wahlweise Neurod1, Neurod2 oder Neurod6. Spätere neuronale Differenzierungsschritte, die Regionalisierung des Neokortex und das gezielte Wachstum wichtiger neokortikaler Faserzüge basieren auf Funktionen von Neurod2 oder Neurod6, aber nicht von Neurod1. Der postnatale Umbau des somatosensorischen Kortex und die funktionale Integration thalamischer Afferenzen wurden bereits als strikt Neurod2-abhängig beschrieben. ${ }^{2}$

\footnotetext{
${ }^{1}$ Miyata et al. 1999; Liu et al. 2000

${ }^{2}$ Ince-Dunn et al. 2006; Molnár and Molnár 2006
} 